\title{
TAILORED TO SHRINK
}

A SUSTAINABLE METHOD OF CREATING 3D FORM UTILISING

NATURAL SHRINKAGE AND THE PRECISION OF DIGITAL DEPOSITION

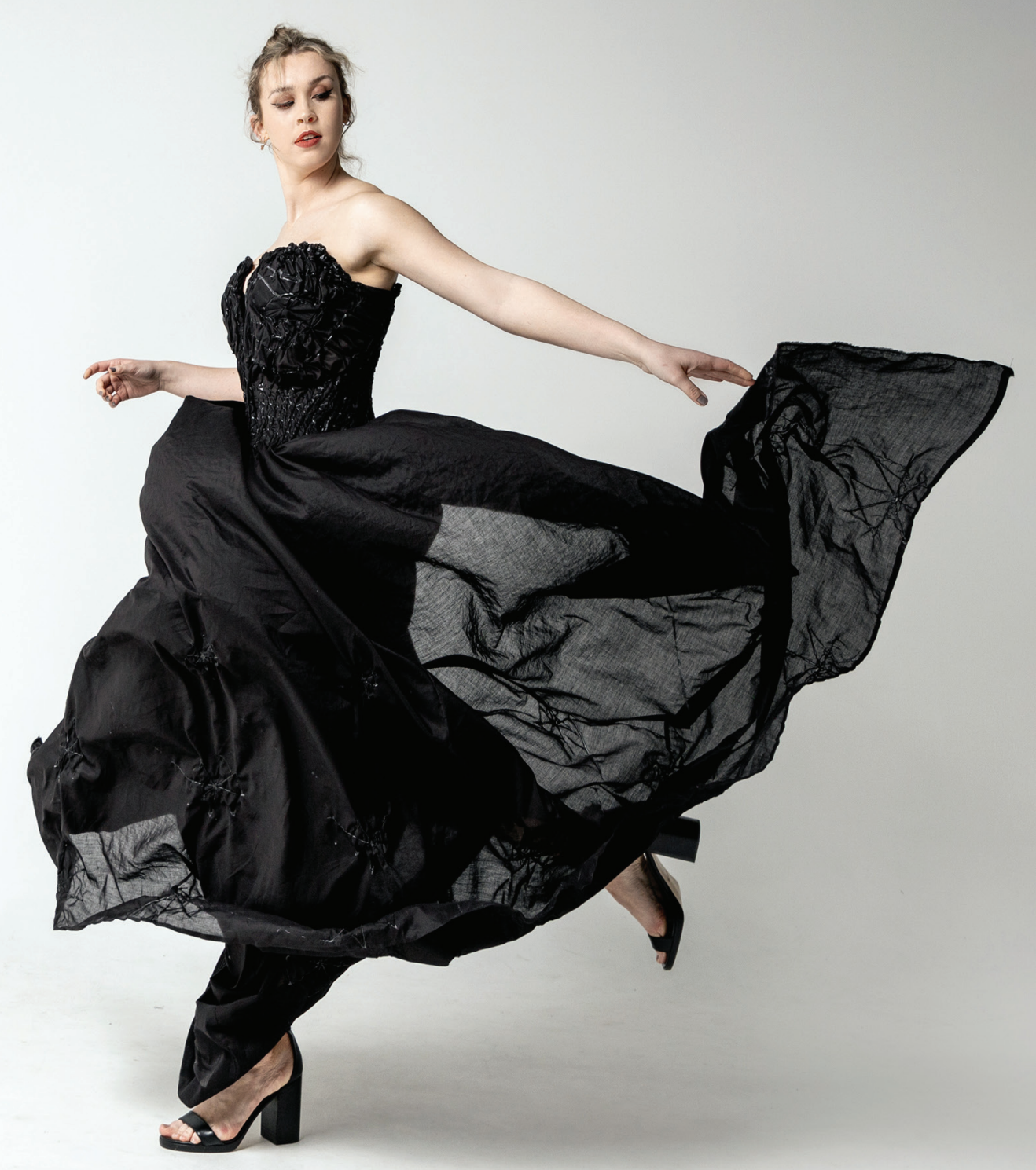




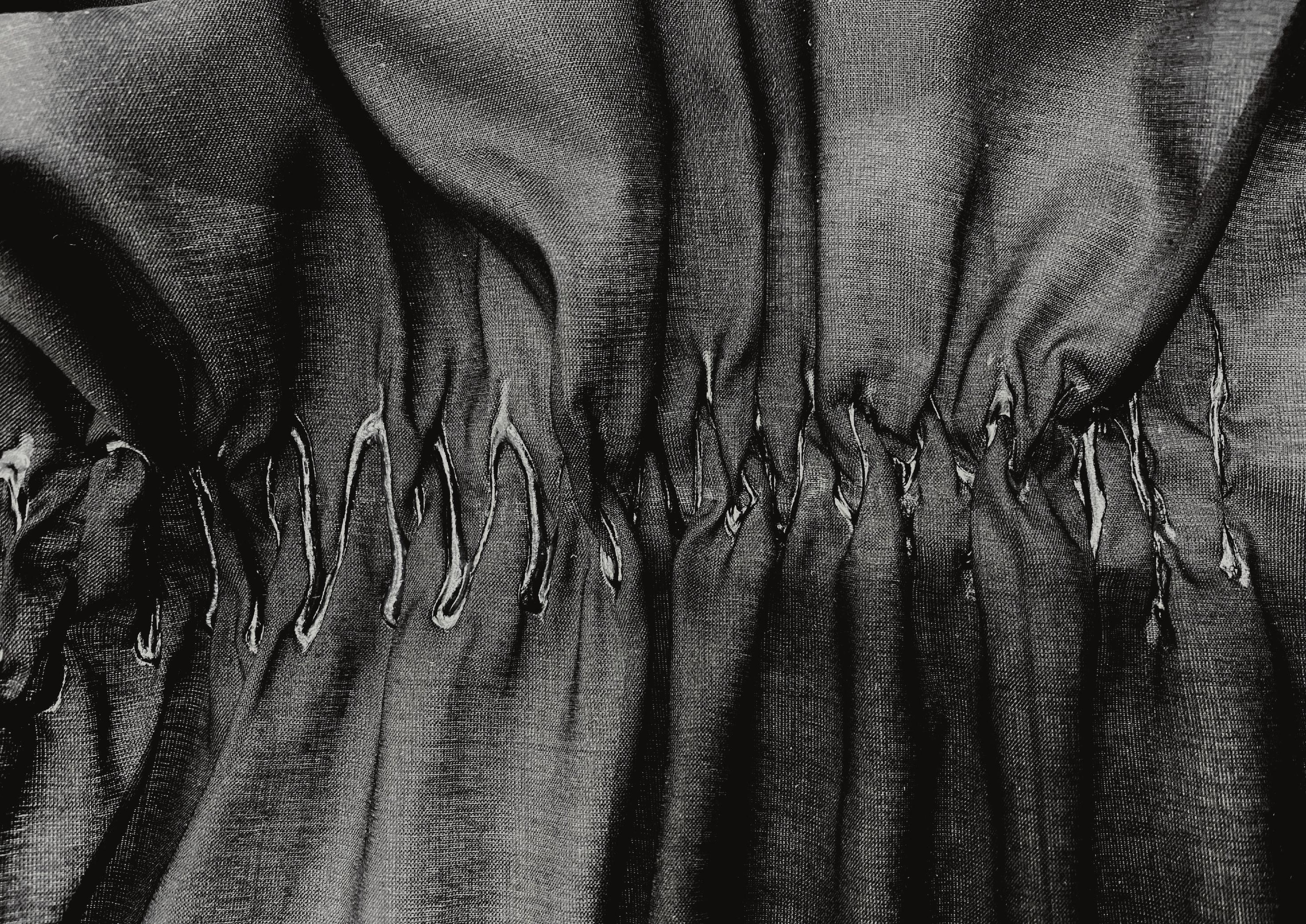


A ninety-point thesis submitted in fulfilments of the requirements for the degree of Master of Design Innovation.

Nayantara Sashikani Kuruppuarachchi

Victoria University of Wellington School of Design Innovation 


\section{ABSTRACT}

Many of the materials currently used within the fashion industry are petroleum-based synthetics. These are not sustainable and do not use environmentally friendly production processes. Interest in sustainably produced fabrics is increasing, but more radical approaches are also being explored, such as "growing" fabric and materials from mycelium. In haute couture fashion, the experimental use of 3D printing is being investigated using synthetic materials. However, there is an opportunity to combine the organic growth of natural materials with the precision of digital control within 3D printing.

In response to this opportunity, this research investigates the design and creation of haute couture 3D printed garments, using an eco-friendly, semi-liquid bio-composite material developed for syringe printing utilising the precision of a robotic arm. It adopts a cross-disciplinary approach to the field, oscillating between Industrial Design and Fashion Design with some experiments broadly based on science methodologies.

Prospective biomaterials were identified and an iterative process of testing combinations of suitable growing mediums, fabric substrates, and fungal cultures were explored to assess growth, material properties, and design opportunities. Mycelium from a native New Zealand fungus was selected and a series of 2D printed patterns were developed based on the Material Driven Design Method (MDD), to understand the relationship between digital placement, fabric substrate, and mycelium growth. Contraction of the flat fabric during the drying process allowed the authorship of undulating 3D fabric. Using a range of parametric software, strategies for 3D form creation to fit the body through controlled shrinkage were developed. A final series of 3D haute couture garments were co-created through the symbiotic relationship of digital control and natural processes.

Key words: parametric fashion, 4D printing, natural shrinkage, sustainable fashion, bio-based 3D printing 


\section{ACKNOWLEDGEMENTS}

Foremost, sincere gratitude to my supervisor Tim Miller, for all your guidance, patience and input throughout this project. You have always offerd encouragement and known when to push me further. Thank you for the great inspiration.

Further, the support and assistance by the VUW staff, especially by the workshop technician Hamish is immense.

Thank you Dr. Peter Buchanan and Landcare research for your guidance and support during early stages of the research, Jaco Pienaar from house of fungi for the valuable support, Paul Maka-kea and Emily for stunning photo shoot and Jean McDowall For proofreading at the crunch.

A huge thank to the MADE stream and National Science Challenge for their support and generous contribution., the Science for Technological Innovation Challenge, Portfolio 5 Materials, Manufacturing and Applications, Spearhead project: Additive manufacturing and 3D and/or 4D printing of bio-composites.

I am really grateful to Dinuka, Sara, and Vishwa, who were my constant sounding board for any and all my ideas. There is no way I would have been able to get to this point without you all.

Last but not the least, I would like to thank my parents and the family for the continuous support and patience throughout the entire study. 


\section{CONTENTS}

ABSTRACT

ACKNOWLEDGEMENTS

CONTENTS

III

LIST OF TABLES

GLOSSARY OF TERMS

CHAPTER I: LITERATURE REVIEW

BIO-COMPOSITES

BIO-COMPOSITES/BIO-BASED MATERIALS USED IN THE FASHION INDUSTRY

EXPERIMENTAL DESIGNS AND RESEARCH WITH MYCELIUM

PRECEDENCE DESIGN STUDY OF THE USE OF 3D PRINTED BIO-COMPOSITES

3D/4D PRINTING AS A PRODUCTION TECHNIQUE

HAUTE COUTURE FASHION

BIO-COUTURE, THE FUTURE

DIRECTION OF STUDY

CHAPTER 2: RESEARCH METHODOLOGY

INTRODUCTION

RESEARCH PROBLEM

METHODOLOGY

MATERIAL DRIVEN DESIGN (MDD) METHOD

CHAPTER 3: GROWING MATRIX AND SYRINGE PRINTING EXPERIMENTS 
COMMON PRACTICES IN GROWING MYCELIUM

EXPERIMENTS CONDUCTED USING THE MATERIAL DRIVEN DESIGN METHOD (MMD)

PHASE 1 - MYCELIUM GROWTH STUDY

PHASE 2 - SYRINGE PRINTING EXPERIMENTS AND BAKING

CHAPTER 4: DIGITAL/COMPUTER NUMERIC CONTROL (CNC) EXPERIMENTS 85

INTRODUCTION

WORKFLOW STRUCTURES FOR DIGITAL/CNC EXPERIMENTS

PAPM - POTATO AGAR PRINTABLE MEDIA EXPERIMENTS

MIPM - MYCELIUM INOCULATED PRINTABLE MEDIA

FURTHER PAPM EXPERIMENTS ON NATURAL COLOURS

EXPERIMENTS ON SHRINKAGE ANALYSIS

DESIGN IMPLICATIONS

CHAPTER 5: 4D PARAMETRIC TAILORING FOR SUSTAINABLE HAUTE COUTURE FASHION

INTRODUCTION

THE WORKFLOW OF BIO-BASED 4D PRINTING FOR HAUTE COUTURE FASHION

THE CONVENTIONAL WORKFLOW OF FASHION GARMENT PRODUCTION

THE DIGITAL WORKFLOW

FINAL DESIGNS

CHAPTER 6: DISCUSSION \& CONCLUSION

DISCUSSION

165

IMPLICATIONS \& APPLICATIONS

167

SUSTAINABILITY ASSURED

LIMITATIONS

FUTURE RESEARCH

CONCLUSION 


\section{LIST OF TABLES}

Table 1.1 Typical Smart Materials Used In 3D/4D Printing

Table 1.2 Properties Of 'Shape Polymers' From Qi's Experiment

Table 3.1 Observations With Respect To The Two Design Opportunity Parameters 


\section{GLOSSARY OF TERMS}

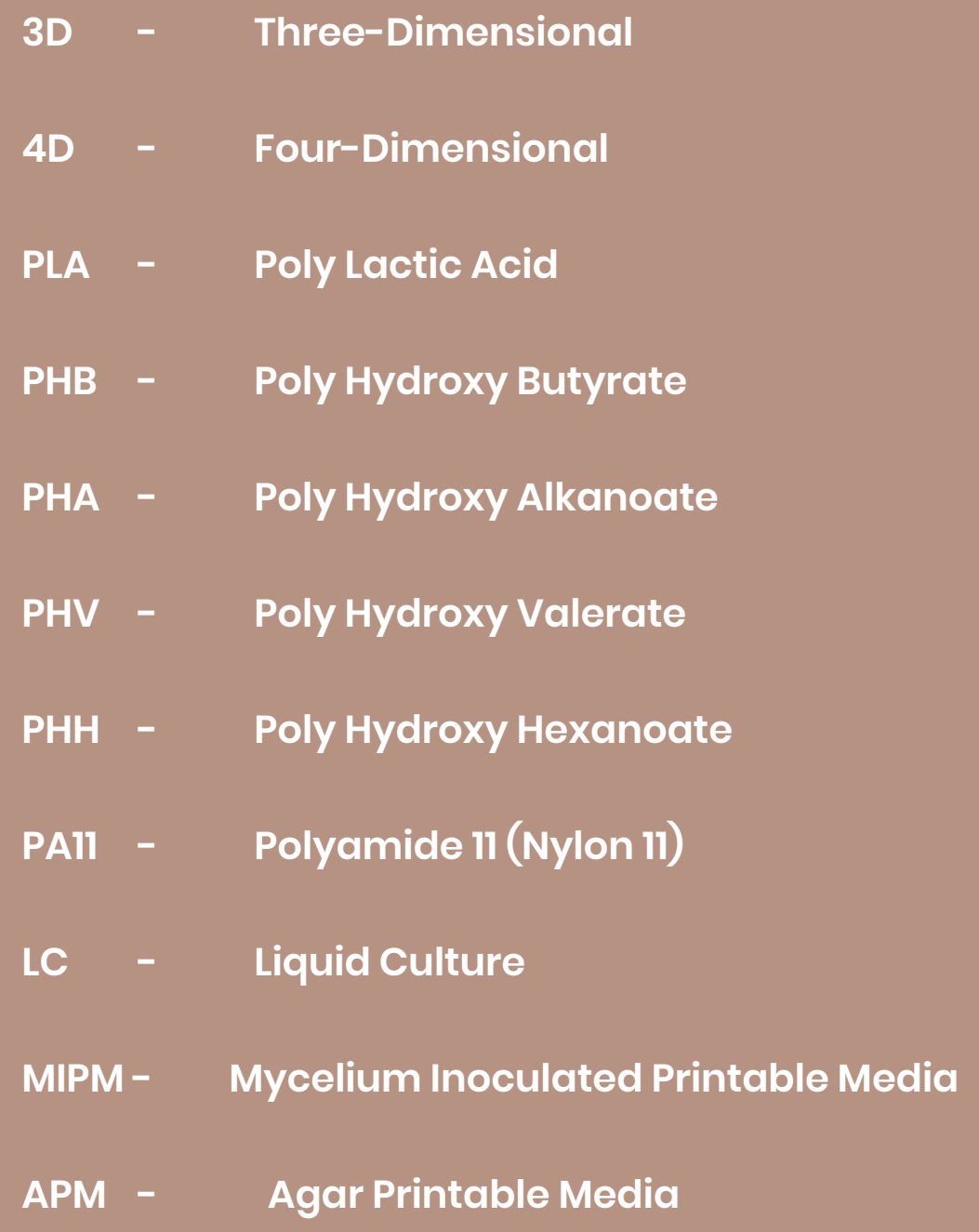




\section{LITERATURE REVIEW}

$\diamond$ BIO-COMPOSITES

$\diamond$ BIO-COMPOSITES/BIO-BASED MATERIALS

USED IN THE FASHION INDUSTRY

$\diamond$ EXPERIMENTAL DESIGNS AND RESEARCH

WITH MYCELIUM

$\diamond$ PRECEDENCE DESIGN STUDY OF THE USE

OF 3D PRINTED BIO-COMPOSITES

$\diamond$ 3D/4D PRINTING AS A PRODUCTION

TECHNIQUE

$\diamond$ HAUTE COUTURE FASHION

$\diamond$ BIO-COUTURE, THE FUTURE

$\diamond$ DIRECTION OF STUDY 


\section{LITERATURE REVIEW}

This section focuses on a review of the literature research into three main subsections: bio-composite materials, 3D/4D printing, and the use of 3D/4D printing in the fashion industry. Under each subsection definitions, applications, and their relevance to the present study are discussed, referencing the previous research and studies carried out.

Further, it is intended to build up an interrelationship among these primary subsections, to present the direction of this design research study exploration of laboratory-grown bio-composite material that could be 3D printed and used in the haute couture fashion industry. 


\section{BIO-COMPOSITES}

A composite material is developed by combining two or more materials with different chemical and physical properties, to achieve specialised characteristics such as lighter weight, improved strength, and stiffness. These desired characteristics of these composites are significantly changed compared to their base materials. (What is a composite material? A definitive guide, n.d.).

According to Sundakar (2012), composites are formed by combining a reinforcement matrix base and an additive resin. In bio-composites, natural fibres, usually derived from plants or cellulose, are used combined with matrix materials and additive materials.

Bio-composites can be classified in many ways. One popular method of classification is 'natural biomaterials' and 'synthetic biomaterials' (Khurshid, Najeeb, \& Zafar, 2019). Another classification (Jain, Borkar, \& Mantha, 2007) is based on the properties of fibrous material present in bio-composites.

Sundakar (2012) describes two ways that bio-composites could further be classified. One way would be based on the nature of the matrix used in the formation, such as polymer matrix, metal matrix, and ceramic matrix. The other way would be based on the properties of natural fibre used. The second classification implies that the source of natural fibre, too, plays a vital role in determining the performance of a bio-composite material, as it enables researchers to place more emphasis on applying them in suitable industries to achieve optimum benefits.

Another broad study on the sources of natural fibre to be used in the design and development of bio-composites has been reviewed by Yildizhan et al. (2018). According to Yildizhan et al. (2018) plant fibres, which are also called lignocellulosic fibres, are formed from three chemical substances known as cellulose, hemicelluloses, and lignin. They may further contain other different substances such as waxes, pectin, inorganics, starch, and protein depending on the plant, region, and species. Natural fibres are obtained from different components of plants, such as grass, wood, fruits, stems, leaves, and seeds. 
FIGURE 1.1

Classification of Bio-composites Based on Properties

of Fibrous Material
Within the context of bio-composites, the key focus in this research will be on the fungi-based bio-composites that could be used as a textile material. 


\section{BIO-COMPOSITES/BIO-BASED MATERIALS USED IN THE FASHION INDUSTRY}

With an understanding of the types, classifications, and sources of bio-composite materials, the study now focuses on describing synthetic and natural bio-composites, which are utilised in developing the latest trends in textile $\&$ fashion industry.

FIGURE 1.2

Poly Lactic Acid Fibre

Note: From Biomaterials in textiles: Poly-lactic acid fibres by Arnab Sen, 2015 (https://www.fibre2fashion.com/industryarticle/7574/biomaterials-in-textiles-poly-lactic-acid-fibres)

\section{POLY LACTIC ACID FIBRE}

In 1920, Wallace Carothers developed a bio-composite material called Poly Lactic Acid (PLA) (Figure 1.2) as an environment-friendly biodegradable polyester fibre (Sen, 2015), made out of corn.

Sen (2015) describes that because of the fast depletion of petroleum, a necessity has arisen to look for alternatives to replace the conventional textile fibre materials, which are made of petroleum-based products. As a result, researchers have been able to develop a bio-composite textile fibre using PLA, called Poly Lactic Acid Fibre. 


\section{BIOPLASTICS}

Another synthetic bio-composite is bioplastics.

Traditional plastics are developed from petroleum-based materials. When a plastic material consists of at least 20 percent or more renewable materials in its composition, it could be called a bioplastic (Cho, 2017). Thus, to reduce the harmfulness of traditional plastics, bioplastics are produced using renewable biomass sources such as vegetable fats and oils, corn starch, straw, woodchips, sawdust, and recycled food waste. According to Cho (2017), bioplastics are a significant improvement over traditional plastics in terms of the severity of their environmental impacts.

Types of bioplastics in use are listed below with their biobased material compositions. (Plainvanilla, 2021)

- Starch-Based Bioplastics - A simple bioplastic derived from corn starch. They are often mixed with biodegradable polyesters.

- Cellulose-Based Bioplastics - Produced using cellulose esters and cellulose derivatives.

- Protein-Based Bioplastics - Produced using protein sources such as wheat gluten, casein, and milk.

- Aliphatic Polyesters - A collection of biobased polyesters including PHB (polyhydroxybutyrate), PHA (polyhydroxyalkanoates), PHV (polyhydroxyvalerate), PHH (polyhydroxyhexanoate), PLA (polylacticacid) and PAll (polyamide 11), which are more or less sensitive to hydrolytic degradation and can be mixed with other compounds.

- Organic Polyethylene - Organic Polyethylene is produced from the fermentation of raw agricultural materials like sugar cane and corn, rather than fossil fuels.

Similar to synthetic bio-composites, natural bio-based materials sourced from substances like algae and fungi are also being widely experimented with by designers and researchers in the fashion and architecture industries. 


\section{ALGAE-BASED BIOMATERIALS}

Algae is a bacterium that plays the vital role of decomposing dead organisms and releasing their carbon back to the environment to balance the carbon cycle of the environment. (Nutrient Cycles, n.d.)

Similar bacteria which are sea-borne have been used to develop renewable algae-based biomaterials by an interdisciplinary research team, led by Dutch designer Eric Klarenbeek. In particular, they have researched the potentials of algae as an alternative for non-biodegradable plastics.

The team has managed to develop a polymer from algae that can be used to grow and create consumables and innovative products, illustrated in Figure 1.3, with the use of $3 \mathrm{D}$ printing technology (Reisky et al., 2019).

\section{FIGURE 1.3}

3D Printed Objects Using Dried Algae-Based Biomaterials by Eric

Klarenbeek and Maartje Dros

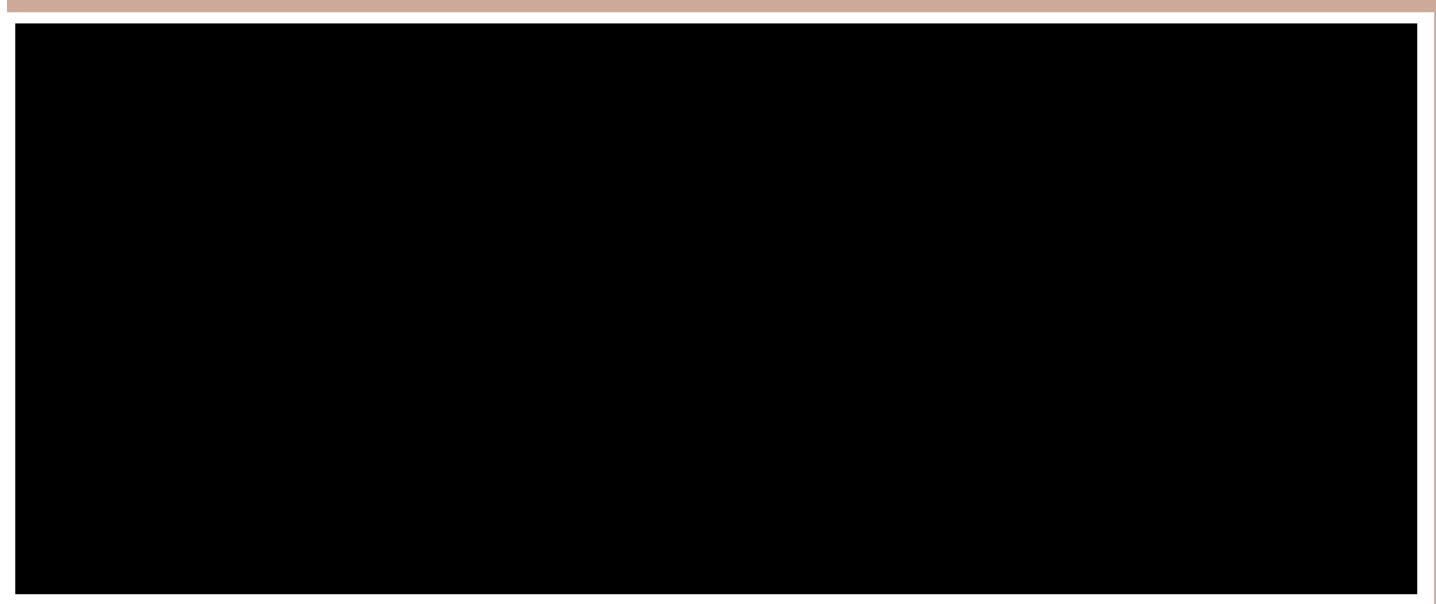

Note: From DESIGNERS OF THE VNVSVAL (https://www.ericklarenbeek.com/)

The designers have not just limited researching the potentials to algae plastics, but have also explored the possibility of using other organic matter to create alternative plastic biopolymers, including using cocoa bean shells, mycelium, and potato starch (Morris, 2017).

Another biomaterial source is fungi. This has long been explored as a naturally occurring material and has successfully been used in the food and medical industries and more recently as a bio-material alternative for building material in architecture and design applications.

Of particular interest is mycelium, which is the vegetative part of a fungus consisting of a mass of branching, thread-like hyphae, which naturally grows at a very rapid rate. (Karana et al., 2018). 


\section{MYCELIUM}

The root system of a mushroom growth is called mycelium, which consists of the growing 'stem' cells of the fungus. They grow by releasing enzymes from the hyphal tips of the mycelium to digest the surroundings and then absorb the nutrients. The cells will eventually branch and continue to branch as it grows, to build a vast, filamentous mycelial network. (Crosby, 2020).

Mycelium is identified as the largest living organism on earth. Haneef et al. (2017) describe the reason for mycelium's extensive growth as being its symbiotic relationship with the materials that feed it, forming entangled networks of branching fibres.

According to Collet (2017), mycelium can be cultivated on a range of cellulosic food such as fibres and particles, turning them into bio-composites. This evidence makes it clear that this biomaterial can easily be cultivated using natural resources. As mycelium grows naturally with the potential of being produced in quantity, it is a candidate for a more sustainable approach for the fashion industry. It will neither deplete the environment, nor will it have a negative environmental impact, and it is completely biodegradable.

FIGURE 1.4

Structure of Fungus

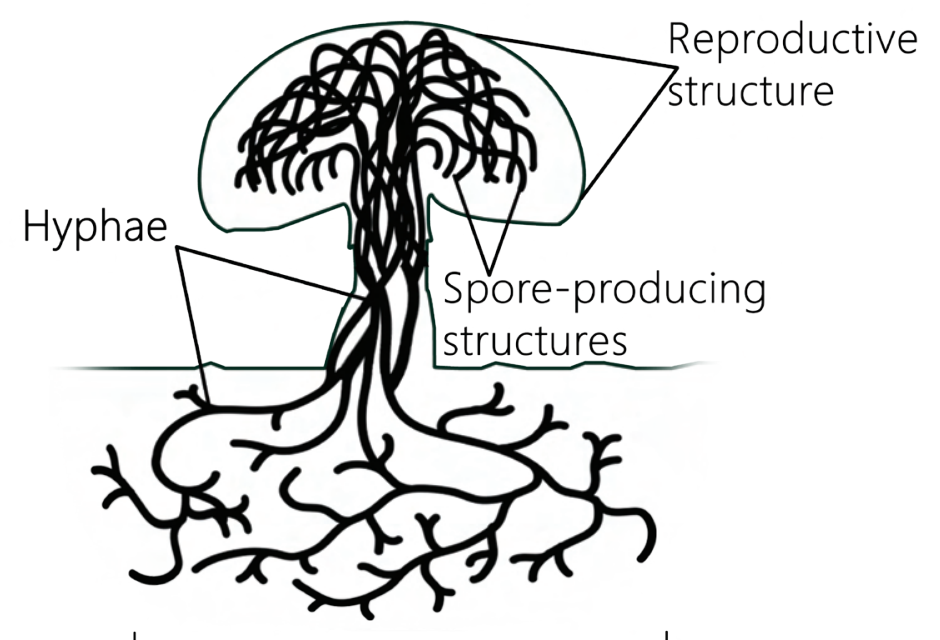

Figure 1.4 illustrates how mycelium in mushrooms grows underneath the supporting base, and presents a microscopic image of mycelium, with dimensions indicated in micrometres. 


\section{EXPERIMENTAL DESIGNS AND RESEARCH WITH MYCELIUM}

Because of the sustainable nature of mycelium, many researchers have investigated mycelium as a building material for architectural installations and design applications using mycelium-based composites.

FIGURE 1.5

'Hy-Fi' by The Living

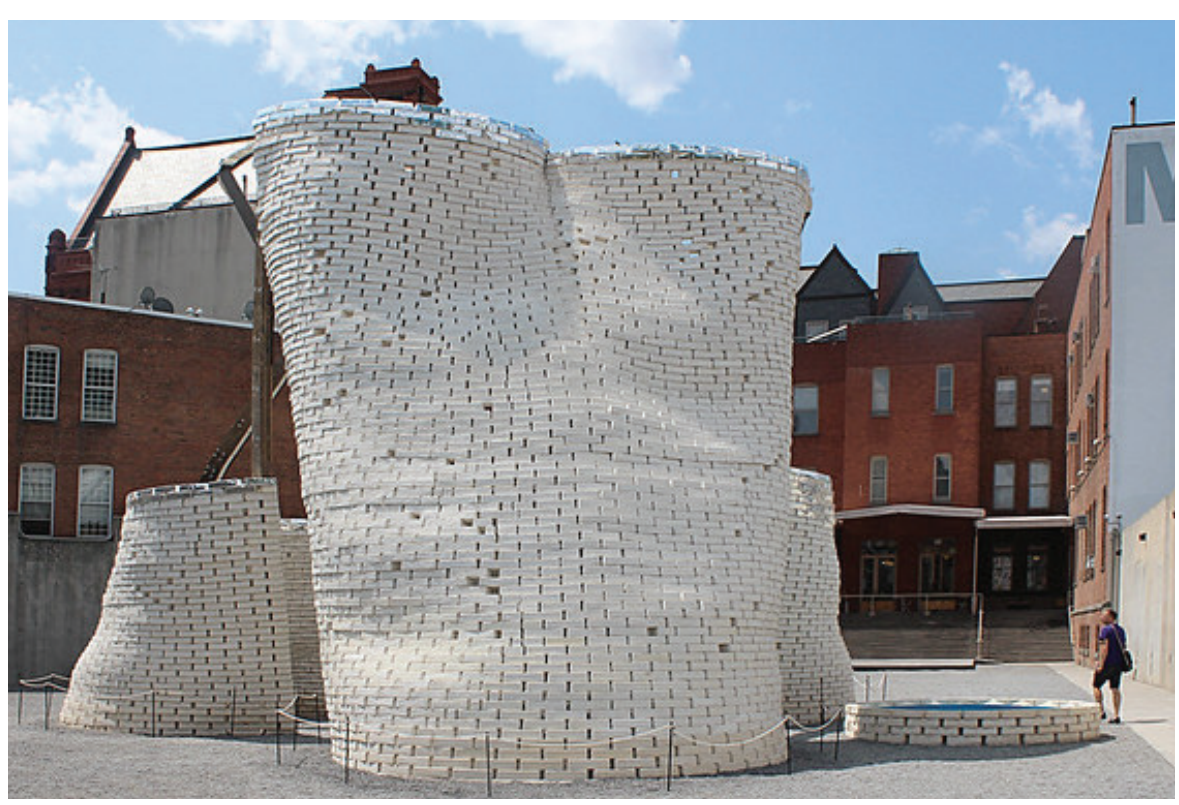

Note: From Hy-Fi, PSI YAP, New York City The Living, 2014 by trevor.patt (https://www.flickr.com/photos/trevorpatt/14886209656/in/photostream/)

\section{"HY-FI" BY THE LIVING - A ZERO CARBON EMISSIONS COMPOSTABLE STRUCTURE}

This is a cluster of circular towers over $12 \mathrm{~m}$ tall formed using 10,000 bricks that were naturally grown from shredded corn stalks and mushroom mycelium using recent advances in biotechnology combined with cutting-edge computation and engineering. (Lafarge, n.d.) Commissioned by the MoMA (Museum of Modern Arts) PSI Young Architects Program, these bricks required no energy and produced no waste or by-products. After three months of cultural events, the structure, as shown in Figure 1.5, was disassembled and the bricks have been decomposed to compost. 


\section{MYCOFLEX ${ }^{\mathrm{TM}}$}

Mycoflex ${ }^{\mathrm{TM}}$ is a sustainable foam made of $100 \%$ pure mycelium, which has superior properties over conventional petroleum-based materials.

Due to the inherent properties of this bio-based material, such as strength, heat resistance, insulation, resilience and its breathable nature, this bio-based product has been adopted for manufacturing sustainable components for packaging, skincare, apparel, food, and the leather industry.

\section{FIGURE 1.6}

Grown Products

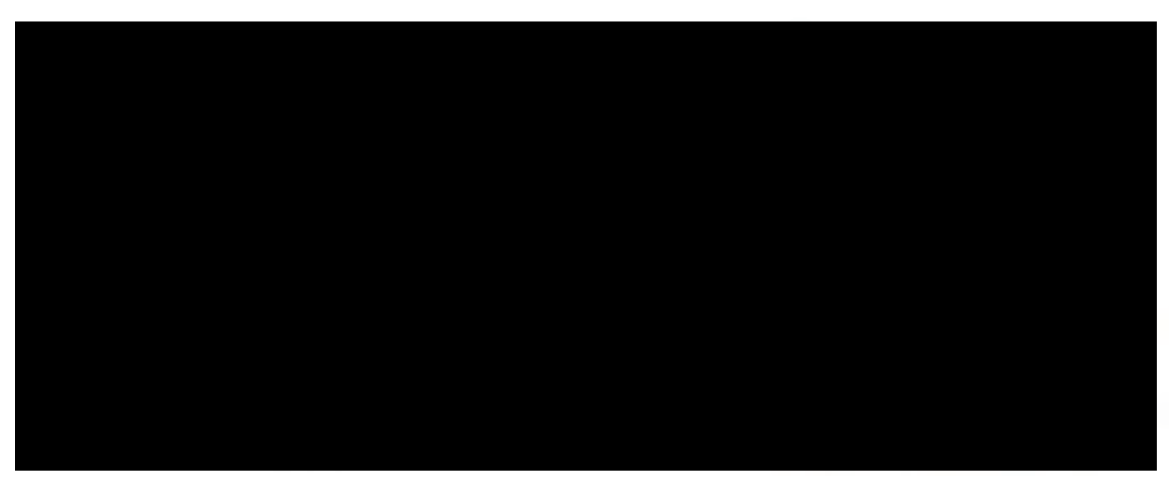

Note: From Interior \& Products by Grown.bio (https://www.grown.bio/)

\section{INTERIOR DESIGN AND PACKAGING PRODUCTS BY GROWN}

A Netherland-based company named Grown develops and sells a wide range of featured products made of mycelium-based biocomposites.

According to the manufacturer's website data ("Grown.bio", 2020), the special characteristics of Grown products (Figure 1.6) are listed as follows.

- Renewable raw materials. No oil-based sources, or products containing toxic elements

- Limitless availability, re-producible in Lab environment

- Flexible; it can be designed to any specification or desired shape

- Fire resistant

- VOC free (volatile organic compounds)

- Insulating, both temperature as well as sound

- Compostable, making the circle round 


\section{PRECEDENCE DESIGN STUDY OF THE USE OF 3D PRINTED BIO-COMPOSITES}

\section{THE AGUAHOJA COLLECTION BY NERI OXMAN}

Neri Oxman is a professor at Massachusetts Institute of Technology (MIT) and an expert in digital manufacturing. She has developed different innovations for art and architecture combining different disciplines of design, computing, biology, and material science. In many projects, Neri Oxmen uses 3D printing and fabrication technology to develop the designs. Most of her projects use animal fabrications or natural processed materials. She is an expert in material ecology and her experiments always address sustainability (MIT Media Lab People, 2020).

The Aguahoja collection as illustrated in Figure 1.7 and Figure 1.8 offers a material alternative to plastic, subverting the toxic waste cycle through the creation of biopolymer composites that exhibit tuneable properties with varied mechanical, optical, olfactory, and even gustatory properties. These renewable and biocompatible polymers leverage the power of natural resource cycles and can be materially 'programmed' to decay as they return to the earth, for the purposes of fuelling the new growth (Oxman, 2020).

FIGURE 1.7

Neri Oxman's Aguahoja Project

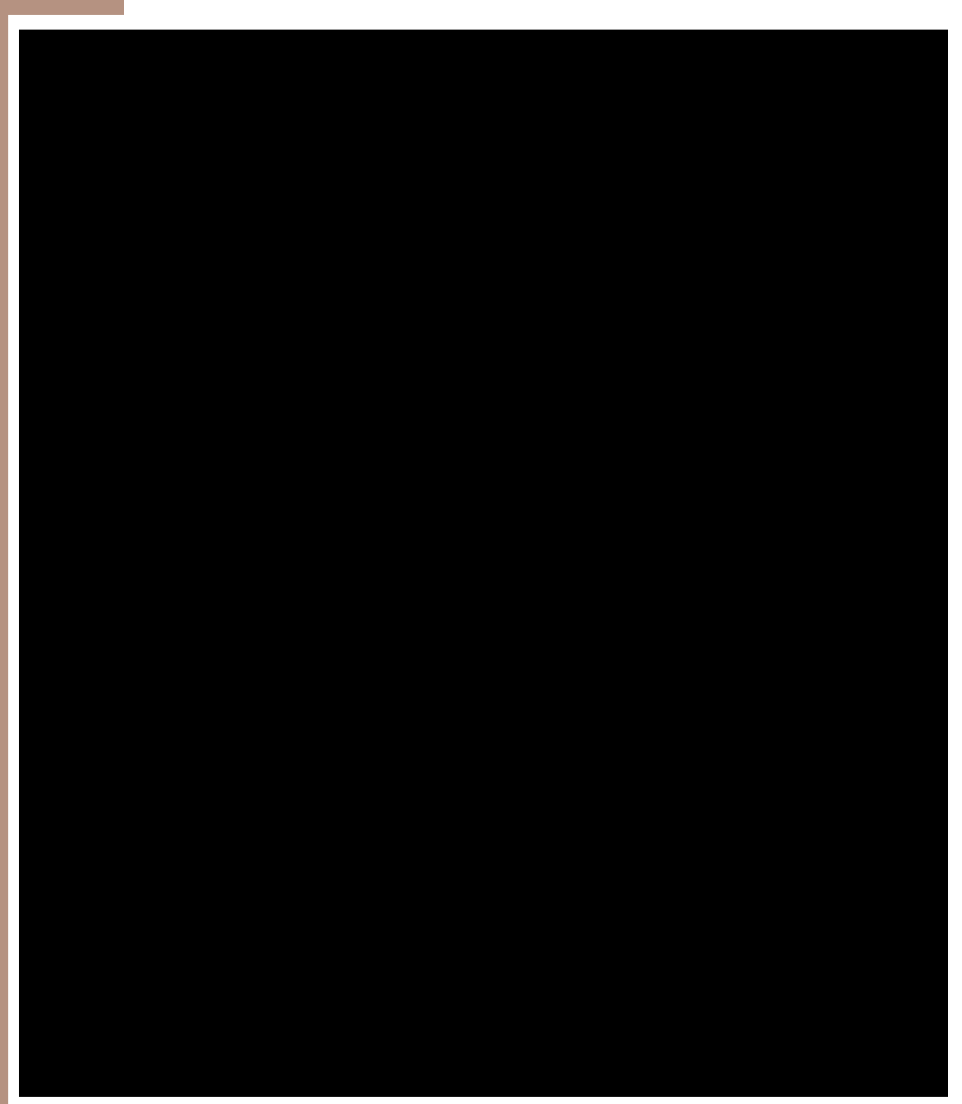

Note:From Biopolymer pavilion (https://oxman.com/projects/aguahoja) 


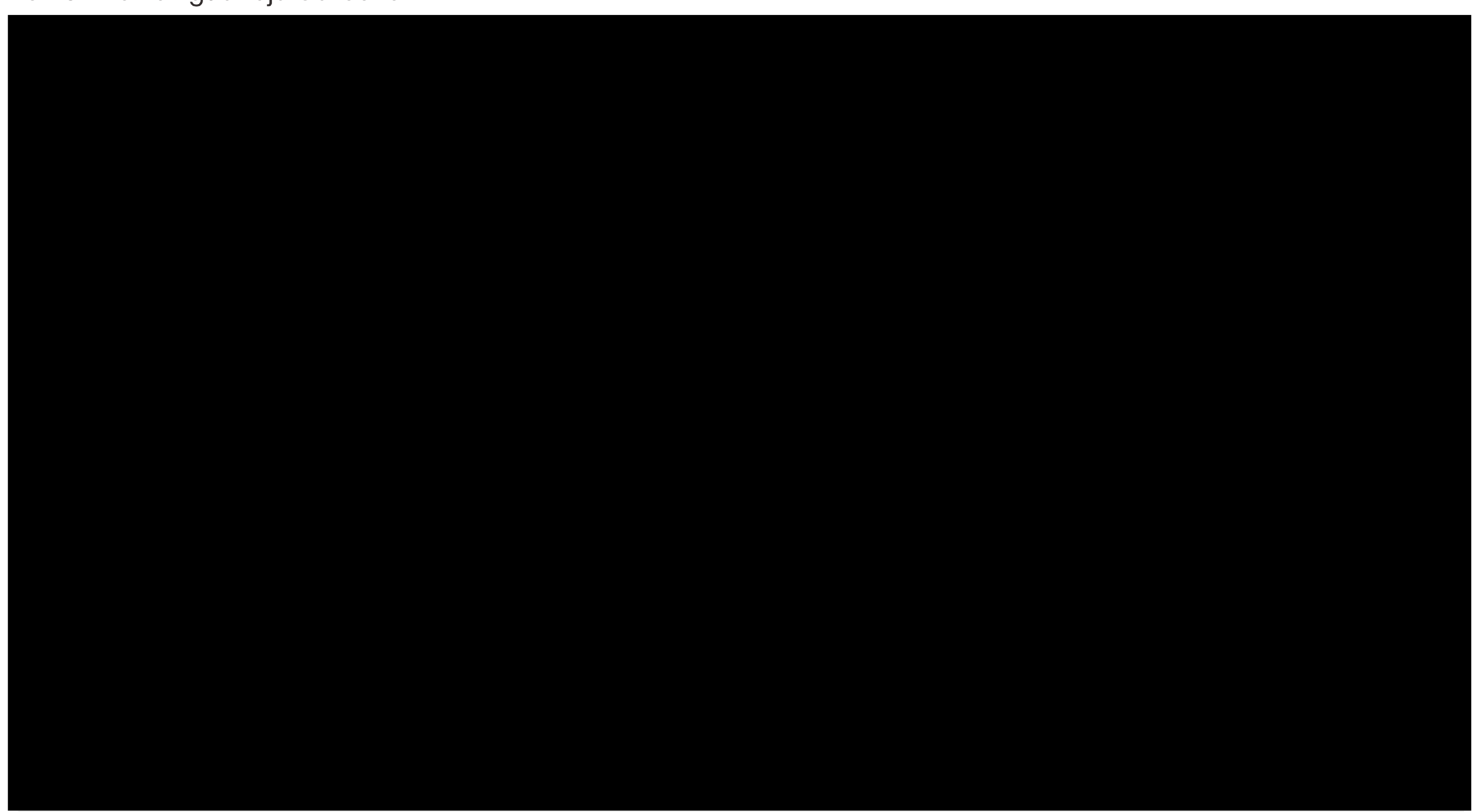

Note: From Parametric chemistry: a scientific design framework for 3D printing biocompatible multi-functional material combinations (https://oxman.com/projects/aguahoja) 


\section{MUSHROOM CHAIR BY ERIC KLARENBEEK}

This project was carried out by the artist Eric Klarenbeek (2014) from the Netherlands, using FDM printing techniques and ground straw-infused fungus as a filament for the printer. The adaptation he made is that the printer does not use heat. Mycelium acts as the glue by making the bio-composite (Krassenstein, 2014). The texture he achieved is similar to lightweight cork.

Further, it could be recognised that the low usage of heat and energy is a positive factor in the production of this biomaterial, further increasing its potential. The 'chair' (Figure 1.9) seems softer and lightweight, as straw has been used as the substrate. Additionally, mushrooms can be grown in the chair.

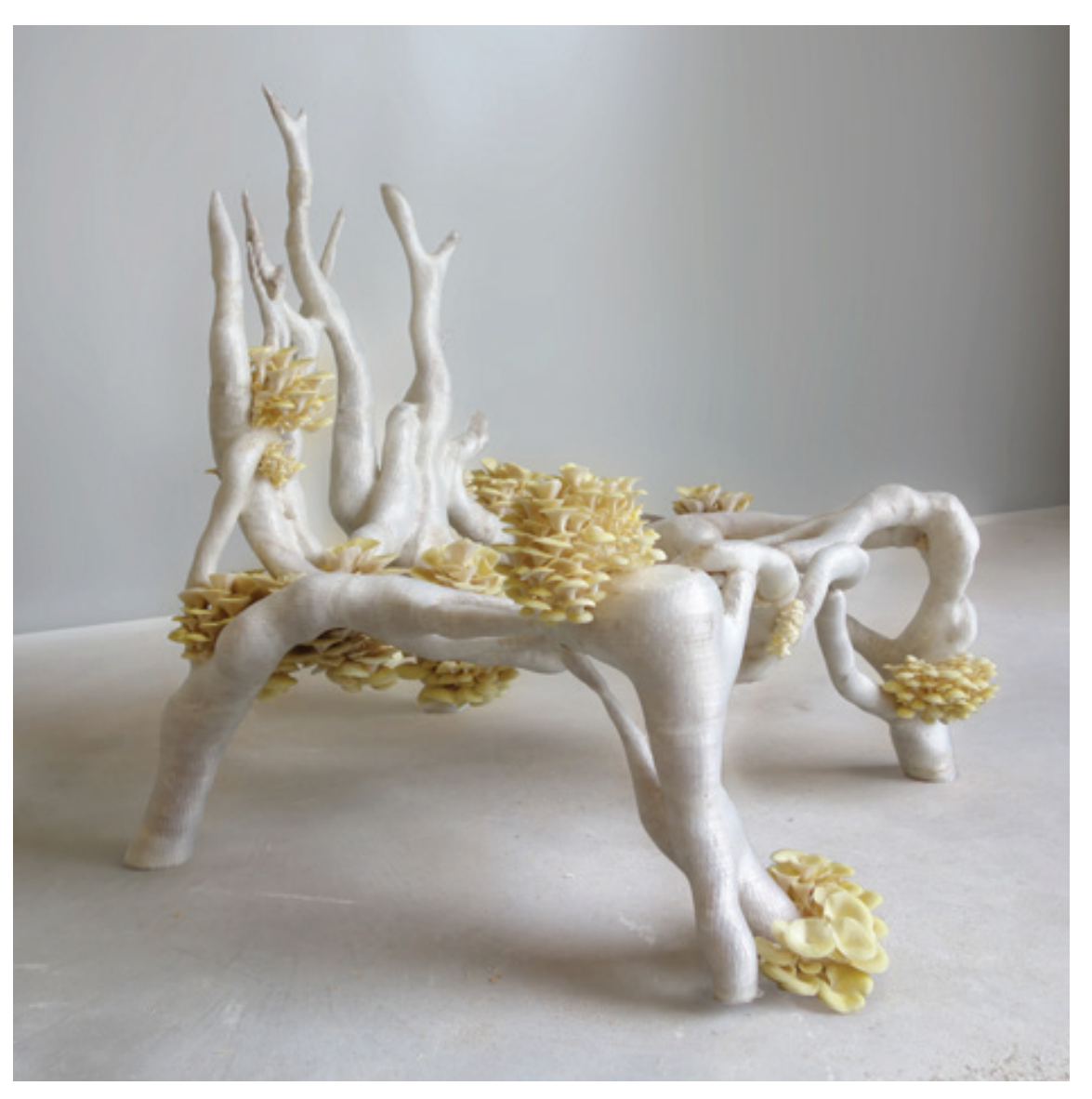

Note: From Mycelium Chair / Studio Klarenbeek \& Dros / www.dotunusual.com 


\section{GROWING MYCELIUM IN THE 3D PRINTED SCAFFOLDING BY ELIZE KOETSIER}

Koetsier (2020) conducted a study at Victoria University of Wellington that eliminated the use of synthetic materials, researching and designing more sustainable methods and manufacturing materials for temporary structures used at music festivals. The researcher developed a sustainable strategy for the 3D printing or digitally fabricated moulds using both natural and manmade techniques to combine them into a fabrication system. The study's main focus was on developing spatially printed structures by integrating mycelium-based composites to determine the form, materiality, and potential usage. The synergies of materials, methods of growth, and manipulation of mycelium-based composites were investigated. In addition, the study identified the suitability of mycelium growth for the creation of large-scale structures. The study found that the ability to use mycelium composites for spatial printing, form, and strength led to the establishment of alternative methods and distinctive design prospects to the conventional use of casting moulds. It presented an innovative manufacturing method of combining formwork and biomaterials, utilising the flexibility and precision of digital design and sustainable materials.
FIGURE 1.10

Experiments by Elize Koetsier, Growing Mycelium in 3D Printed Scaffolding

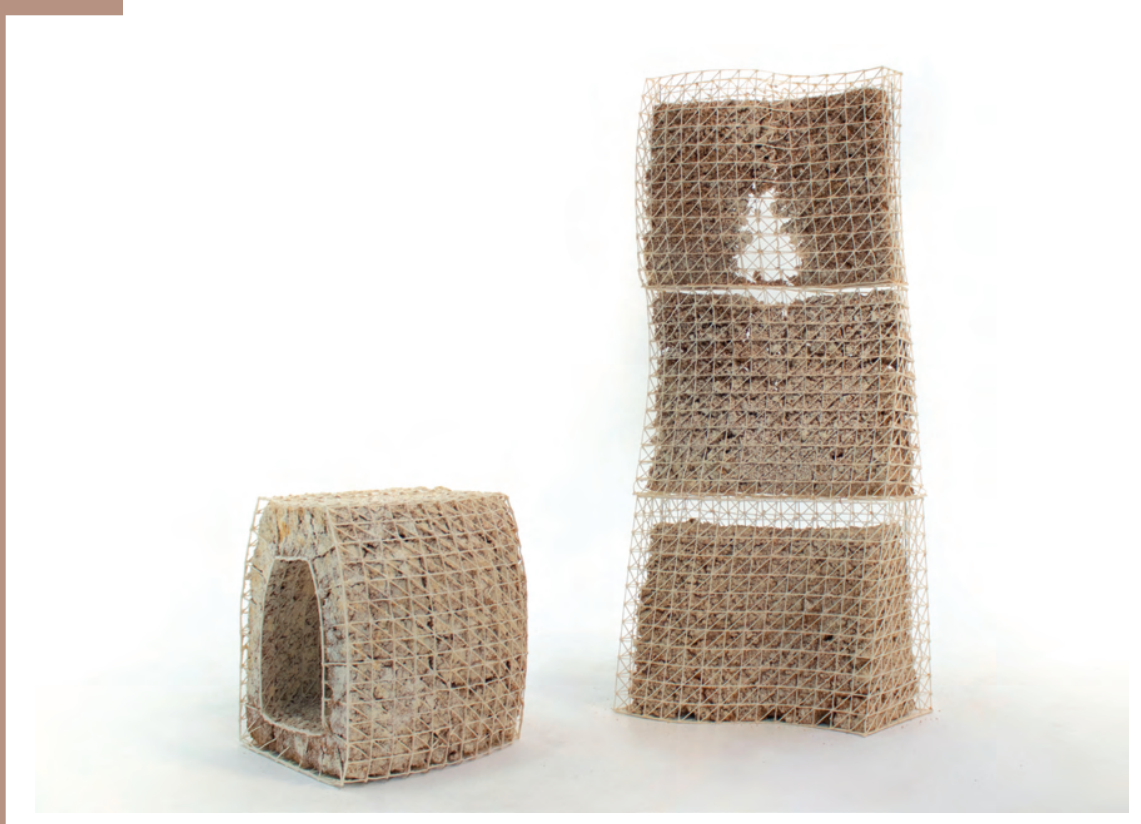

Note:From Designing with mycelium by Elize Koetsier, 2020

(https://openaccess.wgtn.ac.nz/articles/thesis/Designi ng_with_Mycelium_Utilising_spatial_printing_to_crea te_scaffolds_for_mycelium_growth/14152253) 


\section{SELF-PATTERNING MYCELIUM RUBBER}

Professor Carole Collet, Design \& Living Systems Lab, Central Saint Martins, University of the Arts, London identified ways to develop and utilise a unique characteristic of a living system like mycelium; its capacity for autopoiesis. Derived from Greek, the term autopoiesis refers to the capability of a system to maintain and reproduce itself by creating its parts and following up with the creation of other components.

FIGURE 1.11

Self-patterning Mycelium Rubber
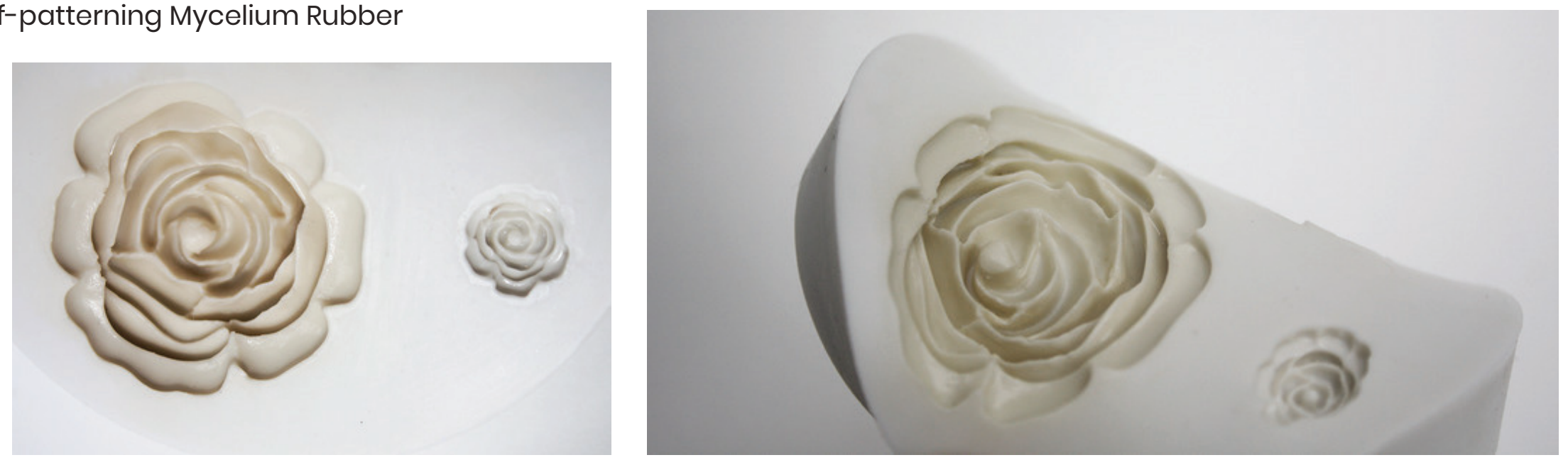

Note: From Self-patterned Mycelium Rubber and Mycelium Lace by Collet, Carole (2016) on UAL Research Online

(https://ualresearchonline.arts.ac.uk/id/eprint/10405/)
In one of the experiments, a protocol was developed that encouraged mycelium to manifest its self-organised behaviour in the form of visible patterns and exhibit the self-assembly qualities inherent in biological systems. (Collet, 2016)

The patterns shown in Figure 1.11 are formed by mycelium, stimulating its self-patterning abilities without being shaped by a mould.

This breakthrough discovery enabled researchers to experiment further on controlling the growth environment and observe the different patterns created by mycelium. 


\section{TARTAN MYCELIUM, MYCELIUM LACE AND MYCELIUM VELVET}

In another experimental design development conducted by Collet (2016), mycelium was allowed to be grown on substances like vintage lace, leaves or cloths, while providing a medium of waste coffee grounds as food. The shapes and patterns generated with the growth of mycelium over the base mediums shown in Figure 1.12 have been observed to be quite fascinating.

According to Collet (2016), research teams are conducting further experiments on enhancing the mycelium growth, to desired patterns as well as surface conditions.

FIGURE 1.12

Tartan Mycelium, Mycelium Lace and Mycelium Velvet

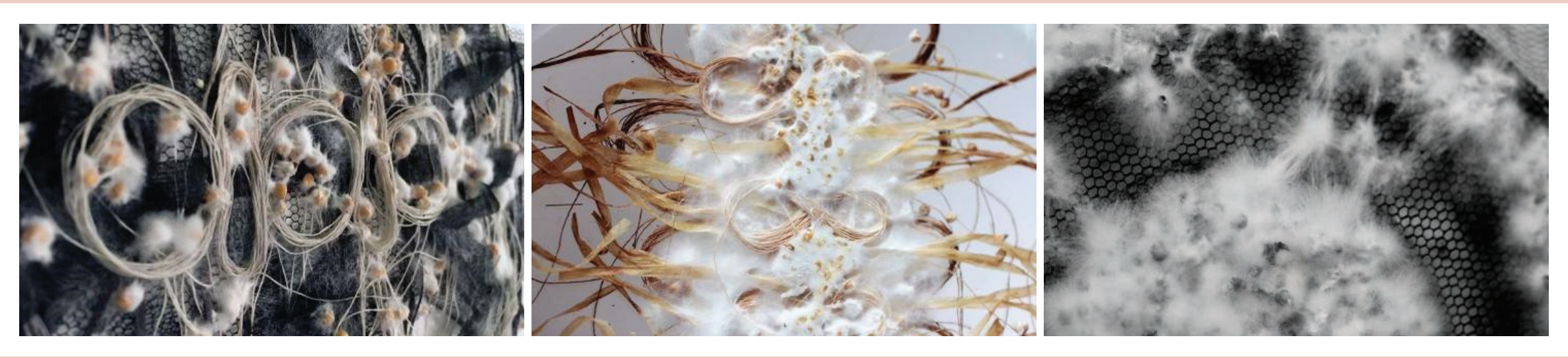

Note: From Mycelium Textiles by Collet, Carole (2019) on UAL Research Online (https://ualresearchonline.arts.ac.uk/id/eprint/15544/) 


\section{BLAST STUDIO}

The key concept of the blast studio is to develop a sustainable material by mixing urban waste with mycelium (Figure 1.13). Different objects resulted from these experiments are presented in Figure 1.14. They use a different technique as they directly 3D print with mycelium matrix.

\section{FIGURE 1.13}

\section{Key Concept of Blast Studio}

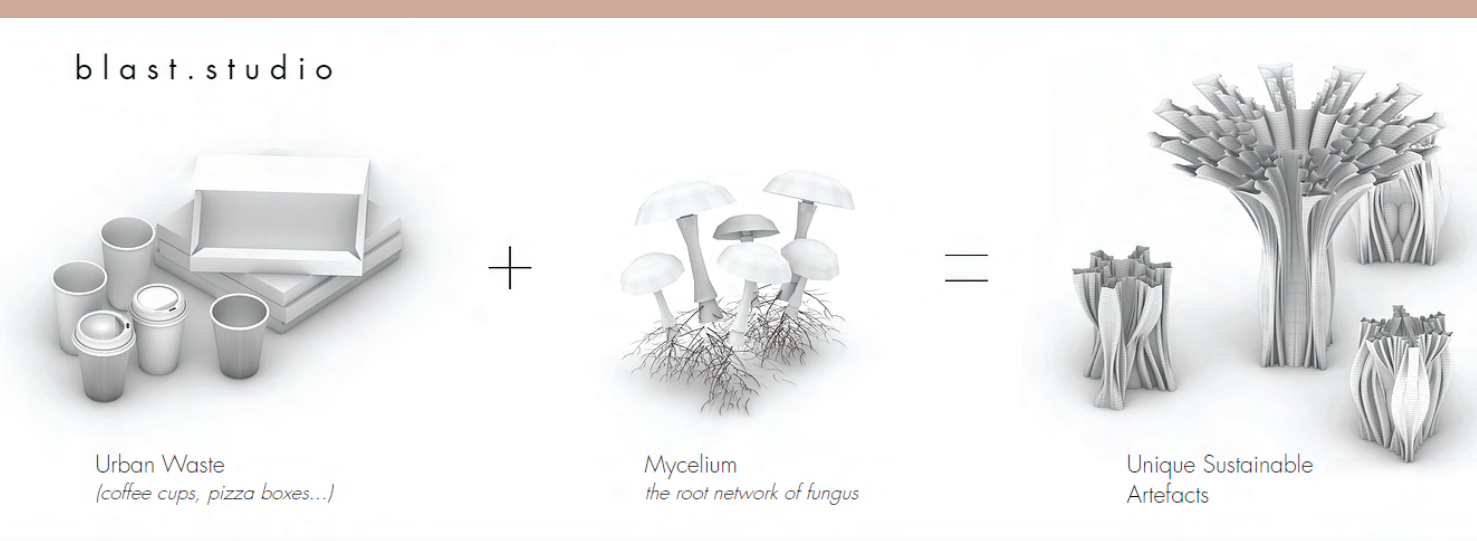

Note: From Objects from nature.Made with cardboard waste \& living organisms on

blast.studio (https://www.blast-studio.com/)

FIGURE 1.14

Different Objects of Blast Studio Experiments

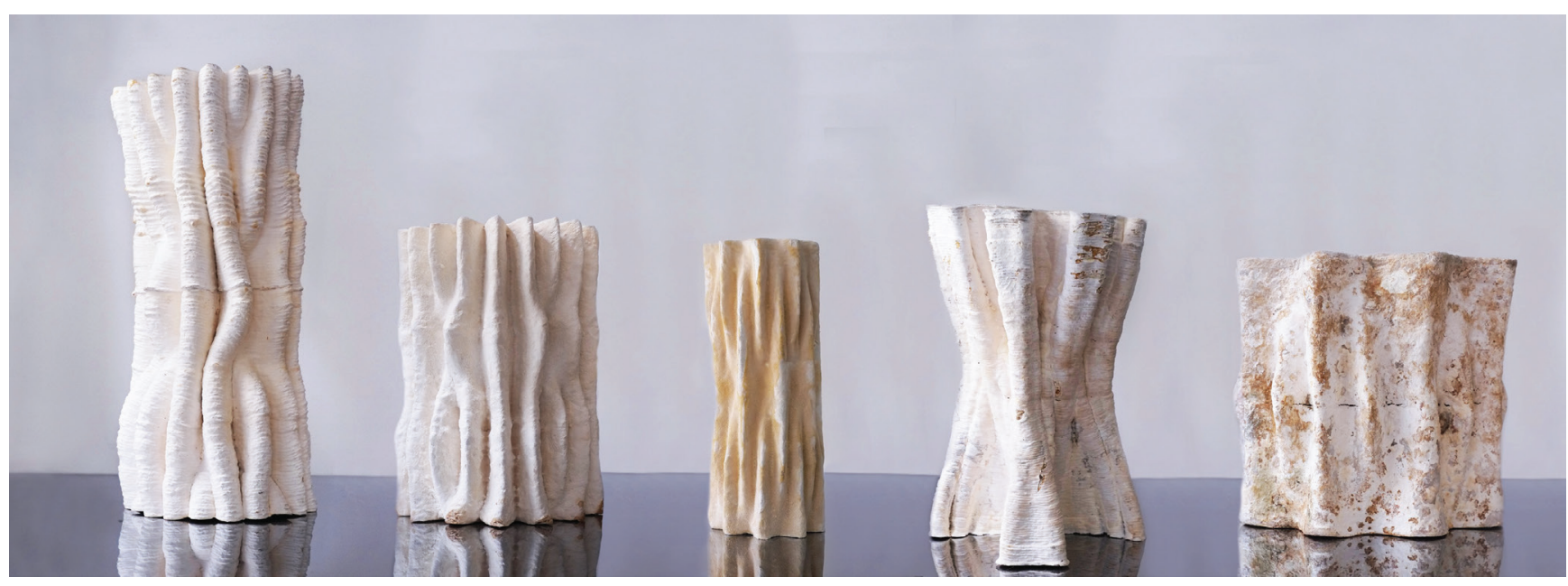

Note:From blast.studio on Facebook (https://www.facebook.com/blaststudio.biodesign/photos/a.114460489904580/282760479741246) 


\section{D/4D PRINTING AS A PRODUCTION TECHNIQUE}

In this study, the author planned to develop a bio-composite material suitable for $3 \mathrm{D} / 4 \mathrm{D}$ printing of haute couture garments, and thus this background research is broadened to investigate 3D/4D printing in more detail.

\section{INTRODUCTION TO 3D PRINTING}

$3 \mathrm{D}$ printing is a well-known chemical manufacturing technology that is used by different researchers, producers and users of fabricated 3D items using various software packages (Shin et al., 2017). The highly customised nature of 3D printing is being adopted and extended in various fields such as fashion, textiles (Pei et al., 2015), jewellery (Yap \& Yeong, 2014), supercapacitors, etc. (Stanton et al., 2015). Different chemical manufacturing technologies have been developed to process pure polymers and polymer nanocomposites (Farahani et al., 2016) for application with a range of printers, digital light projection (DLP), stereolithographic, direct inject and extrusion-based printing also known as liquid deposition modelling (Mu et al., 2017). $3 \mathrm{D}$ printing applications can be utilised for a vast range of fields such as manufacturing, construction, architecture, etc. Furthermore, active smart materials which respond to external stimuli which are used in shape recovery devices and actuators, are gaining considerable research interest. (Liu et al., 2016). 


\section{EVOLUTION FROM 3D PRINTING TO 4D PRINTING}

3D printed objects that are altered over time by environmental stimuli are now commonly referred to as 4D printing. The shapeshifting qualities of 4D printing are also being used in a range of industries such as smart textiles, robotics, biomedical devices, tissue engineering, and drug delivery (Meng et al., 2012). Natural plant structures also demonstrate this process, where different types of organs like tendrils, bracts, plants, and flowers respond to ecological impetuses of humidity, light, or touch by carrying inner turgor (the normal state of turgidity and tension in living cells), which leads to active shapes governed by the composition of tissues and cell walls in microstructural anisotropy (Nkomo, 2018).

4D printing was initially introduced by a research group at the Massachusetts Institute of Technology (MIT).

Pei (2014) and Molloy (2017) have introduced three key aspects that should be fulfilled for any 3D printing process to evolve into 4D printing.

The impetus-reactive complex materials should be selected as the manufacturing material to produce 4D printing. The selected impetus-reactive complex material needs to blend or incorporate multi-materials, with various properties being packed in layer upon layer.

The impetus will be performed on the item, causing it to animate. For example, the item may comprise heating, cooling, ultraviolet, gravity, magnetic energy, water, wind, or even humidity.

Simulation time to deliver the final result could be varied by changing the conditions of the object. 


\section{SMART MATERIALS FOR 4D PRINTING}

Smart materials are a vital aspect in the development of research in 4D printing. Their ability to change colour and rigidity or transparency are essential aspects in the process of 4D printing. (Lendlein, 2010). Different kinds of impetus can trigger time-related transformations in shape, property and functionalities. Some researchers have used water-activated 4D printing biomimetic structures inspired by nature (Gladman et al., 2016).

Other types of impetus can be used in 4D printing, such as heat (Ge et al., 2016), pH (Nadgorny et al., 2016), a mix of heat and water, as well as a mix of light and heat. The complex structures may change various arrangements due to buckling, pneumatic changes, controlled creasing, stress-induced curing, photo-induced folding, thermal controlled swelling, and usage of shape memory composites (Sundaram, Kim, Baldo, Hayward, \& Matusik, 2017). Table 1.1 presents some of the smart materials typically used, their special characteristics, and industrial applications.

TABLE 1.1

Typical Smart Materials Used in 3D/4D Printing

\begin{tabular}{|l|c|c|l|}
\hline \multicolumn{1}{|c|}{ Materials } & Input/Impetus & Outcome/Result & \multicolumn{1}{c|}{ Application } \\
\hline Polymeric gal & pH change & Swelling/ contracting & Artificial muscle \\
\hline $\begin{array}{l}\text { Electro-rheological } \\
\text { fluid }\end{array}$ & Electric signal & Viscosity & Torsional steering system damper \\
\hline Pyroelectric material & Temperature & Electric gel & Personal sensor \\
\hline Polymer & Humidity change & Capacity/ resistance change & Humidity sensor \\
\hline Self-healing materials & Force & Force & Smartphone chassis \\
\hline Smart metal alloys & Temperature & Shape & Motor actuators \\
\hline Dielectric elastomers & Voltage & Strain & Robotics \\
\hline Ceramics & Current or template & Resistance & Thermistor/Overcurrent protector \\
\hline Varistor & Voltage & Resistance & Surge protector \\
\hline Piezoelectric material & $\begin{array}{c}\text { Deformation/Strain } \\
\text { electric signal }\end{array}$ & Electric signal & $\begin{array}{l}\text { Active noise control devices, pressure and vibration } \\
\text { sensitising }\end{array}$ \\
\hline
\end{tabular}

Source: (Khan et al., 2015) 


\section{DIFFERENT APPROACHES TO 4D PRINTING}

Researchers have experimented at different levels to obtain perfect 4D printed outcomes through the manipulation of 3D printed objects.

\section{EXPERIMENTAL APPROACH}

The researcher developed and demonstrated 3D printing using different material properties. In the experiment, called "self-assembly lab", one part of the material remained rigid and another part was expanded to 100 percent of its previous volume. The expanding materials were configured into the main structure, being placed strategically to produce joints that strained and folded (Ramesh et al., 2018).

Figure 1.15 illustrates the self-assembly process of objects printed on a 3D printer. The researcher established a geometric code base on the designed object such as dimensions and angles. He also developed codes for how the object needed to change shape with the impact of outside forces of swelling through immersion in water and changes of temperature (Ramesh et al., 2018).

\section{SKYLAR TIBBITS' APPROACH}

Skylar Tibbits is a leading researcher into 4D printing, and his experiments have demonstrated that this technology is now a reality. In 2018 , he developed a 2D grid structure that distorts automatically by decreasing the dimensions across a 3D exterior (Ramesh et al., 2018).

FIGURE 1.15

4DP examples that show the single strand evolving of initials "MIT" into "SAL"

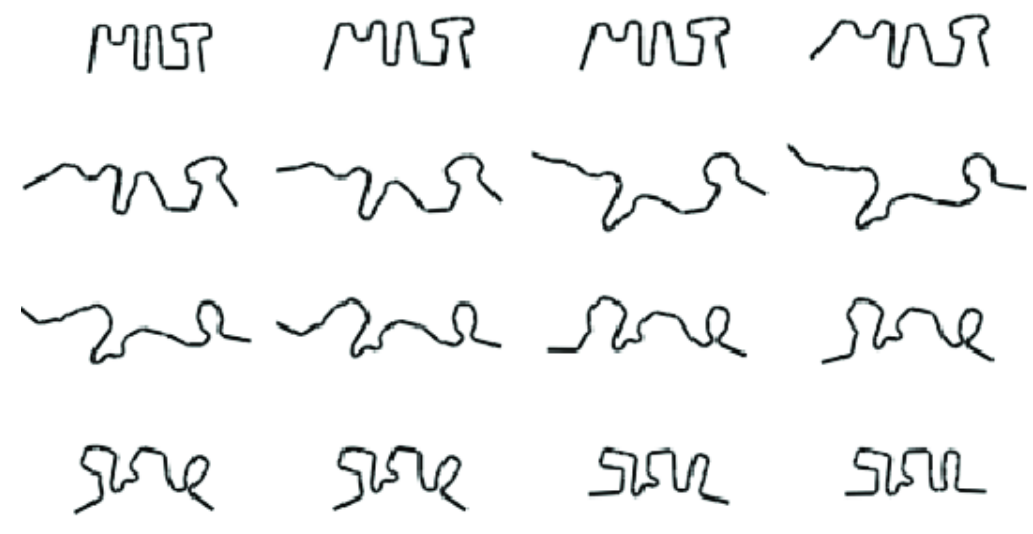

Note: From Advancements in the Research of $4 D$ Printing- $A$ Reviewby Ramesh S. (2018) on ResearchGate

(https://www.researchgate.net/figure/4DP-examples-that-show-th e-single-strand-evolving-of-initials-MIT-into-SAL_figl_326026127) 
Skylar Tibbits states that developing this concept into large scale is difficult but says at least some companies are interested in it. Stratasys is one such company that is helping Skylar in his field of research. This is a technology where a single print with different material features can transform from a 2D or 3D shape into another form using the Object Connex multi-material 3D printing technology, as shown in Figure 1.16. An important part of Tibbits' work allows researchers to program different material properties into various particles of designed geometry and obtain different water-absorbing properties of materials to activate the self-assembly process. Here the activation technology is water. This activation technology has new possibilities for including programmability into non-electronic materials, for example, robot-like behaviour without having to depend on complex electro-mechanical devices.

Some advantages of this technique of using

water-absorbent materials include:

- The chemical structure is not changed

- The physical structure as required can be programmed to change as required

- Some water-absorbing polymers can absorb up to 300 times their own weight

- It is low density

- It is highly effective

\section{FIGURE 1.16}

Stratasys Object Connex Multi-material 3D Printer

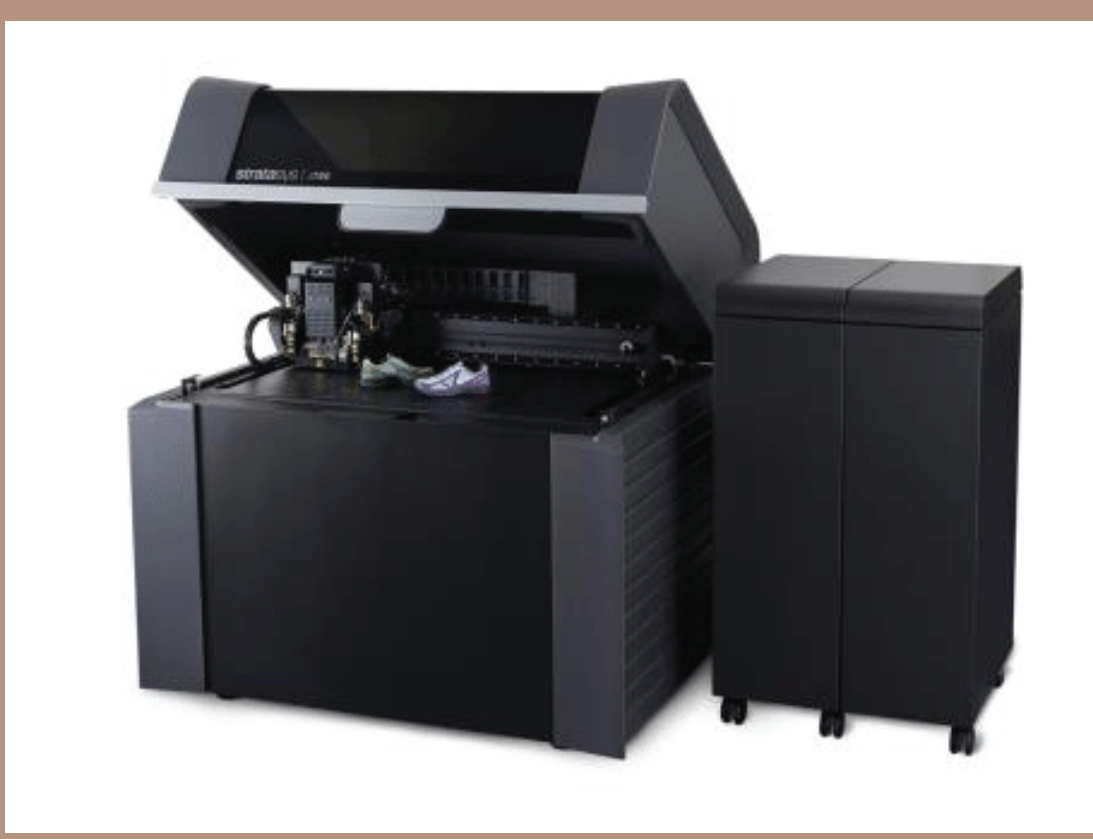

Note: From Advancements in the Research of 4D Printing-A Reviewby Ramesh S. (2018) on ResearchGate

(https://www.researchgate.net/figure/Stratasys-object-connexmulti-material-3D-printercurtsey-www3dersorg_fig2_326026127): 


\section{DEVELOPMENT OF 4D PRINTING BY JERRY QI}

Qi's research group at Georgia Institute of Technology incorporates 'shape memory' polymer fibres into composite materials and develops unique printable active composites. The initial formation is placed in precise locations of exact shape-memory fibres that will behave in their own way when exposed to an external stimulus. Shape memory is not a new concept; it is an example of self-assembly wherein the object remembers the shape and deforms by responding to the conditions.

\section{HOW QI'S 4D PRINTING TECHNOLOGY WORKS}

The research team designed specific fibre architecture at the laminate level for several composites. Composites were created with interesting thermomechanical behaviour based on the fibre architecture, size, orientation, spatial variation, and many other such parameters. Qi called these (as cited in Ramesh et al., 2018), printed active composites (PACs), which are soft materials consisting of glass polymer fibres that reinforce an elastomeric matrix. These fibres are influenced by the shape-memory effect used to create the active part of composites.

The PACs are then thermo-mechanically programmed to take 3D configurations (shapes). Change in shape is controlled through the design of inhomogeneities at the micrometric level. The complete 3D design of fibres and matrix are printed from a CAD file using an object Conex 260, 3D printer. Then a process that results in a layer that contains matrix and fibre is undertaken by depositing the polymer ink droplets at about 70 degrees $\mathrm{C}$, wiping them into a soft, smooth film and later having them UV photopolymerised. The entire composite design is then analysed by printing multiple film layers to create individual lamina, multiple laminas and later, the 3D laminate. 
Advantages of 'shape memory' polymers:

- Good recovery strain

- Low density

- Cost less

Drawbacks of 'shape memory' polymers:

- Relatively low modulus

- Low strength

TABLE 1.2

Properties of 'shape polymers' from Qi's experiment

\begin{tabular}{|l|l|}
\hline \multicolumn{1}{|c|}{ 'SHAPE MEMORY' POLYMER PARAMETERS } & \multicolumn{1}{c|}{ RANGE } \\
\hline Density (g/cm3) & $0.9-1.2$ \\
\hline Extent of deformation & Up to $800 \%$ \\
\hline Required stress for deformation & $01-$ Mar \\
\hline Stress generated upon recovery & $01-$ Mar \\
\hline Transition temperature & -10.1 \\
\hline Recovery speed & 1 s-min \\
\hline Processing condition & $<2000$ c low pressure \\
\hline Cost & $<\$ 10 /$ lb. \\
\hline Shape recovery ration & Recovery ratio upon bending is above $90 \%$ \\
\hline
\end{tabular}

Source: (Ramesh et al., 2018) 


\section{HAUTE COUTURE FASHION}

Barchfield's study of Couture in Crisis in 2009 (as cited in Latter \& Marchegiani, 2010) describes haute couture as the prestigious front for French creative fashion, where extremely priced garments are made to measure for an elite cadre of the world's ultra-rich customers. Here, designers unleash their creativity using the finest materials and techniques.

Clerk (2008, p. 440) distinguishes haute couture as the pinnacle of the fashion system, which can offer something specific to an individual in terms of fit and appearance. He acknowledges that haute couture garments and accessories are investments, with both economic as well as emotional significance.

The prime objective of the current research is to develop a series of experimental materials and $4 \mathrm{D}$ printing techniques that might illustrate more sustainable methods of producing haute couture fashion. In the following sections, manufacturing techniques discussed in previous sections will be elaborated further, in the context of haute couture fashion. 


\section{D/4D PRINTING IN HAUTE COUTURE FASHION}

With technological advancements, new perspectives and applications have evolved in fashion design, through the adoption of widespread techniques such as digital printing and laser cutting in textiles and, more recently, 3D printing in clothing production (Porter, 2017).

Advantages from the same technological advancements have been adopted by the haute couture fashion industry as well, and many designers have stepped into the phase of 3D printing their latest design collections. Alexandrea (2020) argues that 3D printing technologies have penetrated the fashion industry, particularly the haute couture sector. The design freedom enabled from additive manufacturing has prompted designers into working with 3D printing technology to create complex shapes and original pieces.

Designers from the haute couture fashion industry have introduced to the world some amazing conceptual and commercial products that have been produced using 3D and 4D printing technologies.

\section{PRECEDENCE STUDY OF 3D PRINTED HAUTE COUTURE FASHION}

Research was carried out to gain an understanding of how 3D printing technology has been used by contemporary haute couture fashion designers. A range of examples from collections has been compiled showing a variety of qualities.

\section{'HYBRID HOLISM', 'VOLTAGE' COLLECTIONS BY IRIS VAN HERPEN}

Iris Van Herpen has experimented with several 3D printing processes in her collections. She is a Dutch designer who combines new technology with traditional haute-couture techniques (Hemmings, 2020). Using 3D printing techniques, she has successfully developed ready-to-wear pieces. Incorporation of traditional construction techniques with $3 \mathrm{D}$ printing can be recognised in the collection's dresses, Hybrid Holism (Figure 1.17) (Chakraborty \& Chandra Biswas, 2020).

Here 3D printed structures were created using liquid resin and then assembled using traditional techniques. 
Polyjet multi-material printing technology was used to develop a cape and a skirt (Figure 1.18) by the designer for her 'VOLTAGE' collection. She has used multiple materials, both soft and hard, to create a unique texture, flexibility, and movement. The dress named 'Voltage' was developed using selective laser sintering (SLS) (Chakraborty \& Chandra Biswas, 2020).

This technique consists of melting powder particles and fusing them selectively using $\mathrm{CO} 2$ beams. The material used by the designer is thermoplastic polyurethane (TPU). The use of powder has enabled the construction of layers and intricate details. This method has resulted in the garment having an organic movement. The materials used here can be questionable, as most of them are plastic polymer derivatives that can be hazardous to the environment. Therefore, experimentation with more material is necessary to make this a more sustainable manufacturing technique.

\section{FIGURE 1.17}

A Stereolithography Dress from 'Hybrid Holism' Runway Collection (July, 2012) by Iris van Herpen

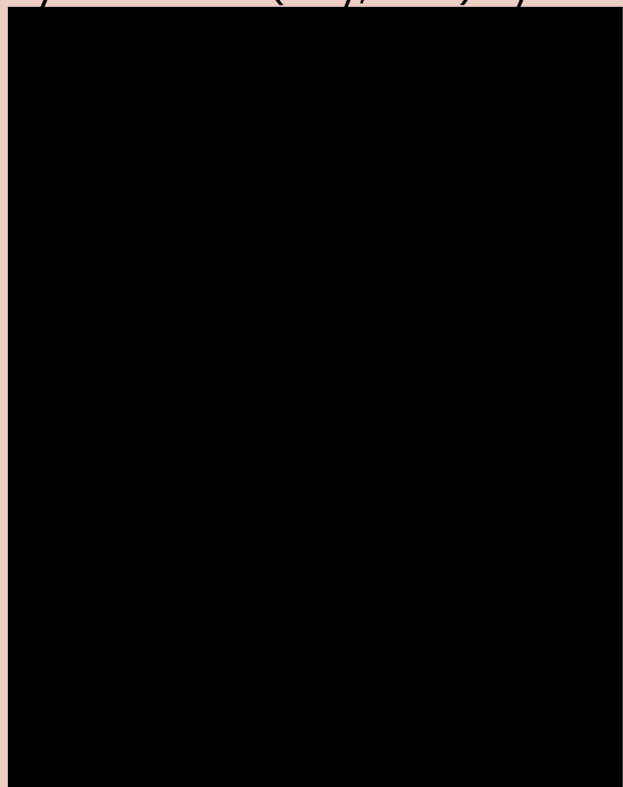

Note: From LOOK 03 of HYBRID HOLISM ON IRIS VAN HERPEN (https://www.irisvanherpen.com/haute-couture/hybrid-holism)

\section{FIGURE 1.18}

Cape and Skirt for 'Voltage' Collection (January, 2013) by Iris van Herpen

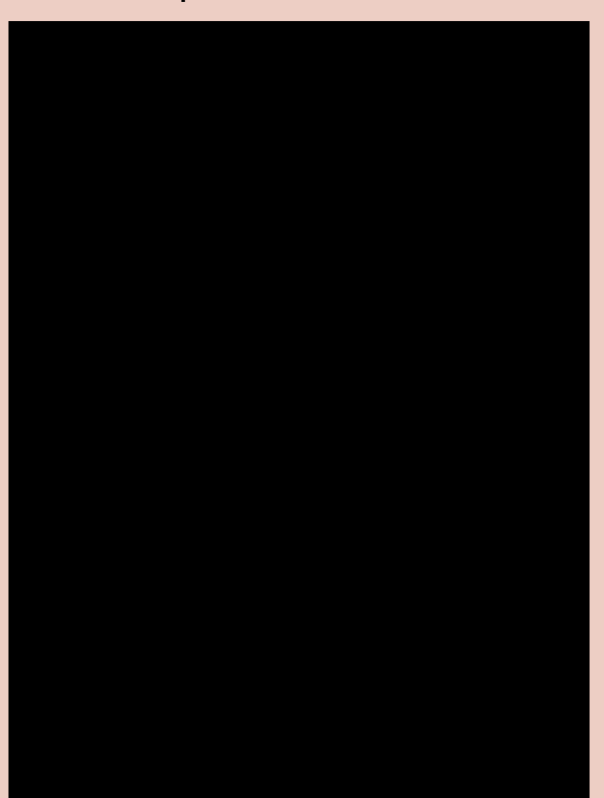

Note: From LOOK 10 of VOLTAGE On IRIS VAN HERPEN (https://www.irisvanherpen.com/haute-couture/voltage) 


\section{WORKS BY JULIA KOERNER - THE 3D STOLA}

Known as the Architecture's Queen of 3D fabrication, Julia Koerner, an award-winning Austrian designer working at the convergence of architecture, product, and fashion design, set herself apart as a designer due to her relentless quest for 3D fabrication mastery (Guimapang, 2019).

For the night of the Academy Awards 2019, she designed a customised 3D printed statement for Oscar-winning costume designer, Ruth E Carter, called 3D STOLA (Figure 1.19).

For this design masterpiece, Koerner partnered with Swarovski to integrate their cutting-edge line of crystals onto the garment in a novel technique, which is a first of its kind. Starting with a 3D scan of Ruth's body, Julia Koerner found inspiration in African imagery from the photographer Seydou Kaïta, and digitally crafted afro-futuristic patterns to an asymmetrical ensemble that references the 1950s fashion designs of Balenciaga ("3dstola", n.d.).

SLS - uses a bed of powder liquified by a microscopic laser beam and the fused powder is used in layers to generate the computer-designed 3D model. The manufacturing process was carried out by the famous 3D printing venture, Materialise, in Belgium.

FIGURE 1.19

Ruth E Carter and 3D STOLA

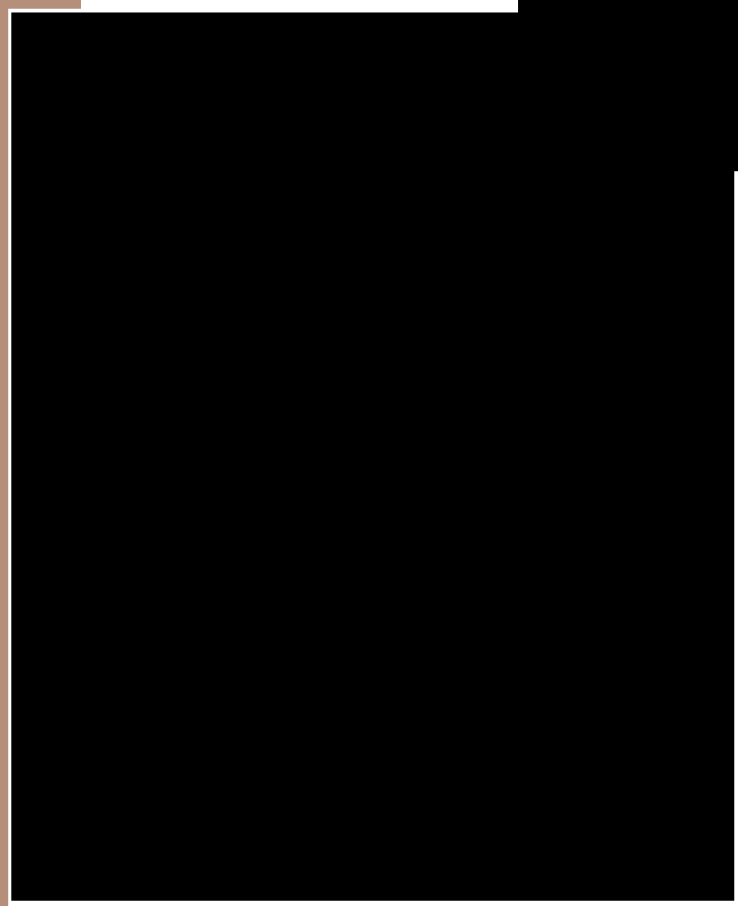

Note: From 3D STOLA, Julia Koerner on Not Just A Label (https://www.notjustalabel.com/collection/infoll30/3d-stola) 


\section{WORKS BY JULIA KOERNER - SETAE JACKET}

In designing and producing this jacket, Setae (Figure 1.20), Madagascar Sunset Butterfly's biomimicry has been explored and 2D macro scans of butterfly wings have been digitalised into an algorithm and translated into 3D patterns. Due to the movement and delicate colour transformation, it expresses a true organic animal flow that comes to life (Setae Jacket, 2019).

FIGURE 1.20

Setae Jacket by

Julia Koerner 


\section{D 'WEAREABLE' KIMONO COLLECTION BY GANIT GOLDSTEIN}

Pioneer designer Ganit Goldstein launched her latest Japanese-style 3D printed pattern collection, 'WeAreABle'. Goldstein's unique 3D design of wearable kimono collection (Figure 1.21), has followed Japanese ikat weaving - a traditional folk craft developed during the middle of the 18th century, with the unique patterns looking like splashes on the fabric (Facfox, Inc., 2020).

By combining the fact that the human body is a platform for innovation with the development of smart textiles, Goldstein has been able to re-draw the demarcation between machine-made and handmade in haute couture fashion. The soul of this project captures qualities of Japanese embroidery, through textile multi-colour 3D printing.
FIGURE 1.21

Ganit Goldstein's 'WeAreAble' Kimono Collection

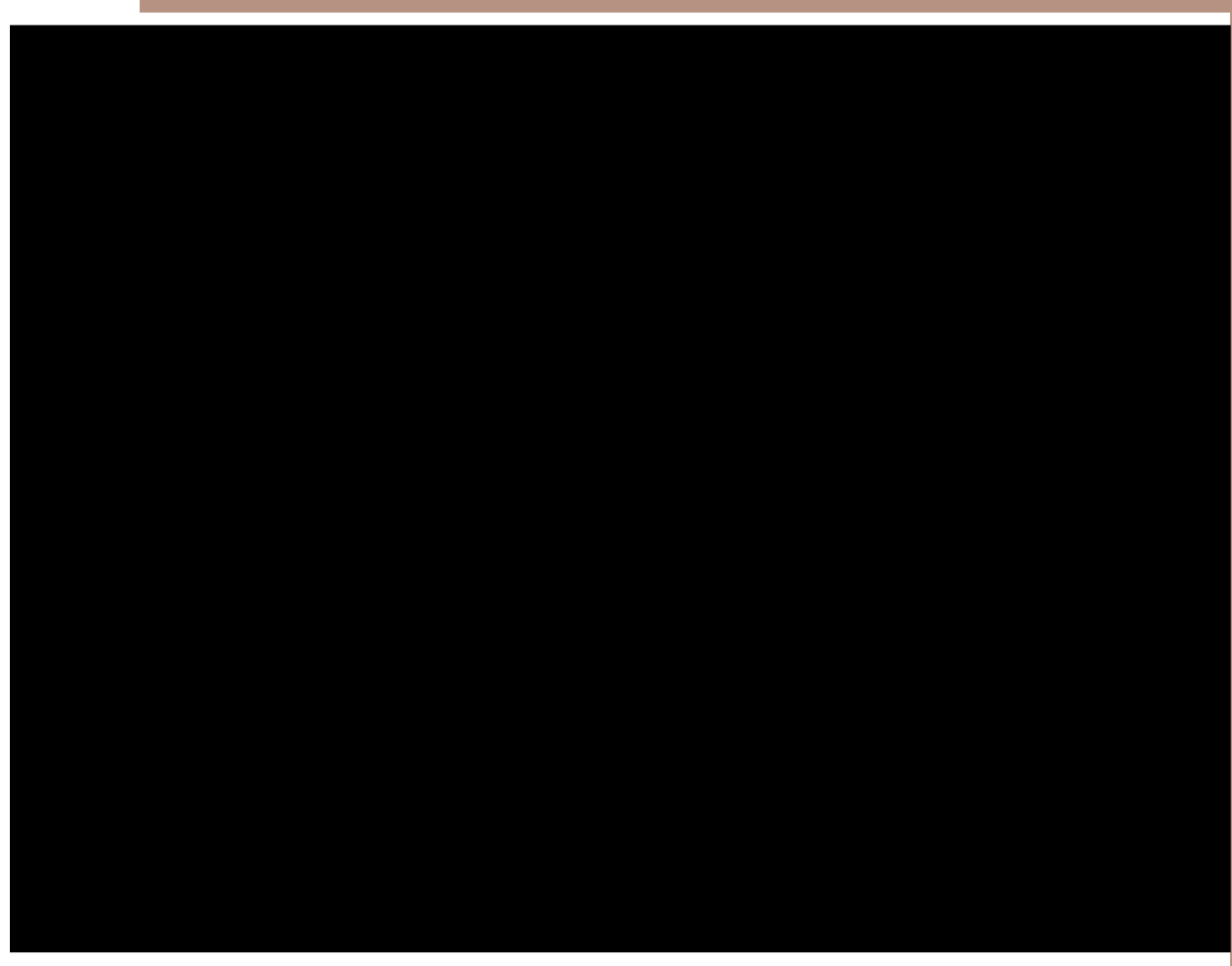

Note: From 3D 'WeAreAble' kimono collection 2020 on Ganit Goldstein (https://ganitgoldstein.com/weareable-kimono-collection) 


\section{BIO-COUTURE, THE FUTURE}

\section{PRECEDENCE STUDY OF DESIGN PORTFOLIO ON BIO-COUTURE}

This manufacturing concept and technique was first introduced to the world by Suzanne Lee, an American Senior Research Fellow at Central Saint Martin's College of Art and Design, in 2003. Through a series of experiments, Lee has managed to produce bacterial cellulose similar to leather, using a mix of different organisms. This concept of bacteria being used to produce materials that are then turned into clothes is called bio-couture. The significance of these bio-based clothing materials is that they are not only biodegradable but also fully compostable, which means that they can be just thrown away into the environment without any harm or pollution of natural resources.

Lee expresses that it is a fermentation method that grows bacterial cellulose, and one could call it vegetable leather (What is Biocouture? 2020).

Discussed below are some of the finest real-world examples of manufactured products based on bio-couture.

\section{VEGAN LEATHER AND 'MYLO' BY STELLA MCCARTNEY}

The technology of growing and using kombucha cellulose to create vegan leather has been explored over the past few years through a collaboration between the Fashion Department, Queensland University of Technology, and scientists from The Edge, State Library of Queensland.

Vegan leather is generally produced using polyurethane. Many sustainable materials could replace polyurethane to make vegan leather, including pineapple leaves, apple peels, cork, other fruit waste, and recycled plastic. Stella McCartney is a designer who uses only vegan leather for her collections. Vegan leather is used to produce a variety of products from moto jackets to intimate garments. Vegan leather is used not only for dresses but to make different kinds of shoes, boots, belts, handbags and even seat covers for some of the famous cab manufacturers (Peta, 2020). 
FIGURE 1.22

The Two-Piece Outfit by Stella McCartney's Brand MYLO Consists of a Black Bustier and Matching Trousers

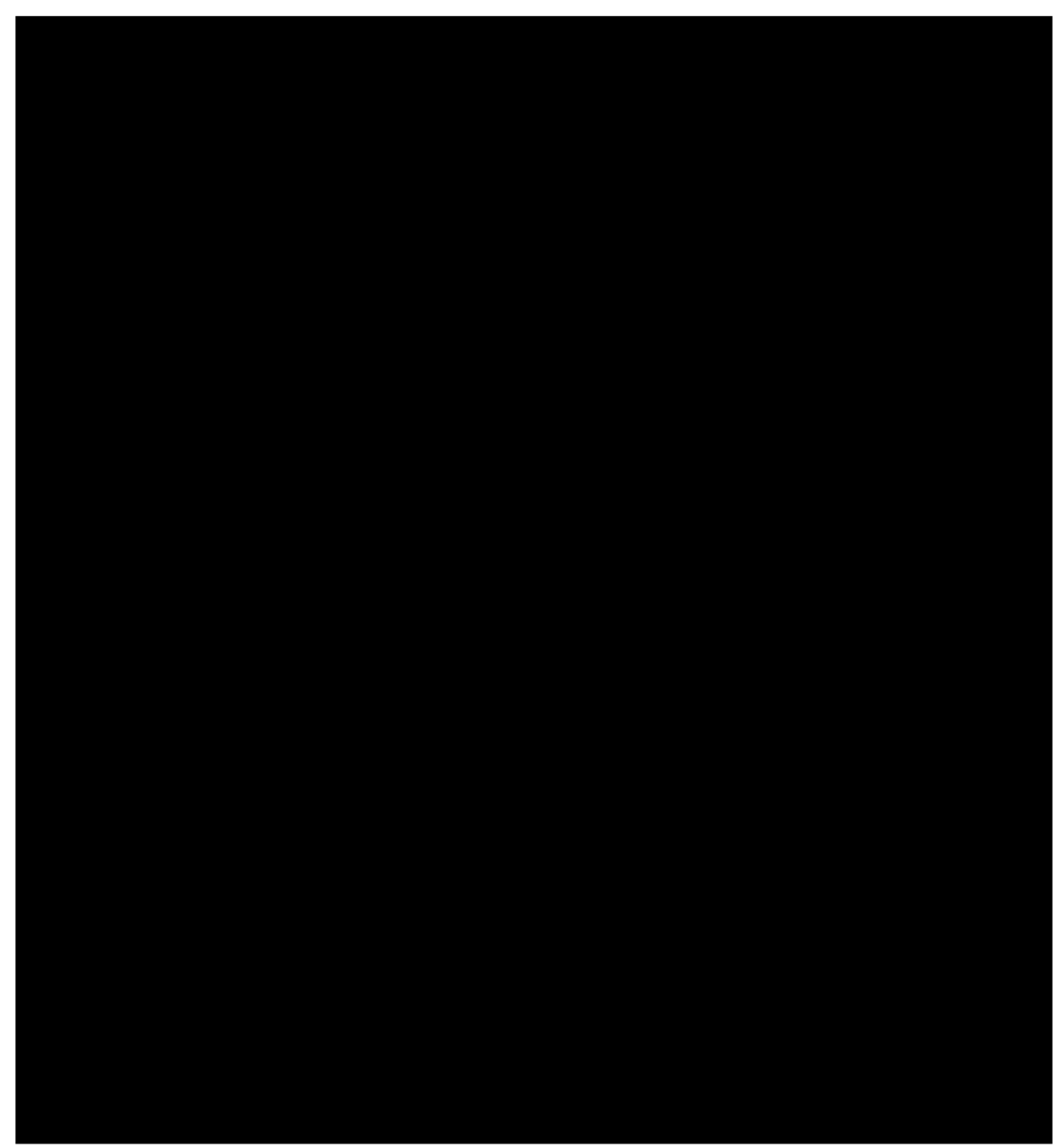

Note: From Stella McCartney creates clothes from mycelium leather to foster a "kinder fashion industry" by Jennifer Hahn on Dezeen
Eco-Friendly Faux Leather (EFFL) is a recently developed environment-friendly leather type, and it is a type of vegan practice. Its main feature is its low carbon footprint (Kim et al., 2016).

Similarly, Stella McCartney has unveiled a two-piece outfit, named MYLO (Figure 1.22), made using a leather alternative grown from mycelium, making her among the first luxury fashion designers to turn biomaterial into wearable clothing (Hahn, 2021). 


\section{GROWING SHOES BY KRISTEL PETERS}

Using Mycelium, "Growing Shoes" shows the results of experiments to see how shoes can be shaped into solid and hollow forms as in Figure 1.23. The solid shapes function as heels, platforms, and insoles of the shoe. To achieve these shapes, different waste streams such as coffee grounds and sawdust are used as substrates. The hollow shapes are grown in bottles or on fibres such as hemp and psyllium to achieve strength and structures. After harvesting, the pure Mycelium, which becomes white and looks like clay, is then used to cover some parts of the heels or moulded onto the lasts.

\section{FIGURE 1.23}

Kristel Peters' Growing Shoes

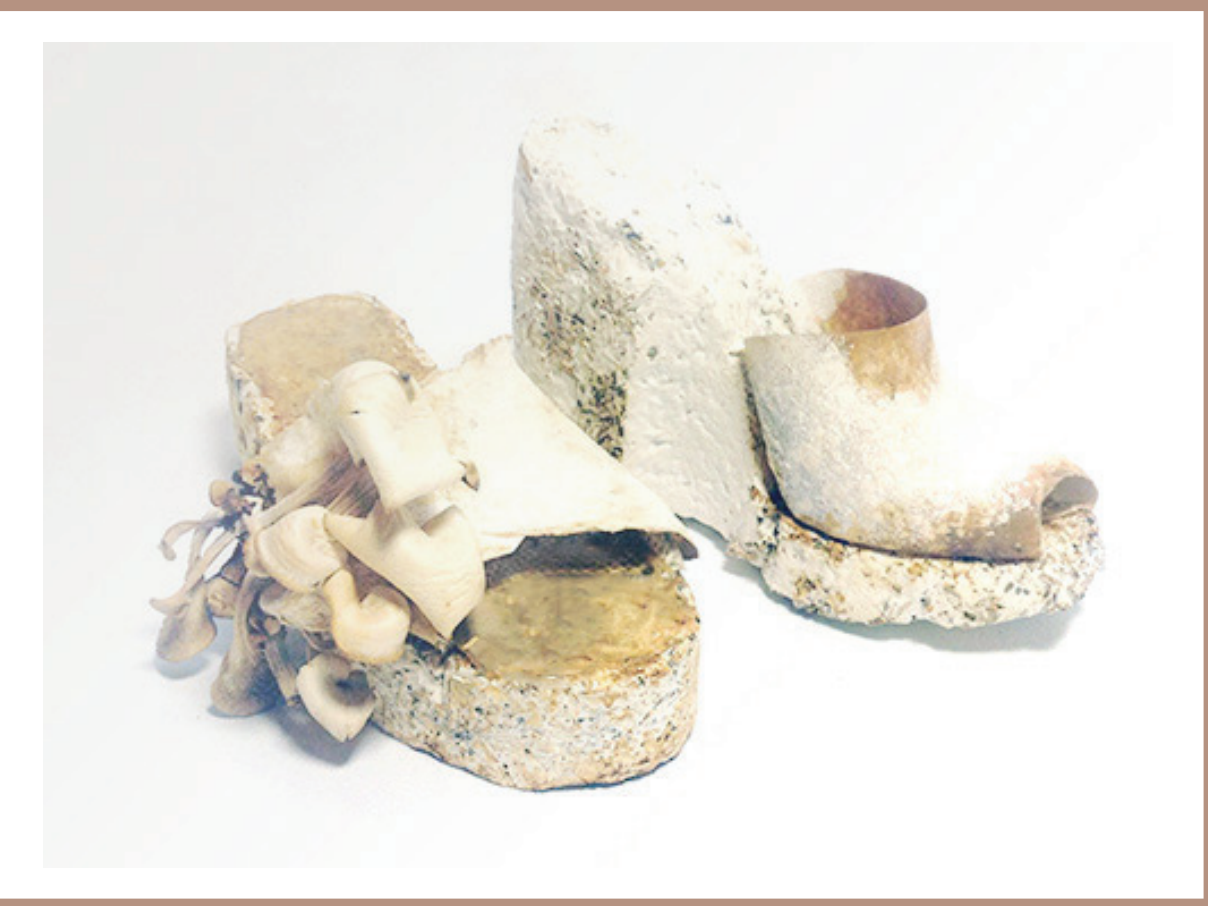

Note: From Growing Shoes - Regenerative Shoe Design by Kristel Peters on SHOE DESIGNER @Studio COJAK (http://shoedesigner.be/project/210/) 


\section{MYCO-TEX BY ANIELA HOTNIC}

MycoTEX $®$ is a groundbreaking seamless manufacturing method allowing for custom-made clothes made from compostable mushroom roots. (MycoTEX", 2020)

Mycelium (the root of a mushroom) has been predominantly used in a solid state in combination with a substrate. However, Aniela Hotnic started by combining mycelium (and its peculiar properties) with textiles to create flexible composite products, learning while researching; her goal turned into developing textiles consisting exclusively of pure mycelium.

FIGURE 1.25

Material Development Experiments for MycoTEX $\AA$

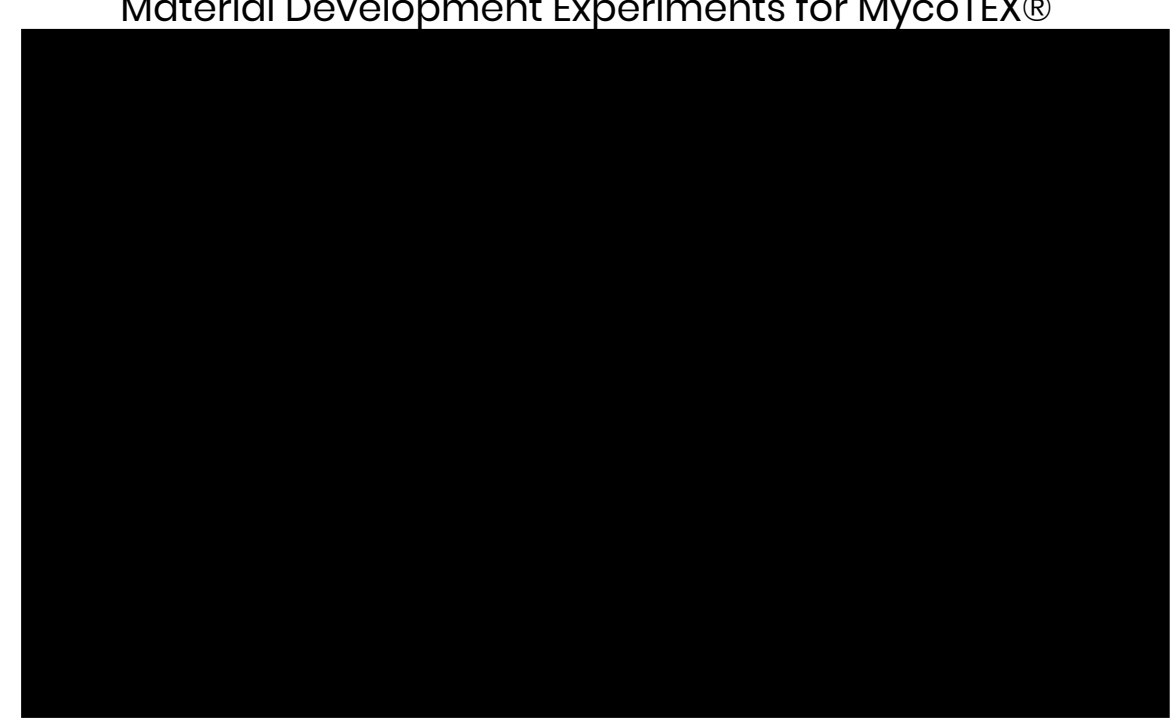

Note: From MycoTEX proof-of-concept on NEFFA (https://neffa.nl/portfolio/mycotex/)
FIGURE 1.24

Products Made of 'Seamless' MycoTEX $\AA$

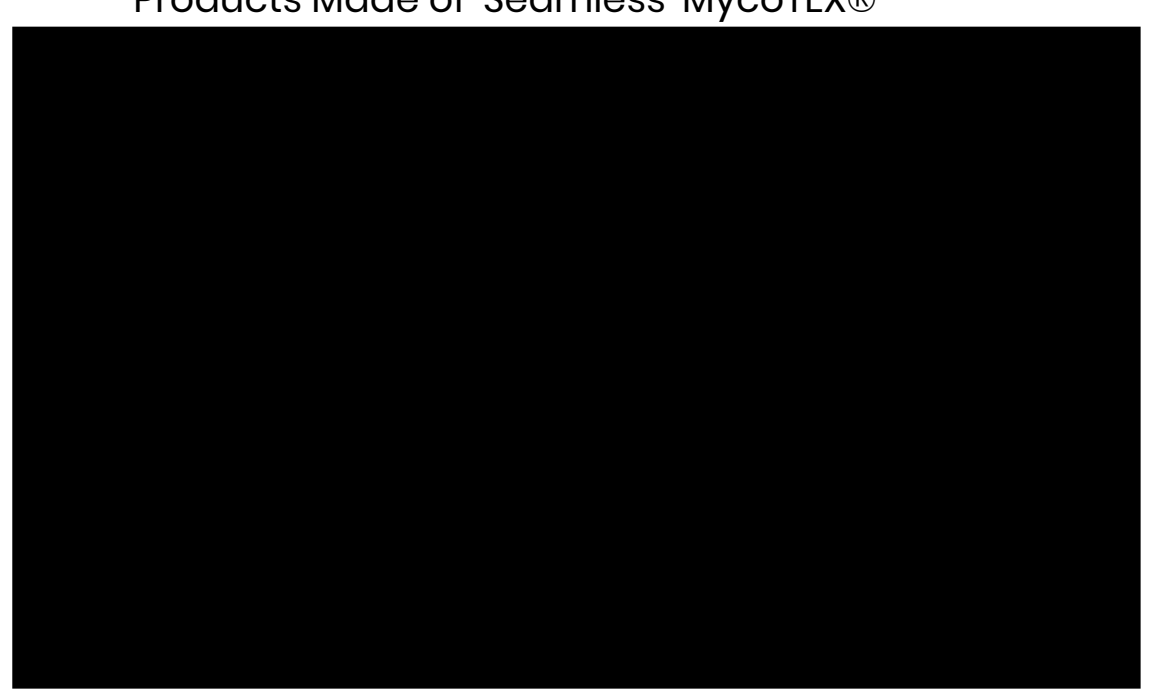

Note: From MycoTEX proof-of-concept on NEFFA (https://neffa.nl/portfolio/mycotex/) 


\section{BIOBOMBER JACKET BY SUZANNE LEE}

Suzanne Lee has been experimenting with a new approach to textile manufacturing (Growing.it, n.d).

The growth process is quite simple, as it only requires some green tea, sugar, and microbes. When these materials are mixed, they form a static culture and no complex processes of spinning, weaving, or printing are involved. The sheet is then dried and cut or shaped and moulded into clothes and accessories.

Note: From Suzanne Lee Grows Her Own BioCouture Bomber Jackets by Katie Levitt on VICE

(https://www.vice.com/en/article/epnd7e/seaweed-cloth es-can-save-the-planet) 


\section{THE COUTURE DRESS WITH ALGAE SEQUINS BY PHILLIP LIM AND CHARLOTTE MCCURDY}

Taking the concept of sustainable design to the next level, fashion designer Phillip Lim collaborated with industrial designer Charlotte McCurdy in designing and developing a dress using bioplastic sequins, free from petroleum products like synthetic fibres, dyes, and plastic sequins. According to McCurdy, as cited by Hornsby (2021), sustainable fashion is not just about using only organic, natural or recycled materials, but at the same time ways and means need to be invented to replace 60 percent of textiles that are currently made of fossil fuel-based materials to bring down carbon emissions to zero.

To cater to this requirement, bioplastic fronds made from algae are sewn onto a biodegradable base layer that is made from plant fibres in producing this innovative dress design (Figure 1.27).

\section{FIGURE 1.27}

The Adorn Couture Dress Made with Algae Sequins

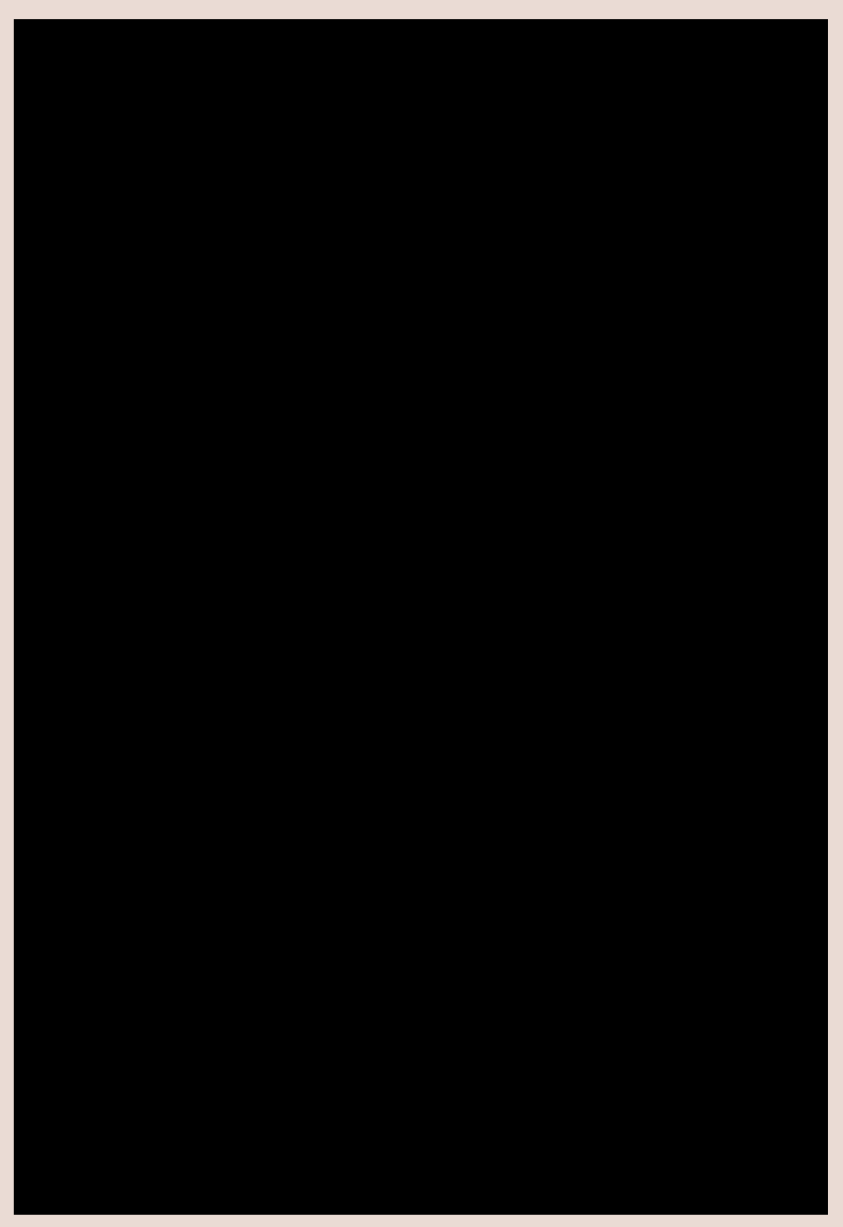

Note: From Phillip Lim and Charlotte McCurdy. Adorn couture dress with algae sequins by Amy Hornsby

(https://dailycharlottenews.com/phillip-lim-and-charlotte-mccur dy-adorn-couture-dress-with-algae-sequins/) 


\section{DIRECTION OF STUDY}

In summarising, 3D printing technologies adopted in the haute couture fashion arena have created many opportunities for creative design and innovation. However, the materials being used in generating these products are not so eco-friendly and sustainable, as their key compositions are still petroleum-based products.

The use of biomaterials is still constrained only to products with natural growth and has very limited control for pre-defined, automated production.

Research findings through this literature survey have revealed a literature gap for a bio-composite material that could be used in sustainable 3D printing. The research was conducted following the methodology discussed in the next chapter, to develop a sustainable bio-composite material, perform 3D/4D printing and adopt the outcomes into the world of haute couture fashion. 
RESEARCH METHODOLOGY $\checkmark$ INTRODUCTION

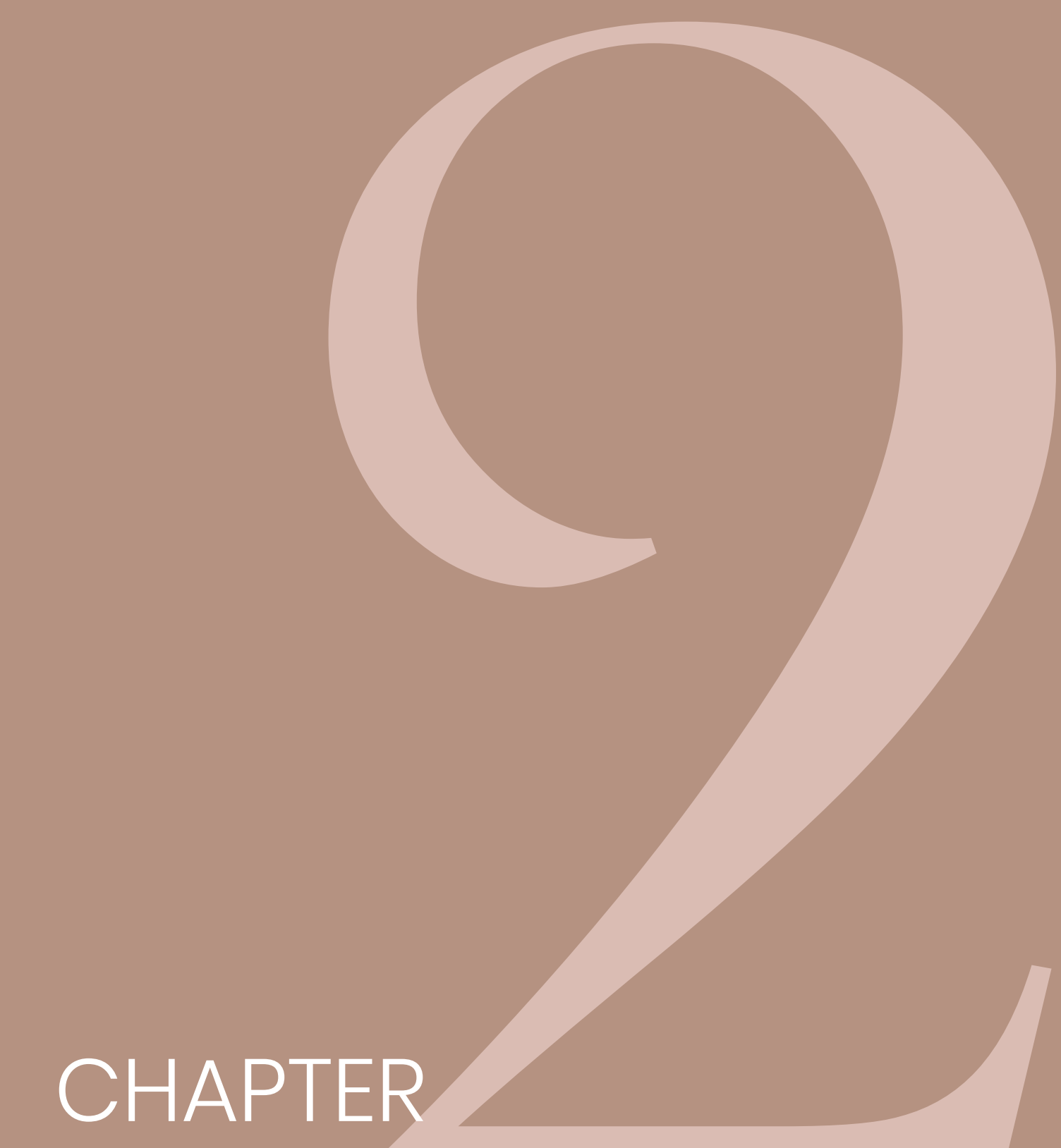




\section{RESEARCH METHODOLOGY}

\section{INTRODUCTION}

Rajasekar et al. (2013) emphasised that "research" is a systematic and logical search for innovative and useful information on a particular subject. They further elaborate that success in research is achieved through studies, experiments, observations, analysis, comparison, and reasoning.

Methods and methodology are two distinguished, yet closely related aspects of research. According to Saunders (2007), methods are the techniques and procedures used to obtain and analyse research data, and a methodology is the designing of how research should be conducted using the adopted research methods, based on theoretical assumptions.

The significant decision in the process of designing research is the approach that a researcher would elect, as it determines how the relevant information for a specific study is obtained (Sileyew, 2019).

Buchanan and Friedman's study of design research (as cited in Frankel $\delta$ Racine, 2010) explained that the approaches to new design knowledge could be divided into three categories; basic research, clinical research, and applied research. 
Basic research, which is also known as research about design, is the most commonly practiced research methodology, where one would simply research on existing designs to get inspired as well as to study the pros and cons (Frankel \& Racine, 2010).

Clinical research, which is more advanced compared to basic research, is the practice of researching to develop the design. Activities like observation, measurement, interview, literature review, analysis, and validation fall under this type of research, where different research methods are applied during these activities. To simplify this theory, Stappers \& Giaccardi (n.d.) used the term "design informed by research", which refers to the gathering and application of relevant scientific and technological information and conducting studies to learn specific information about the situation for which the design is made.

Applied research, also defined as research through design, is where practitioners research to broaden their knowledge and understanding of a specific product or area, using a designed prototype, by varying its interrelated characteristic properties.

On that front, the methodological approach adopted for the current study, in conducting the entire research work, is described in the next section. 


\section{RESEARCH PROBLEM}

With the current trends of sustainable development and environmental concerns, the world is moving towards innovating products that could withstand the challenging future through effective design and eco-friendly biodegradable materials. Similarly, many researchers, architects, designers, and scientists are working in collaboration to advance sustainability within the fashion industry.

At present, sustainable bio-composite materials are being introduced into the field of fashion design, but they are often limited by the level of controllability used in precision-oriented production processes.

In this context, I was intrigued to find solutions to two of the prime research aims described below.

\section{AIM 1:}

To develop a sustainable bio-composite of a semi-liquid nature suitable to be used as a 3D printing material.

\section{AIM 2:}

Perform 3D printing using the developed material and adopt the outcomes into haute couture fashion.

The research was conducted using qualitative data and the primary data collection was done by myself through experiments by manipulating variables. 


\section{METHODOLOGY}

\section{FIGURE 2.1}

Research Problem, Aims, Methods and Methodology

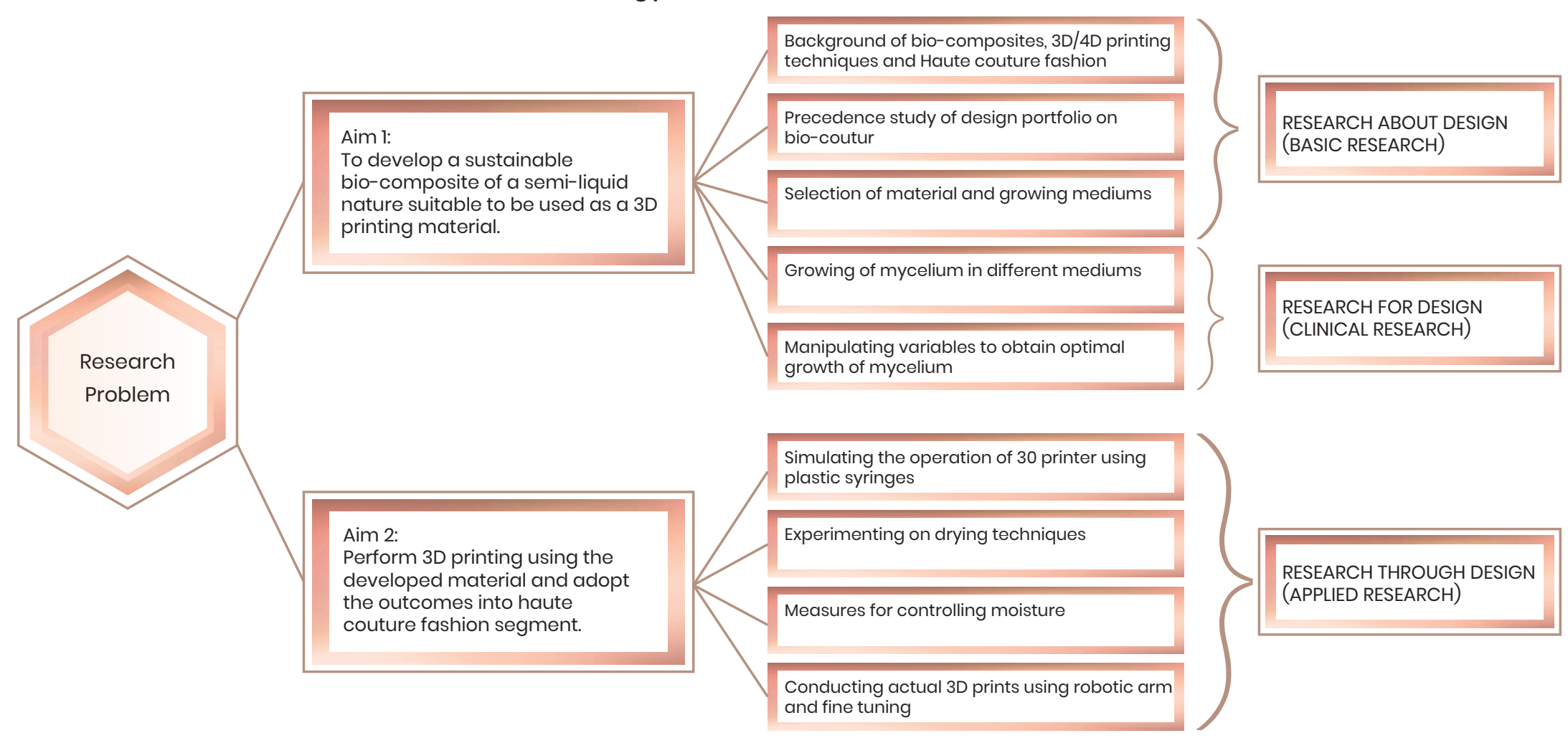

In compiling this thesis, I used all three knowledge approaches on research and design, as explained by Frankel \& Racine (2010), in different phases. Figure 2.1 illustrates the inter-relationships amongst aims within the different phases of the research and the integration of the applied knowledge-gaining approaches.

Note: Author's work (2021) 


\section{MATERIAL DRIVEN DESIGN (MDD) METHOD}

Through their experience gained by working across many material-based experimental projects and the knowledge gathered on materials and design domain, Karana et al. (2015) introduced this method to facilitate the design processes in which materials are the main drivers.

As further described by Karana et al. (2015), this method could be applied in the following three scenarios in material-based experiments.

1. Designing with a relatively well-known material. Seek new application areas to evoke new meanings and to elicit unique user experiences.

2. Designing with a relatively unknown material, affording the designer opportunity to define application areas through which unique user experiences, identities for materials, and new meanings may be introduced.

3. Designing with a material proposal with semi-developed or exploratory samples, which needs the designer to propose meaningful applications through which unique user experiences and meanings will be elicited.
There are four sequential action steps in the MDD Method that could be illustrated as in Figure 2.2.

\section{FIGURE 2.2}

Material Driven Design (MDD) method

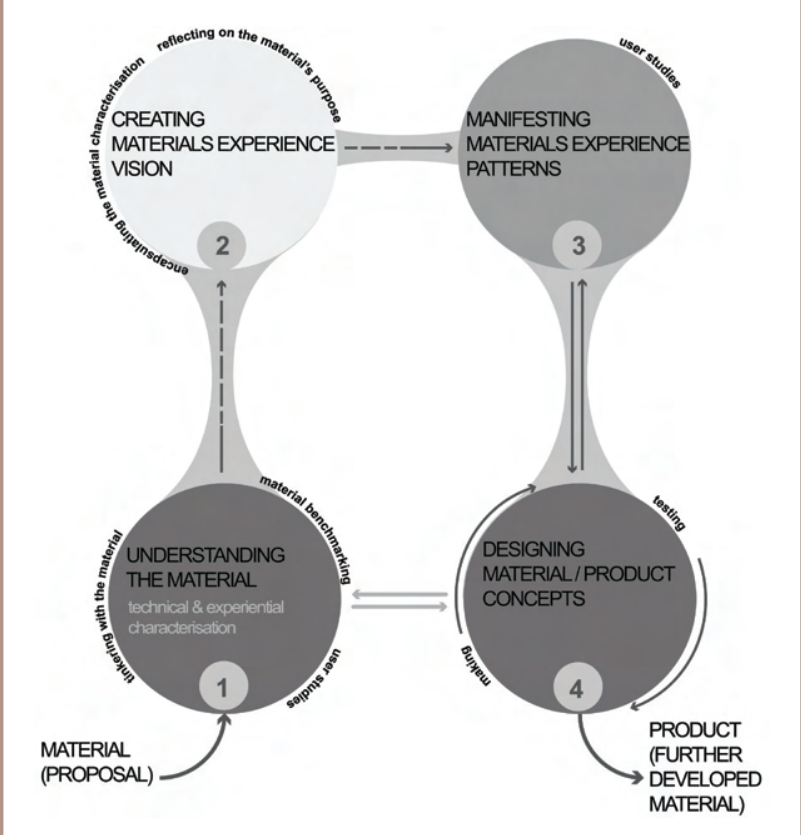

Note: From Material Driven Design (MDD):

A Method to Design for Material Experiences by Karana et al. (2015)

The next chapter discusses in detail how the knowledge gathered on research methodologies were applied in a practical context. The design and conducting of the experimental research followed the MDD Method. 

GROWING MATRIX AND SYRINGE PRINTING
EXPERIMENTS 


\section{GROWING MATRIX AND SYRINGE PRINTING EXPERIMENTS}

\section{INTRODUCTION}

The objective of the experiments was to obtain a sustainable bio-composite material from mycelium, which had the desired workability parameters to be used as a 3D printing material. In working towards the objective, I used variants of mycelium and followed different growing techniques.

Observations were photographed and recorded to examine the successful mycelium growth over time.

To adopt the most suitable mycelium variant, growing medium, and growing techniques, a stage-by-stage experimental approach was used, based on information obtained from the Literature Survey. 


\section{SELECTION OF BIO-COMPOSITE MATERIALS}

The selection of a sustainable bio-composite material primarily focused on two parameters: One, that it should exhibit sufficient growth over time and two, it should possess a desired viscous level, so that it could be used as a 3D printing medium. The study of these parameters was conducted in two different phases, as described below.

Mycelium is becoming a commonly used bio-composite material for conducting design experiments of this nature. In this study, five variants of mycelium were experimented with, using different growing mediums, to observe the rate of natural growth, length of growing time, and to ascertain the most productive conditions for growing mycelium material. As previously mentioned, mycelium is the root system of a mushroom, consisting of the growing stem cells of the fungus.

To examine the nature of growth of different variants of mycelium, three commonly available bio-composite materials, from the agricultural sector, and two native cultures from Manaaki Whenua-Landcare Research's
International Collection of Microorganisms from Plants (ICMP) were selected.

While mycelium from oyster mushrooms, ganoderma and turkey tail mushrooms were chosen as commonly available international varieties, the cultures received from Manaaki Whenua were native to New Zealand and were of interest in assessing the performance of indigenous mycelium.

Of these, the oyster mushroom is the most common edible mushroom type and it is grown commercially around the world to fulfil food requirements (Valverde et al., 2015).

Ganoderma is a species that belongs to the Ganodermataceae family, and it is a genus of polypore fungus. It has about 80 species, with these being mainly distributed in tropical regions, and these have great medicinal and economic value (Hapuarachchi, 2018). Tukey tail is a medicinal type of mushroom and it has powerful antioxidants and other compounds (Kubala, 2018). 
FIGURE 3.1

Oyster Mushrooms

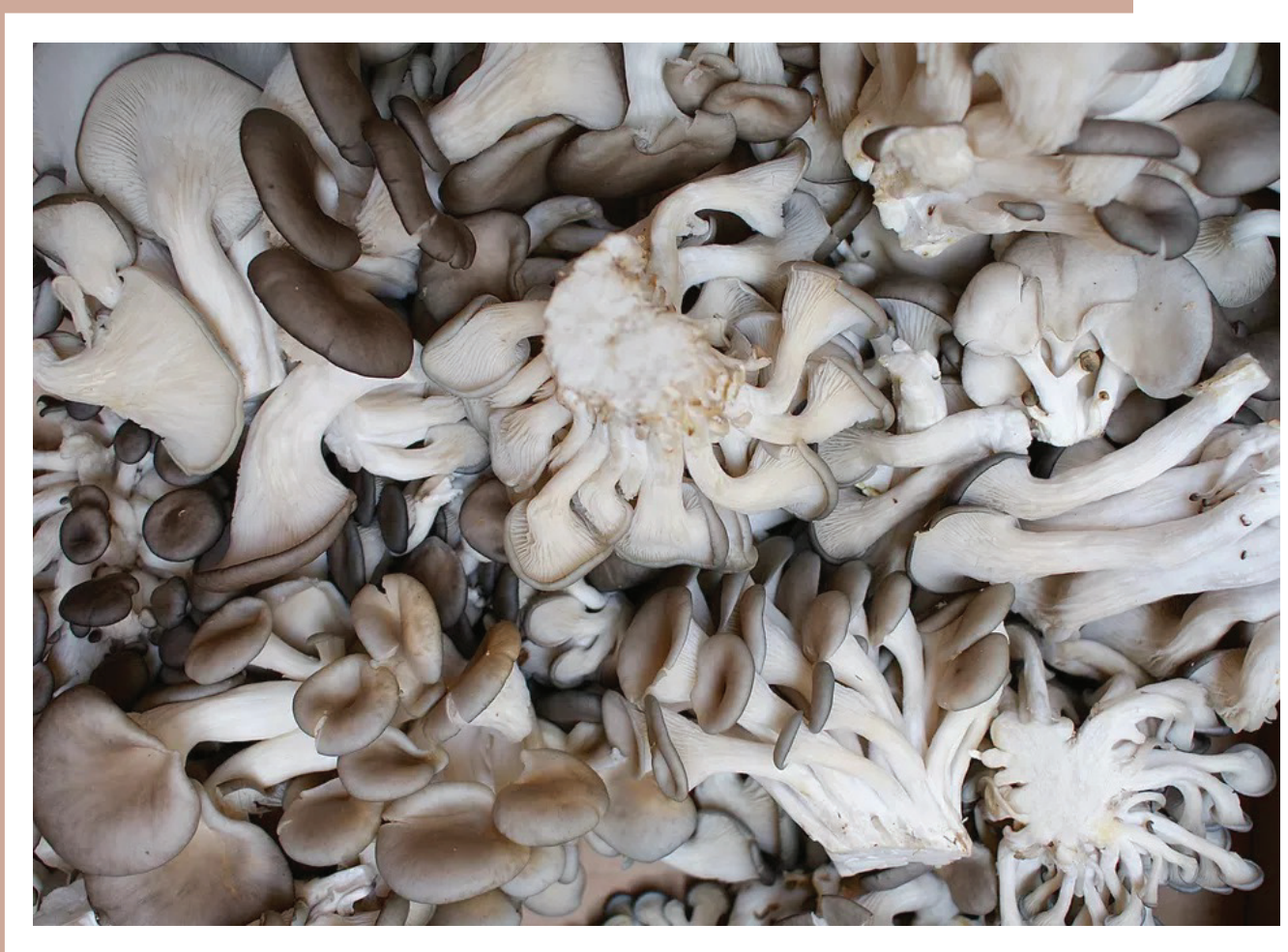

FIGURE 3.2

Ganoderma and Turkey Tail Mushrooms

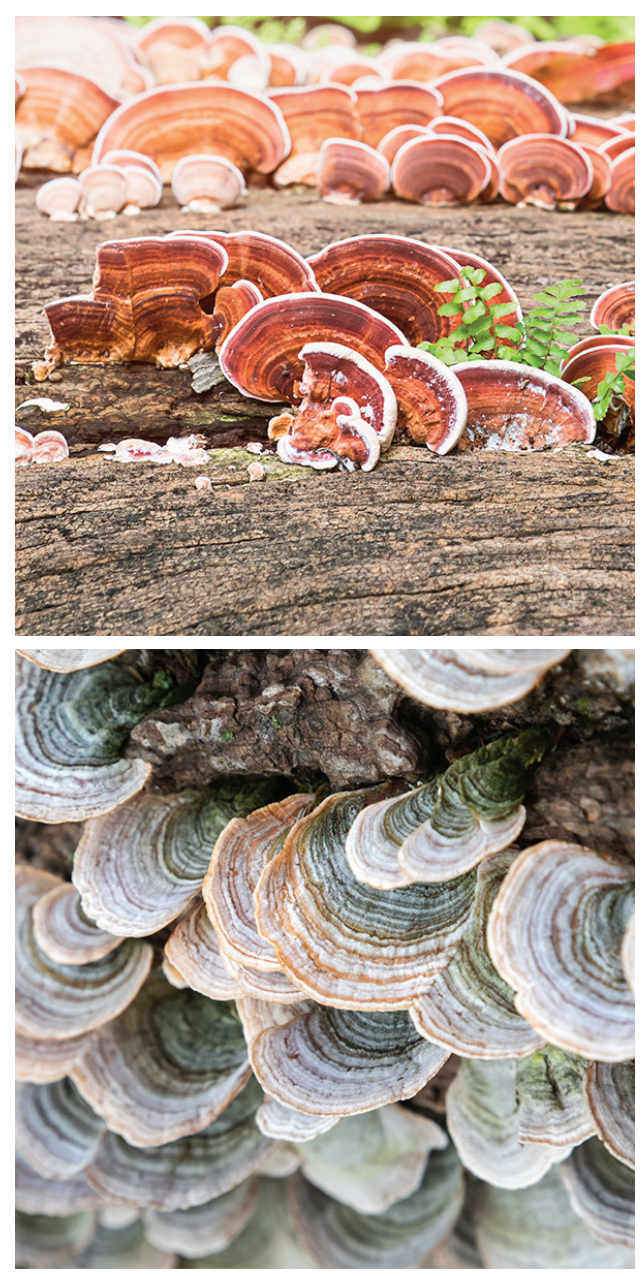

Note: Watson, M. (n.d.). What Are Oyster Mushrooms? The Spruce Eats. (https://www.thespruceeats.com/what-are-oyster-mushrooms-4172003)

Note: Washington, J, Kris, Cynthia, Barbara, Karen, Simard, R. (2019). Medicinal Mushrooms: Powerful Healers or Just Hype? KrisCarr.com.

(https://kriscarr.com/blog/medicinal-mushrooms/) 


\section{NATIVE FUNGI FROM NEW ZEALAND}

The New Zealand Fungal Herbarium at Landcare Research, Auckland, is the centre for scientific research on New Zealand's fungi. It has around 80,000 dried specimens from around the world. A further 7,000 living strains of fungi are held in the adjoining national culture collection. Fungal scientists (mycologists) are currently researching native fungi.

Dr.Peter Buchanan is one of the leading fungal scientists who research on fungal taxonomy, applied uses of wood decay species and fungal conservation. He is also keen to promote awareness and education about fungi, co-establishing the annual NZ Fungal Forays in 1986 and, for more general biodiversity awareness, urban Bio Blitzes from 2004 (Manaaki Whenua-Landcare Research (n.d.).

In discussion with Dr. Buchanan, it was decided that it would be interesting to conduct experiments using native species of fungi from New Zealand. Following his expert advice, two native cultures (ICPM 115546 and ICPM 16414) were included in this research.

\section{FIGURE 3.3}

The Blue Entoloma

(One of Many Native Fungi inNew Zealand)

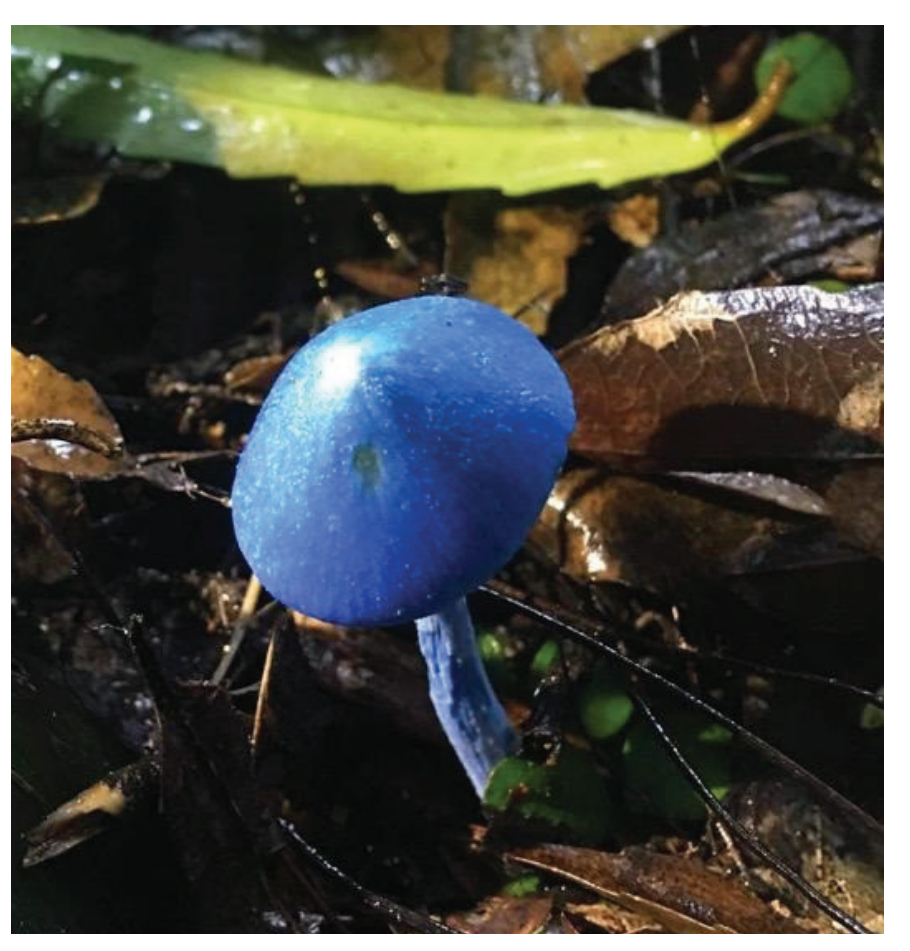

Note: Photograph by the author (2020) 
As native fungi, Flammulina velutipes (Curtis) $P$. Karst. - ICPM 16414 and Pleurotus purpureoolivaceus (G. Stev.) Segedin, P.K. Buchanan \& J.P. Wilkie - ICPM 115546 were used in the growth study of mycelium.

Flammulina is an antitumor substance that is purified from the aqueous extract of basidiomes of Flammulina velutipes (Curt.: Fr.) P.Karst (Wasser).

Pleurotus purpureoolivaceus is a gilled fungus native to Australia and New Zealand. It is found on the deadwood of Nothofagus trees. Although morphologically similar to some other Pleurotus fungi, it is a distinct species incapable of cross-breeding and phylogenetically removed from other species of Pleurotus (Segedin et al., 1995).

Culture samples of the above two species received from ICPM New Zealand were inoculated into growing mediums, following different techniques to grow mycelium.

\section{FIGURE 3.4}

Culture Samples of Native Fungi Received from ICPM New Zealand

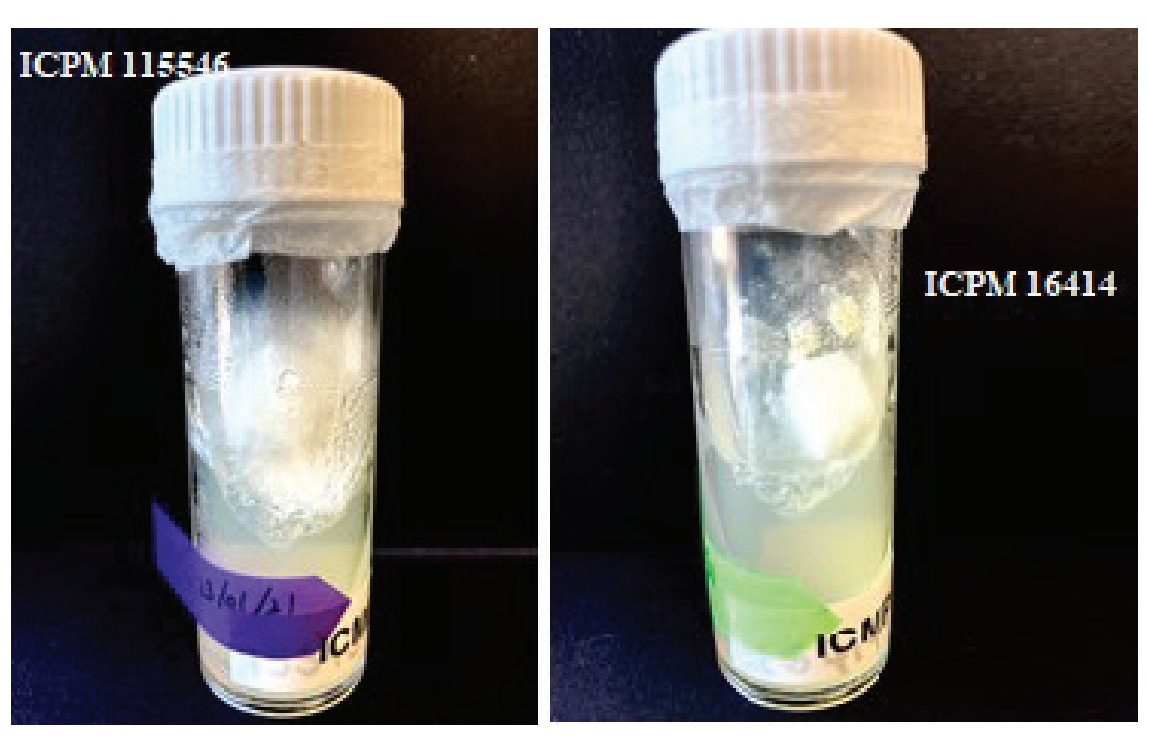

Note: Photograph by the author (2020) 


\section{COMMON PRACTICES IN GROWING MYCELIUM}

Mycelium-based materials are grown using several methods that exploit its ability to interlock with other substances within its network to form a bulk material, harvesting a liquid culture of mycelium (Karana et al., 2018) using inoculation techniques on growing mediums such as Petri dishes.

The first method follows a technique of inoculating an individual strain of mycelium fungus in a substrate consisting of organic substances such as coffee grounds, wood sawdust, rice straw, and cotton fibres. According to Jones et al. (2017), these substrates provide the necessary nutrients for the mycelium to grow, such as carbon (e.g., glucose or fructose), nitrogen, minerals, and vitamins. The addition of the right amounts of water into the substrate is also described as a key factor in successful mycelium growth (Carlile et al.1994).

Commercial and domestic mushroom growers usually cultivate their products using this method. Mushroom spores sourced naturally or purchased as commercial growing packs are used to generate mycelium colonies by inoculating selected substrates. Depending on the mushroom variant, the types of substrates need to be varied. Some aggressive species like oyster mushrooms will grow on coffee grounds, while others require wood to digest for energy (Sayner, 2021). This is generally done under sterile conditions to minimise the chances of mould or other fungi species. Once this inoculation process is done, the substrates are stored in a warm and dark location, for the incubation process to take place. Mushroom growth is visible when the process advances beyond the excrescence of mycelium. Researchers who use this method obtain well-grown mycelium colonies by curbing the growth at the correct phase, which takes from a few weeks to a few months, depending on the variant.

Another method used in laboratories is through pure mycelium materials obtained from a liquid culture of mycelium are placed in static or machine-shaken containers until the liquid fermentation of fungal micro-organisms takes place. When grown in a static liquid culture, filamentous fungi form a mat of hyphae at the surface of the liquid and this mat of hyphae can be dried and used as alternatives for leather, paper, or plastic (Karana et al., 2018). 
Similarly, different forms of mycelium spores are inoculated into pre-made Petri dishes under controlled laboratory conditions. These Petri dishes are then sealed using Micropore tape and stored in dark chambers until the incubation process is completed.

Carlile et al. (1994) emphasised that in any of the growing methods, the mycelium culture should be maintained at controlled environmental conditions of light, temperature, and moisture to ensure stable growth within two to three weeks. Despite the optimal temperature and moisture conditions varying depending on the type of mycelium used, Jones et al. (2017) stated that most species would grow well in a temperature range from 25 to $35^{\circ} \mathrm{C}$. The humidity levels of the environment should be maintained at around 60 to 65 percent to prevent the substrate from drying out (Carlile et al., 1994). According to Deacon (1980), storing the substrates in darkness during the incubation period not only delays the formation of mushrooms but also increases the rapid growth of mycelium.

Through this study of mycelium growing methods, a customised experimental method consisting of all the above growing techniques was designed and developed. 


\title{
EXPERIMENTS CONDUCTED USING THE MATERIAL DRIVEN DESIGN METHOD (MMD)
}

For this stage of the study, three basic steps for producing mycelium were used: activation, fabrication, and desiccation (Grow.bio, n.d).

\author{
Activation - The stage where mycelium growth begins \\ Fabrication - Turning the growth form into desired shapes, products, or designs \\ Desiccation - The baking process of the finished product using an oven or a domestic fan
}

This three-step approach was adopted in structuring the research experimental sequence. Being a series of non-scientific experiments, some of the laboratory condition criteria stated in the literature could not be met. However, in most instances, best efforts were made to adhere to the ideal laboratory conditions using the available resources.

The first phase of the research was to conduct a mycelium growth study, done in two stages as discussed below.

Stage 1 - Growing mycelium using different mediums

Stage 2 - Transforming of grown mycelium into another medium for further growth 


\section{PHASE 1 - MYCELIUM GROWTH STUDY}

In the first stage of Phase 1, the excrescence of mycelium variants was observed through several growing techniques, where they were allowed to grow in different mediums and on different substrates.

FIGURE 3.5

Liquid Culture prepared Using ICPM 115546

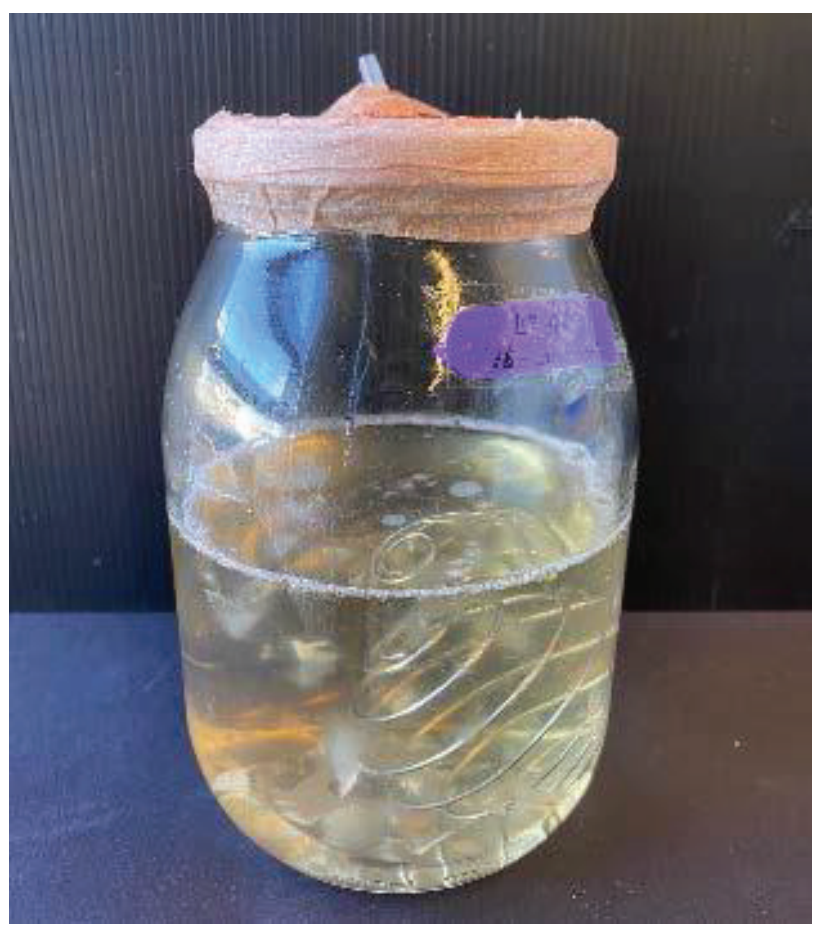

Note: Photograph by the author (2021) 


\section{STAGE 1 - FIRST GROWING TECHNIQUE}

\section{Growing medium: Liquid culture (LC)}

Types of mycelium: Grey oysters, ganoderma, turkey tail, ICPM 16414, ICPM 115546

Method: Three glass jars and two glass tubes were sterilised using the microwave oven. Fifty $\mathrm{ml}$ of LC were poured into each glass jar and $20 \mathrm{ml}$ of liquid culture was put into the glass tubes. Culture samples from each of the five mycelium variants were added into the LC medium. Lids were sealed using Micropore ape and the samples were kept inside a dark chamber for two weeks.

Observations: Mycelium from all five mushroom types had grown in their liquid culture mediums at the end of the experimental period. However, the growth rate of ICPM variants was significantly higher compared to the growth rate of commonly available types.

The liquid culture itself could not be used directly in 3D printing because of its aqueous nature. A more viscous medium was used as the second growing technique, which is potato starch agar (PSA). The experimental sequence using PSA is described as follows. 


\section{SPECIFIC PROCEDURE FOLLOWED WHEN HANDLING CULTURE SAMPLES CONTAINING MYCELIUM}

- $\quad$ All equipment and tools were sprayed and wiped using 90 percent concentrated isopropyl alcohol.

- $\quad$ Sterilisation is a must. Throughout these experiments, this was performed through heating at $120^{\circ} \mathrm{C}$ in a microwave oven for 15 minutes.

- All lids and covers were sealed using Micropore tape to ensure no contamination through the air.

- Once inoculated with mycelium, samples were kept in a dark enclosed space, enabling the incubation of mycelium

- The temperature inside the dark chamber was maintained at around $24^{\circ} \mathrm{C}$.

- A period of 12 to 14 days was needed for the mycelium to complete its colonisation.

- $\quad$ Containers were shaken well daily to dissolve any precipitants. 
FIGURE 3.6

Mycelium Growth in Liquid Culture

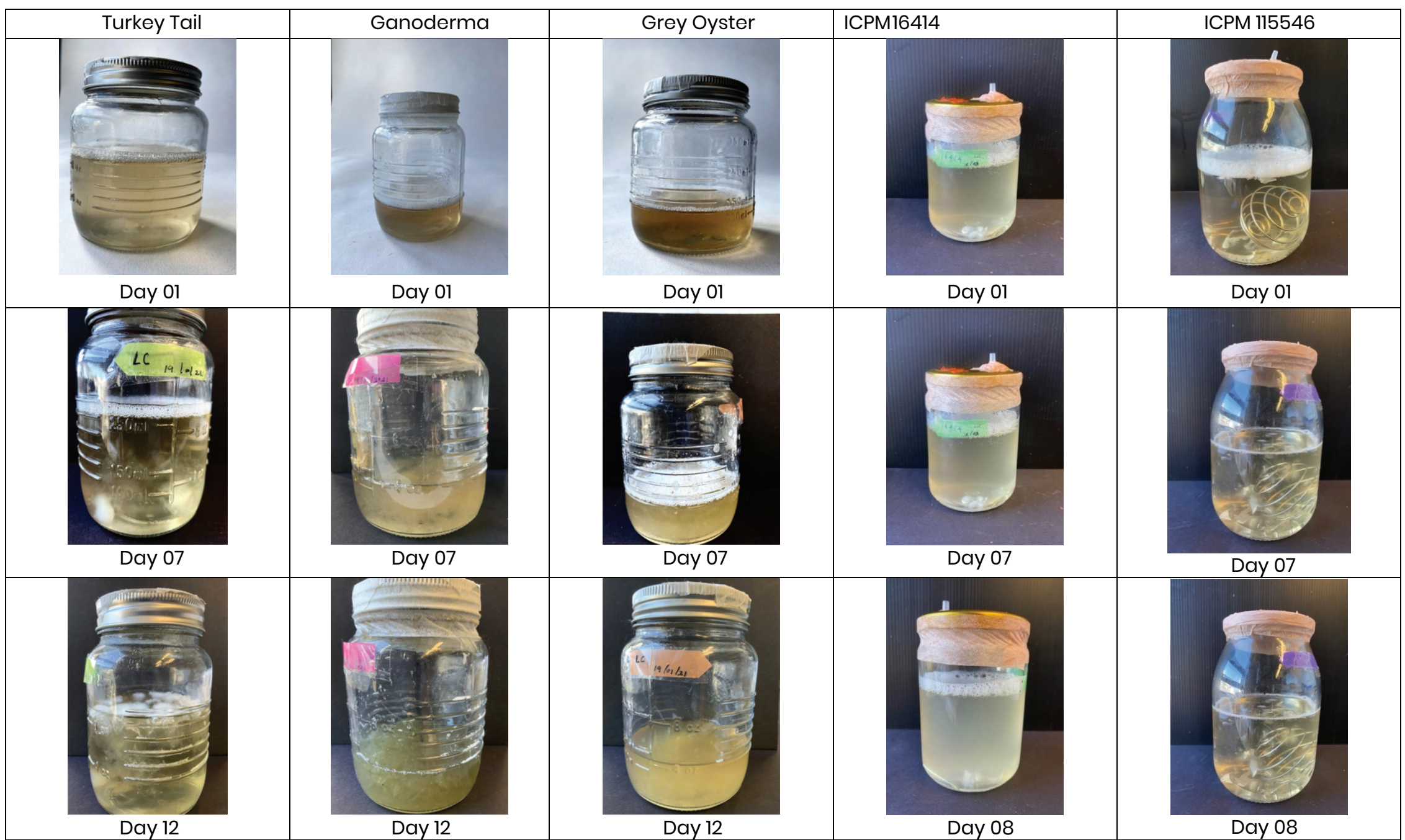

Note: Author's work (2021) 


\section{STAGE 1 - SECOND GROWING TECHNIQUE}

Growing medium: Potato starch agar

Types of mycelium: Grey oysters, ganoderma, turkey tail, ICPM 16414, ICPM 115546

Method: Petri dishes containing potato starch agar were used to observe the mycelium growth of all five mushroom variants. Ganoderma applanates, agar samples of turkey tail mushrooms, liquid culture samples from grey-oysters, ICPM 16414 and ICPM 115546 were added to the Petri dishes, sealed and kept in the dark to observe the mycelium growth.

FIGURE 3.7

Mycelium Growth in Potato Starch Agar

Note: Author's work (2021)

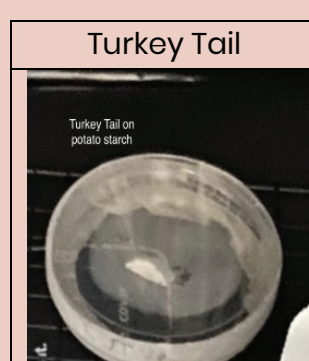

Day 01

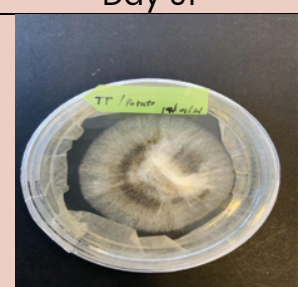

Day 07

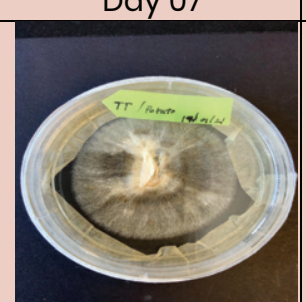

Day 14

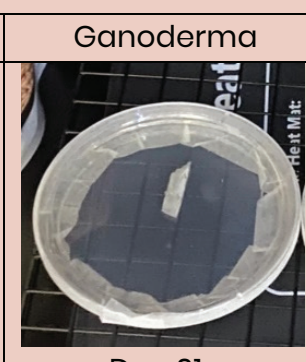

Day 01

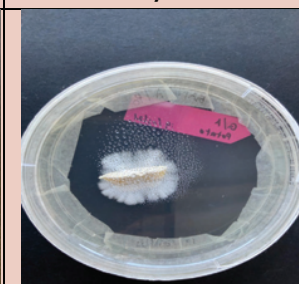

Day 07

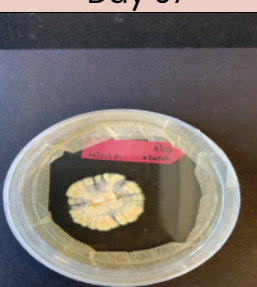

Day 14

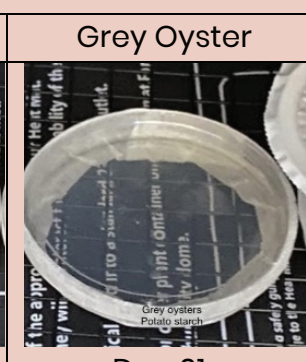

Day 01

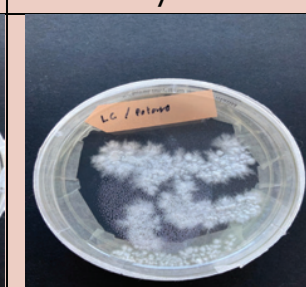

Day 07

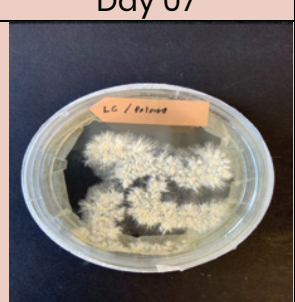

Day 14
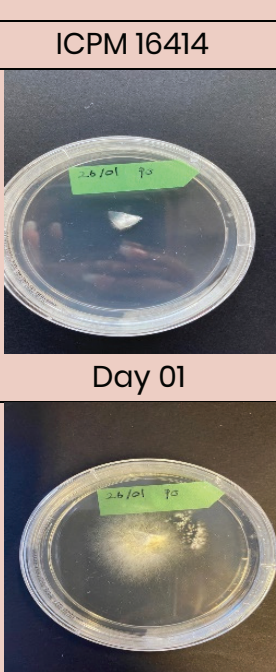

Day 07

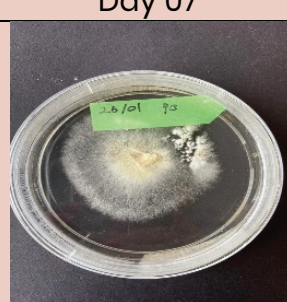

Day 14
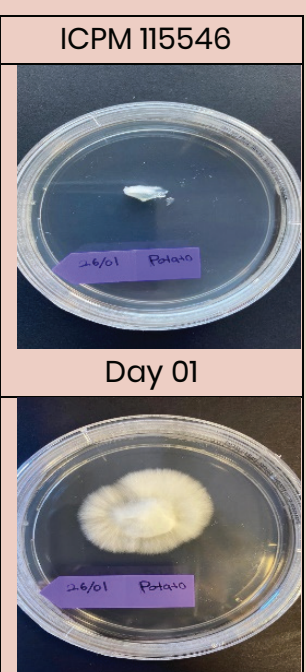

Day 07

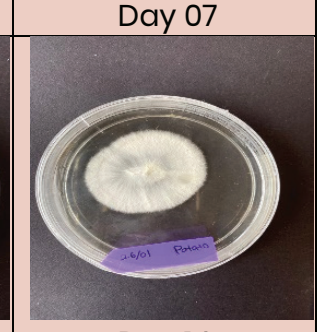

Day 14 
Observations: Successful growth of mycelium could be observed in all five variants. The growth rate in PSA was much higher than in LC medium.

Another starchy medium, but of a more solid nature compared to PSA, was tested to check how well the mycelium could grow. The "Uncle Ben's Tek" technique (StevenBigShroom, 2020) was adopted as the third growing technique and the procedure is described below.

This is a common technique used by researchers in growing mushrooms for various experiments, under laboratory conditions. Here, the source of nutrition for mycelium growth is whole grain brown rice that is pre-cooked and packeted.

\section{STAGE 1 - THIRD GROWING TECHNIQUE}

Growing medium: Pre-cooked whole grain brown rice

Types of mycelium: Grey oysters, ganoderma, turkey tail

Method: Following the technique described by StevenBigShroom (2020) in his web article on Reddit, cooked bags and cups of whole grain brown rice were used as the growing medium. Two bags were semi-opened and samples from turkey-tail agar and ganoderma applanates were placed inside the cooked rice and re-sealed using Micropore tape. A grey oyster mushroom culture sample was added to a cup of cooked brown rice and resealed. Limited growth was observed; however, further growth could be promoted through mild s queezing of the bags or cup. 
Observations: This method was successful for only two variants. Ganoderma did not show any growth inside the rice bag. Turkey tail and grey oyster variants showed comprehensive growth, with the outcomes of these experiments being used in stage two of the first phase.

Next, the effects on mycelium growth of having solid objects in the growing medium were tested. As the fourth growing technique, solid materials of different plastic and fabric properties were selected and the experiments illustrated in Figure 3.8 were performed.

FIGURE 3.8

Mycelium Growth Under "Uncle Ben's Tek" Technique

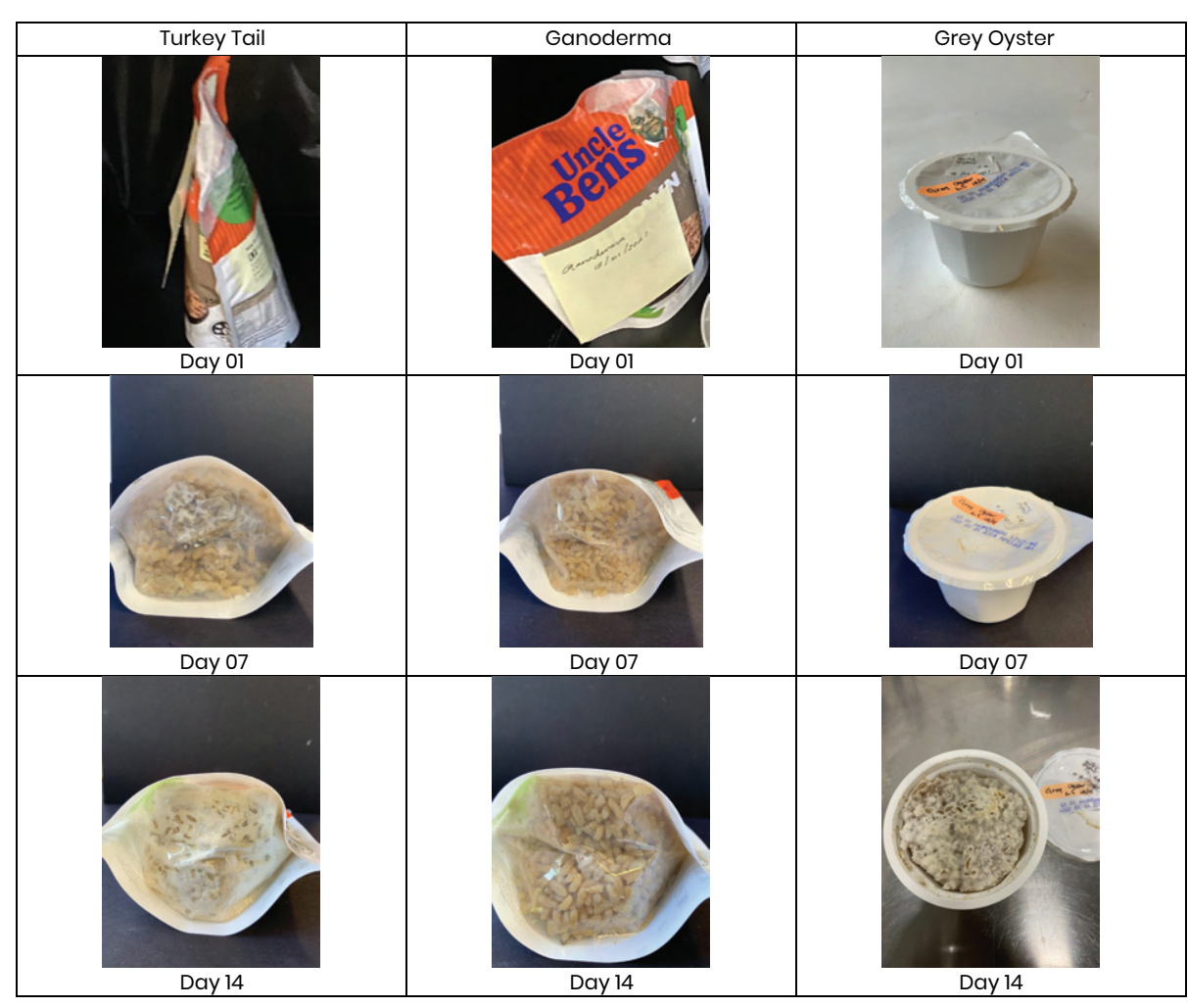

Note: Author's work (2021) 


\section{STAGE 1 - FOURTH GROWING TECHNIQUE}

Growing medium: Materials with different fabric and plastic properties, soaked in liquid culture

Types of mycelium: Grey oyster, ganoderma, turkey tail

Method: Small pieces of 3D printed PLA, wool, cotton crochet, wood straw, and cotton yarn were placed inside glass jars and sterilised using a microwave oven. Thirty-five $\mathrm{ml}$ of liquid culture was added to each jar. Culture samples from the three mycelium types were added separately to them, sealed, and stored in a dark chamber.

\section{FIGURE 3.9}

Growth of mycelium in plastic and fibrous mediums soaked in liquid culture

\begin{tabular}{|c|c|c|}
\hline Growth of Mycelium from Turkey Tail \\
\hline $\begin{array}{c}\text { In cotton wrapped } \\
\text { 3D printed PLA + LC }\end{array}$ & In sterilisedwool + LC & In 3D printed PLA + LC \\
\hline & & \\
\hline Day 07 & & \\
\hline
\end{tabular}




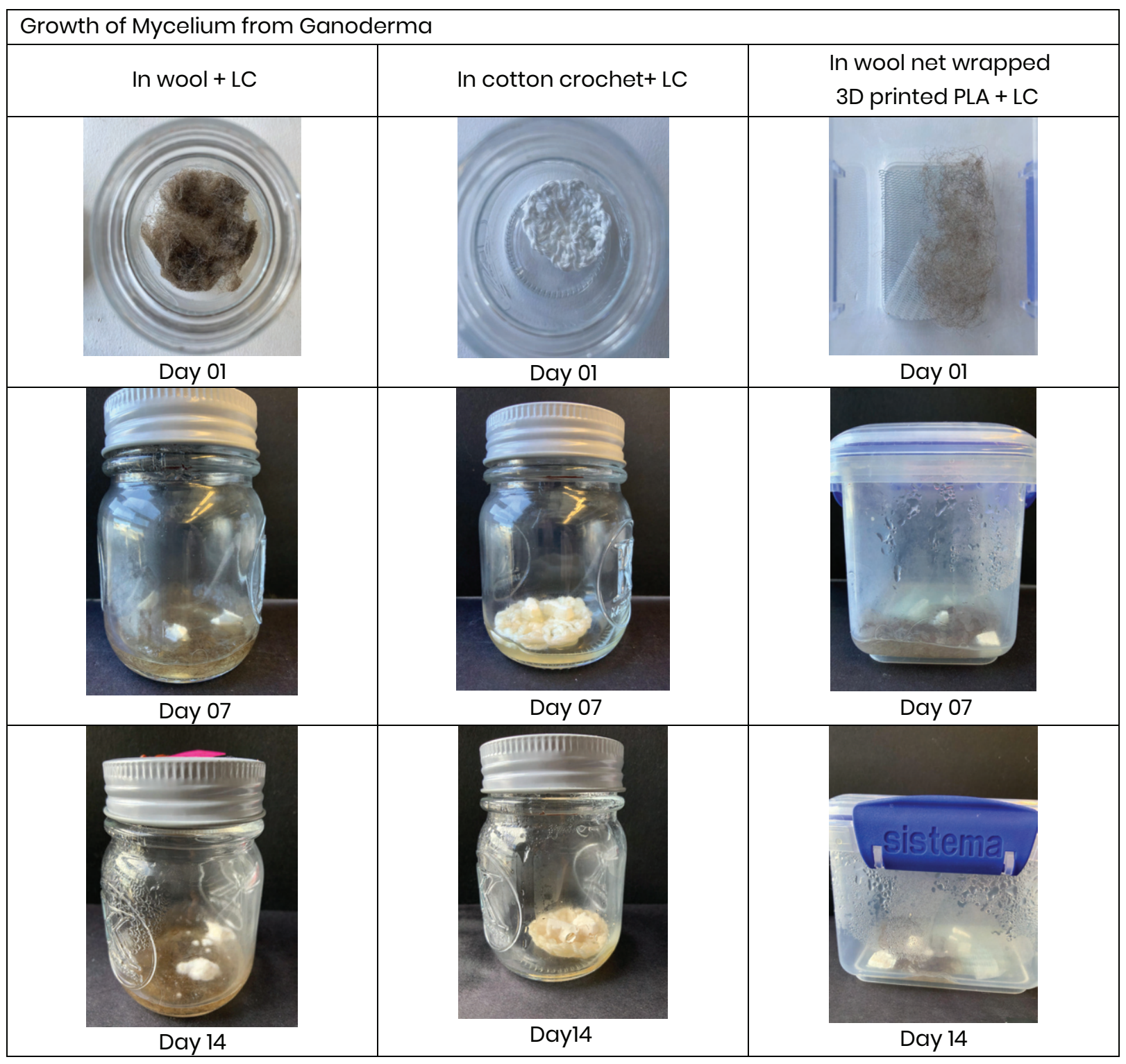




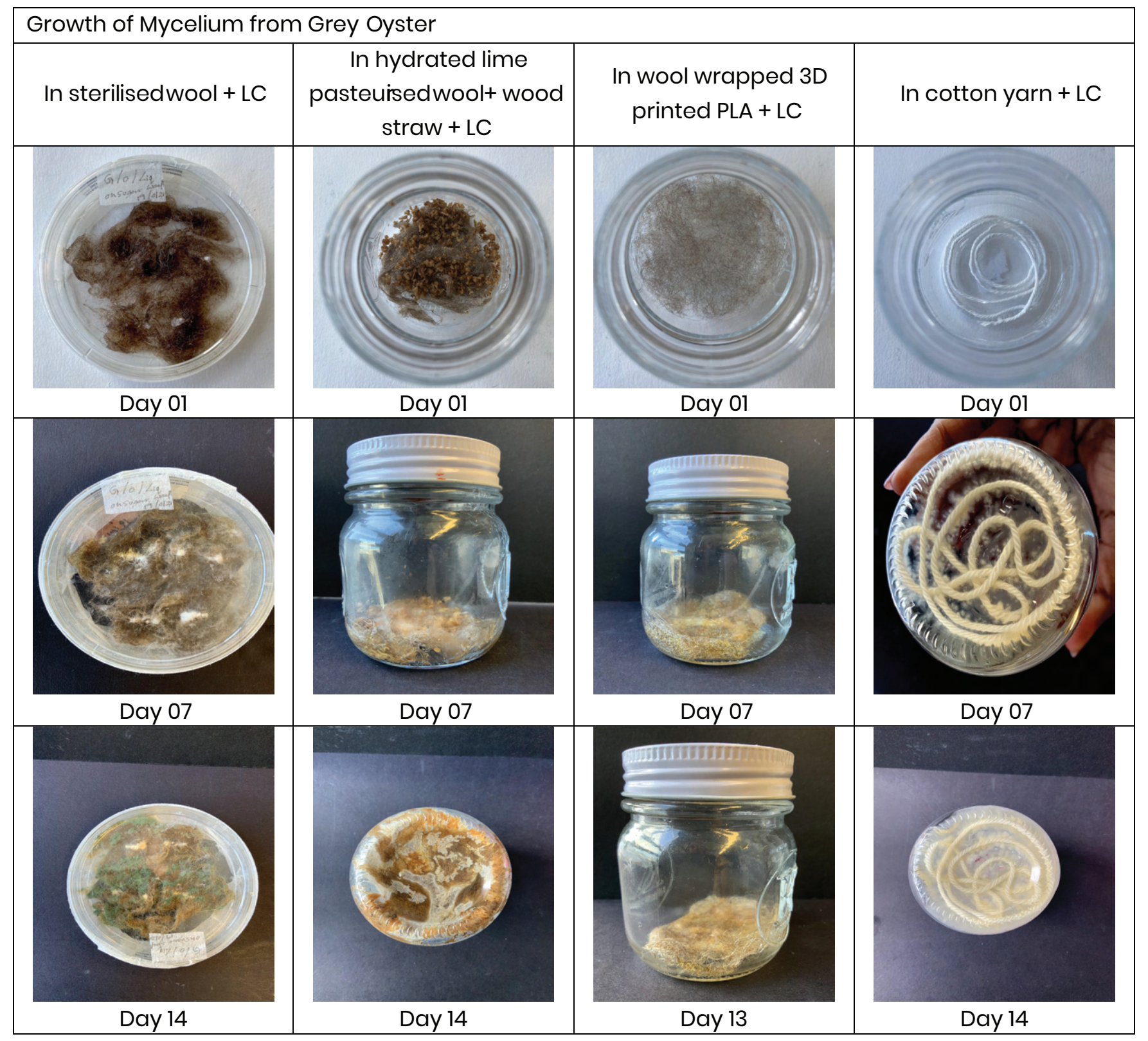

Note: Author's work (2021) 
Observations: All mycelium types had grown on their respective substrates. The growth rate in jars with fibrous materials as substrates had increased more than the in the jars of plastic material substrates.

Triggered by the above observations, further experiments were conducted on the nature of mycelium growth on fabric surfaces made by joining many fibrous materials together. Therefore, in the next set of experiments, the natural fabrics jute, dyed jute, muslin, and cotton rug-liner were used as growing substrates. A pre-made potato dextrose agar (PDA) medium was selected as the nutritional source for mycelium growth.

\section{PREPARATION OF POTATO DEXTROSE AGAR (PDA)}

Ingredients:

$\begin{array}{ll}\text { Potato starch powder } & 200 \mathrm{~g} \\ \text { Dextrose } & 20 \mathrm{~g} \\ \text { Agar } & 20 \mathrm{~g} \\ \text { Distilled water } & 11\end{array}$

Steps:

Mix all ingredients and boil in distilled water until they dissolve.

Disinfect the containers using isopropyl alcohol and pour the mixture into them.

Sterilise in the microwave oven at $120^{\circ} \mathrm{C}$ for 15 minutes. 


\section{STAGE 1 - 5TH GROWING TECHNIQUE}

Growing medium: Fabric materials, soaked in PDA

Types of mycelium: Grey oyster, ganoderma, turkey tail

Method: Selected substrate material samples were soaked in PDA, placed in plastic air tight containers, sterilised using a microwave oven, brought back to room temperature, and inoculated with the three types of mycelium liquid cultures. Containers were sealed using Micropore tape and stored in the dark until the incubation period was completed.
FIGURE 3.10

Growth of Mycelium on Fabric Substrates Soaked in PDA

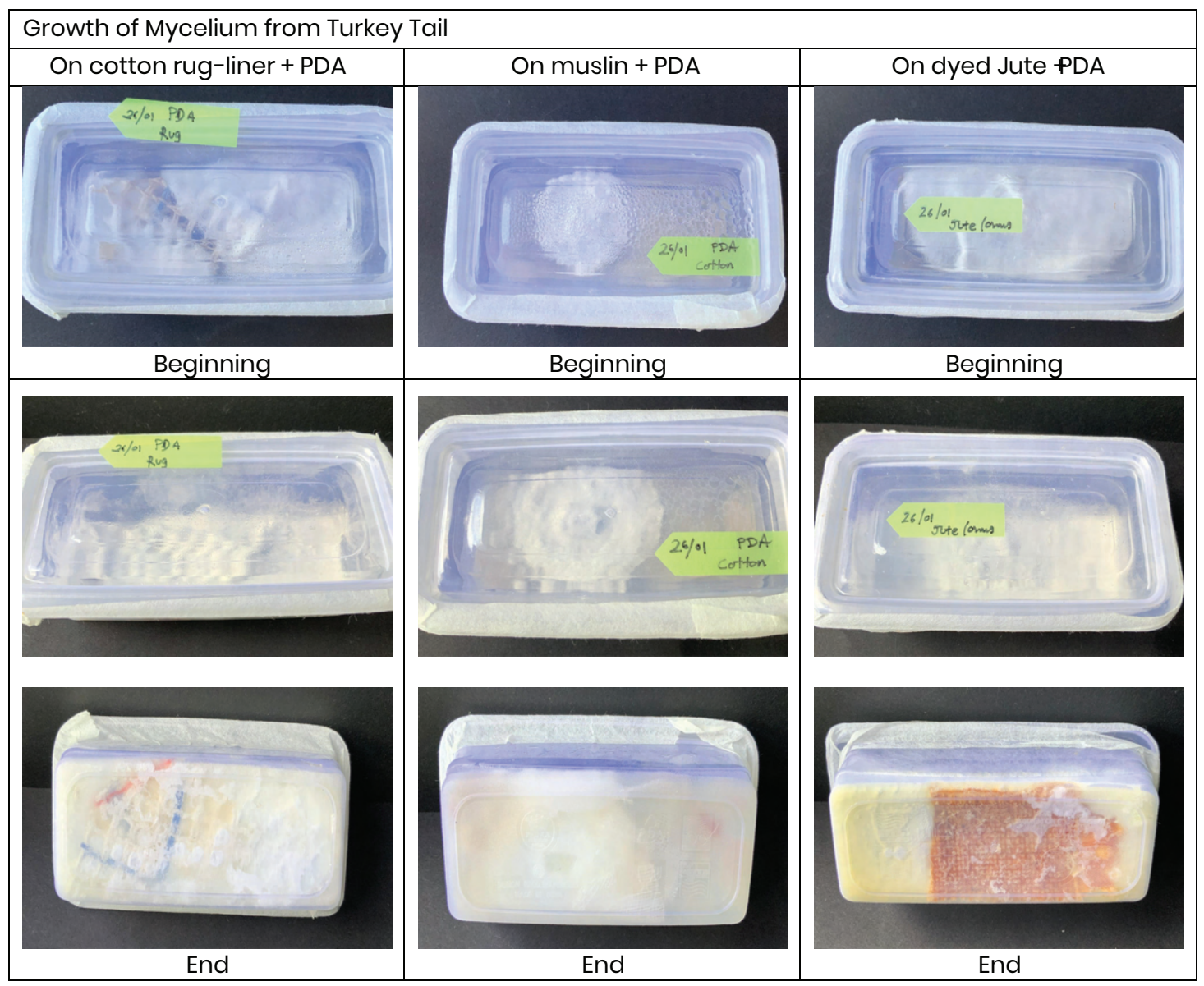




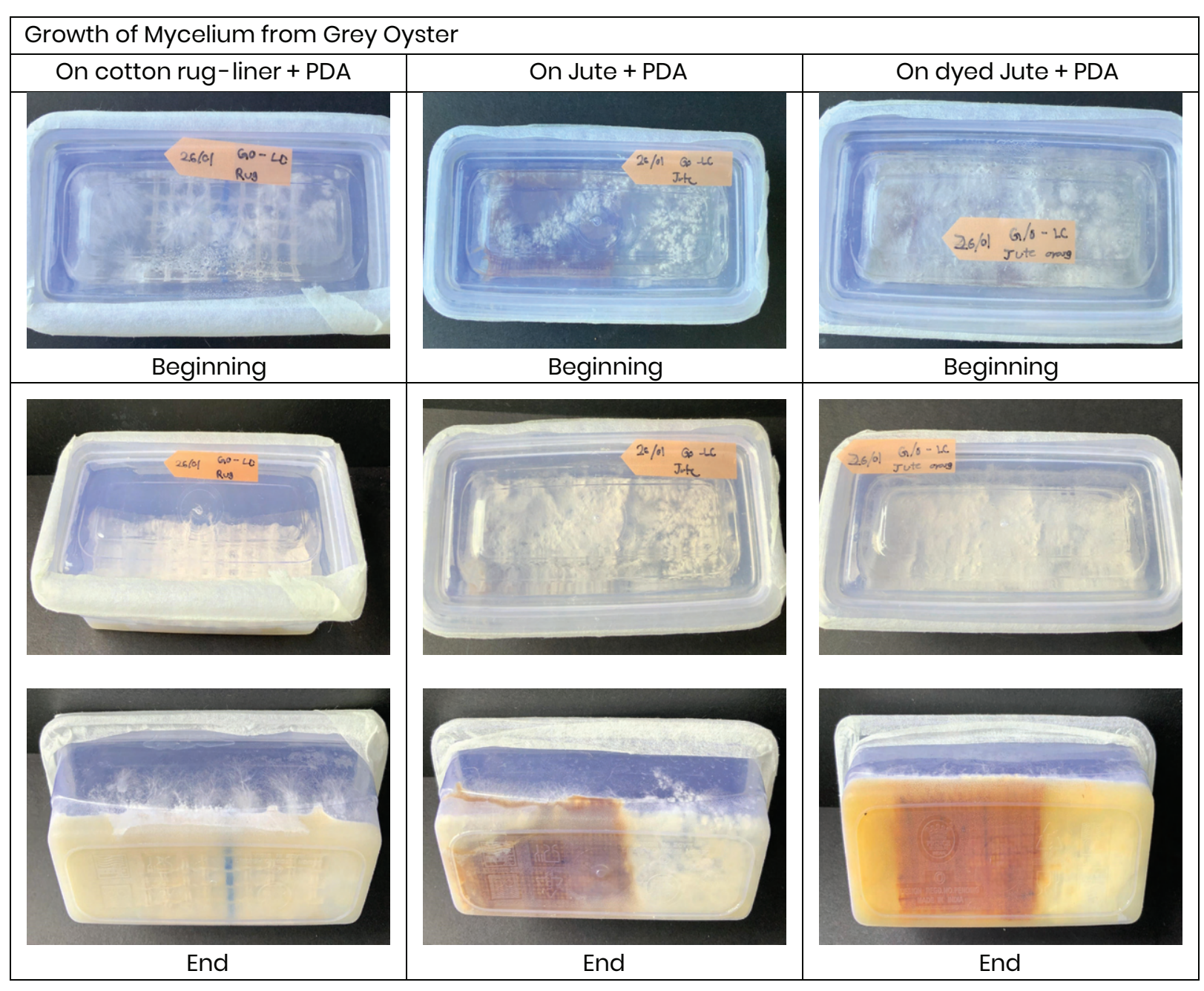




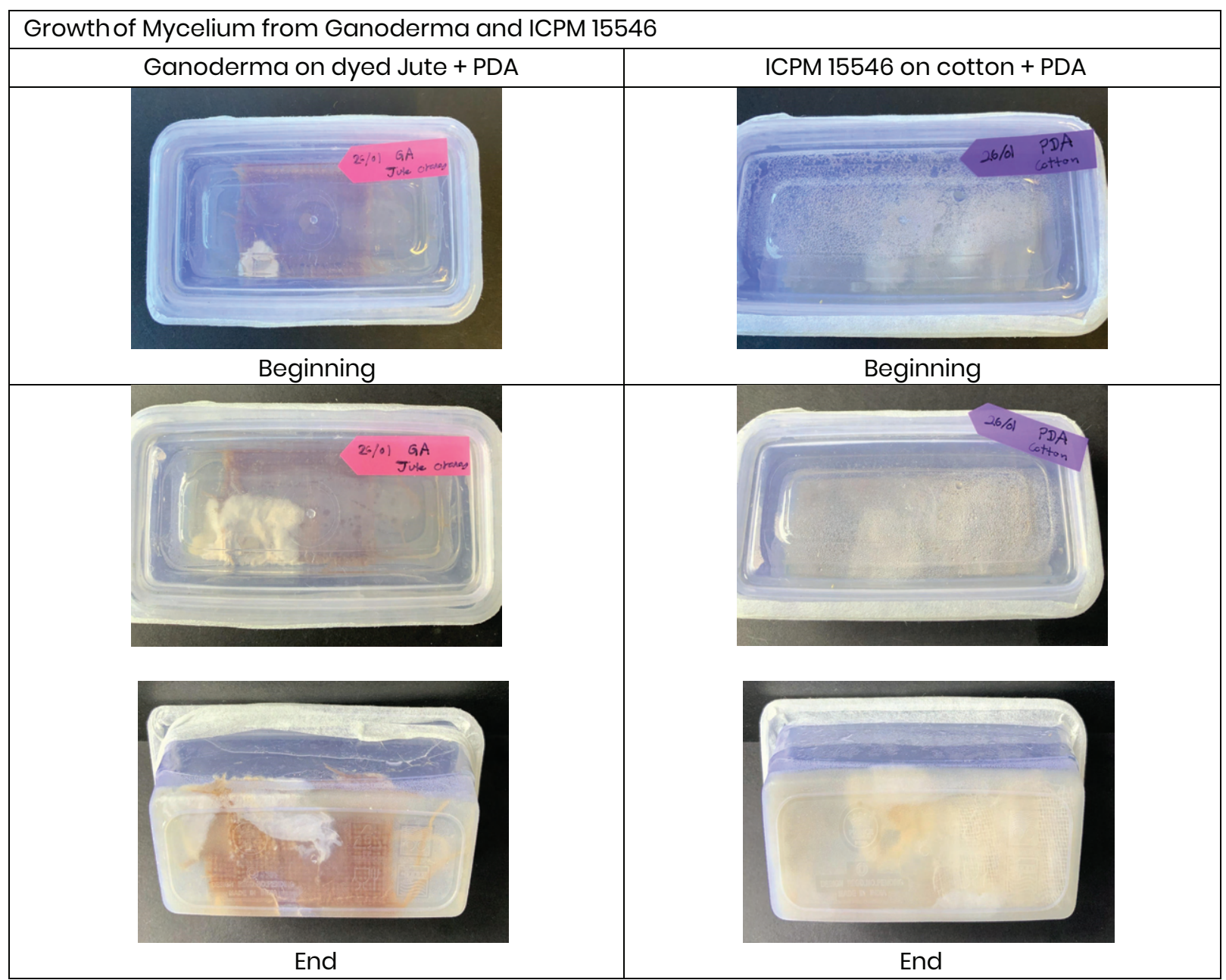

Note: Author's work (2021)

With sound knowledge gathered on how mycelium growth takes place in different substrates, the second stage of experiments was conducted to examine the ability of grown mycelium to transform its excrescence from one medium to another substrate or medium. 


\section{STAGE 2 - FIRST EXPERIMENT}

Origin of mycelium: mushroom pack, where mycelium had already grown in a medium of coffee-wood straw mix with adequate moisture.

Secondary substrate: Plastic wireframe

Method: The ready-to-grow mushroom pack purchased from a local market was crushed by hand and turned into a paste using a kitchen grinder.

A plastic syringe was filled with the paste mixture and the mixture was extruded onto the plastic wireframe inside a plastic still air container. The container was sealed using Micropore tape and kept in a dark chamber for five days and subjected to visual observations.

Figure 3.11

Growth of Mycelium from Coffee-Wood Straw Medium, Upon Extrusion onto a Plastic Wireframe

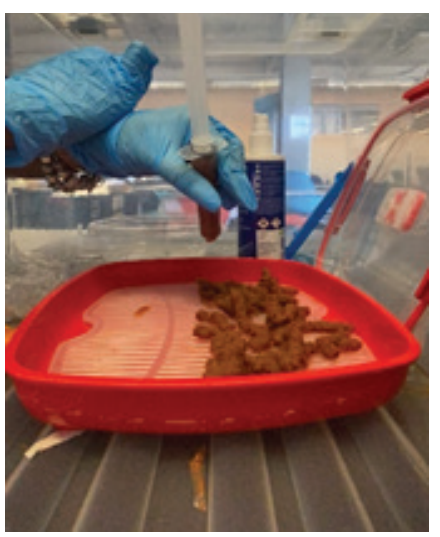

Extrusion of mycelium in coffee-wood straw paste

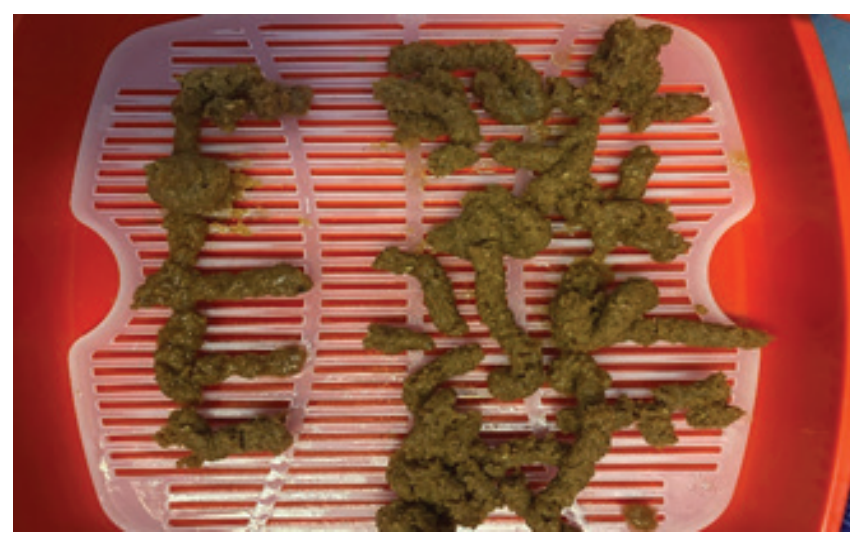

Extruded paste on plastic wireframe

Note: Author's work (2021)

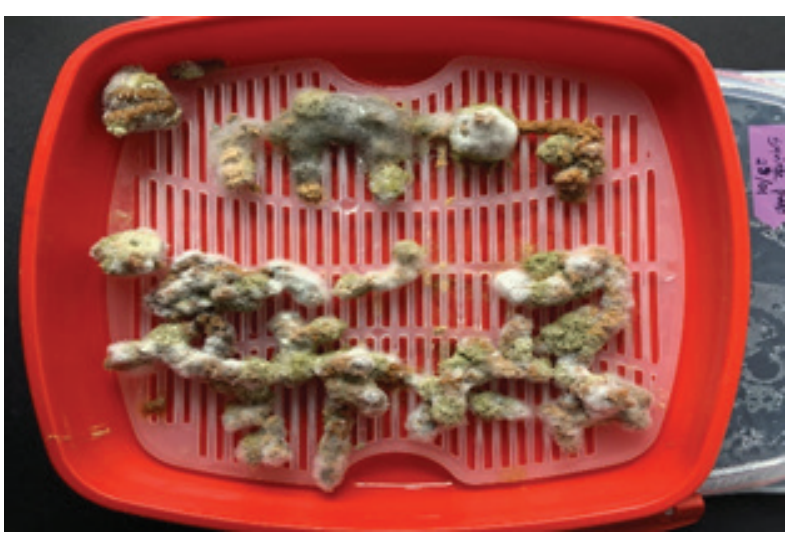

Growth progress on the secondary substrate 
Observations: Despite the mycelium growth being visible, it had become contaminated and dried out.

Although successful mycelium growth was achieved using this technique, considering the weight and moisture factors, the suitability of adopting it into the fashion segments is ambiguous.

In search of alternatives, the second set of experiments were conducted, as illustrated below.

\section{STAGE 2 - SECOND SET OF EXPERIMENTS}

Origin of mycelium: Liquid Culture of ICPM 15546

Secondary substrate: Pulp made of tissue paper towels, mixed with polyvinyl acetate (PVA)

Method: To prepare a more workable paste, tissue paper towels were soaked in water and converted into paper pulp. To make the extruded objects rigid, PVA was added to the pulp, comprising 20 percent of its weight. Two pulp samples were prepared. One was inoculated with ICPM 155546 and extruded onto a plastic sheet using a syringe, placed inside a sealed container and stored in the dark to observe the mycelium growth. The other sample was extruded directly onto plastic sheets in built-up layers, to observe the stability of the extruded paste. 
FIGURE 3.12

Experimental Procedure of Mycelium Growth in Extruded Tissue Paper Pulp and PVA Medium

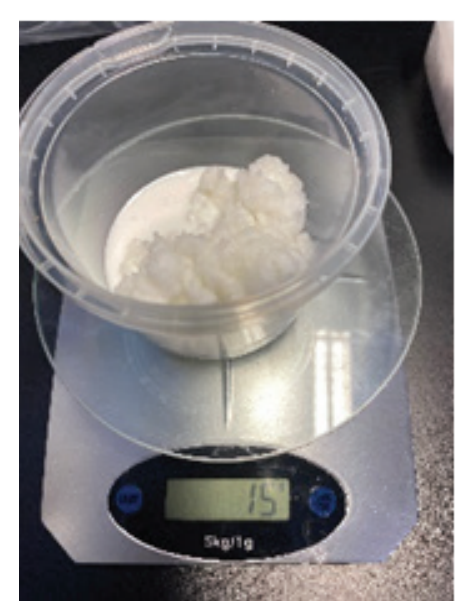

Preparation of paper pulp sample with PVA

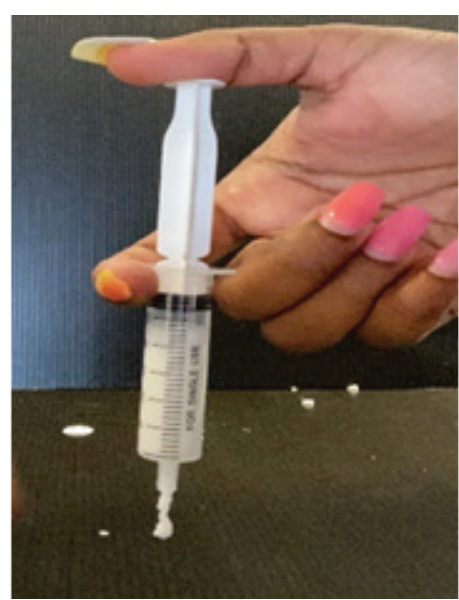

Extrusion using plastic syringe

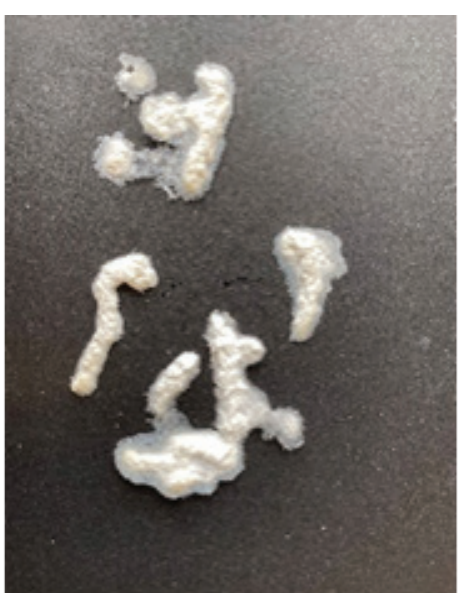

Paper pulp + PVA inoculated with mycelium

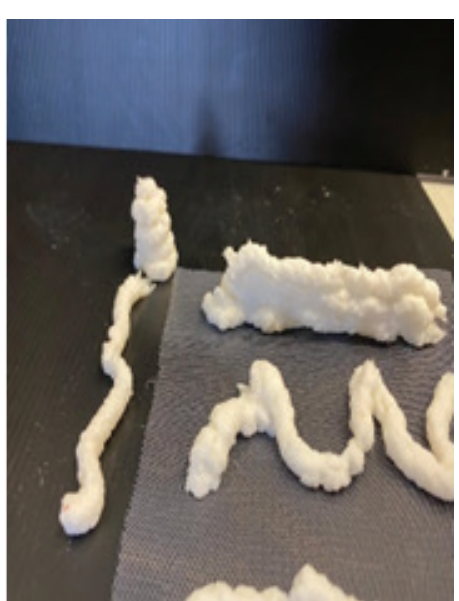

Paper pulp + PVA

Observations: Mycelium growth was visible in the sample inoculated with ICPM 15546. However, two days after extrusion, the paper-PVA paste was fully hardened and there was no continuation of the growth of mycelium beyond this time. The reason was the hardened PVA made an impermeable surface, limiting the mycelium growth. Therefore, it was understood that a mixture of this nature was also not workable due to the constraints for free growth of mycelium after extrusion.

In the third set of experiments, a rice paste prepared using the Uncle Ben's Tek technique, discussed in the first stage of the mycelium growth study phase, was used. The idea was to observe the ability of mycelium to decide its own growth path through different materials. 


\section{STAGE 2 - THIRD SET OF EXPERIMENTS}

Origin of mycelium: Grown mycelium in pre-cooked whole-grain brown rice

Secondary substrate: Two-ply cotton yarn, Petri dish containing potato starch agar

Method: The mycelium-rice mixture was ground together to prepare the paste. A Petri dish containing PDA was used and the rice paste was extruded into the PDA around the internal perimeter of the glass walls.

As another sample, an unground mycelium-rice mixture was placed inside a disinfected plastic container and a piece of two-ply cotton yarn was placed in the same, next to it

Both samples were sealed, sterilised and placed inside a dark chamber allowing the mycelium to incubate.

Observations: Mycelium had grown through the PDA, covering the entire surface of the Petri dish. (as presented in Figure 3.13).

Similarly, the rice mix too was covered by the mycelium growth and had been progressing towards the piece of cotton yarn.
Having bagged these experimental results, the focus was then directed towards developing a more workable extrusion medium with adequate viscous properties, nutrition, and moisture for the growth of mycelium, which could be used directly in the extruder of the robotic arm 3D printer.

With this, the second phase of the research experiments began, designed to develop a semi-liquid culture medium where mycelium could be grown, and perform $3 \mathrm{D}$ printing by using this semi-liquid culture as the extrusion medium. 
FIGURE 3.13

Free Growth of Mycelium in PDA Medium and Through Cotton Yarn

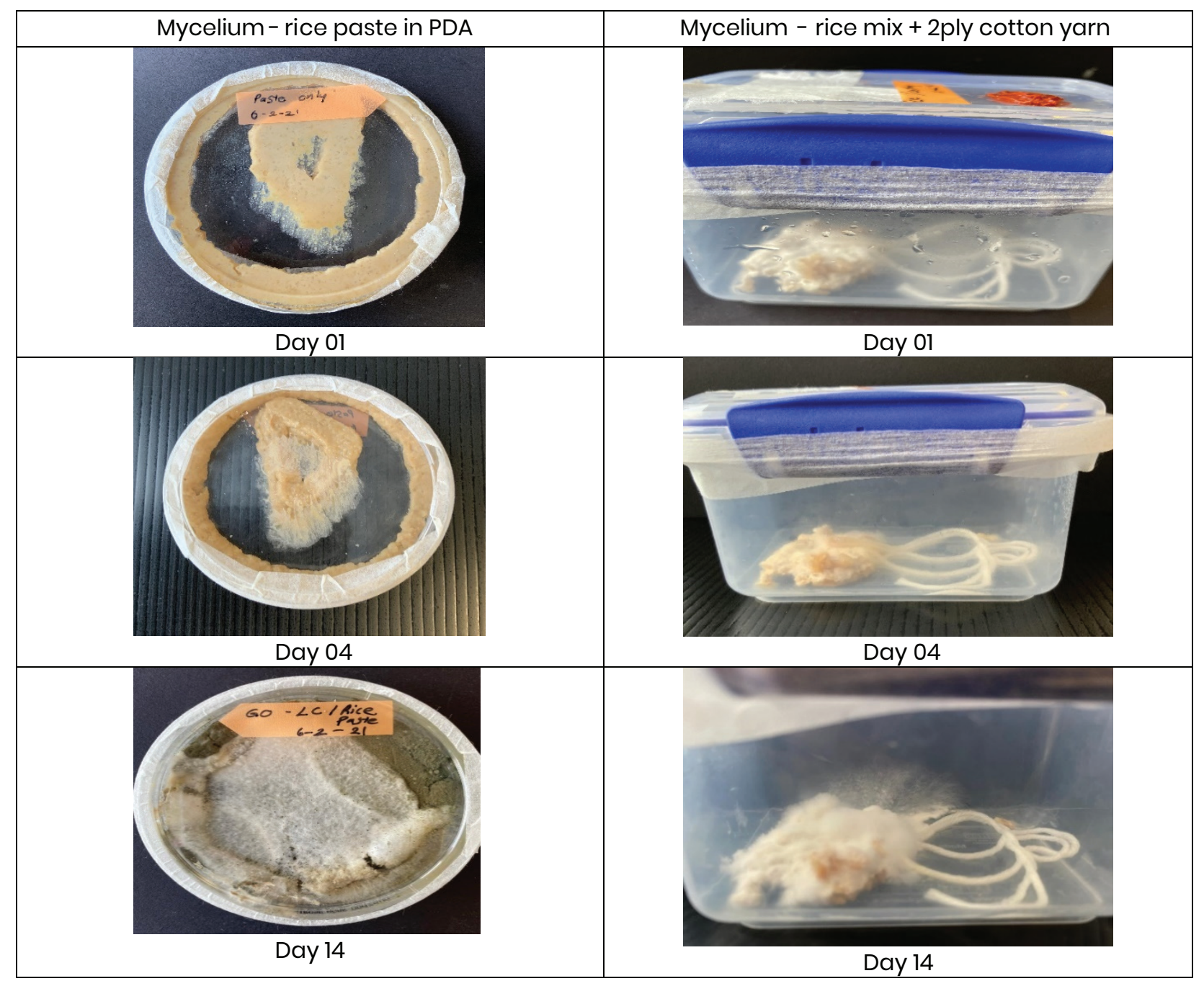

Note: Author's work (2021) 


\section{PHASE 2 - SYRINGE PRINTING EXPERIMENTS AND BAKING}

The second phase of the experimental study consisted of five stages where the outcomes from each stage were used as progressive inputs into the following stages, as shown through the process flow diagram in Figure 3.14.

FIGURE 3.14

Workflow Development of the Experimental Designs Using the Syringe Printing Technique

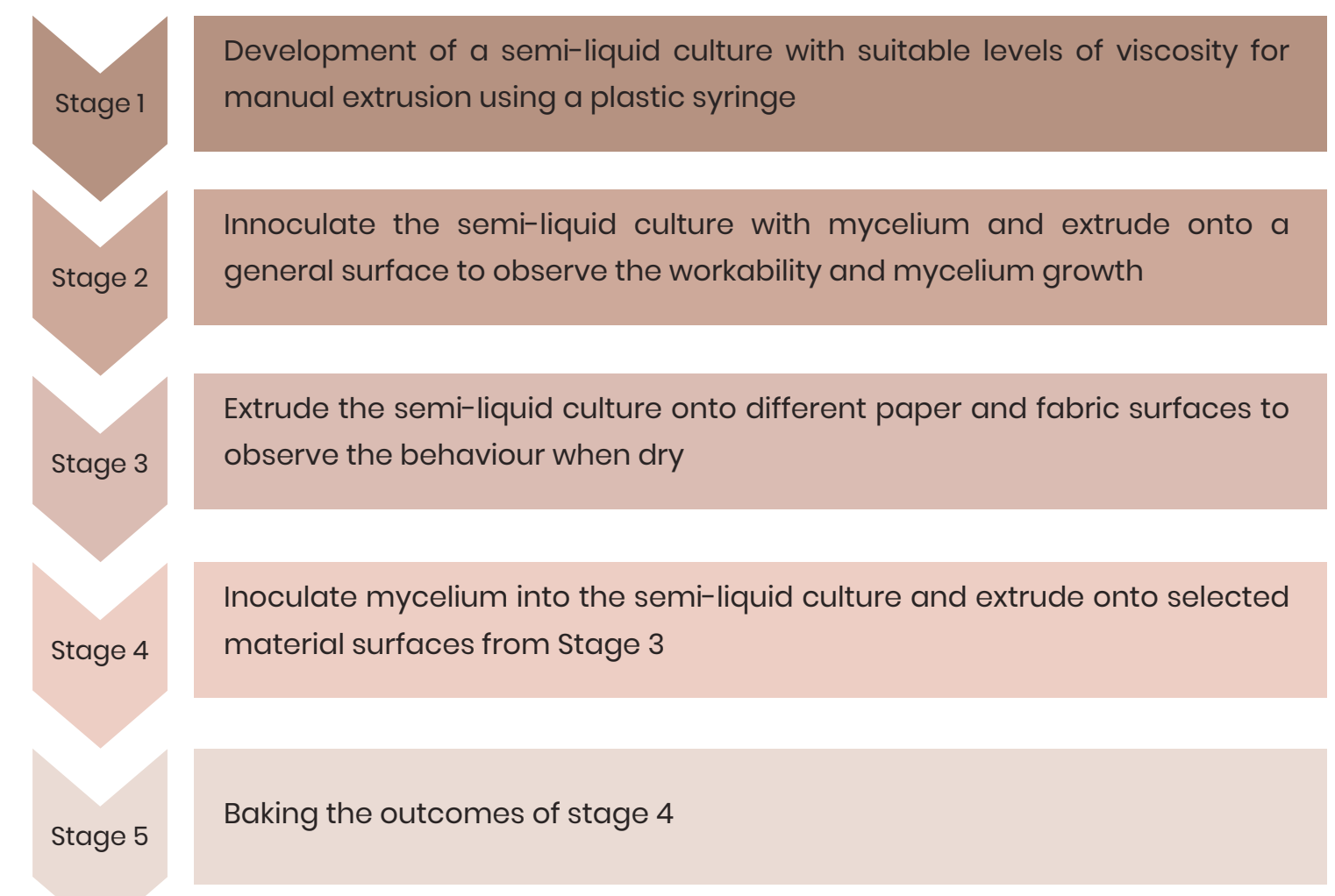

Note: Author's work (2021) 


\section{STAGE 1 - DEVELOPMENT OF A SEMI-LIQUID CULTURE WITH ADEQUATE VISCOUS LEVELS FOR 3D PRINTING}

Several factors were needed to be taken into consideration during the development of this extrusion medium.

- Viscosity - The material should be flowable enough to be extruded through the robotic arm nozzle of a 3D printer. At the same time, it should be hard enough to hold its shape upon extrusion, while not collapsing because of its own weight. Balancing these three factors was a time-consuming exercise that was successfully achieved through visual observations while changing the compositions.

- Nutrition - Nutrition provided by the growing medium is a vital factor for the excrescence of mycelium. Inadequate nutrition levels would lead to lesser growth and the anticipated results might not be obtained.

- Presence of moisture - It was well understood that moisture too, plays a vital role in the successful growth of mycelium in a growing medium. Less moisture would weaken the growth, while excess moisture would result in the growth of mould, leading to contamination. At the same time, the liquidity levels through which moisture is present in the growing medium directly affect the viscosity of the mixture and the drying time. 
Having addressed all the above three factors, a semi-liquid culture medium was developed by mixing the following ingredients in a pan, stirring the mixture while boiling on low/medium heat for 20 minutes and then cooling it back to room temperature, as illustrated in Figure 3.15.

- Potato starch powder - $40 \mathrm{~g}$

- Distilled water-750 ml

- Corn dextrose and dry malt extract mixed in a 4:1 ratio - $20 \mathrm{~g}$

- Agar-agar- $5 \mathrm{~g}$

FIGURE 3.15

Preparation of the Semi-Liquid Culture Medium

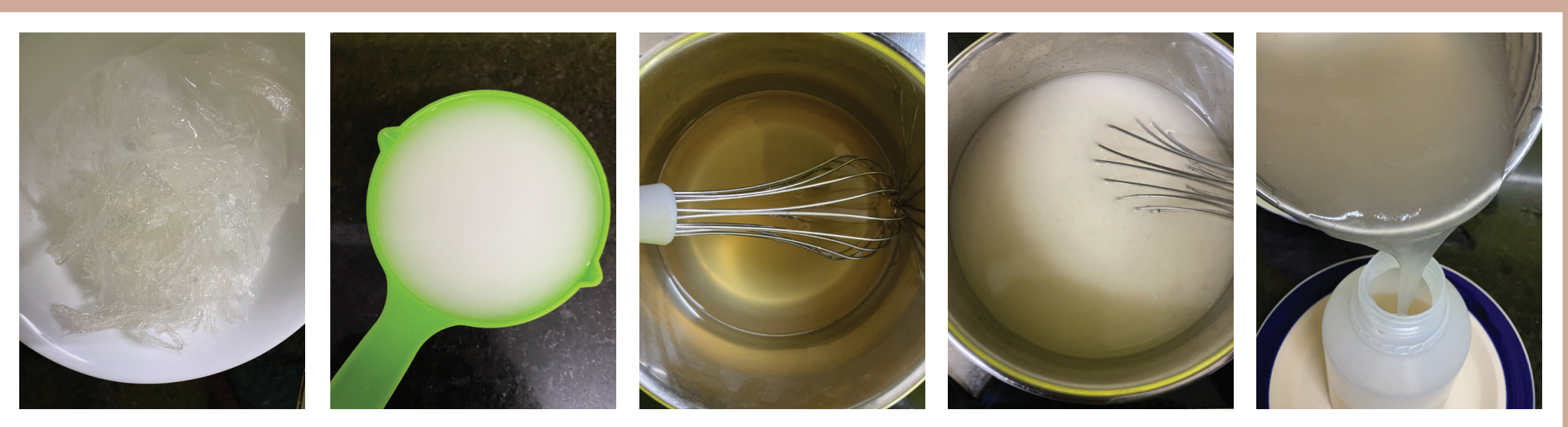

Note: Author's work (2021) 
STAGE 2 - EXTRUSION OF THE SEMI-LIQUID CULTURE WITH MYCELIUM

The behaviour of the mycelium inoculated semi-liquid culture upon extrusion needed to be observed.

First, the prepared semi-liquid culture was inoculated with the liquid culture of the selected native variant of mycelium, ICPM 115546.

Two plastic containers were sterilised and the prepared medium was extruded onto the containers, forming two different shapes using a manual plastic syringe. (Figure 3.16)
FIGURE 3.16

Inoculation and Extrusion Process of the Semi-Liquid Culture

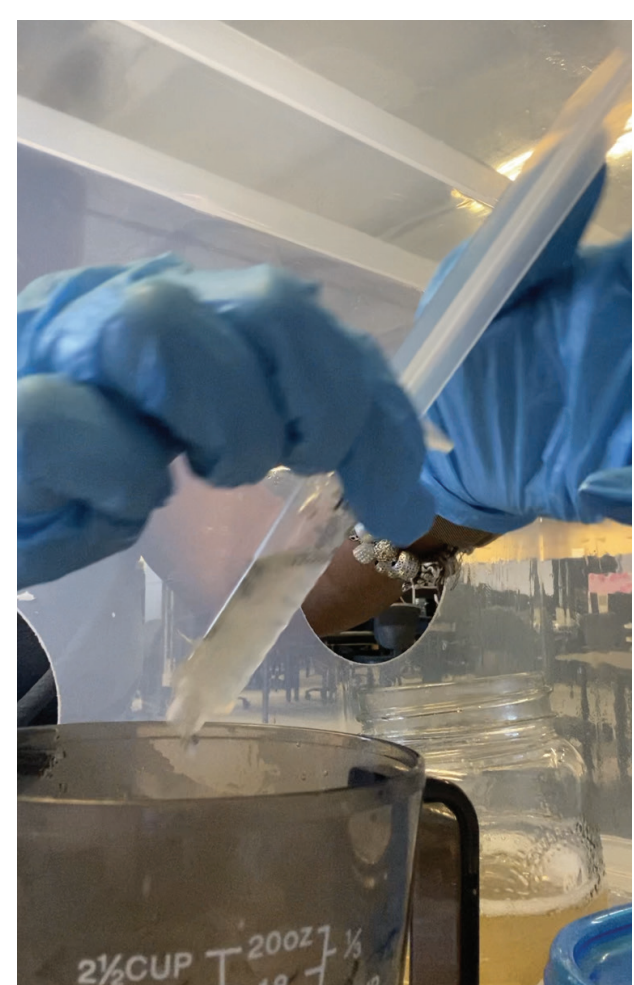

During Inoculation

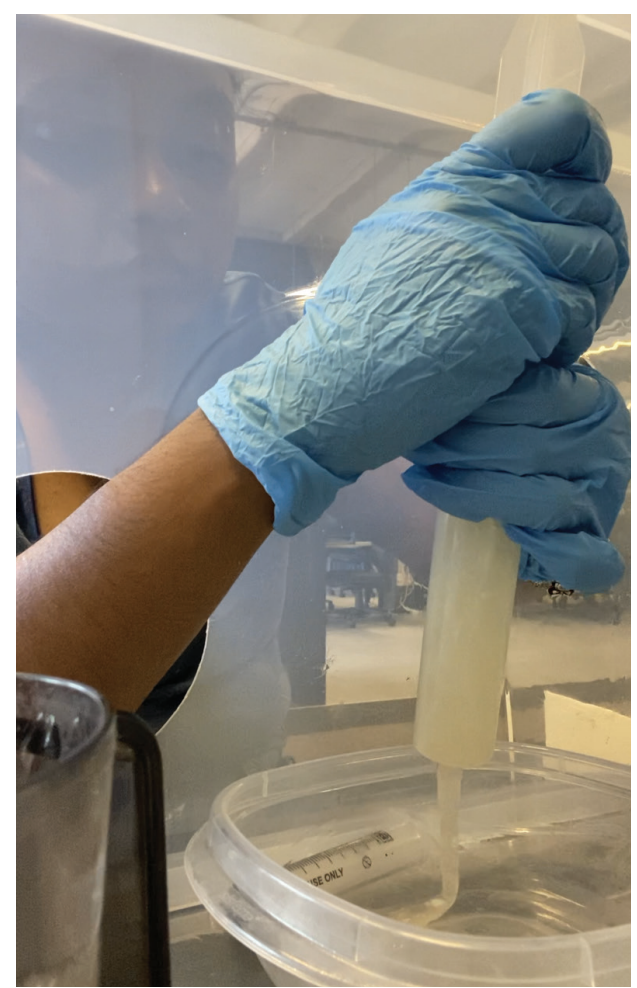

During Extrusion

Note: Author's work (2021) 
Lids were sealed and stored in a dark chamber for two weeks to observe the changes and the growth of mycelium.

Observations made by the author within two weeks from the date of extrusion are shown in Figure 3.17.

As anticipated, it was observed that the semi-liquid culture had performed well and at the same time, mycelium growth was clearly visible.

Therefore, it was understood that the developed semi-liquid culture with the current material composition was a workable mixture for syringe printing and it contained adequate nutritional and moisture levels to support sufficient growth of mycelium over time.

Moving further, extrusion surfaces were varied to investigate different design opportunities that are discussed in the third stage of experiments.

\section{FIGURE 3.17}

Outcomes Within Two Weeks of the Extrusion of Semi-Liquid Culture With Mycelium

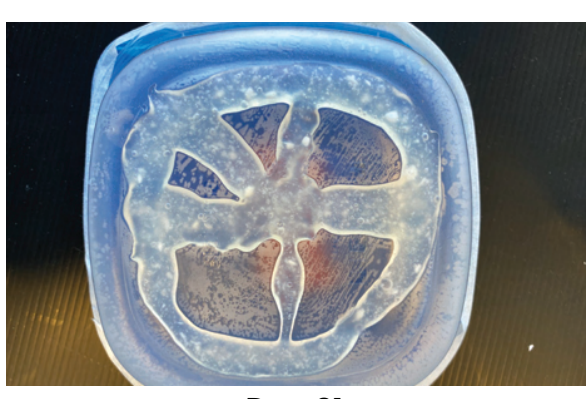

Day 01

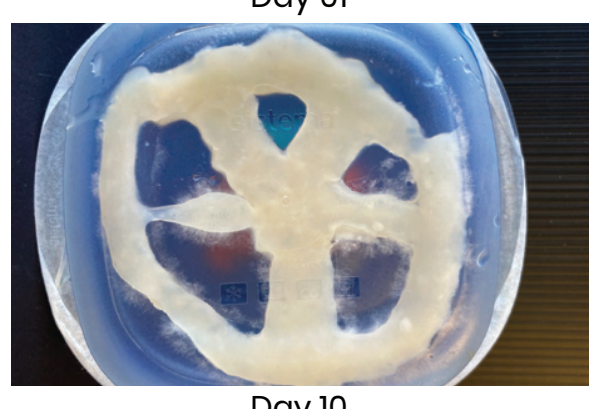

Day 10

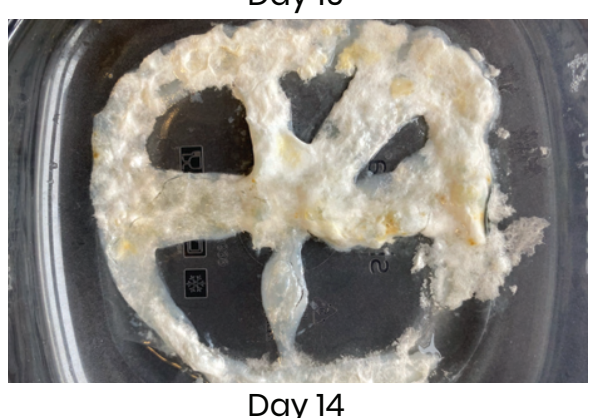

Day 14

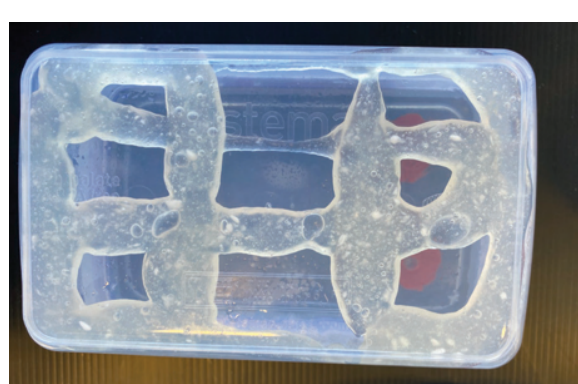

Day 01

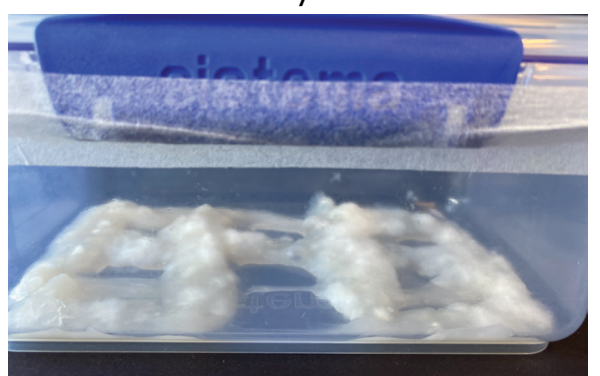

Day 10

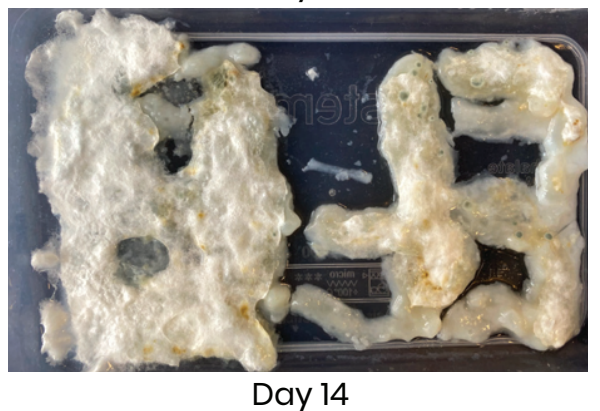

Day 14

Note: Author's work (2021) 


\section{STAGE 3 - EXTRUSION OF THE SEMI-LIQUID CULTURE ONTO FABRIC AND PAPER SUBSTRATES}

The objective was to understand how the developed semi-liquid culture would behave once extruded onto paper and fabric surfaces of different properties and allowed to dry. Three types of fabrics and four types of paper were selected, and the semi-liquid culture was extruded onto them and allowed to dry for two weeks.

\section{Paper types:}

Baking paper

Aluminium foil

Tissue paper

Plastic cling wrap

Fabric types:

Cotton - Formed by natural fibres in high density Muslin - Formed by natural fibres in lower density Georgette - Formed by synthetic fibres

Figure 3.18 below presents the images taken on each surface upon extrusion and seven days after allowing them to dry.

\section{FIGURE 3.18}

Behaviour of the semi-liquid culture on fabric and paper surfaces

\section{Baking Paper}

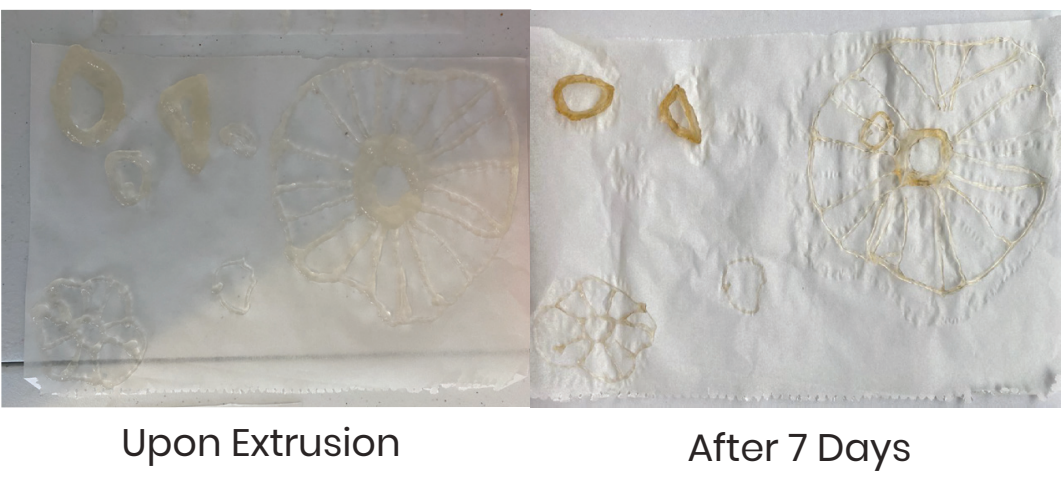

\section{Tissue Paper}

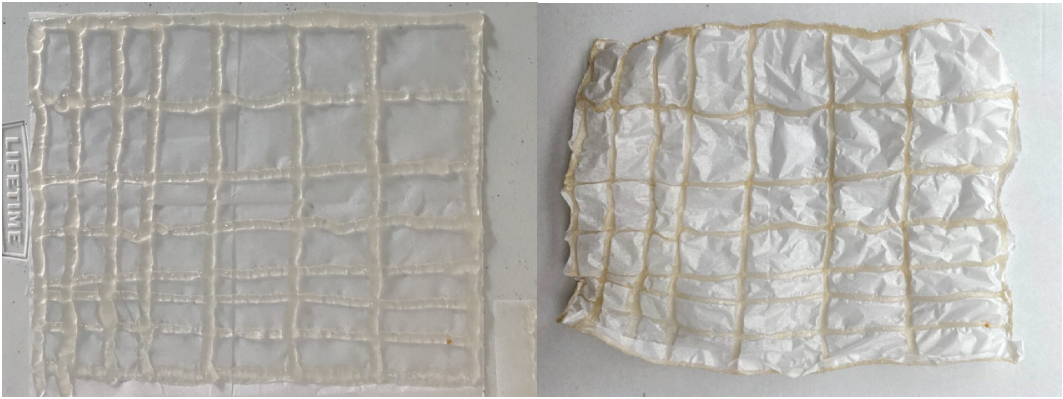

Upon Extrusion 


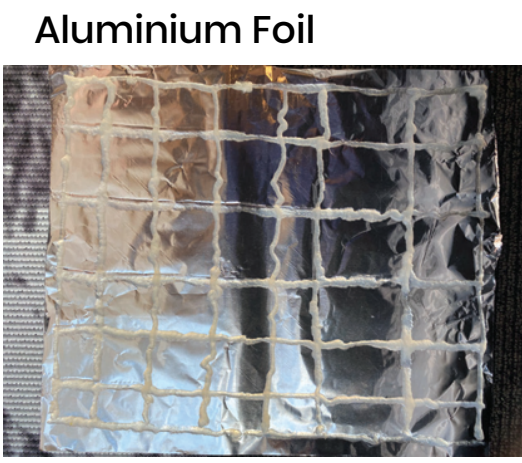

After 7 Days

Georgette - Pattern 1

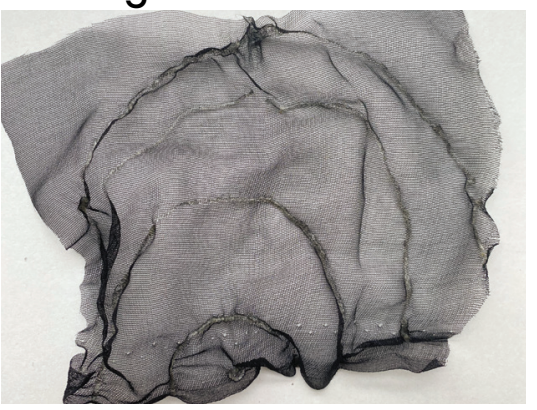

After 7 Days

Cotton - Pattern 1

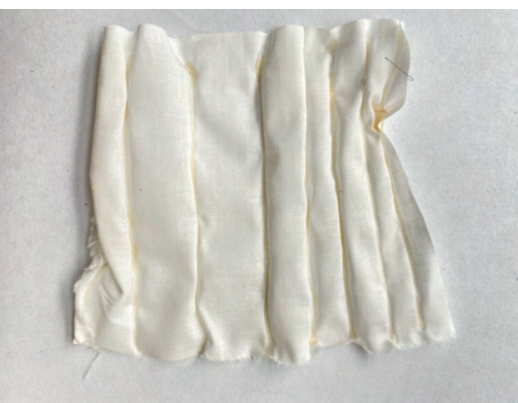

After 7 Days

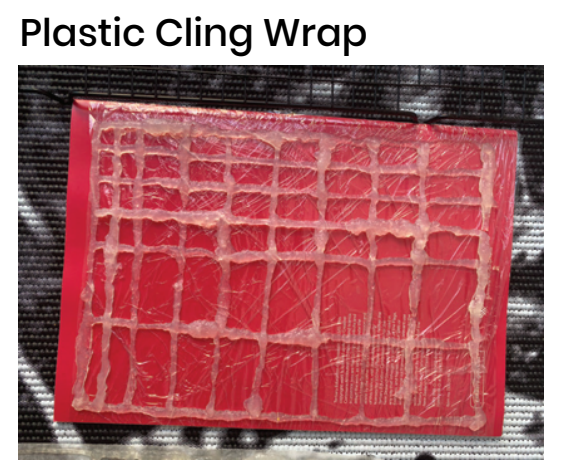

After 7 Days

\section{Georgette - Pattern 2}

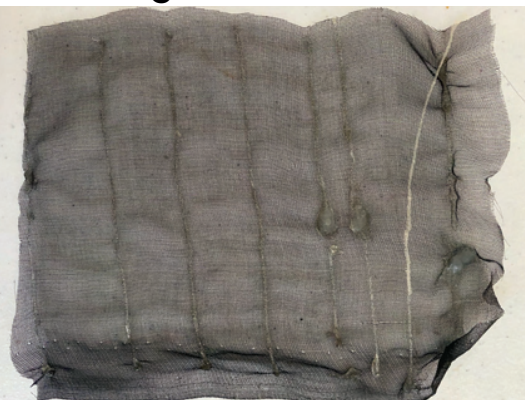

After 7 Days

\section{Cotton - Pattern 2}

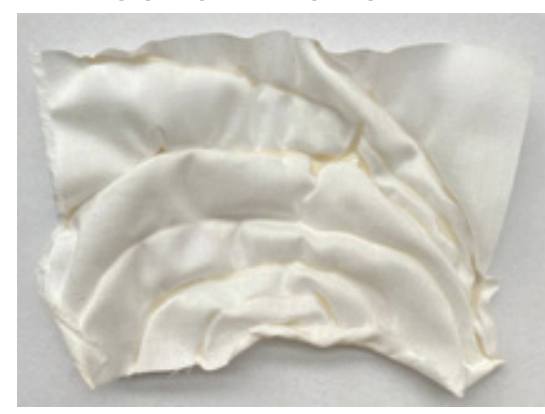

After 7 Days
Muslin

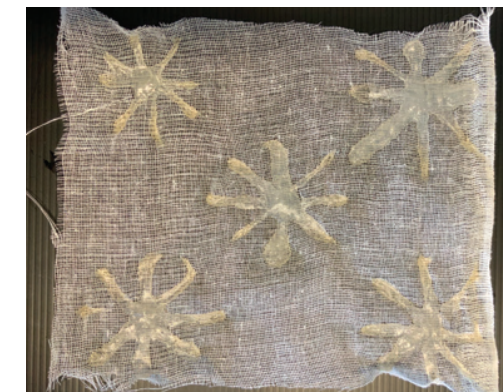

Upon Extrusion

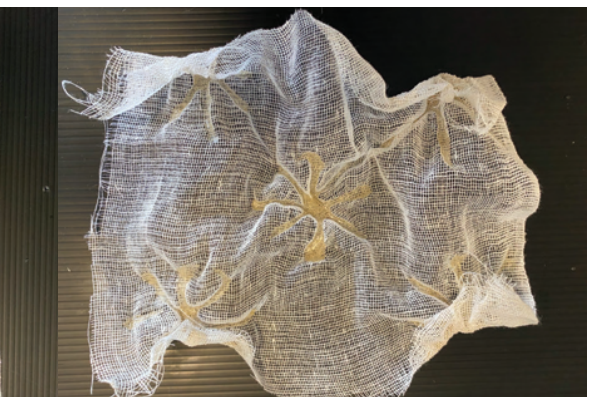

After 7 Days

Note: Author's work (2021)
The two design parameters investigated were;

- The ability to extract the dried semi-liquid c ulture extrusion from the surface without damage

- Surface shrinkage and material shrinkage once the extruded semi-liquid culture has dried 
The observation summary is tabulated in Table 3.1.

TABLE 3.1

Observations with respect to the two design opportunity parameters

\begin{tabular}{|c|c|c|c|}
\hline \multirow[t]{2}{*}{ Surface Material } & \multirow{2}{*}{$\begin{array}{l}\text { Ability to remove } \\
\text { from the surface }\end{array}$} & \multicolumn{2}{|c|}{ Visible shrinkage upon dry } \\
\hline & & $\begin{array}{c}\text { Dried semi-liquid } \\
\text { culture }\end{array}$ & Surface \\
\hline Baking Paper & Very Good & Yes & No shrinkage \\
\hline Tissue Paper & Poor & No & Low shrinkage \\
\hline Aluminium Foil & Poor & No & Very low shrinkage \\
\hline Plastic Cling Wrap & Reasonably Good & Yes & No shrinkage \\
\hline Georgette & Poor & No & Medium shrinkage \\
\hline Cotton & Poor & No & Medium shrinkage \\
\hline Muslin & Poor & No & High shrinkage \\
\hline
\end{tabular}

Note: Author's work (2021)

Based on these observations, it could be determined that some surfaces bond with the semi-liquid culture and shrink, forming different shapes. The other types of surfaces were unable to hold the semi-liquid culture paste when dried and so the extruded material itself shrinks and could easily be extracted from the surface.

These are two different design opportunities for further research. Of the two, I decided to conduct further experiments on the shrinkage characteristics of the substrates that can be observed once the semi-liquid culture is extruded onto a surface, as it has more potential for a collection of fashion garments.

The next stage of experiments studied the behaviour of fabric surfaces similar to muslin, as it showed excellent shrinkage characteristics while being more sustainable because it is made from natural fibres. 


\section{STAGE 4 - BEHAVIOUR ON FABRIC SURFACES MADE FROM NATURAL FIBRES}

The next stage consisted of experiments based on cotton rug-liner and muslin surfaces, once the mycelium-inoculated semi-liquid culture was extruded onto them using a plastic syringe.

Both these materials are made of natural fibres. The density of fibres in cotton rug-liner material is significantly lower than that of muslin. As for the previous stages where mycelium was involved, all the equipment and fabric surfaces were sterilised using a microwave oven before the mycelium inoculation. Once the mycelium was inoculated a semi-liquid culture was extruded onto the selected surfaces, the containers were immediately sealed using Micropore tape and kept inside a dark chamber for 14 days to complete the incubation of mycelium.

Combining the initial experience gained in the first experimental phase on mycelium growth in fabric mediums soaked in PDA, these experiments were conducted. Figure 3.19 demonstrates the observations. As an additional comparison opportunity, one surface sample was made by combining muslin and tissue paper, side-by-side.

\section{FIGURE 3.19}

Mycelium Growth on Fabric Surfaces Using the Extruded Semi-Liquid Culture

\section{Cotton rug-liner}

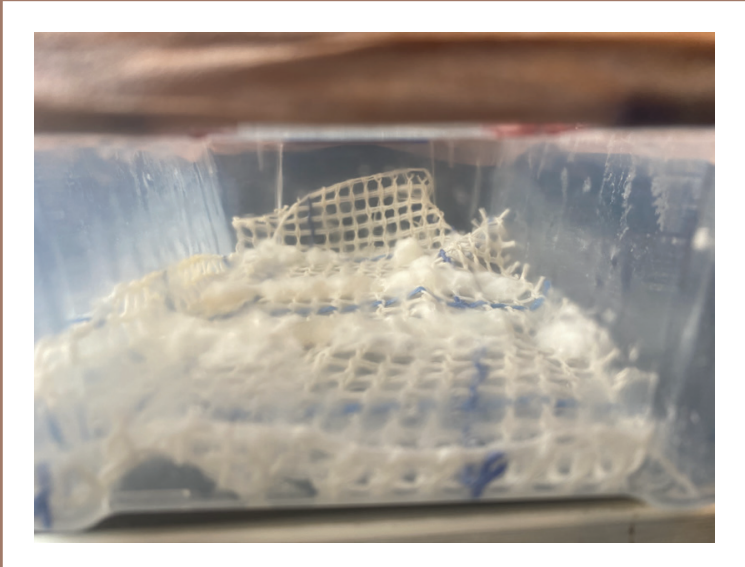

Upon Extrusion

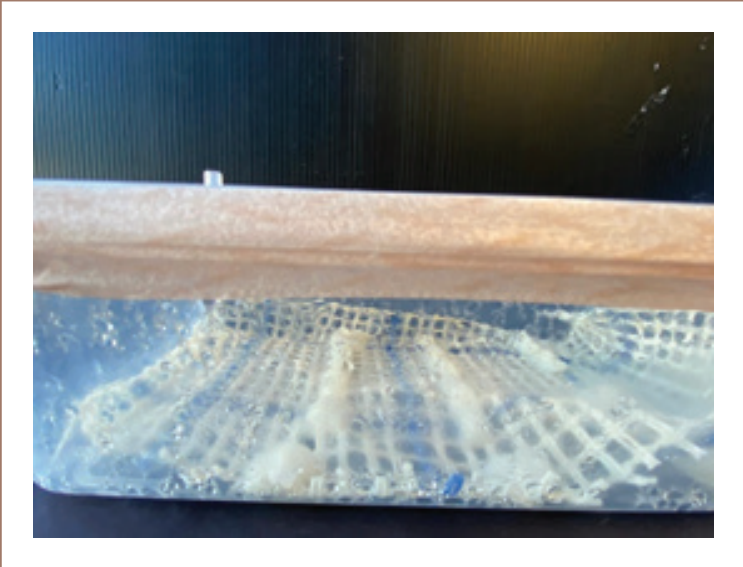

After 14 Days 


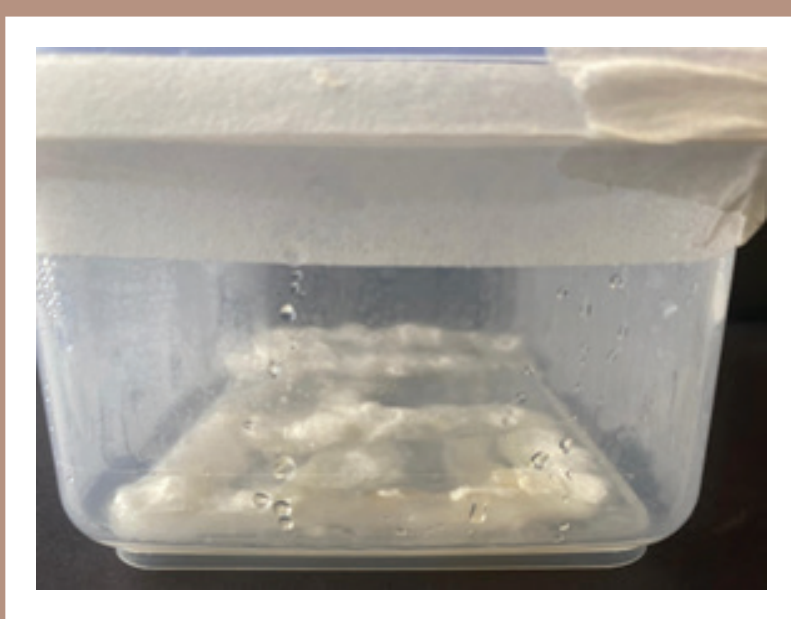

Upon Extrusion

\section{Muslin-Tissue Paper (side-by-side)}

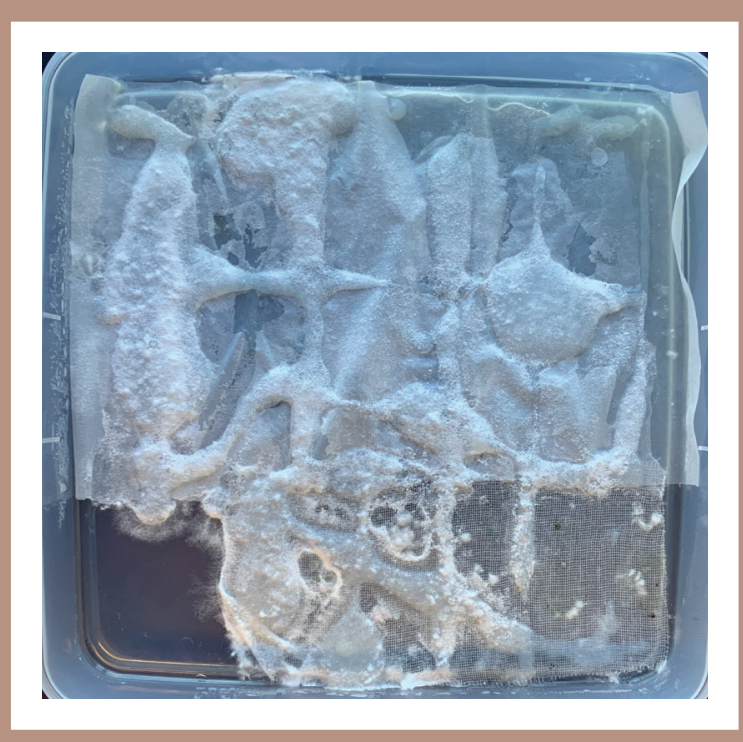

After 14 Days Note: Author's work (2021)

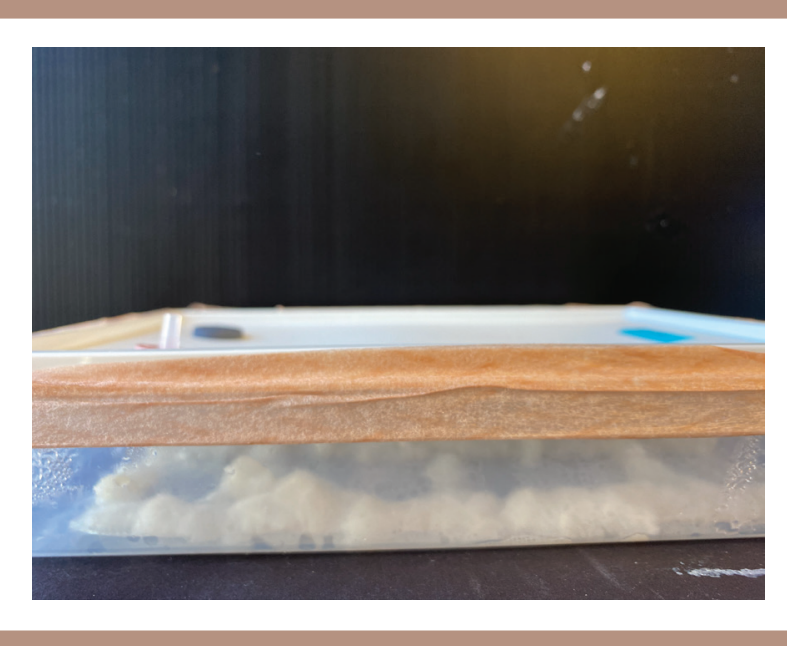

After 14 Days

With this set of experiments, the second step in the syringe printing experiments developed based on MMD, the fabrication (turning the growth form into desired shapes, products, or designs) was concluded.

Successful mycelium growth and intended design implication characteristics could be achieved using the semi-liquid culture developed and the optimum fabric surface types for manual syringe printing.

To complete the third step of desiccation, the baking process of the finished product as per the MDD method, the fifth experimental stage was initiated to bake the outcomes from the fourth experimental stage of the second phase. This is discussed in the next section. 
FIGURE 3.20

The laboratory drying oven used for desiccation

\section{STAGE 5 - THE DESSICATION}

Mycelium incubated three material samples obtained from the previous stage, the cotton rug-liner piece, the muslin piece and the combined surface of muslin and tissue paper, were placed inside an oven.

The temperature was set to $80 \mathrm{OC}$ and all the samples were baked for 120 minutes using a laboratory drying oven (shown in Figure 3.20) to desiccate and kill the organisms.

Obtained samples after the baking process are shown in Figure 3.21 and Figure 3.22.

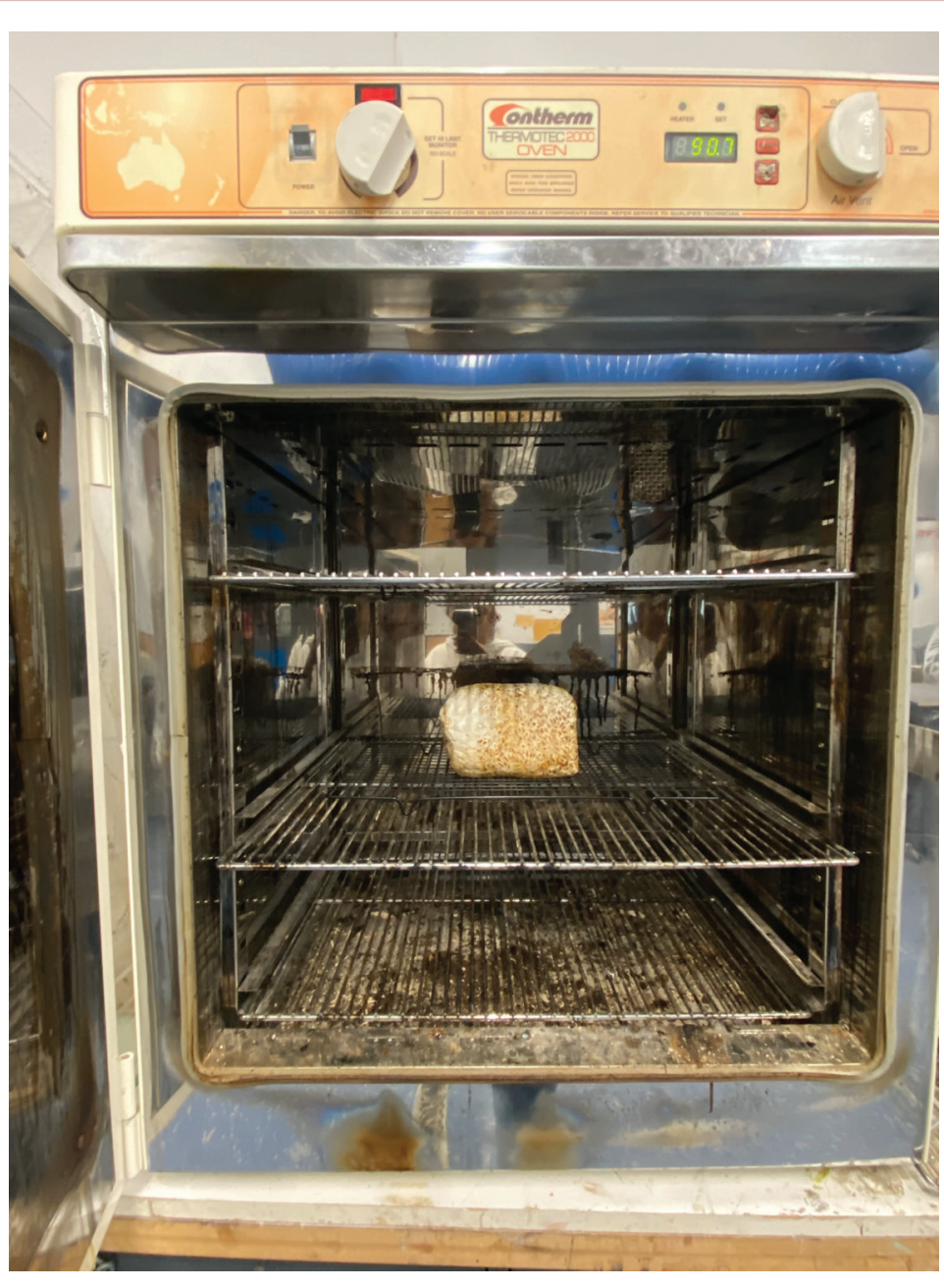

Note: Photograph taken by the author (2021) 
Baked products using grown mycelium on fabric surfaces soaked in PDA

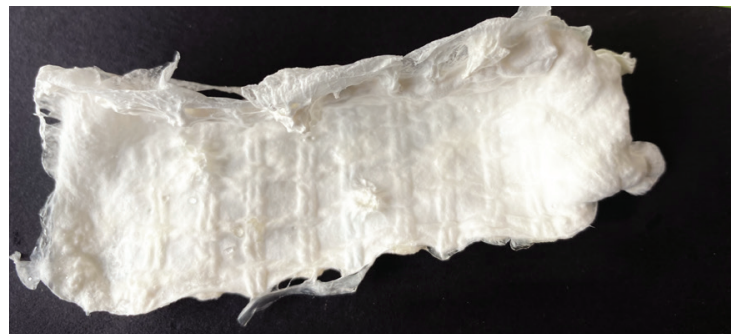

Turkey Tail mycelium on PDA soaked cotton rug

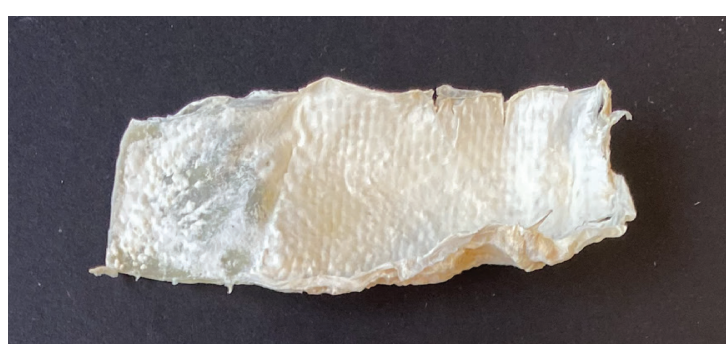

Grery Oyster mycelium on PDA soaked Jute

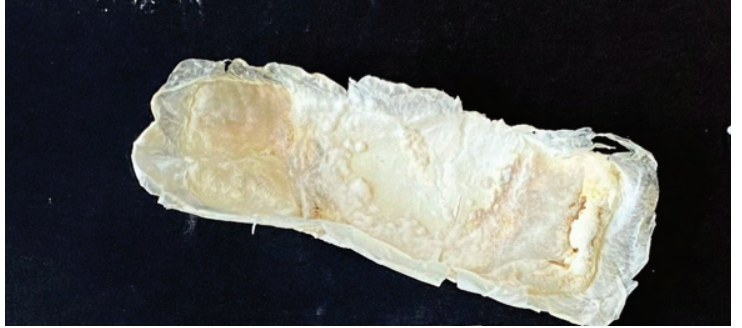

Turkey Tail mycelium on PDA soaked dyed Jute

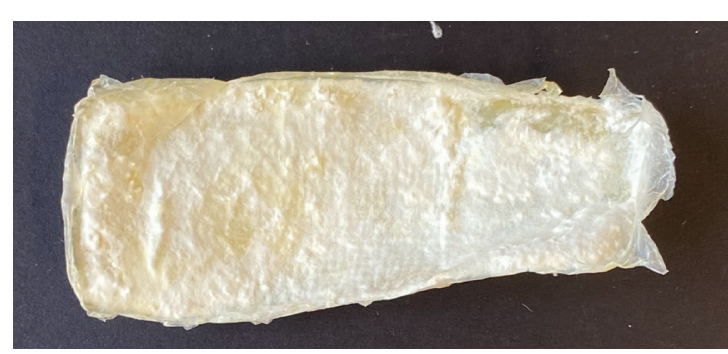

Grey Oyster mycelium on PDA soaked dyed Jute

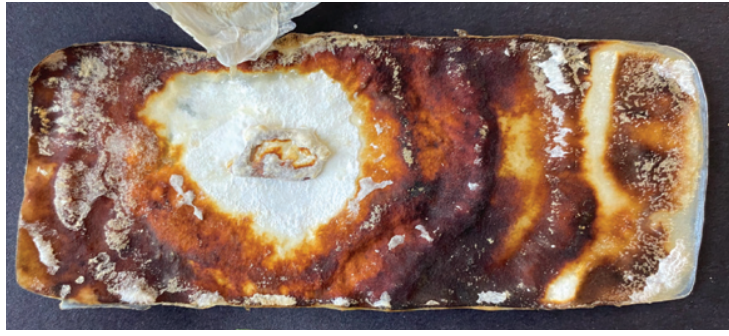

Turkey Tail mycelium on PDA soaked dyed Jute

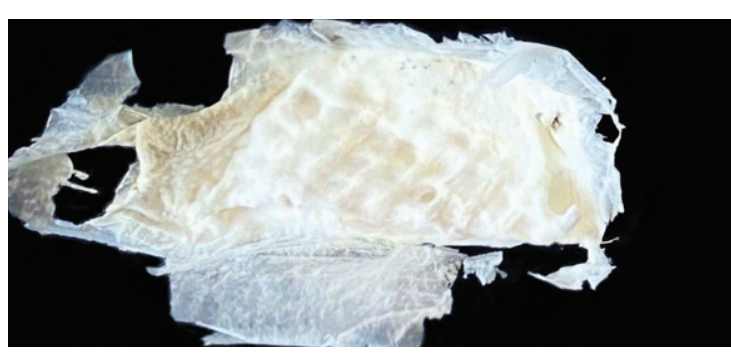

Grey Oyster mycelium on PDA soaked cotton rug

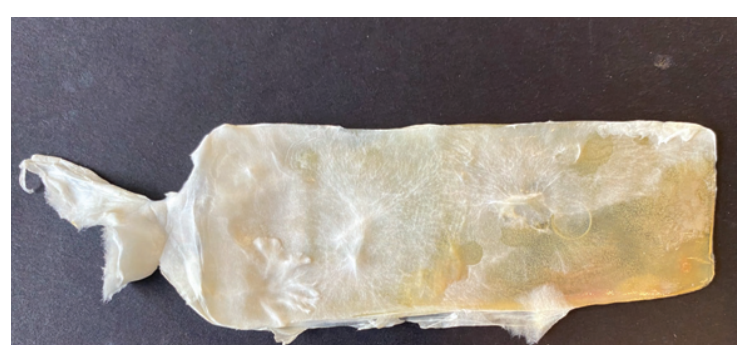

ICPM 15546 mycelium on PDA soaked cotton

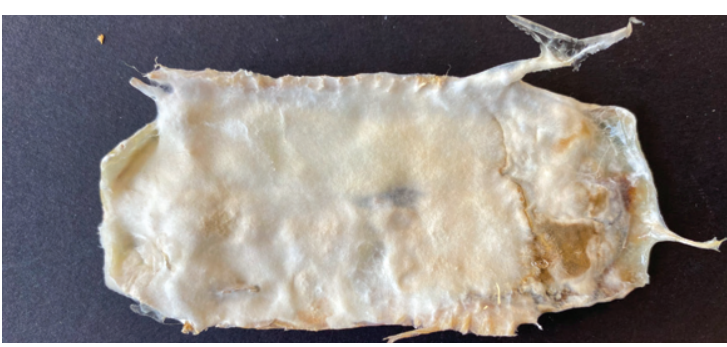

Ganoderma mycelium on PDA soaked dyed Jute

Note: Author's work (2021) 
FIGURE 3.22

Baked products using grown mycelium through the extruded semi-liquid culture on fabric surfaces

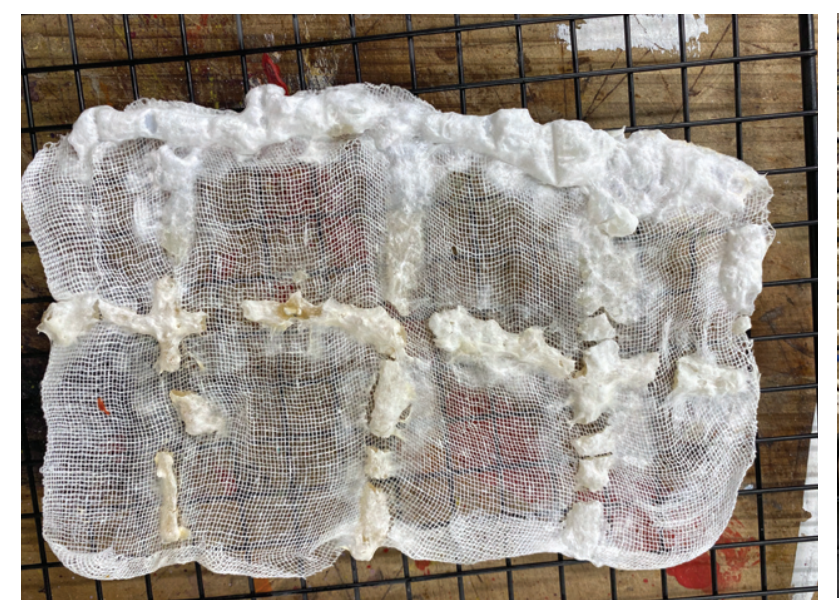

On muslin surface

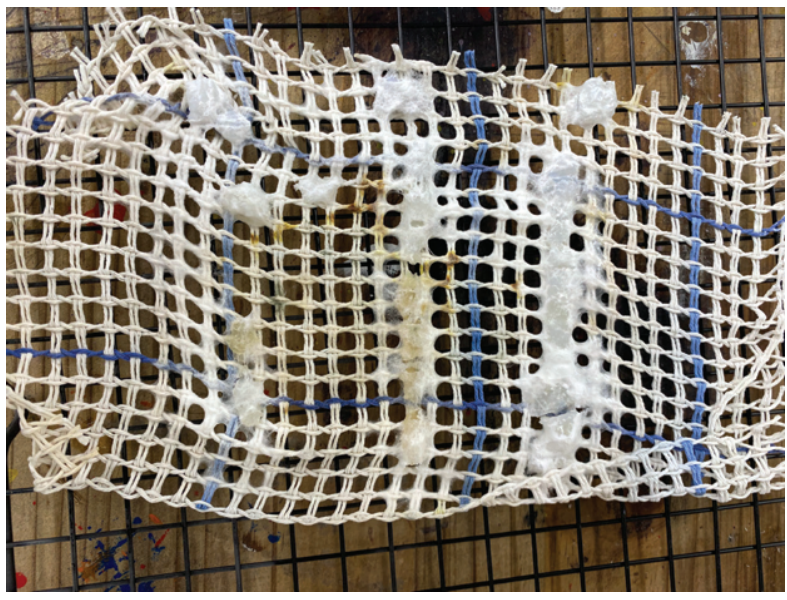

On cotton rug-liner surface

Note: Author's work (2021)

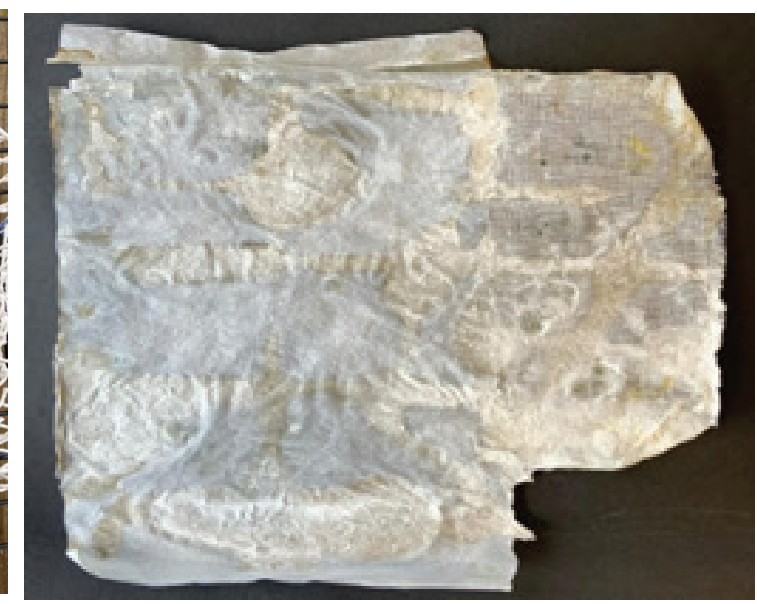

On muslin - paper combined surface

With the successful observations and results obtained from the syringe printing techniques, the experimental research was then moved forward to its next progressive stage, the use of digitally designed, 3-dimensional printing techniques.

Here, the robotic arm extruder was used to create patterns with the developed semi-liquid culture. This will be discussed in detail from the next chapter onwards. 
DIGITAL/COMPUTER NUMERIC CONTROL (CNS) EXPERIMENTS

- INTRODUCTION - WORKFLOW STRUCTURES FOR DIGITAL/CNC EXPERIMENTS - PAPM - POTATO AGAR PRINTABLE MEDIA EXPERIMENTS - MIPM - MYCELIUM INOCULATED PRINTABLE MEDIA - EXPERIMENTS ON SHRINKAGE ANALYSIS $\diamond$ EXPERIMENTS ON SHRINK

KEY WORDS

PAPM - Potato Agar Printable Medic

MIPM - Mycelium Inoculated Printable Media

CHAPTER 


\section{DIGITAL/COMPUTER NUMERIC CONTROL (CNC) EXPERIMENTS}

\section{INTRODUCTION}

Moving to the next experimental phase, a robotic arm printer available in the 3D modeling workshop of Victoria University of Wellington was used with an extruder designed to perform extrusion of fluid materials.

Similar to the precedence experimental studies that used the syringe printing on a robotic arm, trials were conducted involving extrusion of bio-composite material onto fabric substrates using the device ABB IRB 6700, shown in Figure 4.1.

A series of experiments were conducted that are sub-categorised as extrusions with printable agar medium and extrusions with mycelium inoculated agar.
FIGURE 4.1

ABB's IRB 6700 robotic arm printer

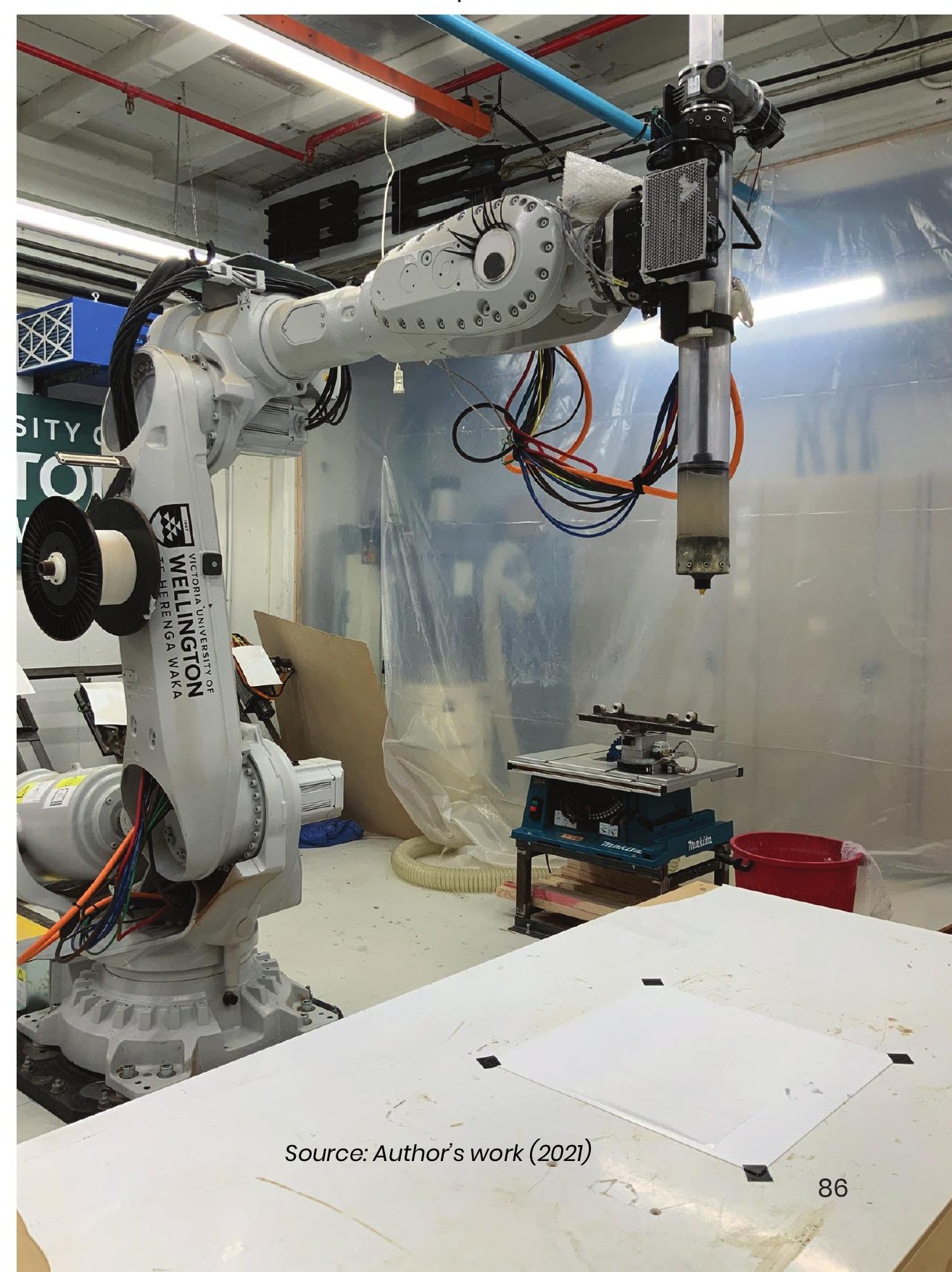


The robotic arm printer possesses the following special technical characteristics, which makes it a handy tool in the generation of 3D printed objects.

- Spare heads for interchanging locally manufactured various 3D printing assemblies, enabling printing experimentation with clay, Poly Lactic Acid, concrete, and other materials

- The slow-jogger mode for safe close interaction with operators, or in auto mode behind the safety fencing

\section{THE 4000ML EXTRUDER}

The $4000 \mathrm{ml}$ extruder (Figure 4.2) is driven by a large, closed-loop hybrid stepper motor with a 0.625 " Acme screw. This is a much more robust drive system. It's also capable of extruding thicker clay or paste.

With a larger tube and the larger nozzle outlet, it is capable of larger-diameter nozzles up to 0.5 " $(12.75 \mathrm{~mm})$. This gives us the ability to extrude higher volumes of clay through large nozzles using $3 d$ printing machines.

\section{FIGURE 4.2}

The $4000 \mathrm{ml}$ extruder used in the robotic arm printer

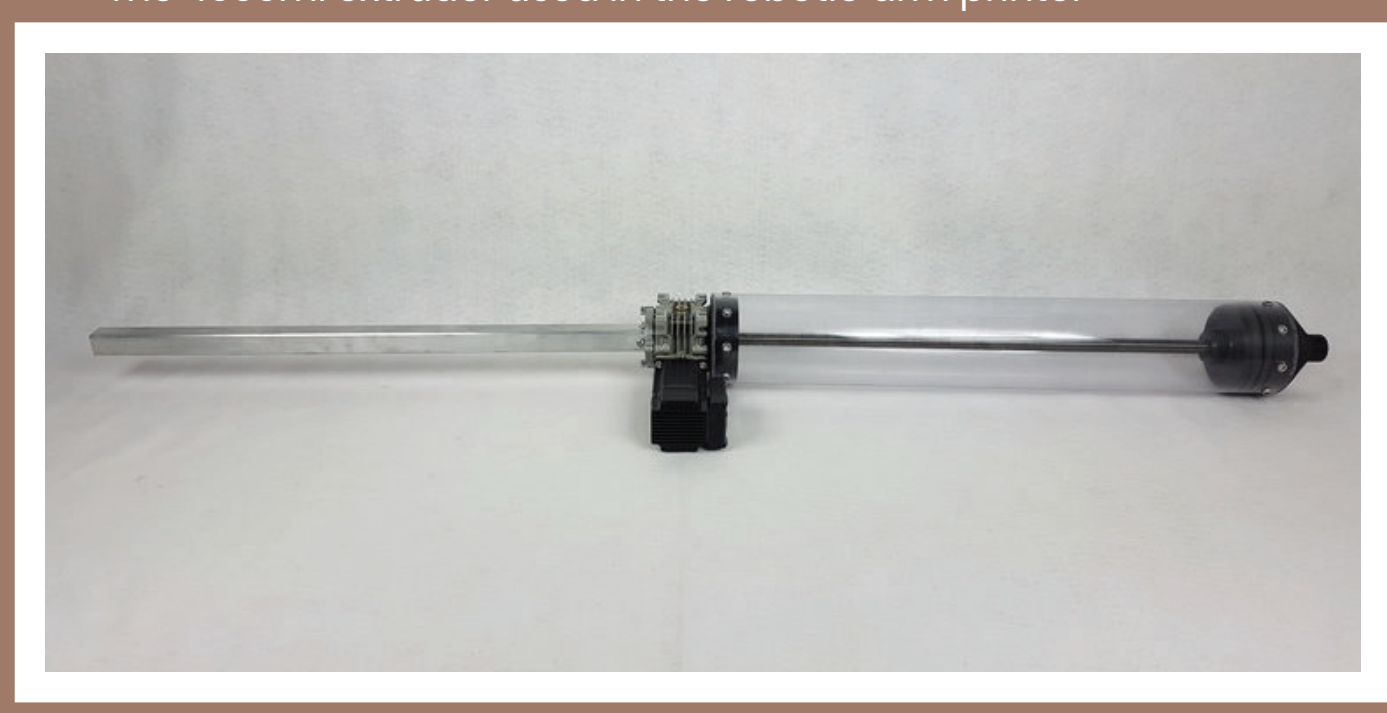

Source: https://3dpotter.com/extruders/4000-extruder 


\section{LOADING OF THE ROBOTIC ARM EXTRUDER WITH PRINTABLE AGAR MEDIUM}

The step-by-step loading procedure of printable agar medium into the extruder and fixing it in the robotic arm printer is illustrated in Figures 4.3 to 4.7.

FIGURE 4.3

Filling the Extruder with Printable Agar Medium

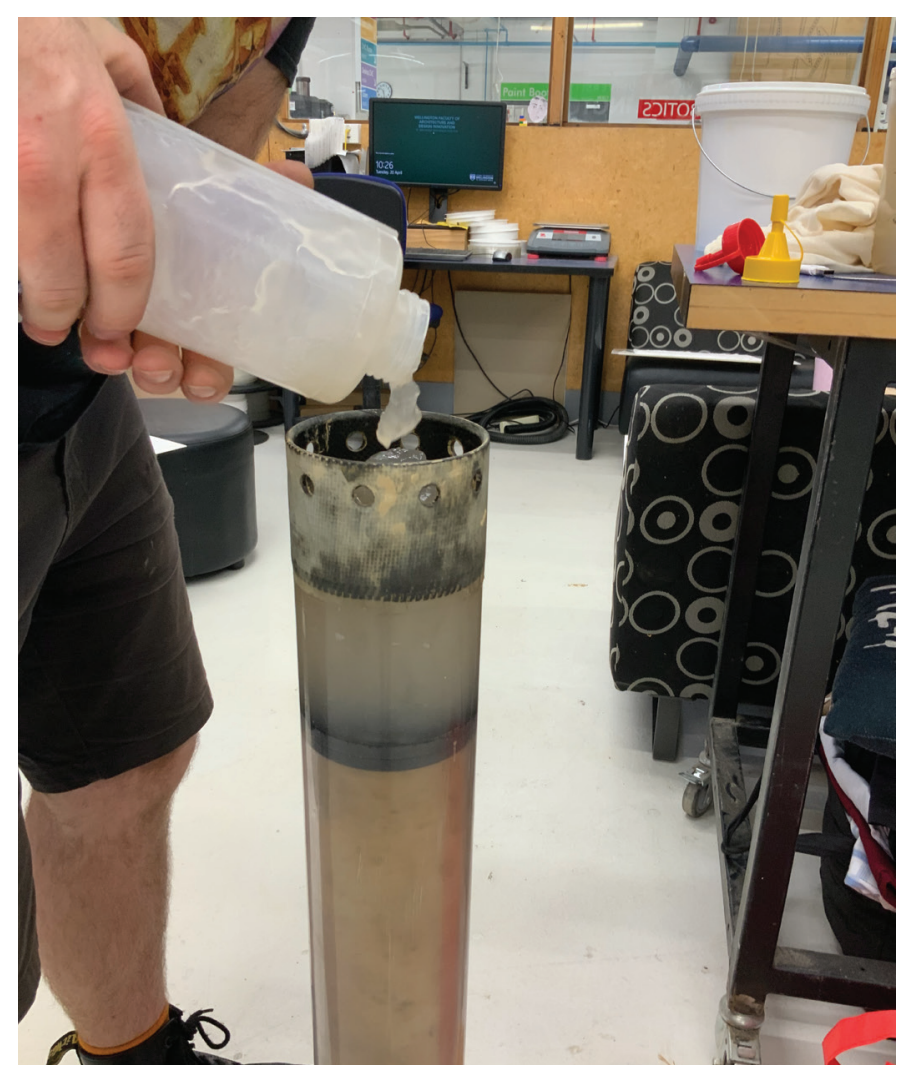

Source: Author's work (2021)
FIGURE 4.4

Fixing the $3 \mathrm{~mm}$ Diameter Nozzle Head to the Extruder
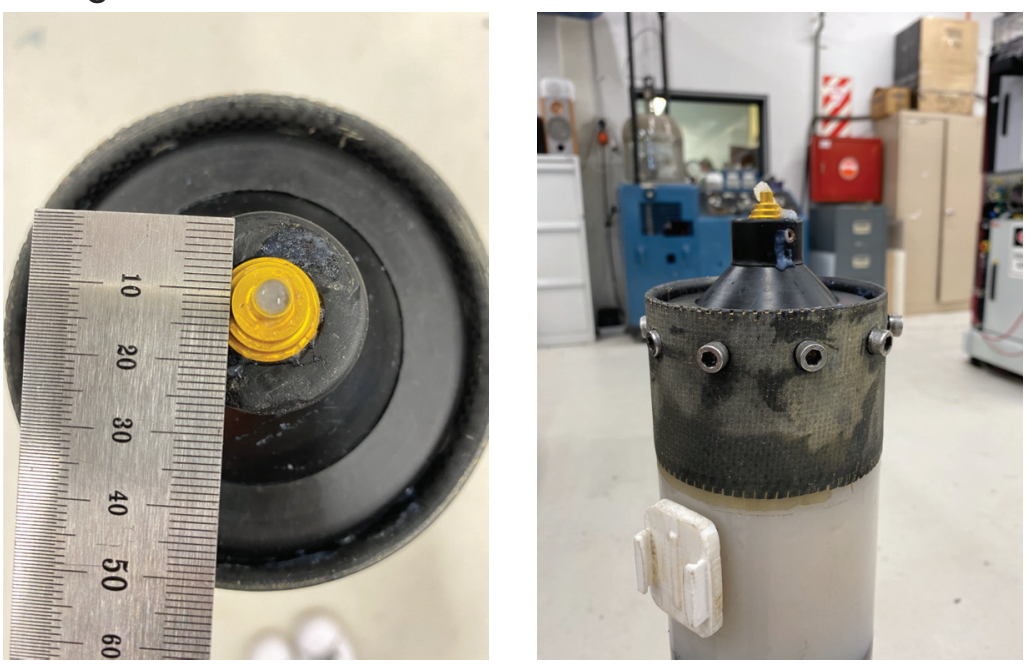

FIGURE 4.5

Fixing the Extruder to Axis 6 of the Robotic Arm Printer and Tightening it Using the Velcro Straps
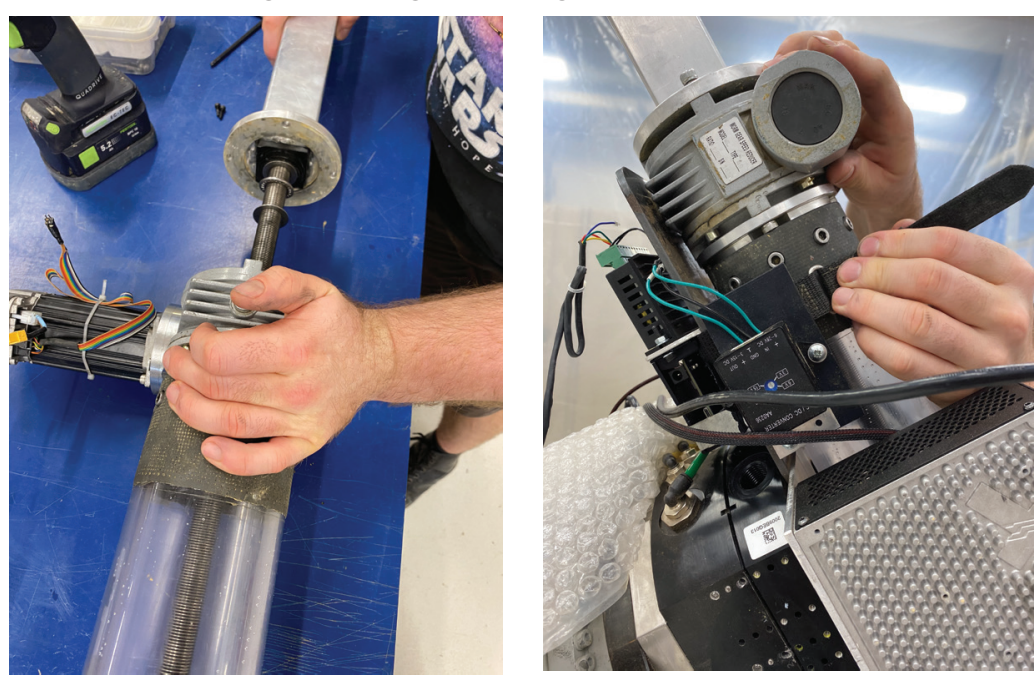

Source: Author's work (2021) 
FIGURE 4.6

Fixing the Controller Circuit Module into the Robotic Arm Printer

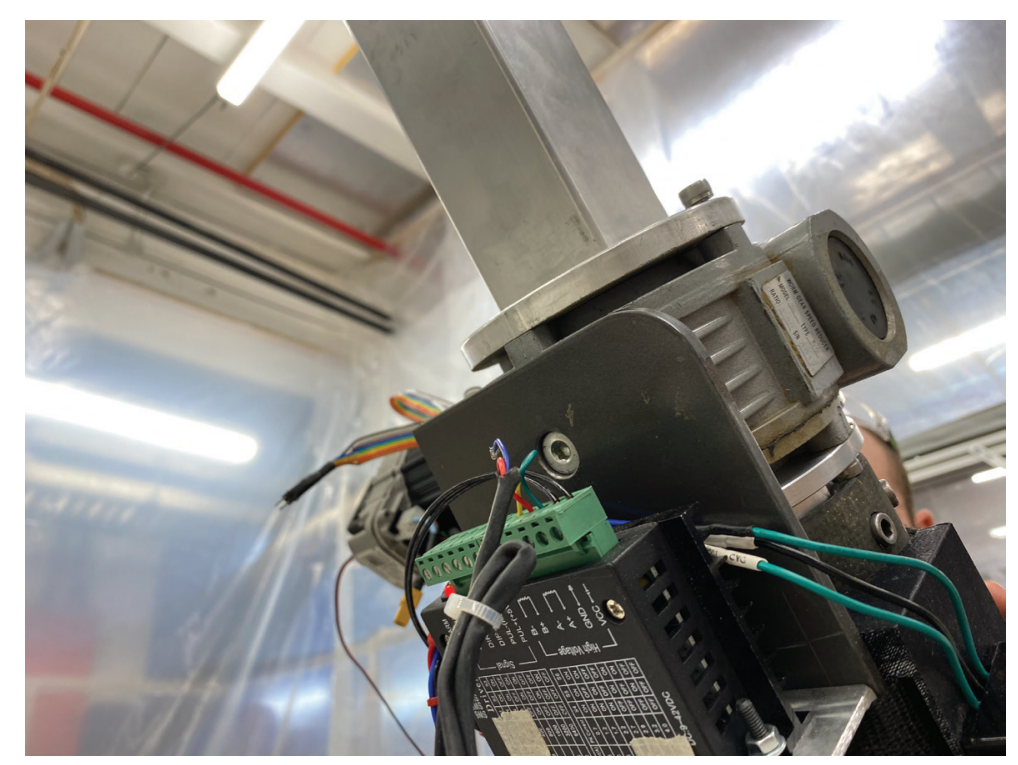

Source: Author's work (2021)
FIGURE 4.7

The Ready-To-Use Extruder Fixed Robotic Arm Printer with the FlexPendant interaction Panel

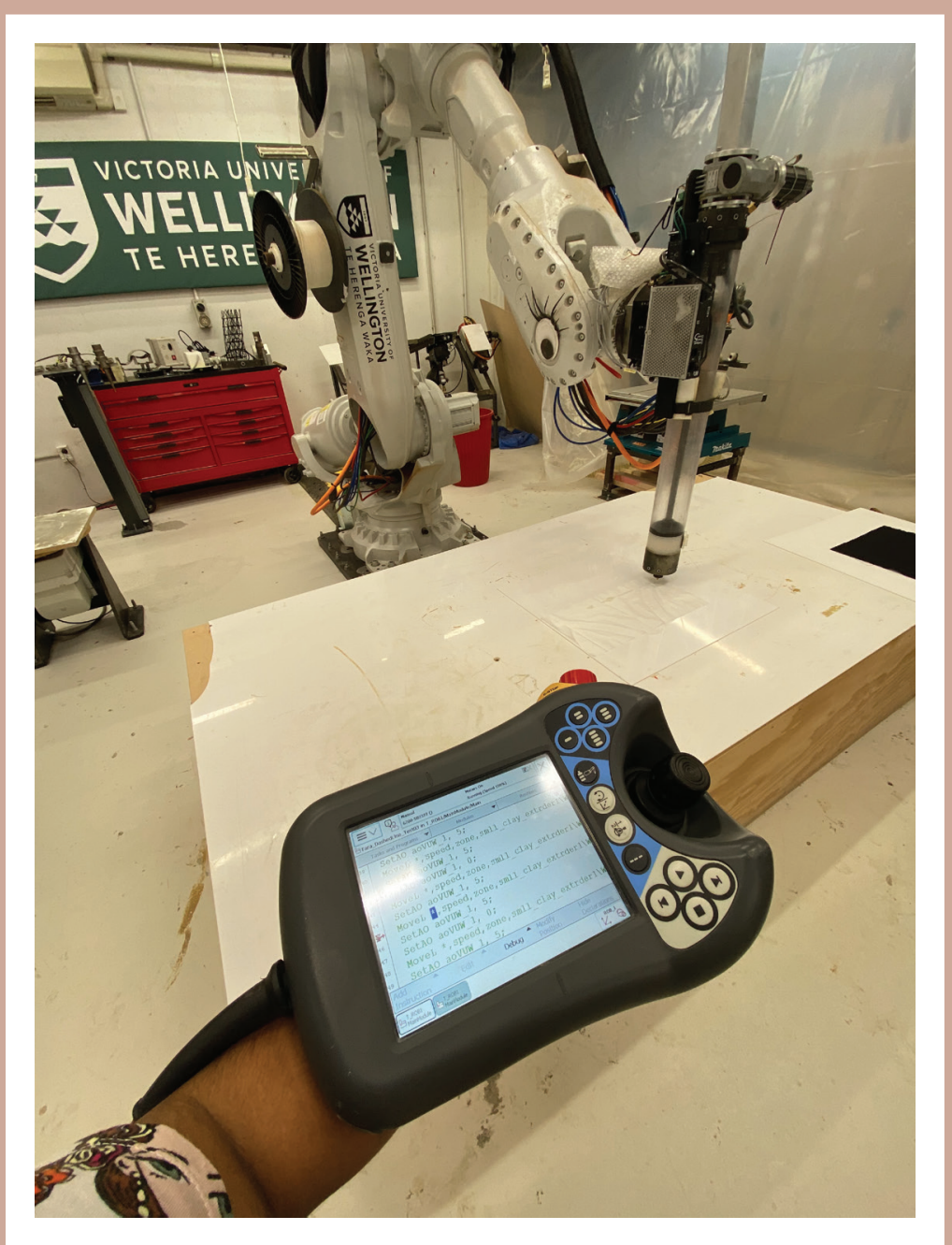

Source: Author's work (2021) 


\section{CONTROL AND OPERATION OF THE ROBOTIC ARM PRINTER}

The ABB robotic arm printer is controlled through a fifth-generation multi-robot controller with PC tool support called the ABB IRC5; this combines motion control, flexibility, modularity, usability, application interfaces, and safety while optimising the robot's performance for short cycle times and precise movements (ABB IRC5 Controller, n.d.).

The ABB IRC5 uses RobotWare, a family of configurable controller software designed to make operating a robot more productive and cost-effective. The core of RobotWare was developed on RAPID, a highly flexible programming tool and one of the most powerful software languages in the robot industry (ABB, 2004).

RAPID can be fed with user-customised plug-ins to perform different tasks such as accurate path-holding spot welding, material handling, and machine tending. It is the RAPID programming language that commands the tool-path for the robotic arm printer to direct and control its operational movement. 


\section{GRASSHOPPER}

Grasshopper is a graphical algorithm editor that is tightly integrated with the 3D modeling software Rhinoceros. Operating Grasshopper does not require much knowledge of programming or scripting, but still allows designers who are exploring new shapes using generative algorithms to build form generators from simple to complex designs (About Grasshopper, n.d.) through real-time parametric modeling.

Scripts, which are lists of replicable actions to be completed by a certain computing environment, are used in Grasshopper in generating designer shapes and forms. Scripting is basically about writing instructions for a certain application. Those instructions must come with a language, a syntax, and a vocabulary.

Learning scripting makes it very easy for designers to reduce the size and complexity of their definitions and develop solutions faster with less work, through the use of complex program-flow concepts (Molinos, 2014).

\section{DEFINING DIGITAL TOOL-PATHS FOR A ROBOTIC ARM PRINTER}

The objective of this experimental phase was to get the developed mycelium-based bio-composite mediums extruded onto paper and fabric surfaces through the digital three-dimensional printing technique.

As the first step, different extrusion patterns were designed and developed using the parametric design method through the PC software Grasshopper. Parametric design is a process where creative designs are developed based on the input of parameters, such as materials, site constraints, and even environmental issues. Design outcomes can be modified in real time by varying the design parameters, rather than applying modifications to the design object itself, which is the usual practice in the conventional method of generative design.

The script created through Grasshopper was then exported to the PC software ABB RobotStudio, which converted it to a RAPID programming code and sent it to the robotic arm extruder to start the printing process. 



\section{PIGMENTING THE PRINTABLE AGAR MEDIUM}

Since the development of the printable agar medium, it has been used in different experiments in its original colour, opaque. Through the broadening of design implication ideas, focus turned to how different colours could be added to the extruded patterns.

In opting for a sustainable approach, the introduction of colours was done using natural food powders. To perform some of the experiments in the following stages, a red colour printable agar medium was prepared by adding one teaspoon of beetroot powder into the mixture, see Figure 44. These natural powders are made by freeze-drying fresh beetroot and then milling them into powder while maintaining the vibrant colour.

The preparation of the red-coloured printable agar medium is shown in Figure 4.8.

\section{FIGURE 4.8}

Preparation of the Red-Coloured Printable Agar Medium

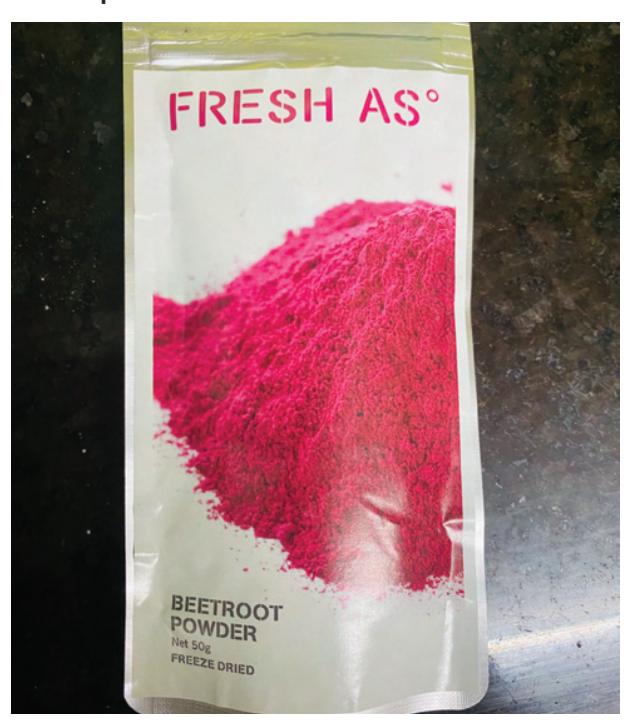

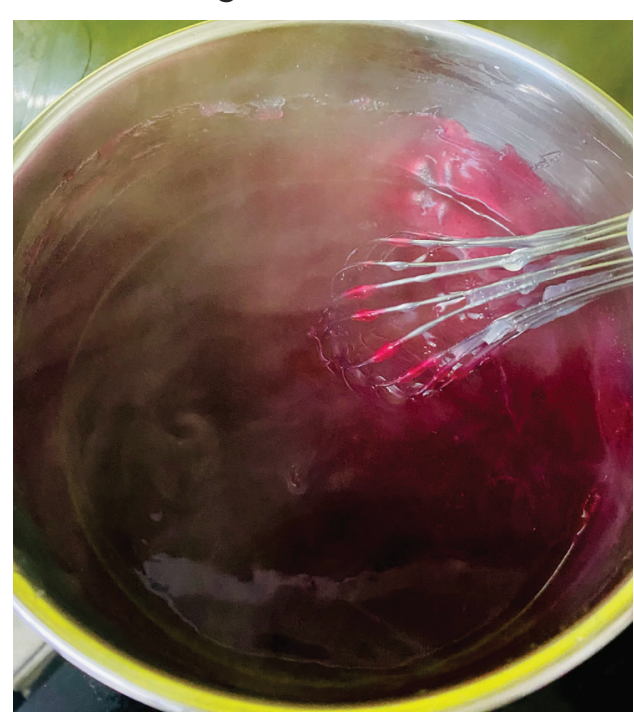

Source: Author's work (2021)

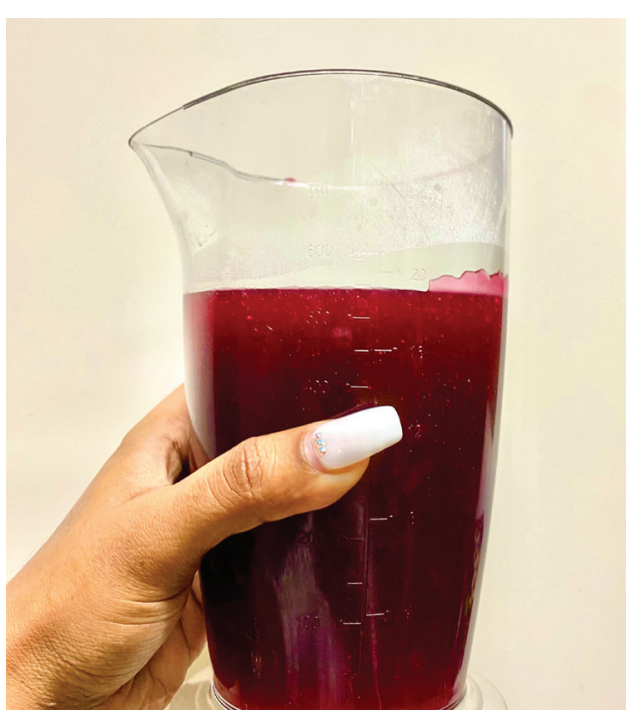




\section{PAPM - POTATO AGAR PRINTABLE MEDIA EXPERIMENTS}

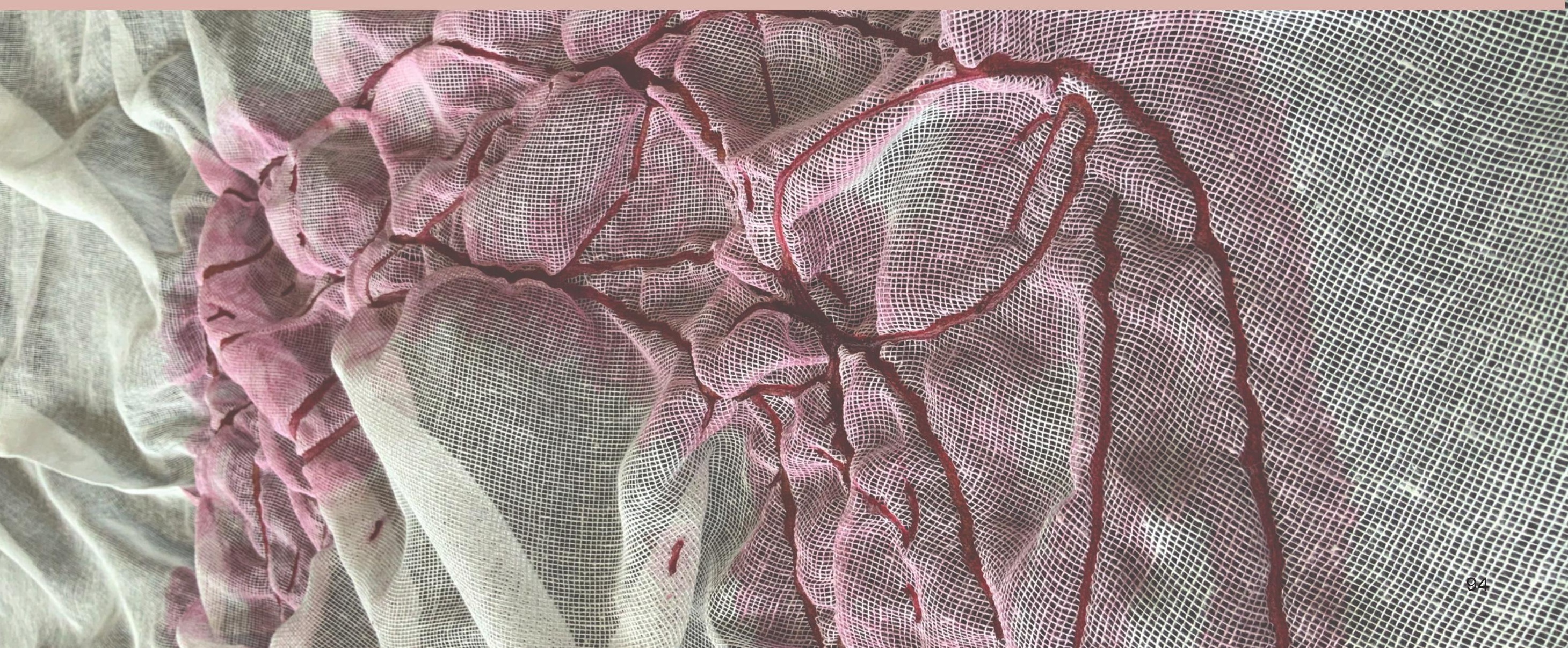


FIGURE 4.9

Workflow diagram of digital extrusion exercises conducted using the printable agar medium on paper and fabric surfaces

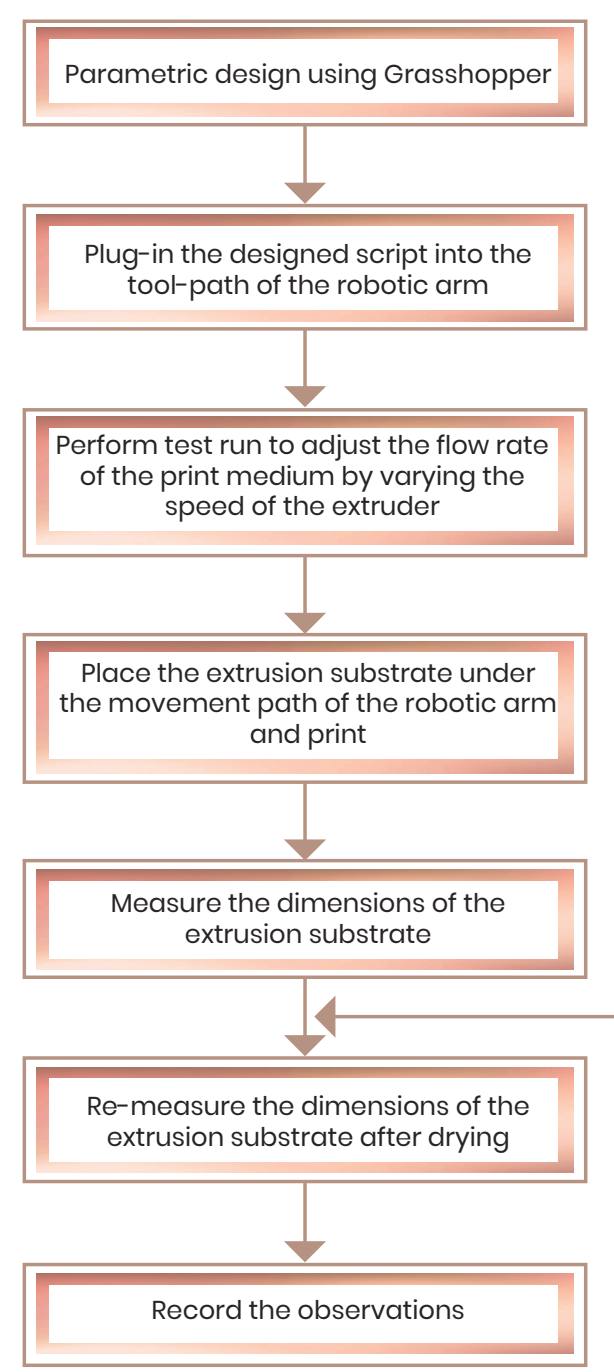

Source: Author's work (2021)

\section{APM - AGAR PRINTABLE MEDIA EXPERIMENTS}

Here, the developed printable agar medium was extruded onto different surfaces of paper and fabric. Figure 4.9 illustrates the typical workflow diagram of the extrusion exercises. Different extrusion patterns were generated through parametric design using the software Grasshopper. Results and observations are demonstrated in the following 


\section{TEST RUN AND SPEED ADJUSTMENTS}

As mentioned in the third step of the workflow diagram (Figure 4.9), the operating speed of the robotic arm was required to be adjusted to obtain the extrusion pattern for the design.

Depending on the viscosity of the printable agar medium the rate of extrusion could be varied. As the material flow through the extruder was constant, desired patterns could be obtained without smudging, by increasing the speed of operation of the robotic arm.

Figure 4.10 illustrates the outcomes of the test run that were conducted.

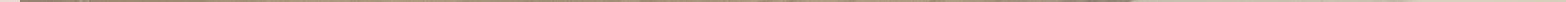




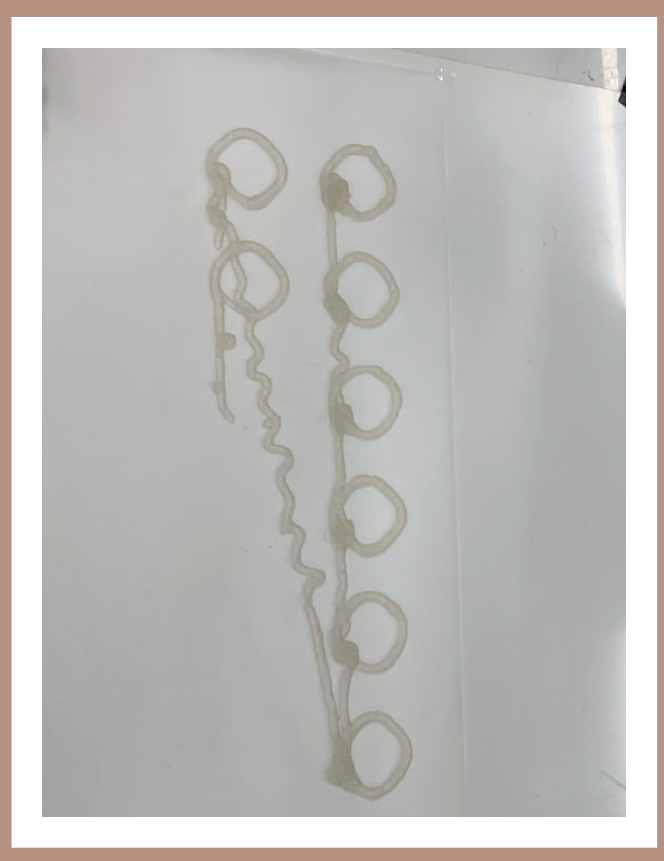

Slow movement of the extruder.

Too much printable agar medium on the substrate.
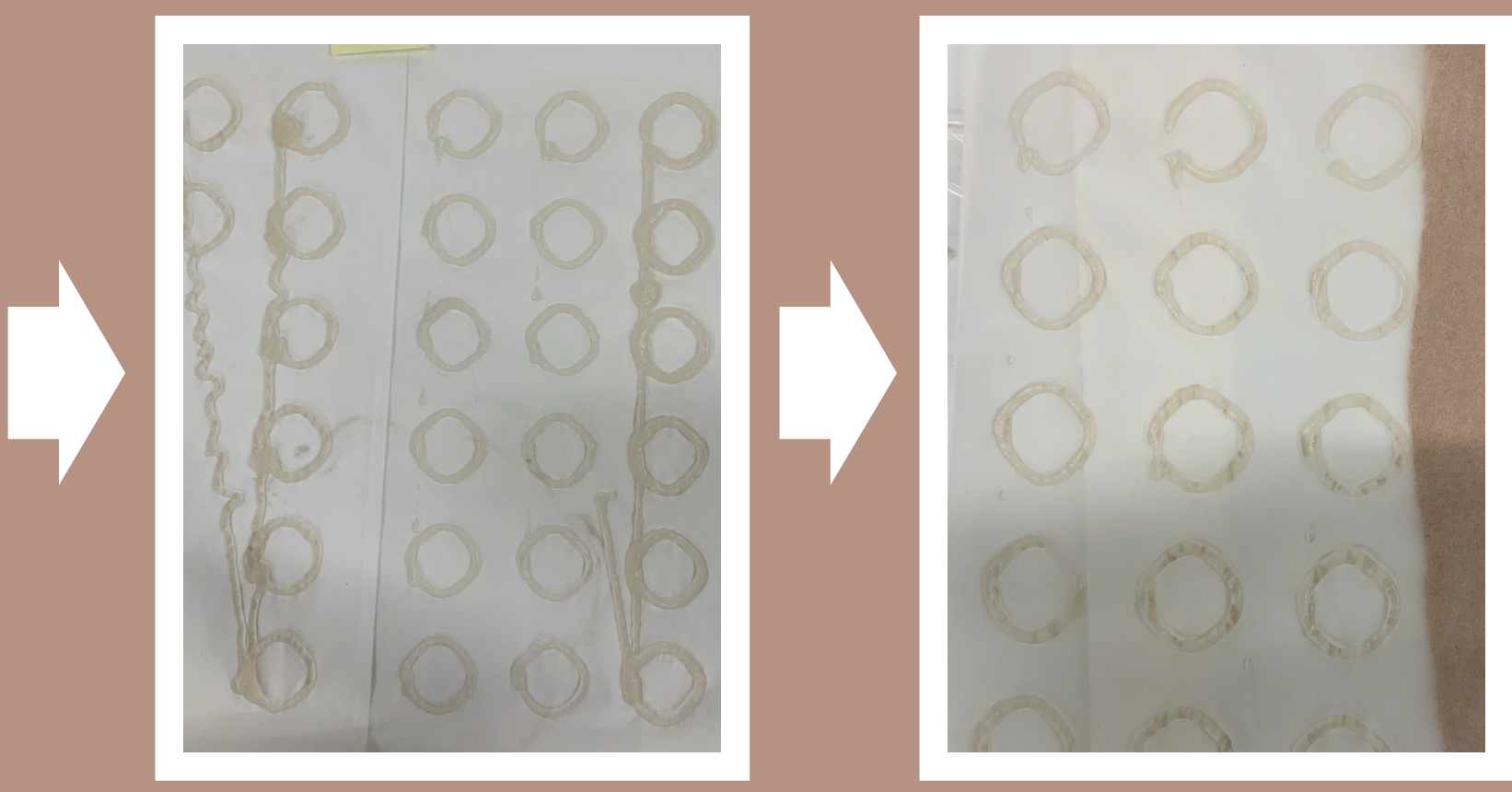

Speed incresead to avoid the spilling of print medium during the diagonal movement of the robotic

arm.

Source: Author's work (2021)
Speed incresead further to obtain the perfect extrusion pattern. No smudging of printable agar medium. 


\section{EXTRUSION PATTERN NUMBER 1}

Selected substrates:

Jute, Cheese Cloth, Muslin, Baking Paper, Tissue

FIGURE 4.11B

Developed Grasshopper Script

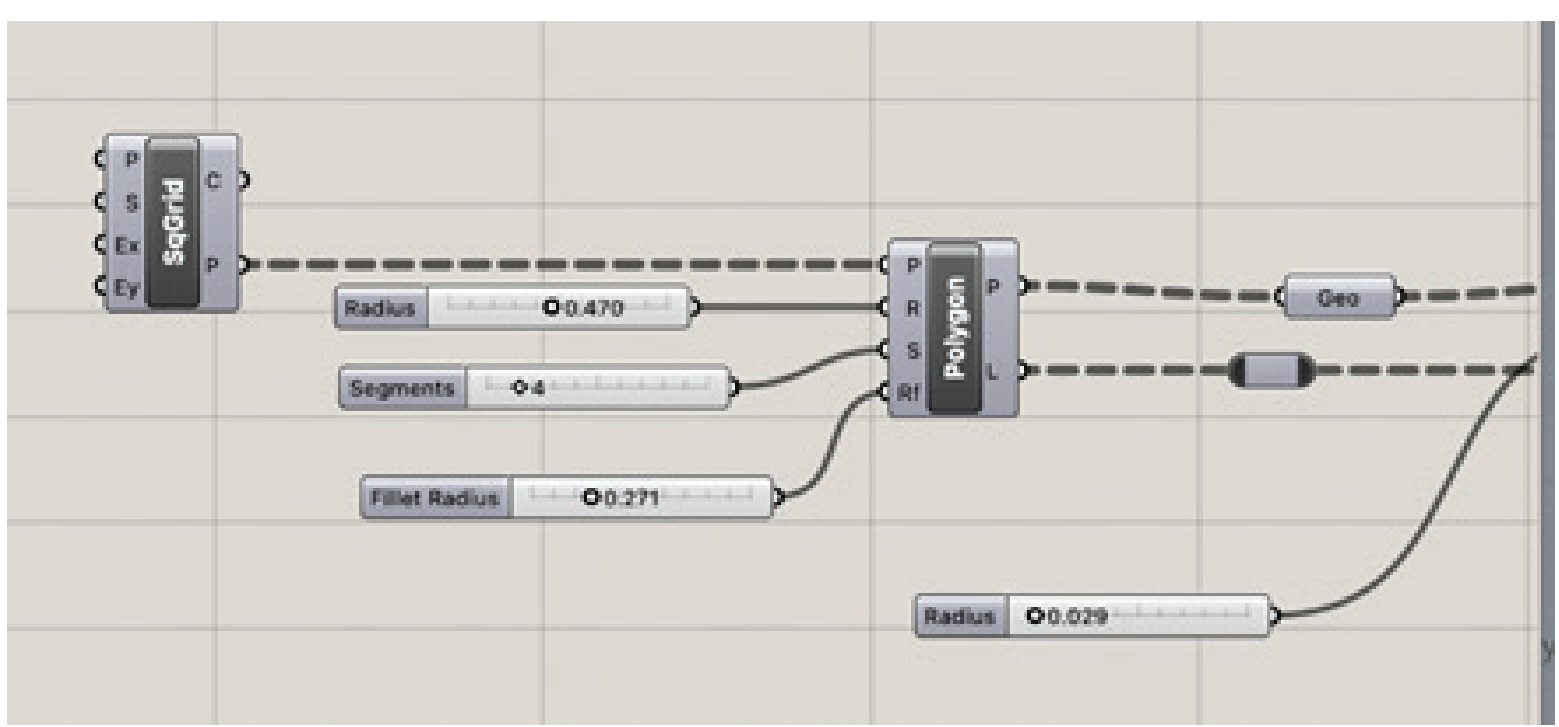

FIGURE 4.11A

\section{Extrusion Pattern}

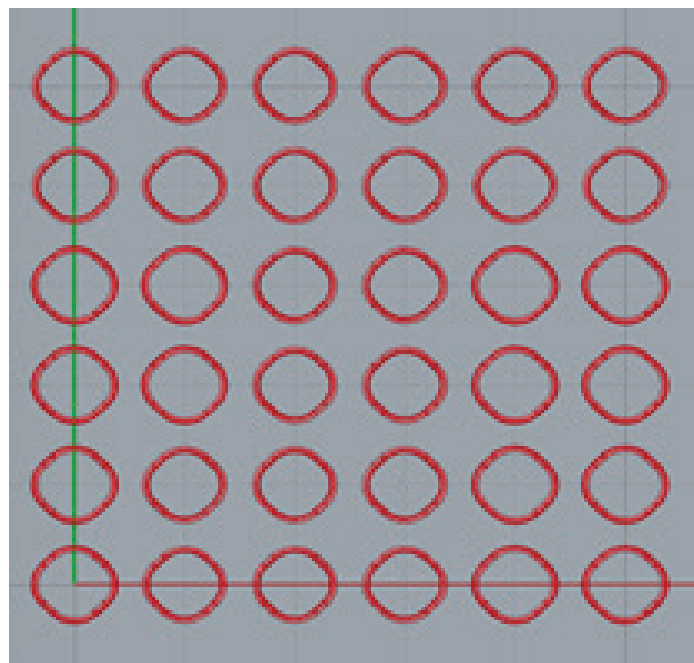

Source: Author's work (2021) 
FIGURE 4.11C

Observations of the First Pattern on Different Substrates Upon Extrusion

Substrate:
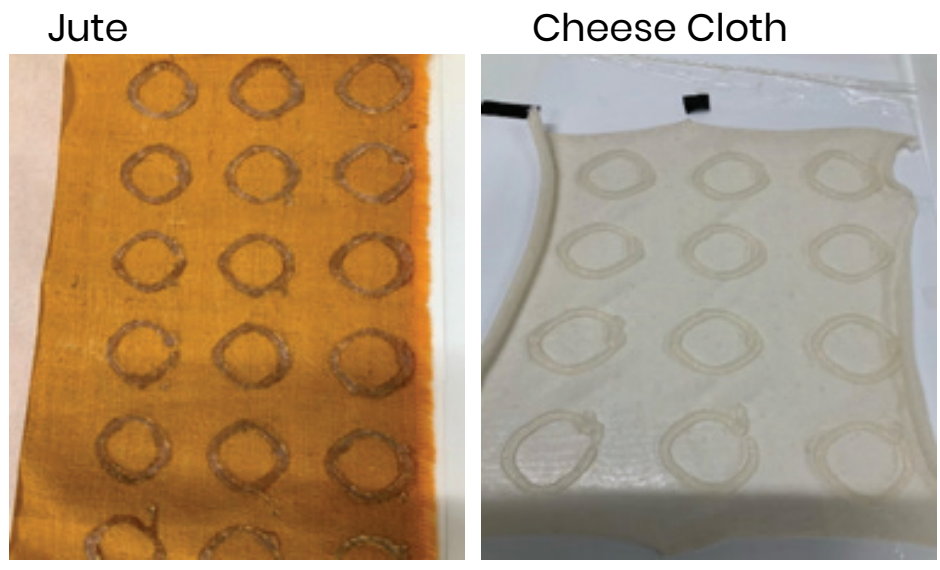

\section{Muslin}

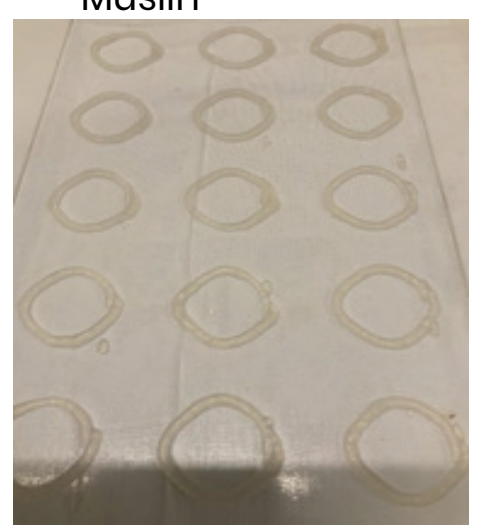

Before drying

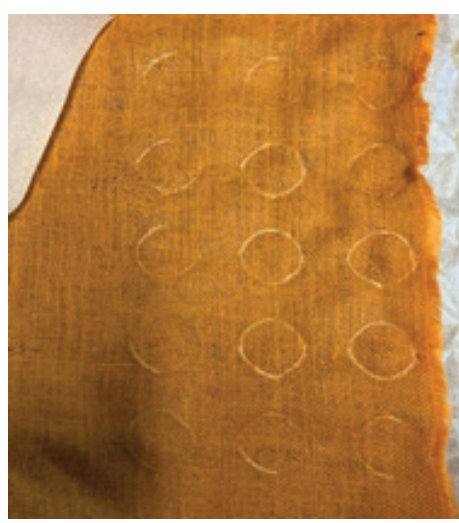

After drying
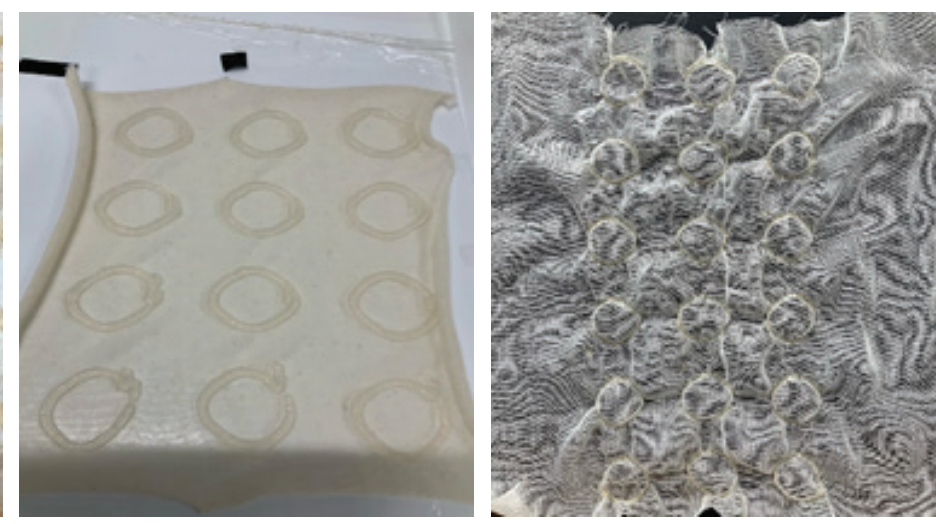

Source: Author's work (2021)

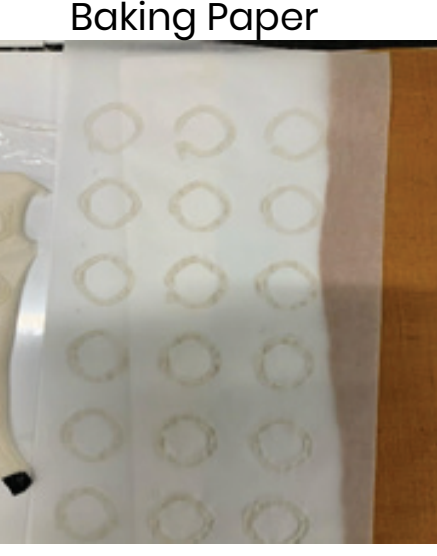

Tissue
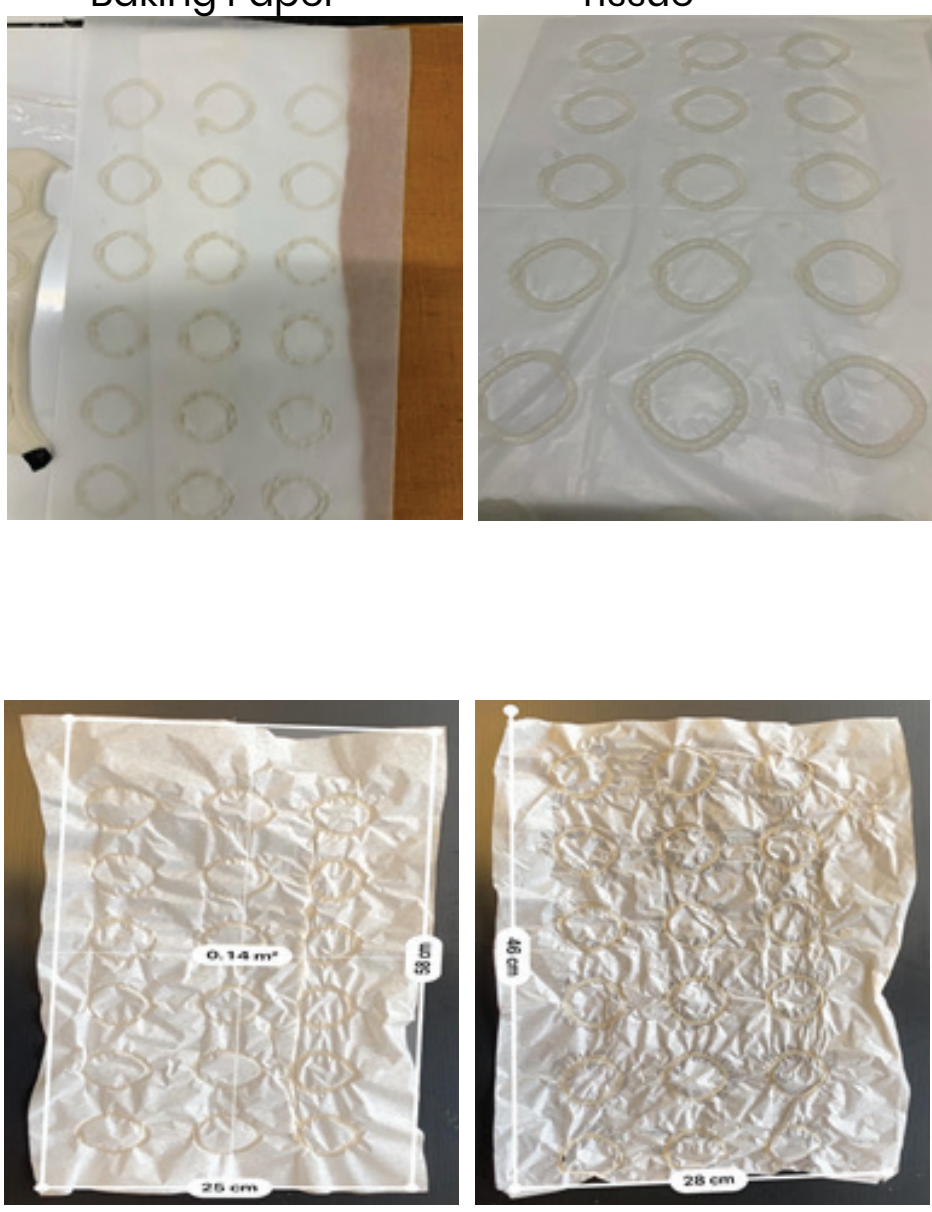


\section{EXTRUSION PATTERN NUMBER 2}

Selected substrates:

Baking Paper, Calico, Cotton, Jute, Muslin, Cotton + Muslin

FIGURE 4.12B

Developed Grasshopper Script

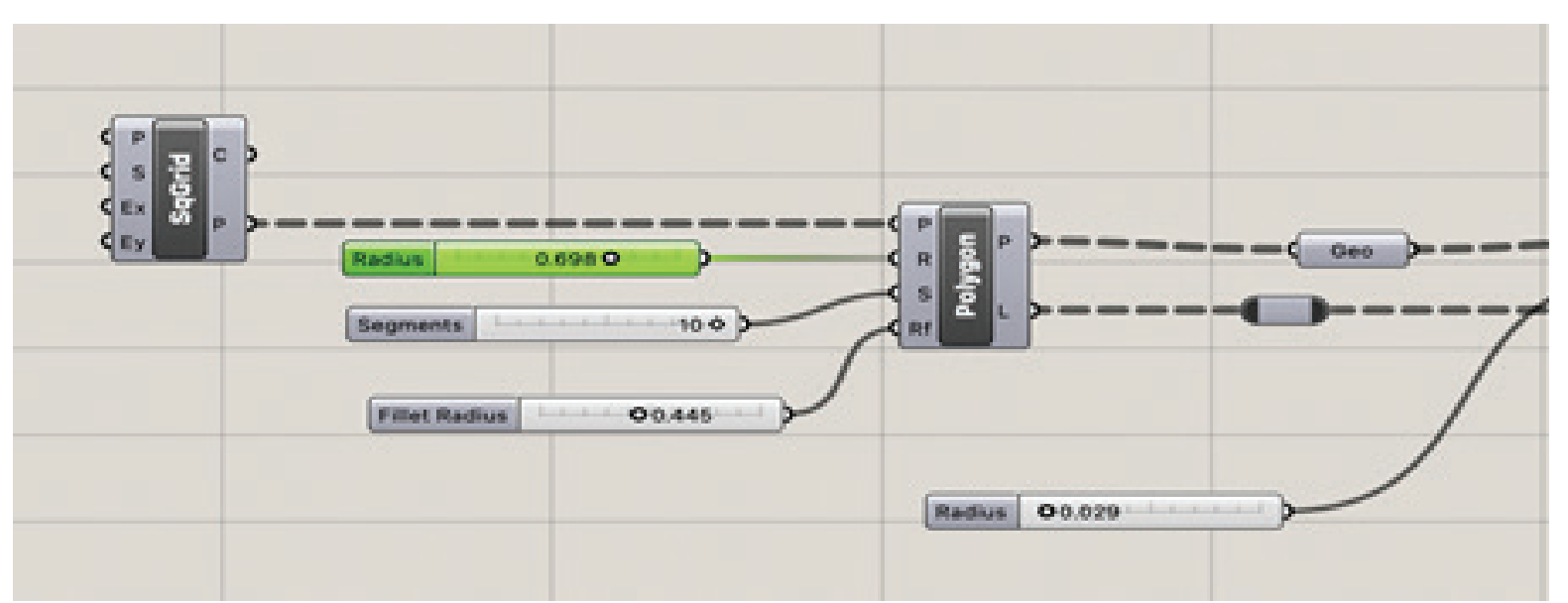

Source: Author's work (2021)
FIGURE 4.12A

\section{Extrusion Pattern}

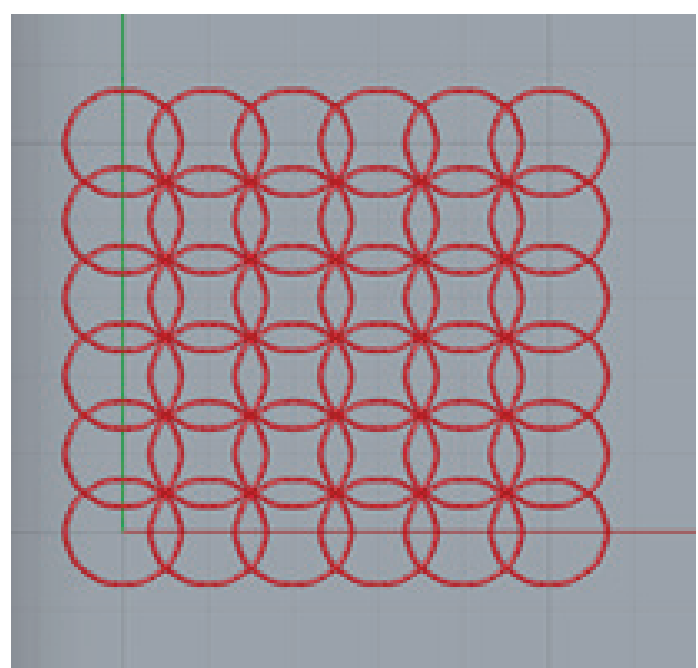

Source: Author's work (2021) 
FIGURE 4.12C

Observations of the Second Pattern on Different Substrates Upon Extrusion

Substrate:

Baking Paper

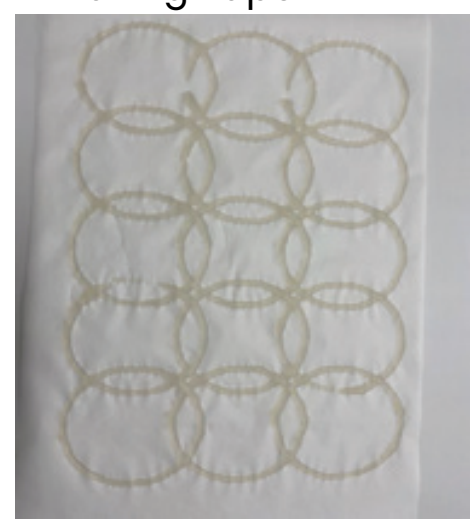

\section{Before drying}
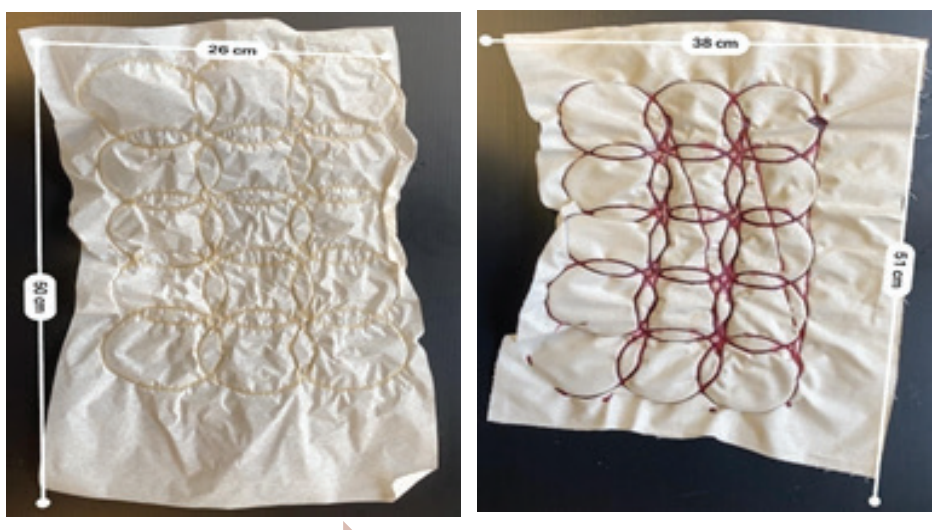

After drying

Calico

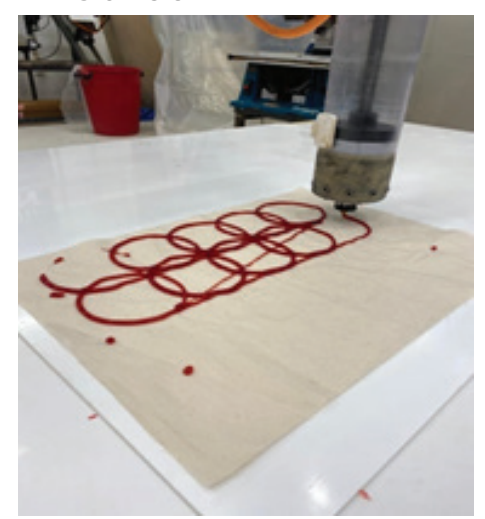

Cotton
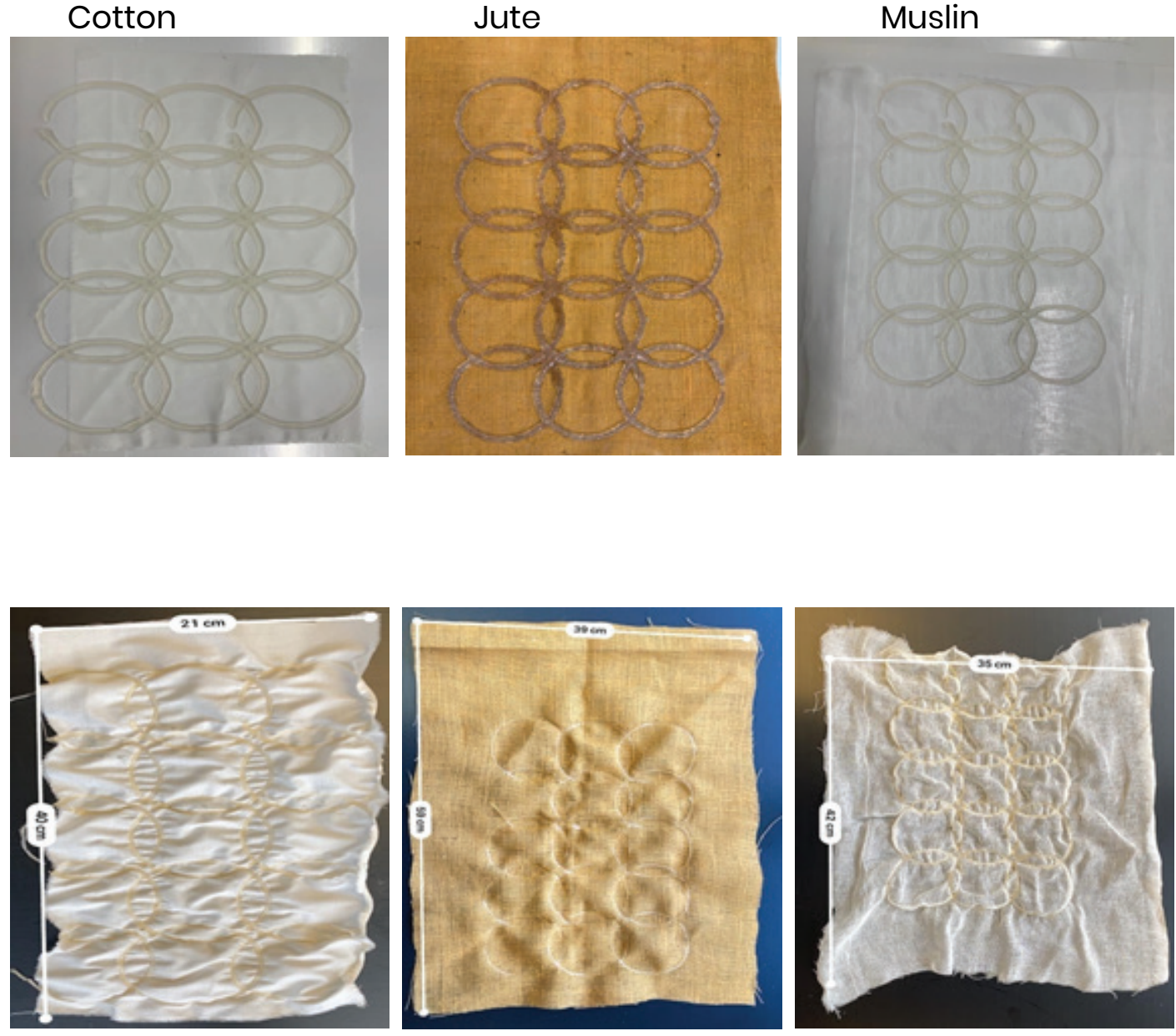
Substrate: Muslin + Cotton

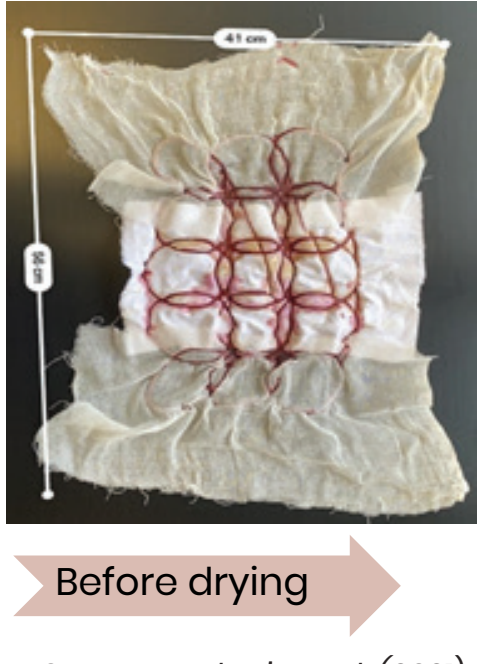

Source: Author's work (2021)

FIGURE 4.13B

Developed Grasshopper Script

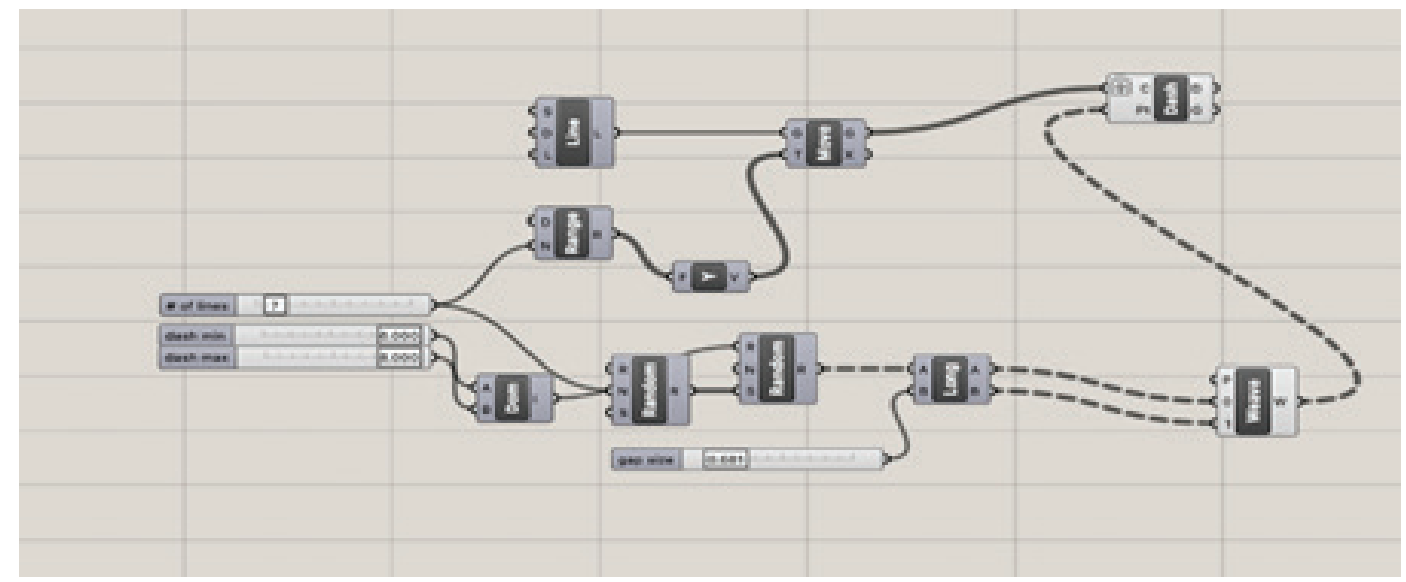

Source: Author's work (2021)

\section{EXTRUSION PATTERN NUMBER 3}

Selected substrates:

Calico, Muslin
Extrusion Pattern

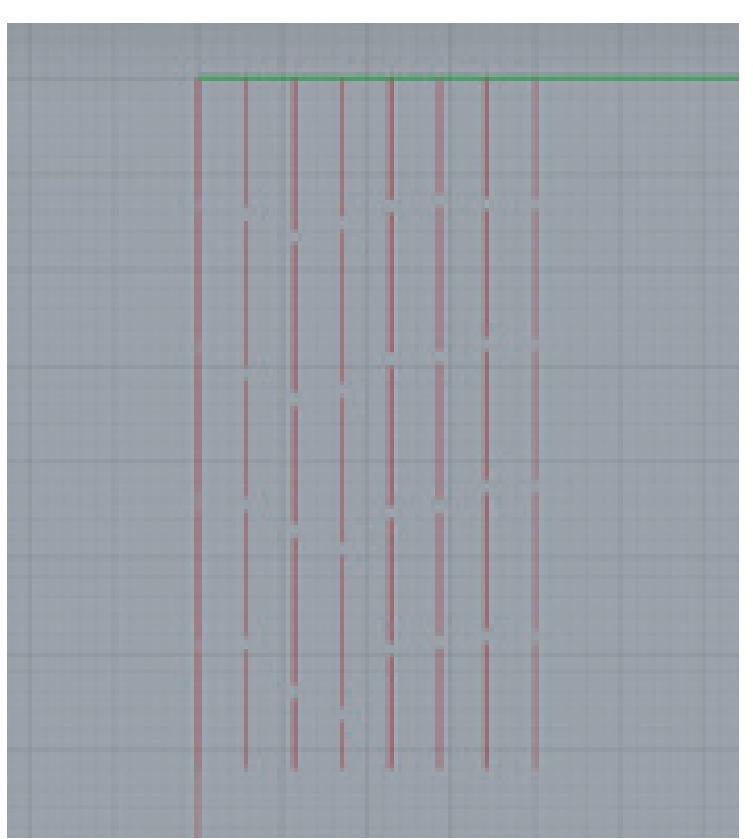

Source: Author's work (2021) 
FIGURE 4.13C

Observations of the Third Pattern on Different Substrates Upon Extrusion

\section{Substrate:}

Calico

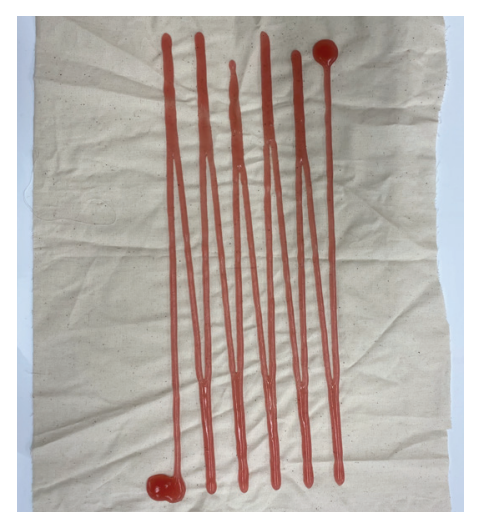

Muslin

Before drying

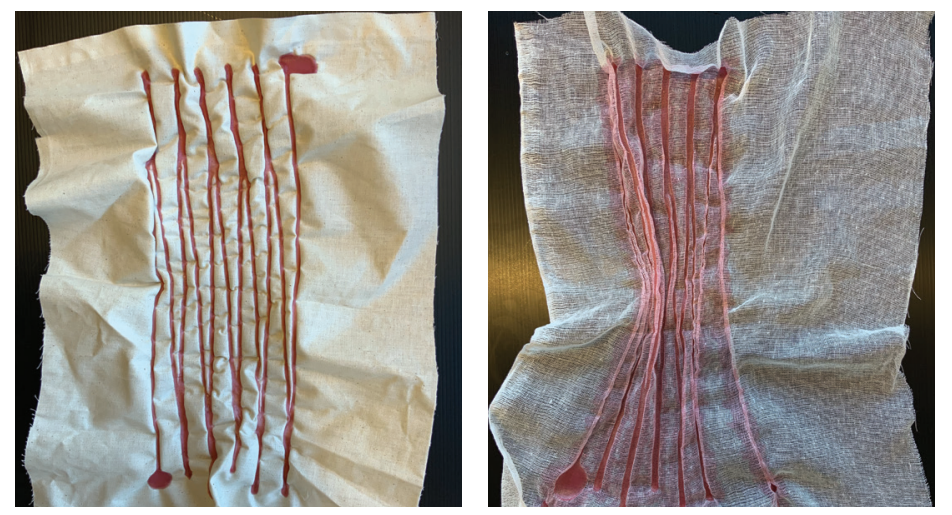

After drying

Source: Author's work (2021) 


\section{EXTRUSION PATTERN NUMBER 4}

Selected substrates:

Calico, Cotton, Muslin

FIGURE 4.14A

Extrusion Pattern

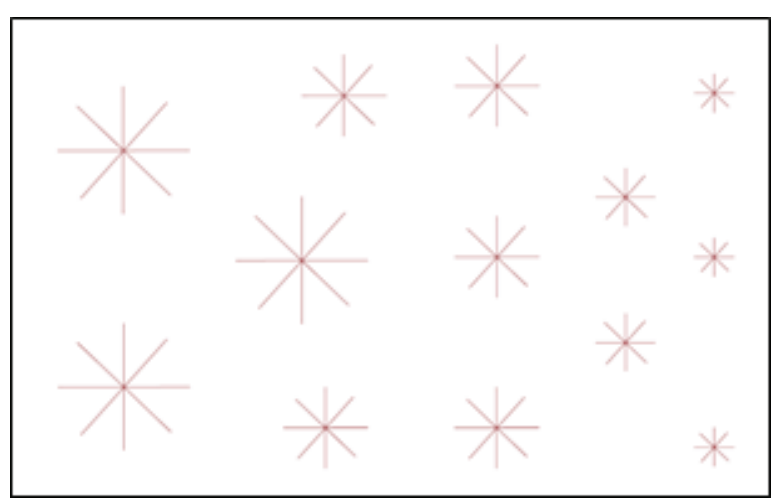

Source: Author's work (2021)

FIGURE 4.14B

Developed Grasshopper Script

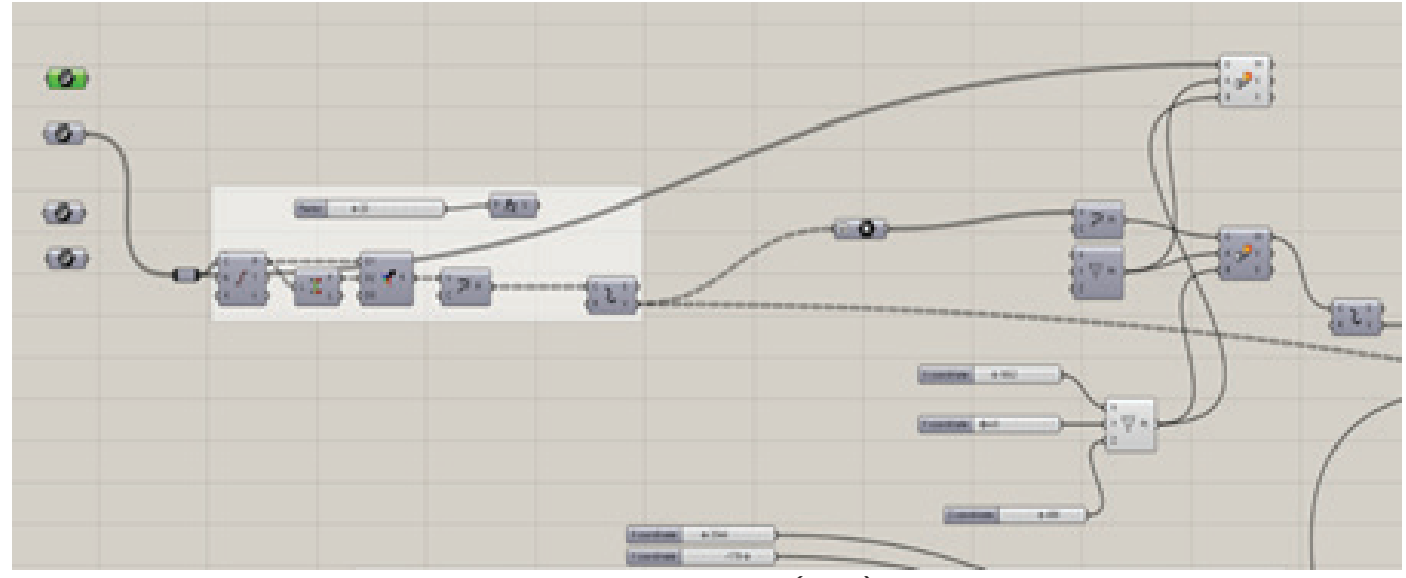

Source: Author's work (2021)

FIGURE 4.14C

Observations of the Fourth Pattern on Different Substrates Upon Extrusion

Substrate:

Calico

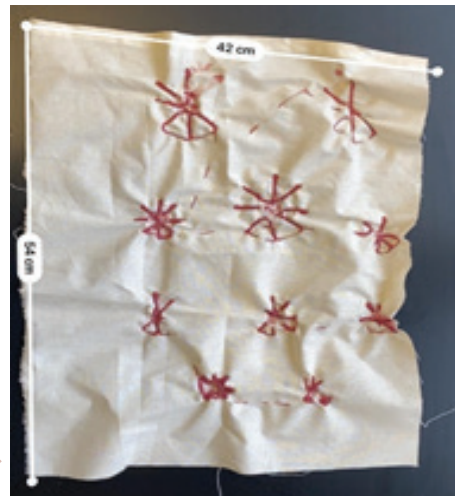

Cotton

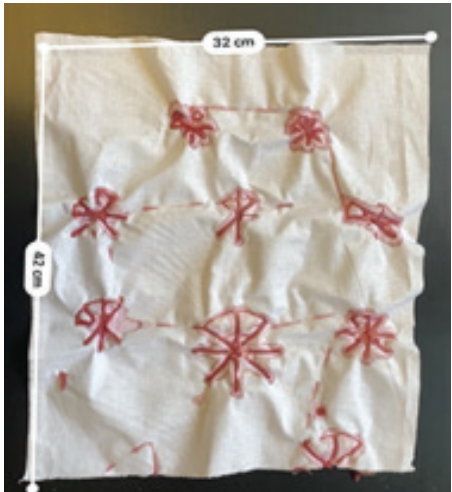

Source: Author's work (2021)
Muslin

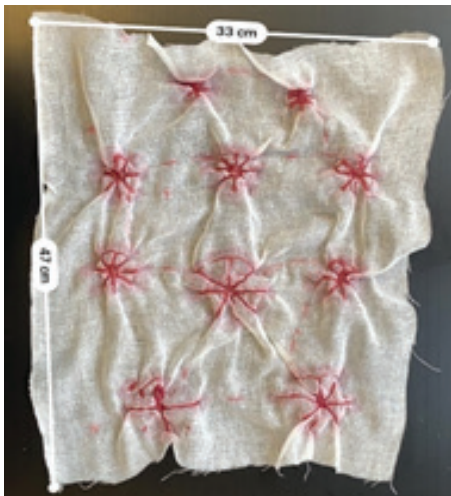




\section{EXTRUSION PATTERN NUMBER 5}

Selected substrates:

Calico, Cotton, Muslin

FIGURE 4.15A

Extrusion Pattern

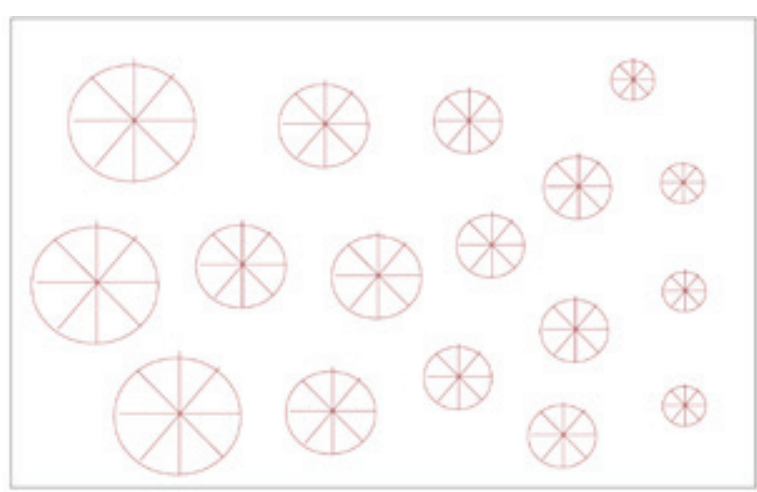

Source: Author's work (2021)

FIGURE 4.15B

Developed Grasshopper Script

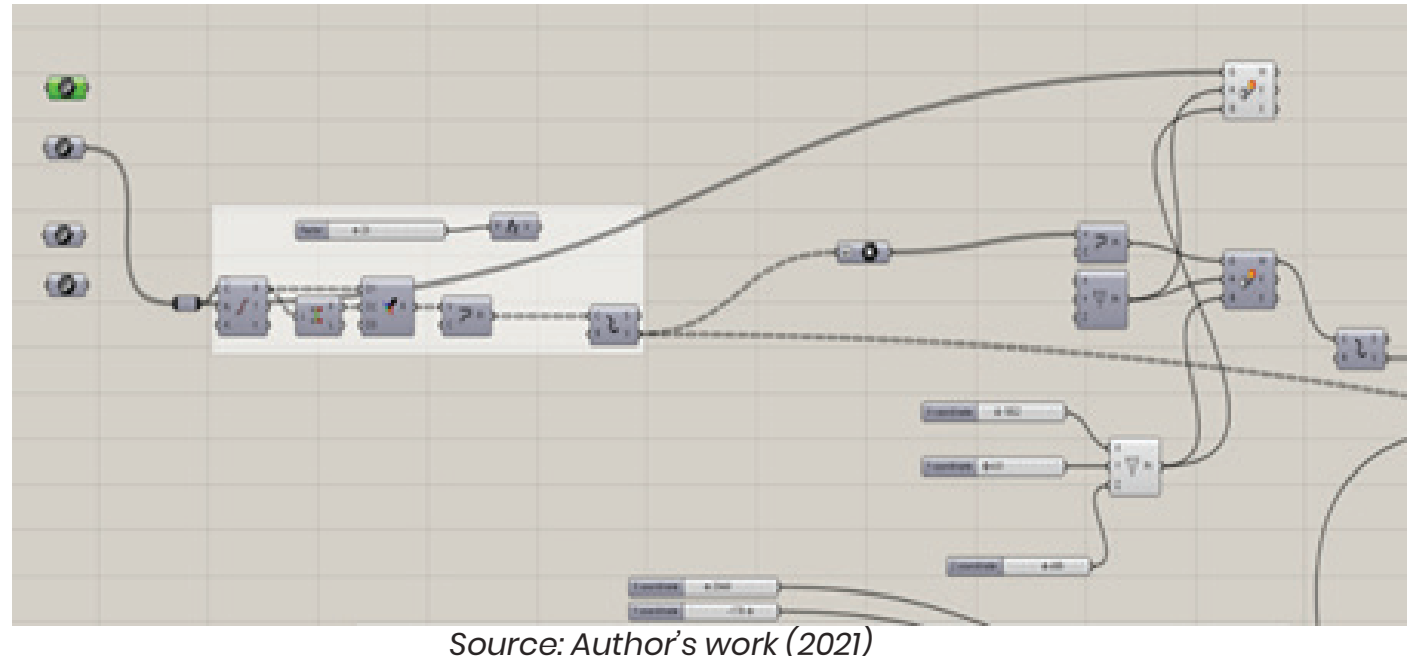

FIGURE 4.15C

Observations of the Fourth Pattern on Different Substrates Upon Extrusion

Substrate:

Calico

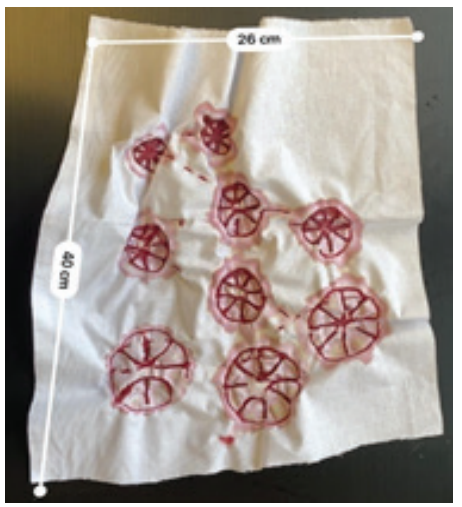

Cotton

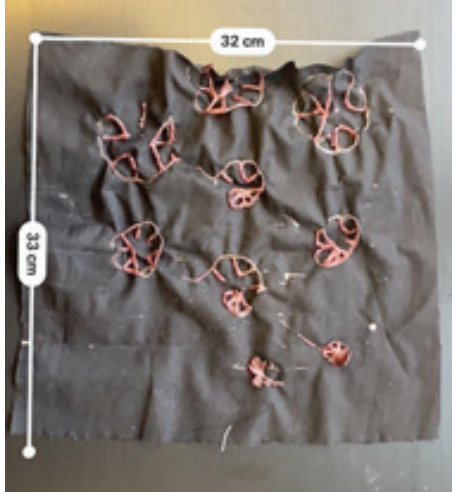

Source: Author's work (2021)
Muslin

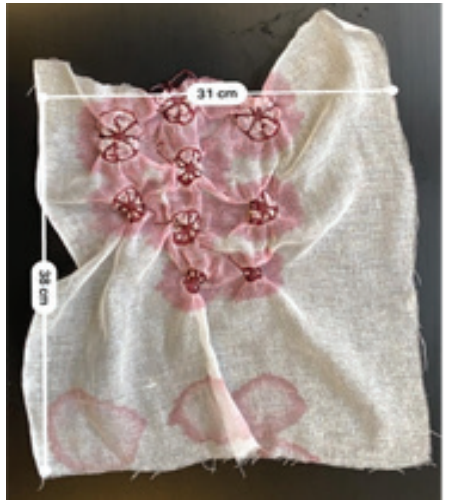




\section{Conclusion:}

The percentage of shrinkage in substrates, exhibited three days after the extrusion of the printable agar medium was varied depending on the type of substrate used. Muslin recorded the highest shrinkage value in all patterns. However, the area density of the extruded patterns also had a major contribution towards the observed shrinkage. The denser the pattern area, the higher the shrinkage percentage was.

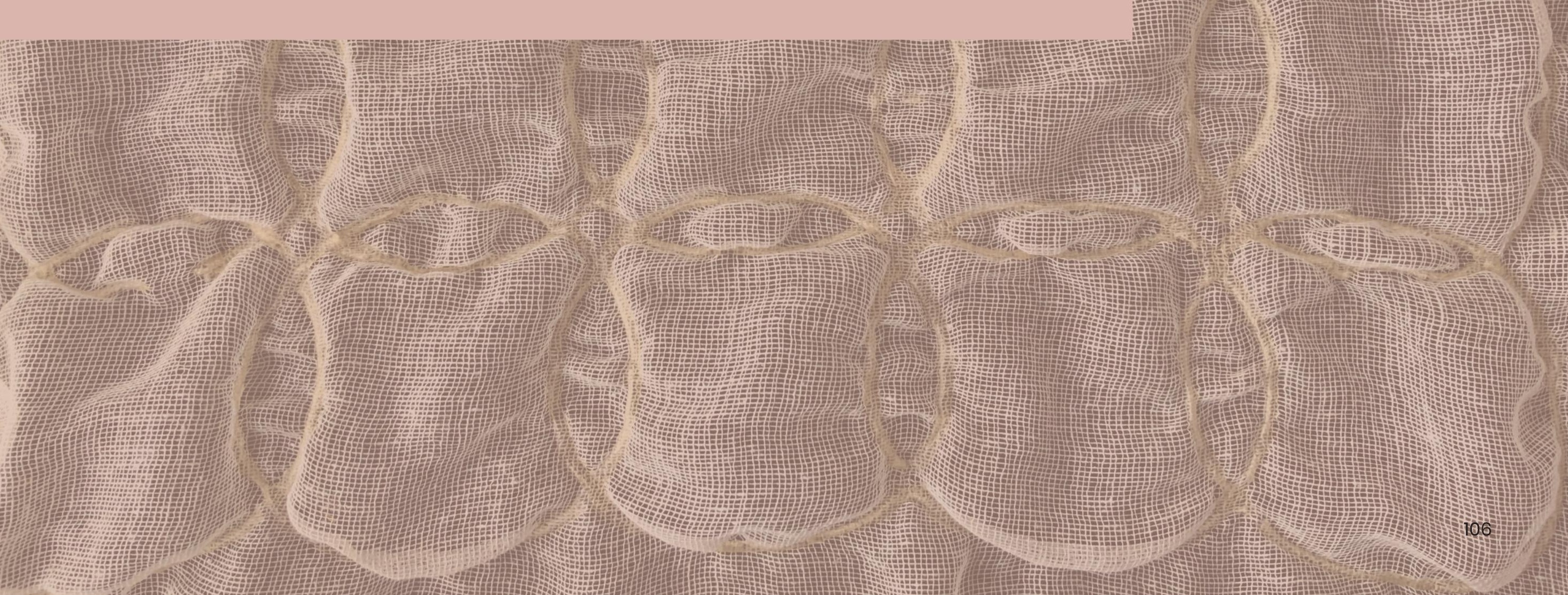




\section{MIPM - MYCELIUM INOCULATED PRINTABLE MEDIA}

Moving on to the experimental stage with mycelium, the same initial procedures were followed as with the first workflow experiments, while the developed printable agar medium was inoculated with mycelium before the extrusion process (Figure 4.16).

When working with mycelium, extreme care was taken to keep the extrusion substrates free of contaminants. Because of the increase in scale, the microwave sterilisation method was impracticable; all tools, substrates, and storage mediums were disinfected with isopropyl alcohol spray.

Upon extrusion, the substrates were placed inside plastic containers and kept inside a dark chamber for 14 days.

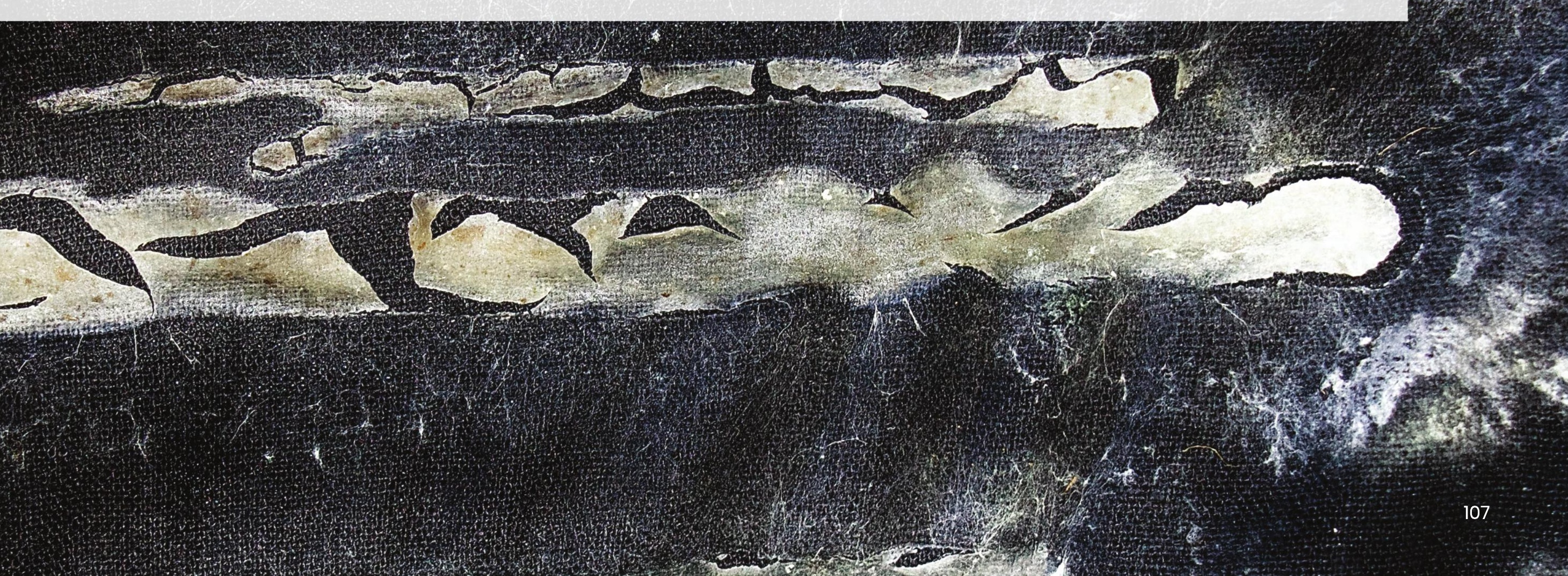




\section{FIGURE 4.16}

Workflow Diagram of Digital Extrusion

Exercises Conducted Using the

Mycelium Inoculated Printable Agar Medium on Paper and Fabric Surfaces

Prepare the printable agar medium

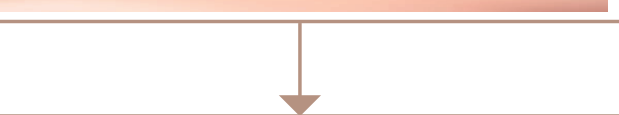

Inoculate with mycelium

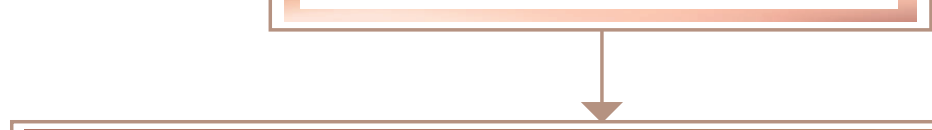

Sterilize all equipment using isopropyl alcohol spray and pour the paste into a stylized bag

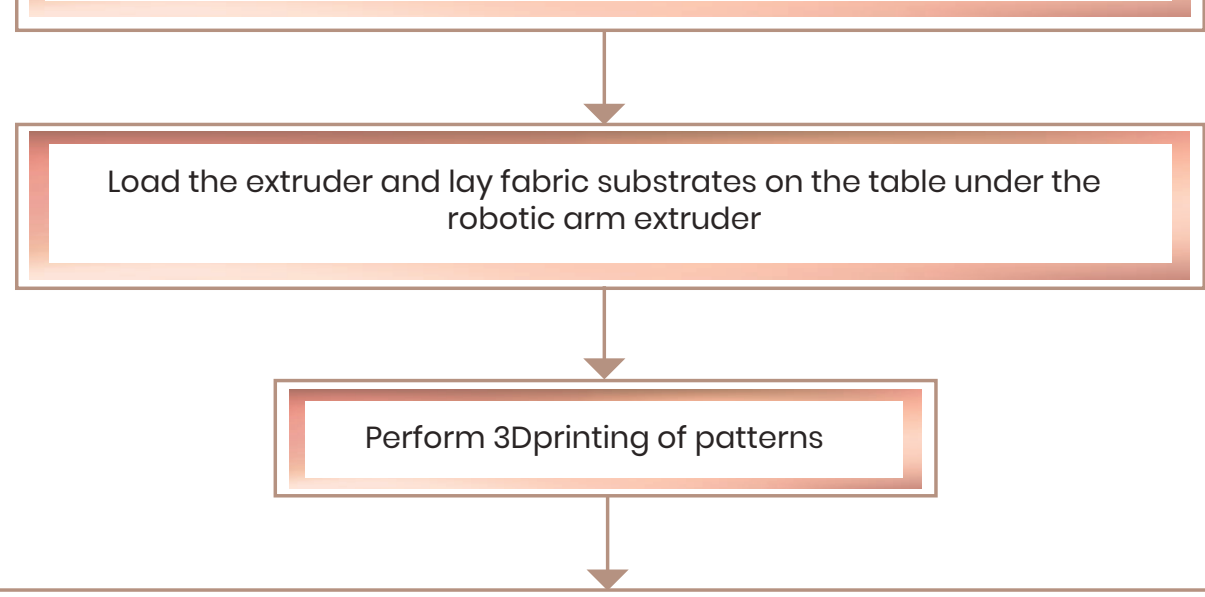

Soon after the completion of printing each pattern, place the fabrics inside a sealable plastic container with an air filter

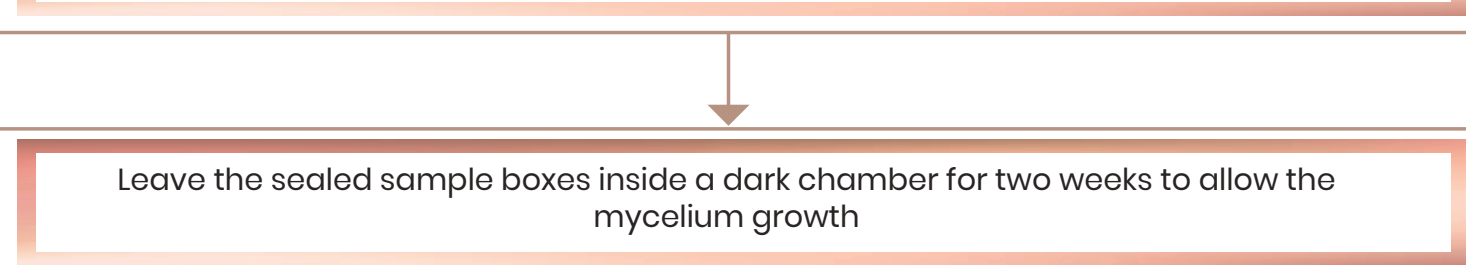

Source: Author's work (2021) 
The objective of this set of experiments was to determine how much the fabric substrates would shrink upon drying when the printable agar medium, inoculated with mycelium, was used. Having printed a range of patterns the growth of mycelium could be observed in relation to digitally generated patterns.

Observations photographed after seven days from the extrusion, are illustrated in Figure 4.17 below.

\section{FIGURE 4.17}

Growth of Mycelium in 3D Printed Patterns on Fabric Substrates, Seven Days After Extrusion

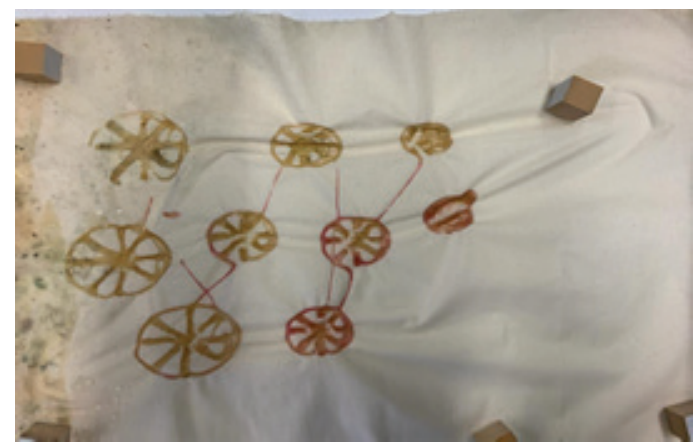

Pattern 5 on Calico

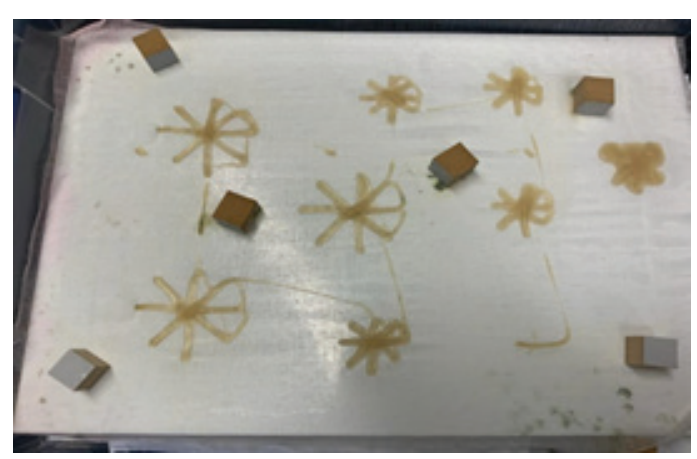

Pattern 4 on Muslin

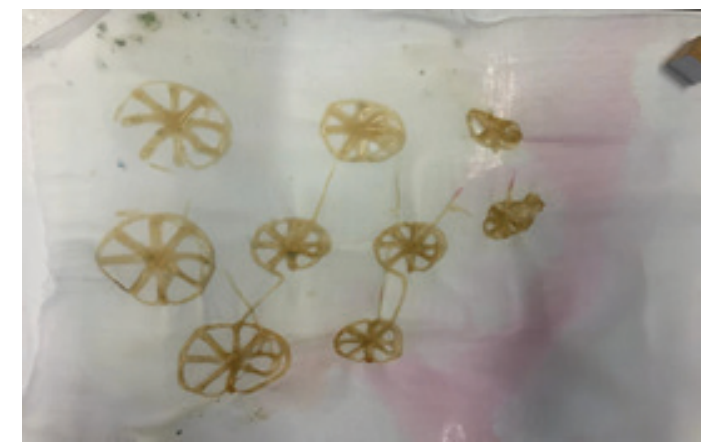

Pattern 5 on Muslin

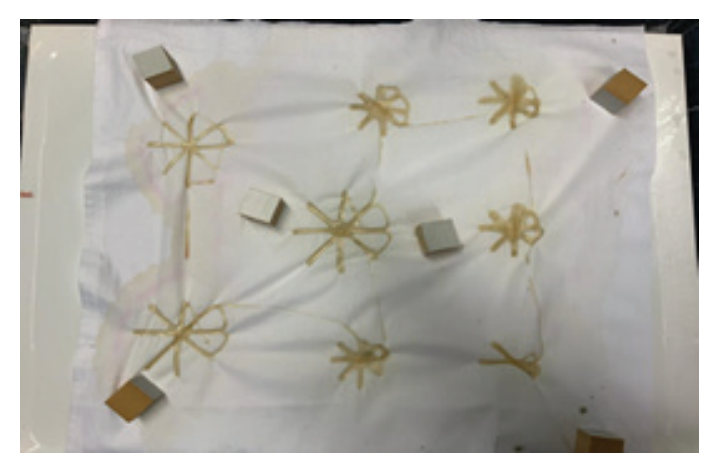

Pattern 4 on White Cotton

Source: Author's work (2021)

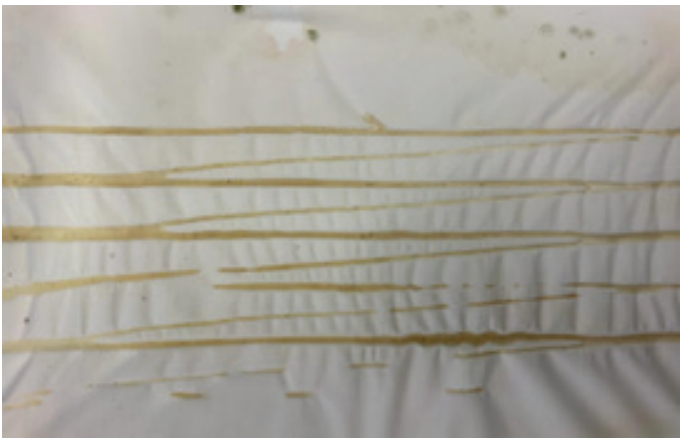

Pattern 3 on White Cotton

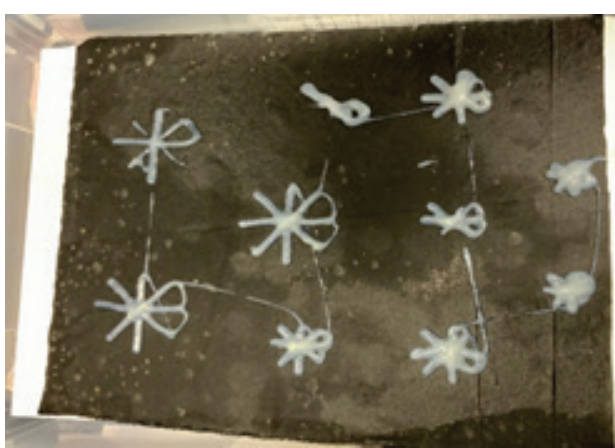

Pattern 4 on Muslin

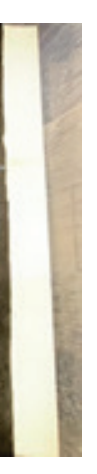


Observations recorded after fourteen days from the date of extrusion, are shown in Figure 4.18 below.

FIGURE 4.18

Growth of Mycelium in 3D Printed Patterns on Fabric Substrates, 14 Days After Extrusion

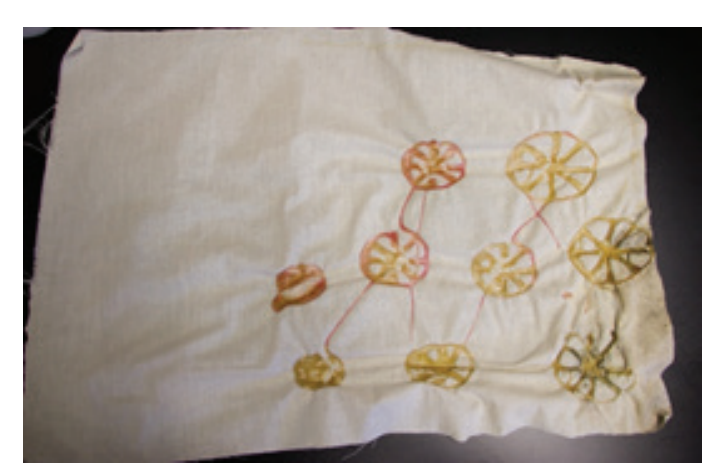

Pattern 5 on Calico

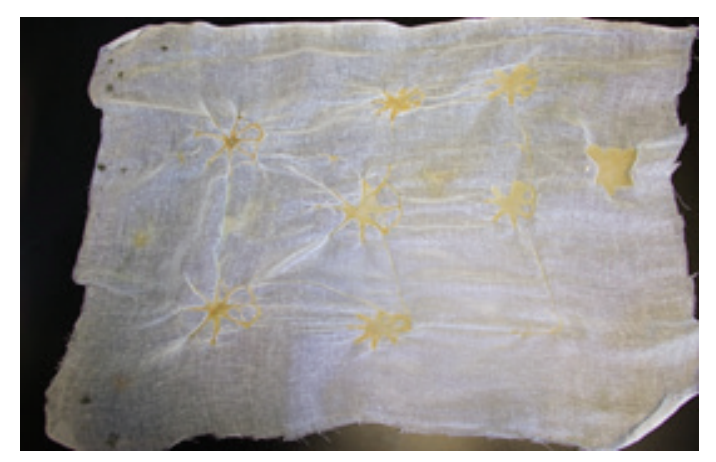

Pattern 4 on Muslin

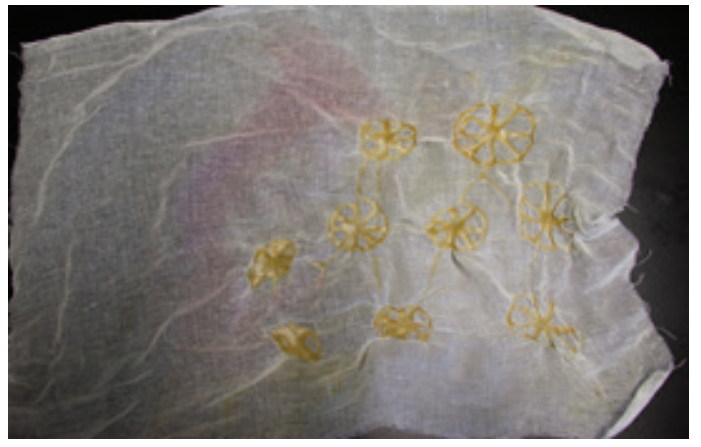

Pattern 5 on Muslin

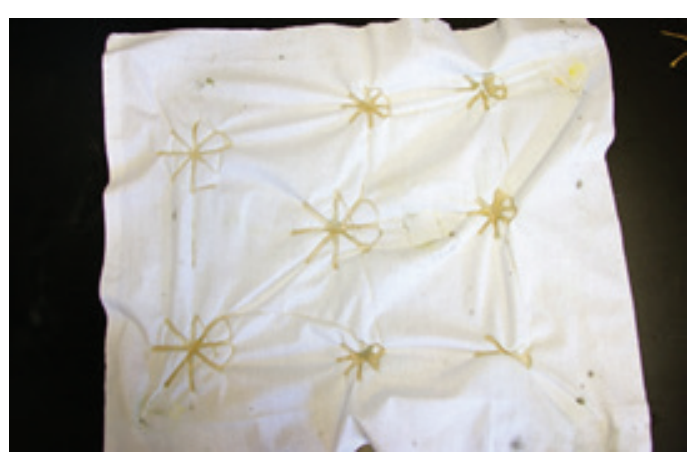

Pattern 4 on White Cotton

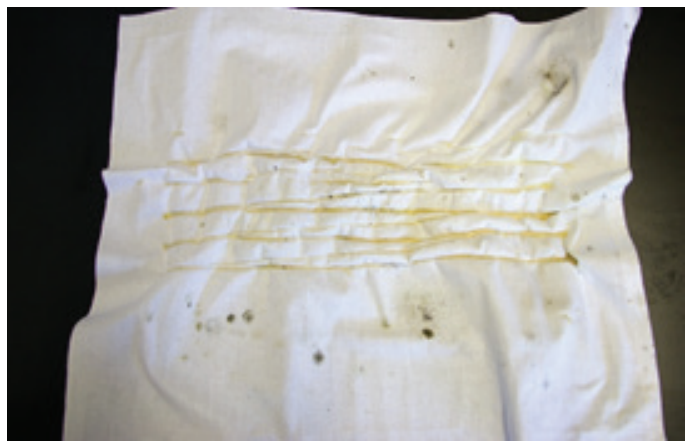

Pattern 3 on White Cotton

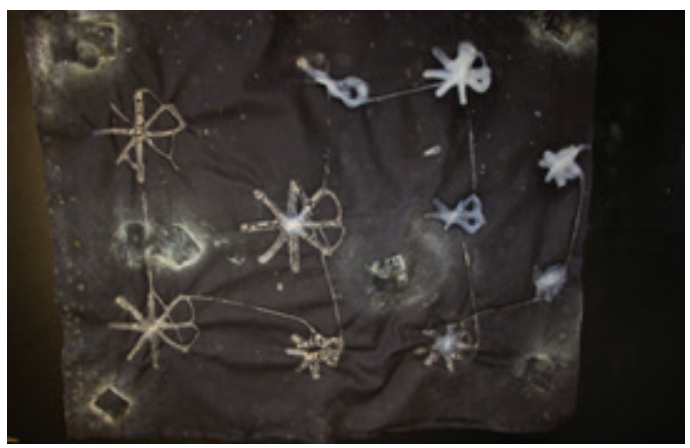

Pattern 4 on Muslin

Source: Author's work (2021)

After fourteen days, the samples were taken out from the containers and allowed to dry to gain the natural shrinkage forms.

Here, it was observed that the shrinkage extents of mycelium inoculated patterns were very much similar to the ones extruded using only the semi-liquid culture. 
FURTHER PAPM EXPERIMENTS ON NATURAL COLOURS

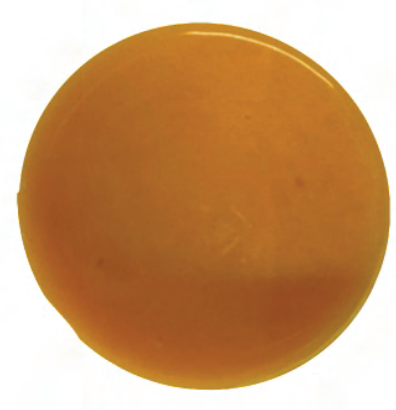

Turmeric

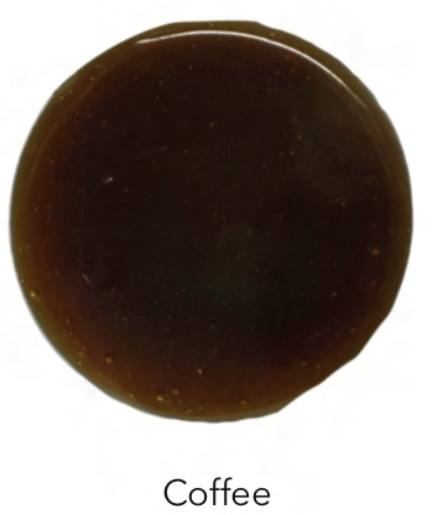

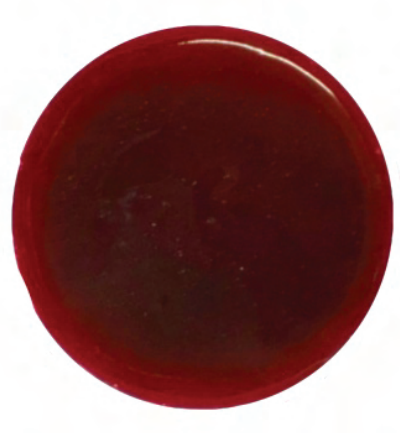

Beetroot

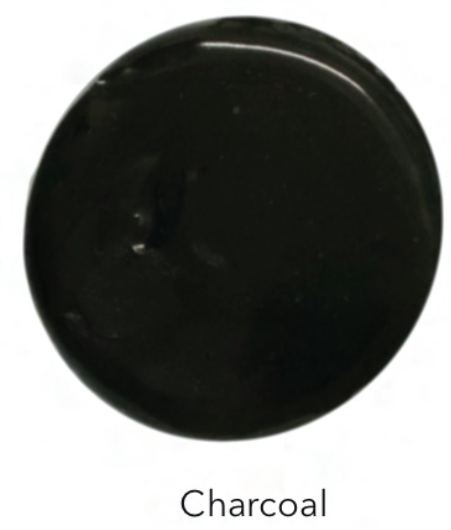

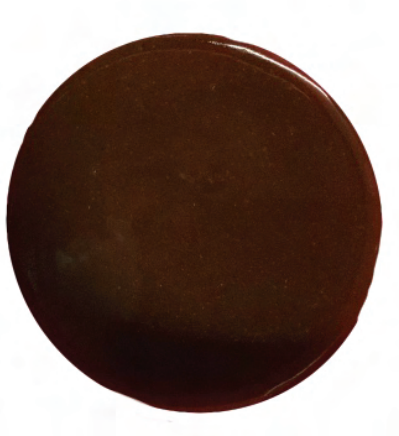

Cinnamon

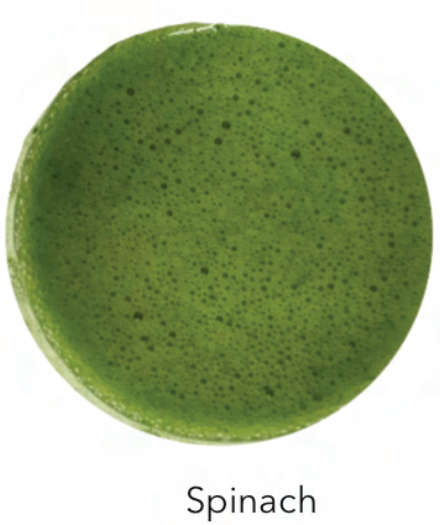


As this colour addition was successful, it was decided to experiment on more colour variants by extruding them in line patterns onto muslin and poplin fabric substrates (Figure 4.19). Here, the behaviour of natural colours from charcoal, spinach, coffee, cinnamon, saffron, and beetroot were experimented with.

Figure 4.19

Introduction of Natural Colours into the Printable Agar Medium

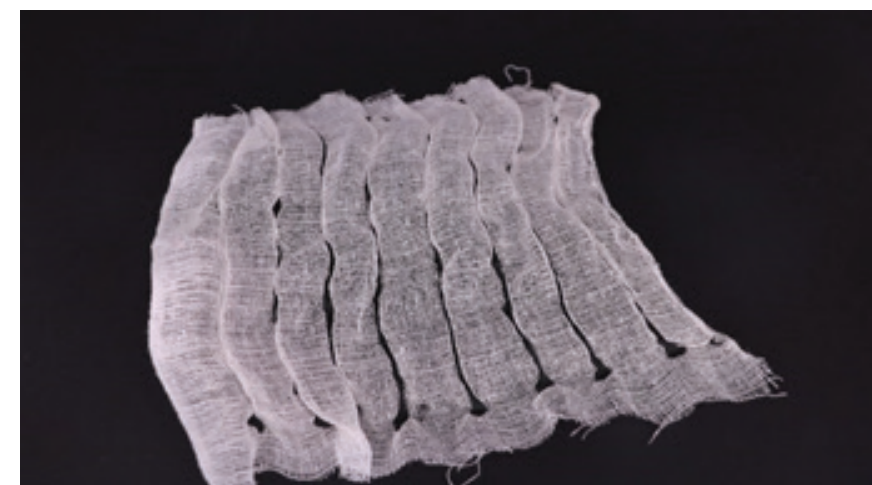

Charcoal Black patterns on Muslin

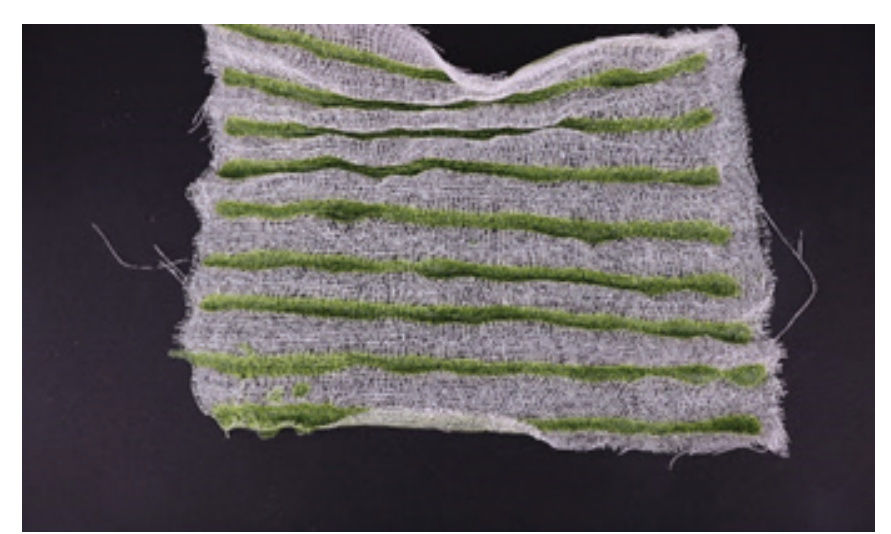

Spinatch Green patterns on Muslin

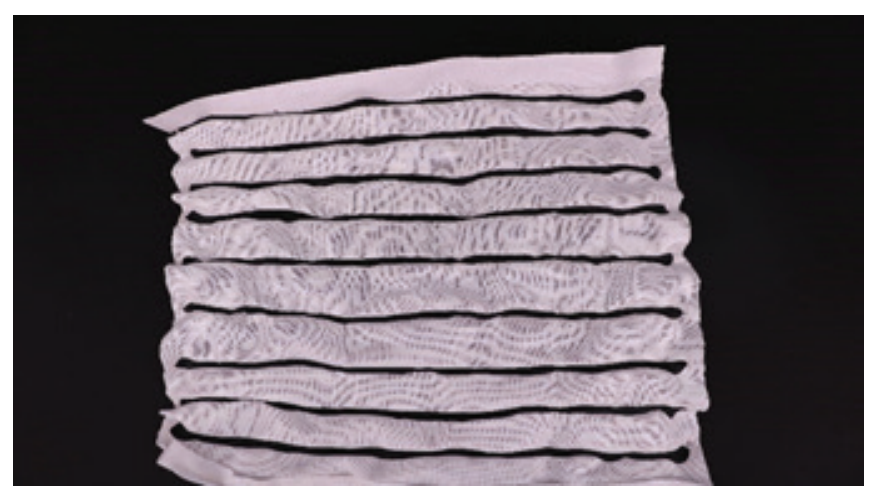

Charcoal Black patterns on Cotton

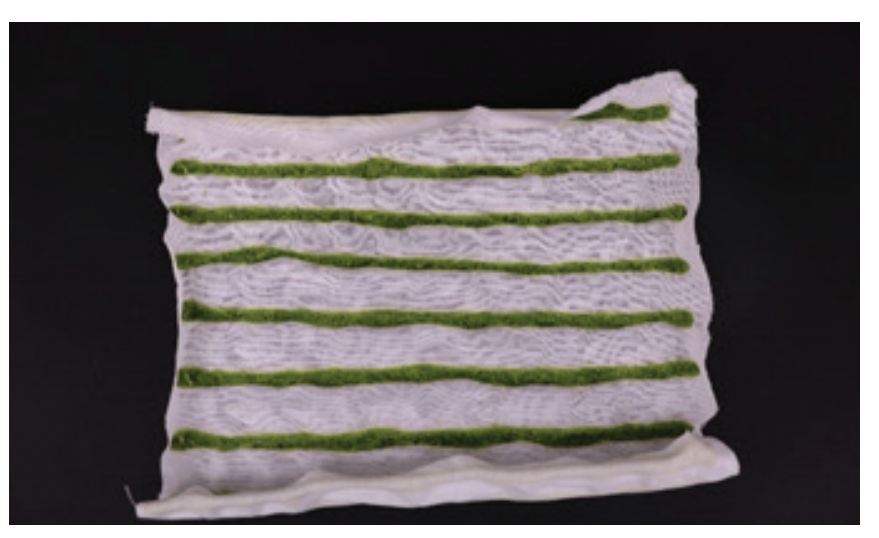

Spinatch Green patterns on Cotton 


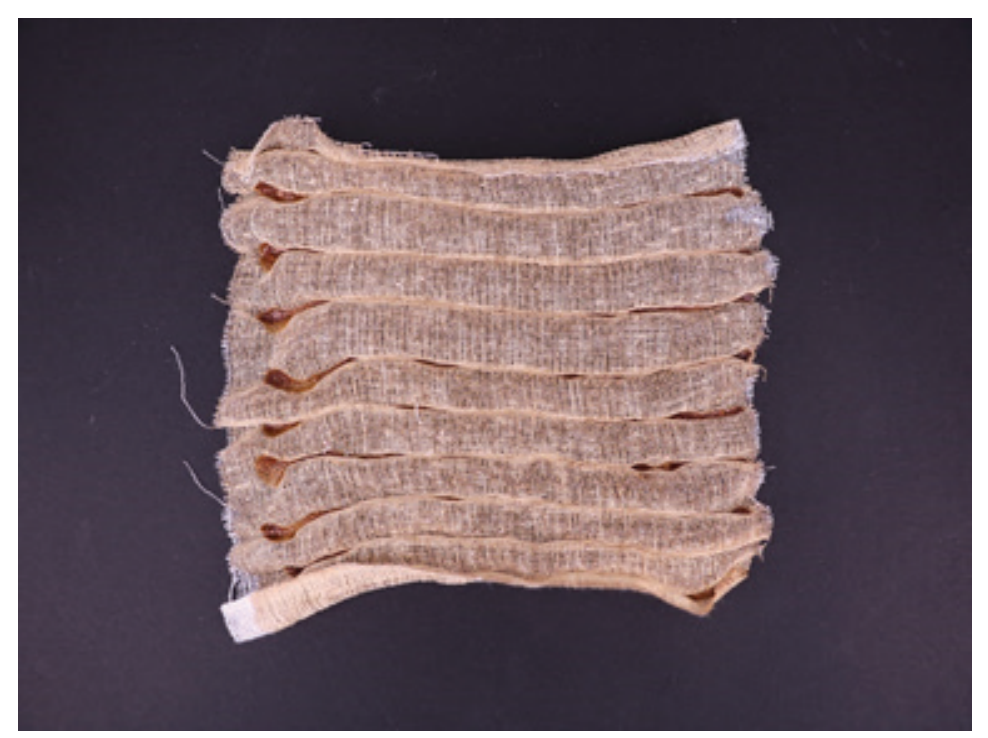

Coffee Brown patterns on Muslin

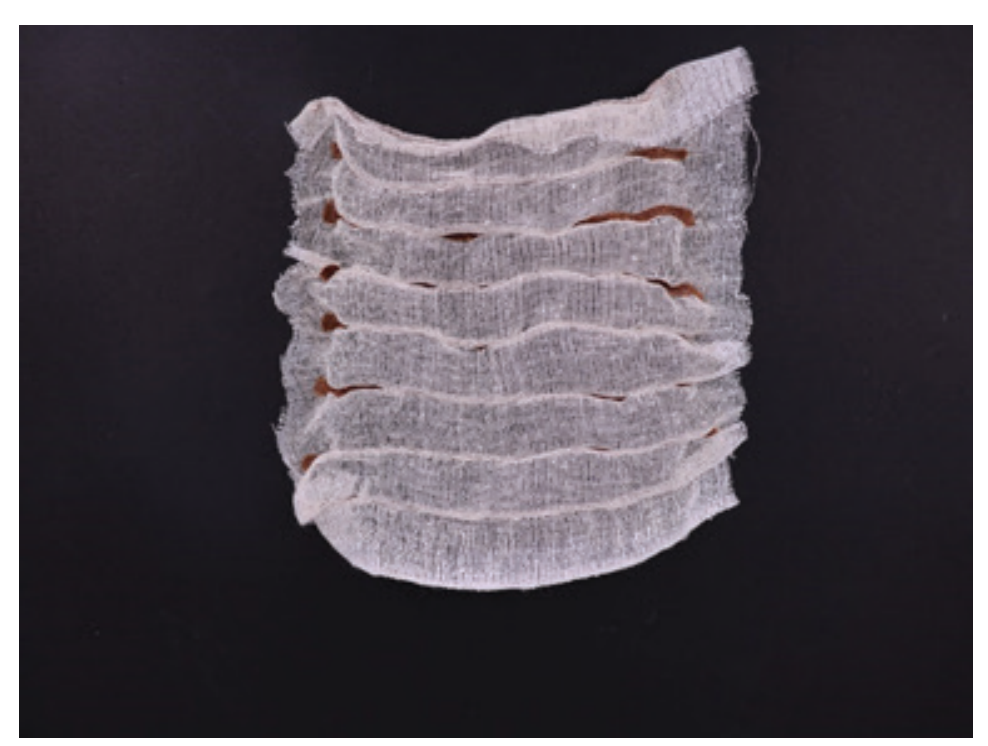

Cinnamon Tan patterns on Muslin

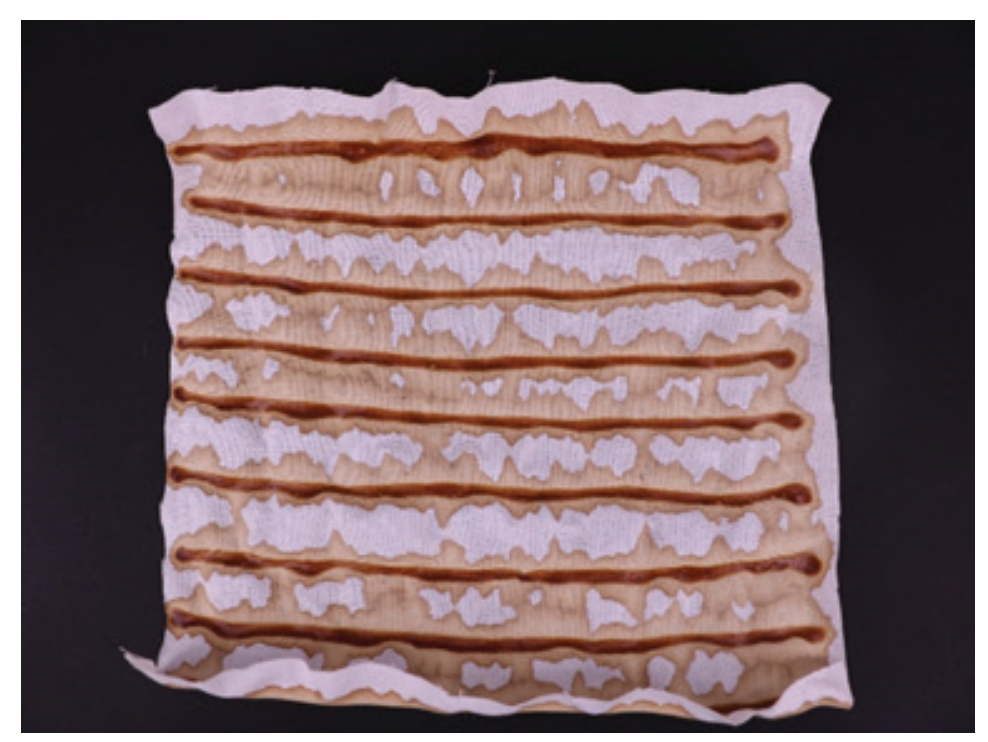

Coffee Brown patterns on Cotton

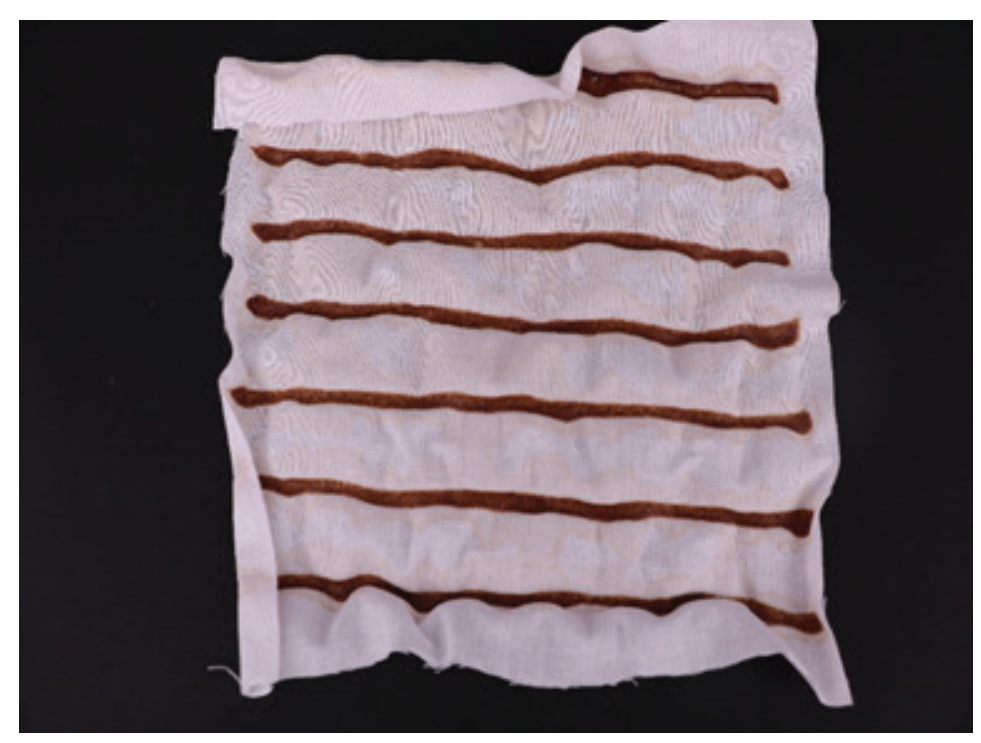

Cinnamon Tan patterns on Cotton 


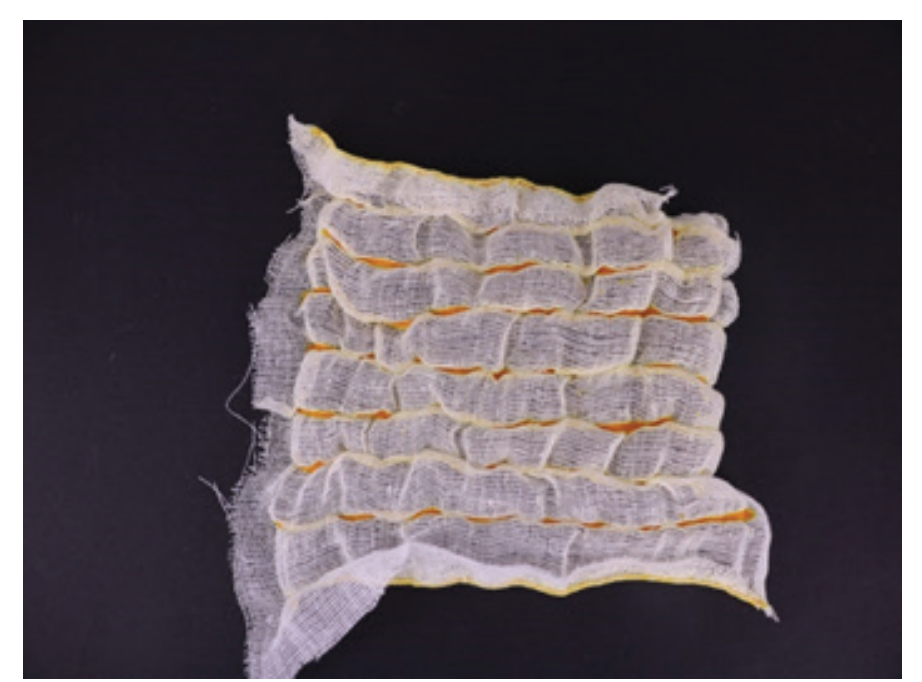

Saffron Yellow patterns on Muslin

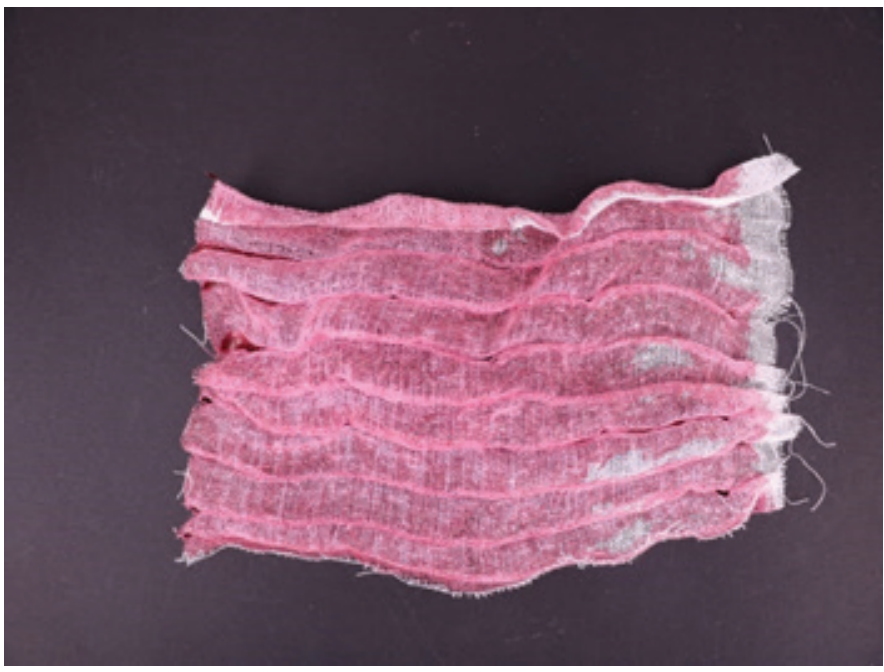

Beetroot Red patterns on Muslin

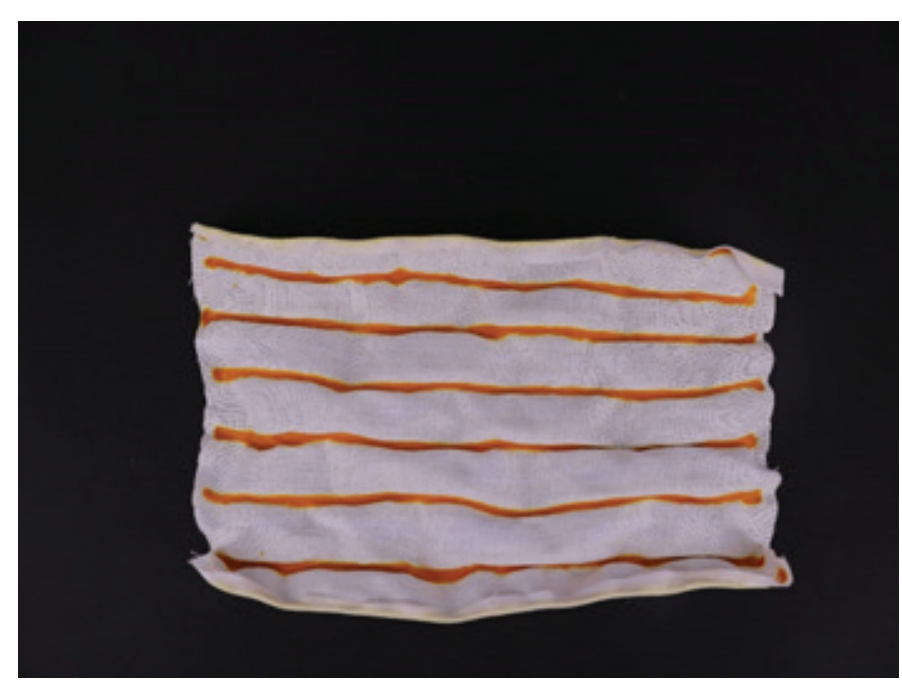

Saffron Yellow patterns on Cotton

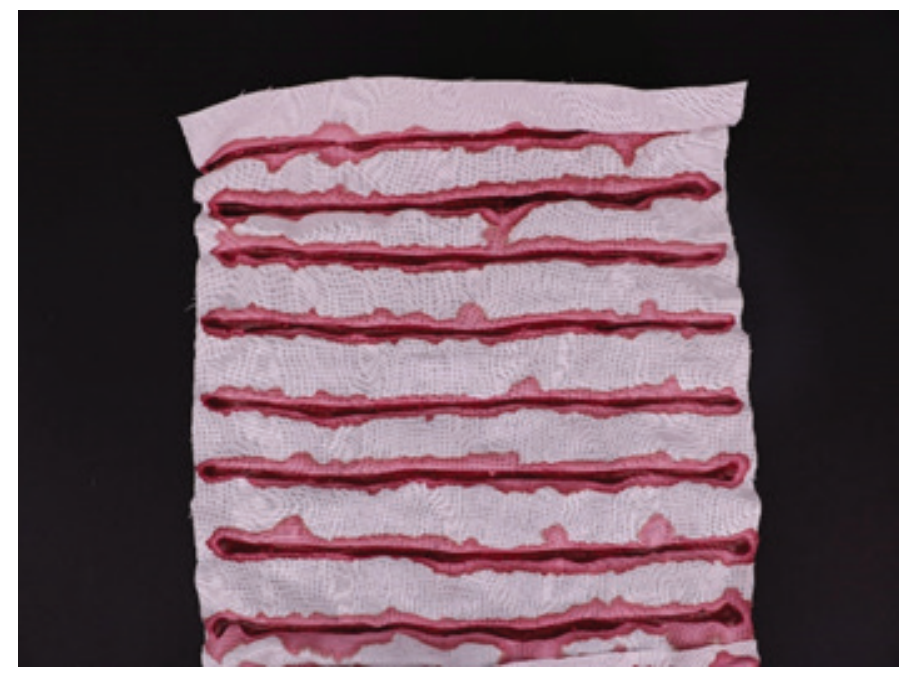

Beetroot Red patterns on Cotton 


\section{EXPERIMENTS ON SHRINKAGE ANALYSIS}

To get the shrinkage results verified scientifically, another set of experiments were planned and conducted to measure and compare the shrinkage percentages by extruding the printable agar in line patterns of $2.5 \mathrm{~mm}$ width while varying spaces between line patterns from $10 \mathrm{~mm}$ to $35 \mathrm{~mm}$. Observed shrinkage results are presented in Figure 4.20 and compared in Table 4.1 below.

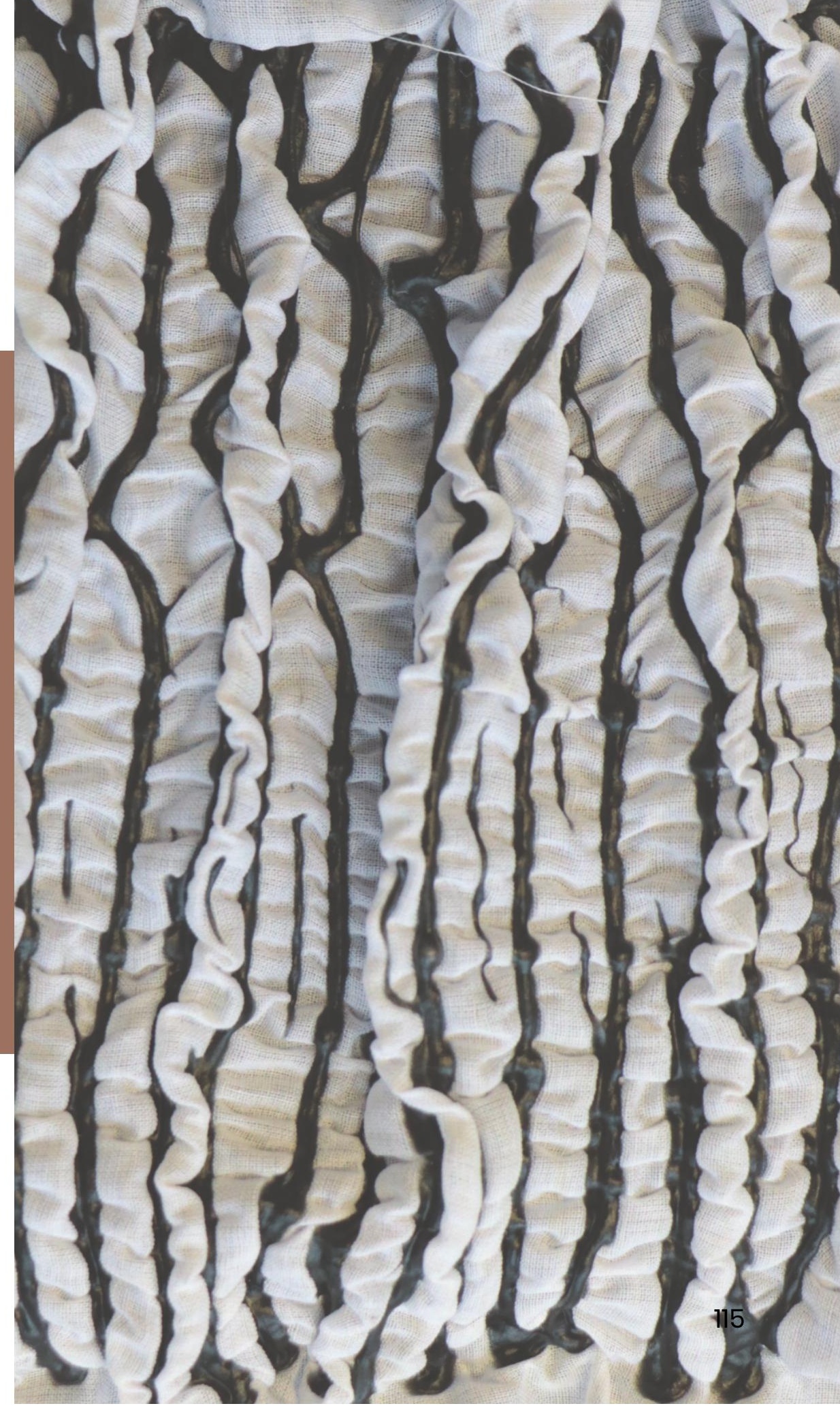


FIGURE 4.20

Shrinkage Experimental Results

Line patterns at $10 \mathrm{~mm}$ intervals

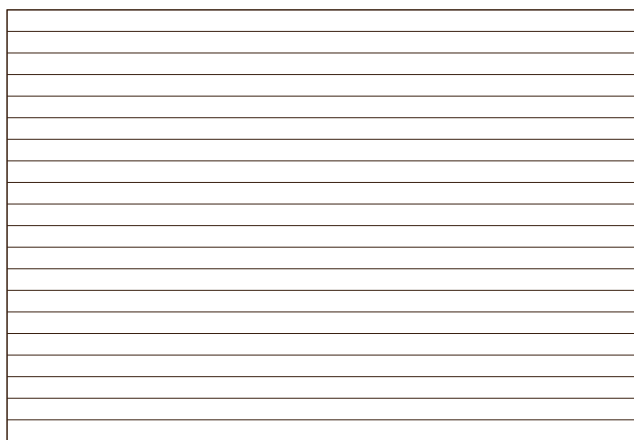

Before

Shrinkage

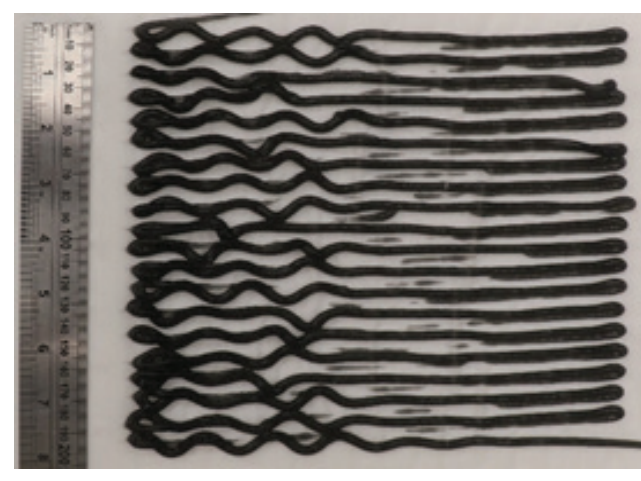

After

Shrinkage

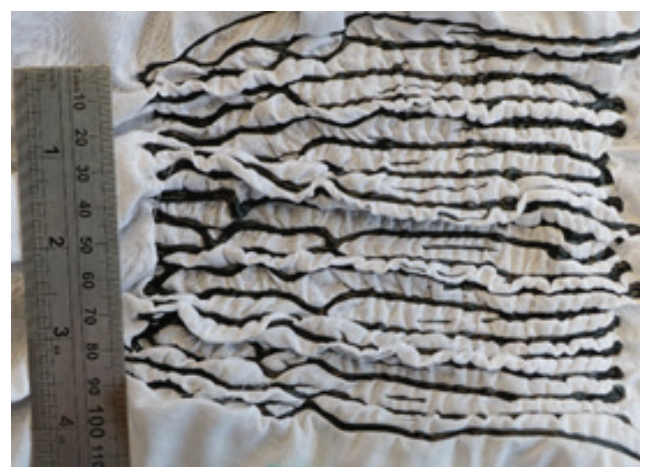

Line patterns at $15 \mathrm{~mm}$ intervals
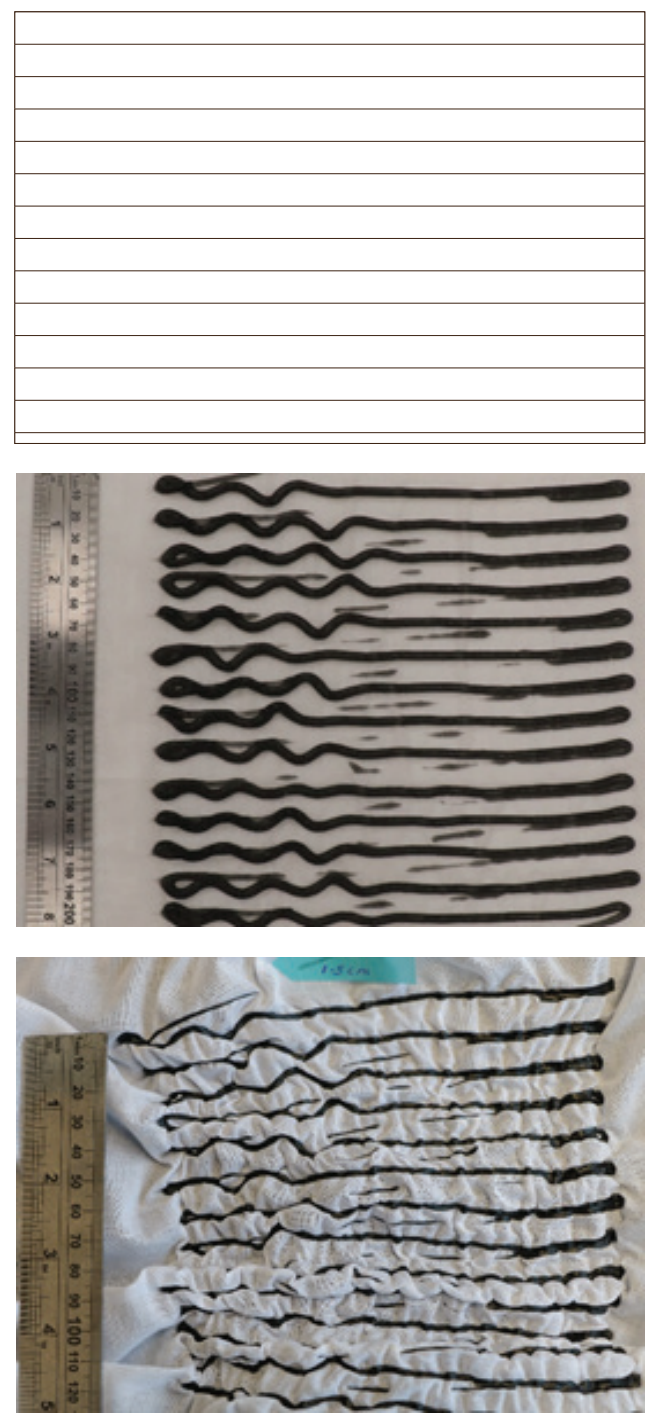

Line patterns at $20 \mathrm{~mm}$ intervals
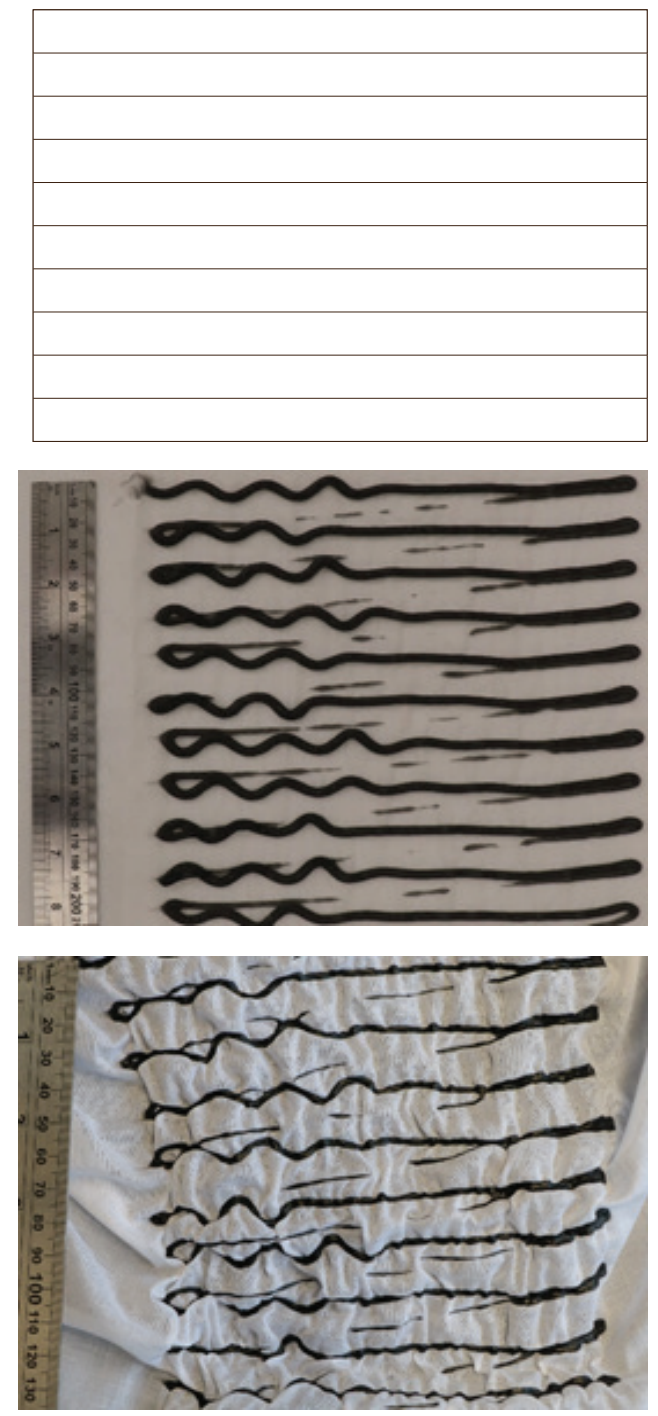
Line patterns at $25 \mathrm{~mm}$ intervals

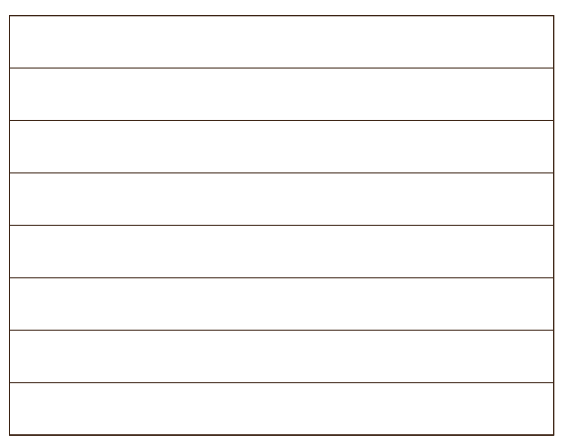

Before

Shrinkage

After

Shrinkage
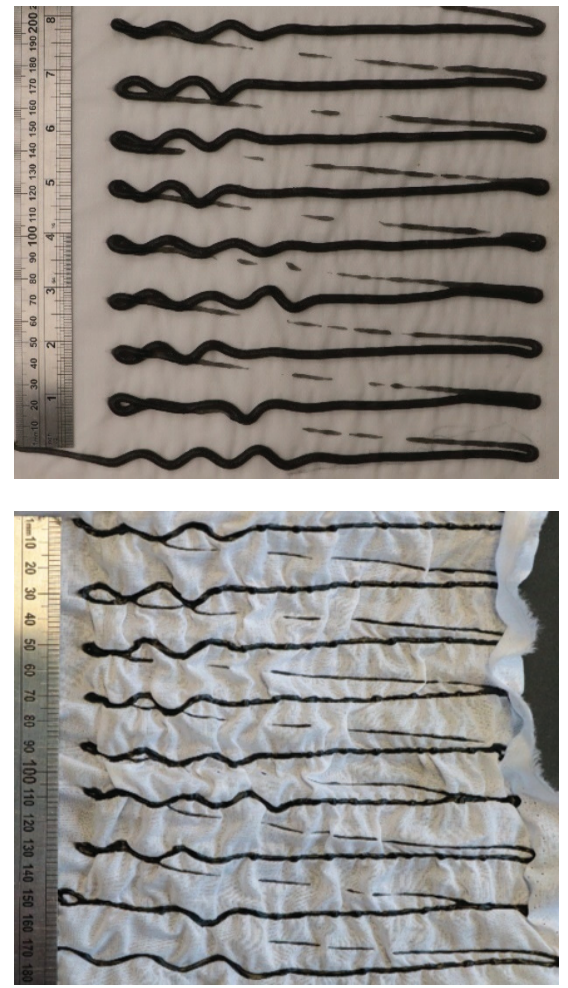

Line patterns at $30 \mathrm{~mm}$ intervals
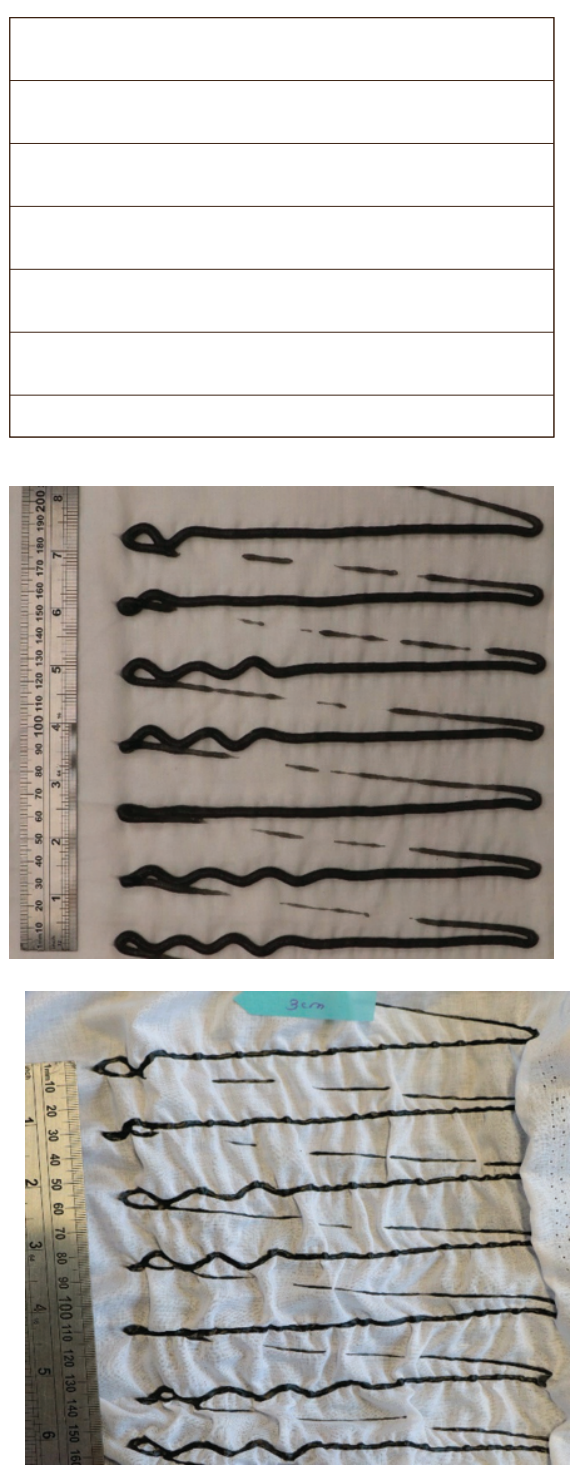

Source: Author's work (2021)
Line patterns at 35mm intervals
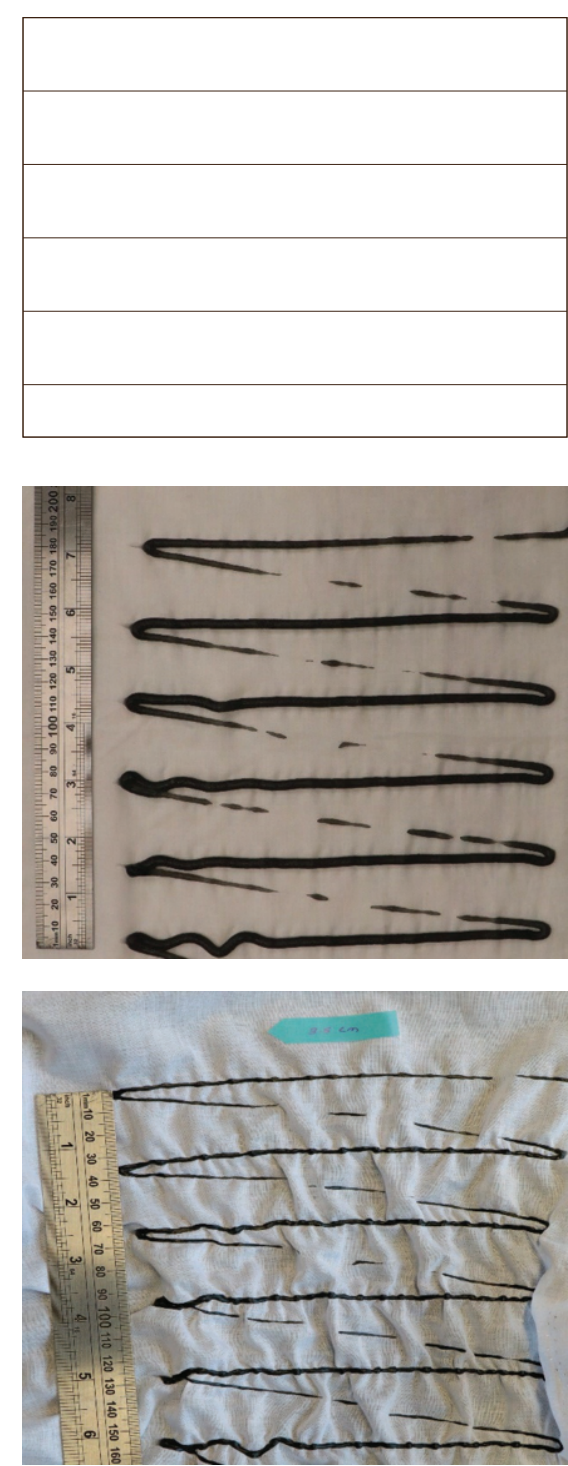
TABLE 4.1

Comparison of the Shrinkage Percentages of Line Patterns with Different Spacing

\begin{tabular}{|c|c|c|c|c|c|c|}
\hline Space between line patterns & $10 \mathrm{~mm}$ & $15 \mathrm{~mm}$ & $20 \mathrm{~mm}$ & $25 \mathrm{~mm}$ & $30 \mathrm{~mm}$ & $35 \mathrm{~mm}$ \\
\hline Pattern width before shrinkage & $200 \mathrm{~mm}$ & $200 \mathrm{~mm}$ & $200 \mathrm{~mm}$ & $200 \mathrm{~mm}$ & $200 \mathrm{~mm}$ & $200 \mathrm{~mm}$ \\
\hline Pattern width after shrinkage & $90 \mathrm{~mm}$ & $120 \mathrm{~mm}$ & $130 \mathrm{~mm}$ & $170 \mathrm{~mm}$ & $160 \mathrm{~mm}$ & $160 \mathrm{~mm}$ \\
\hline Shingake percentage & $55 \%$ & $40 \%$ & $35 \%$ & $15 \%$ & $20 \%$ & $20 \%$ \\
\hline
\end{tabular}

250

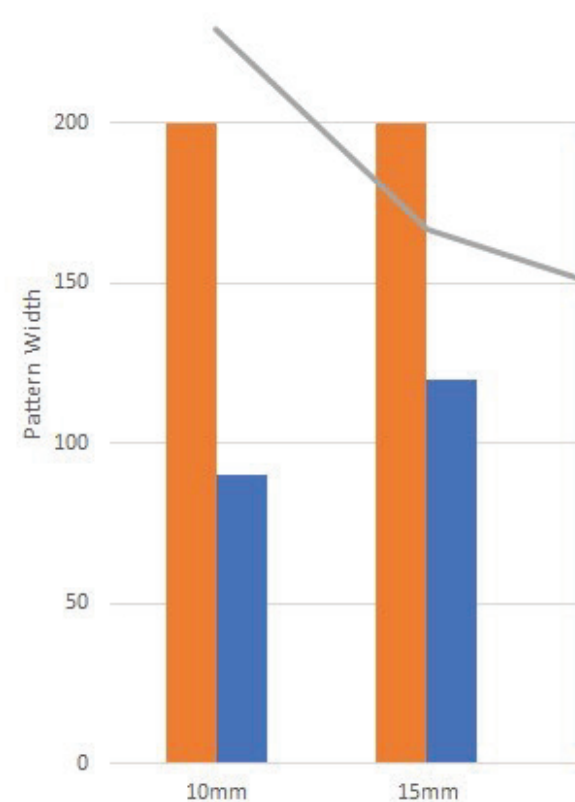

Pattern width before shrinkage
Shrinkage Analysis

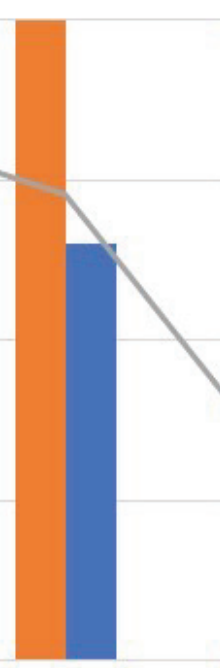

$20 \mathrm{~mm}$

Space Between Line Pattern

Pattern width after shrinkage

- Shrinkage percentage

SOURCE: AUTHOR'S WORK (2021)

$60 \%$

$50 \%$

$40 \%$

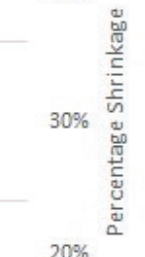

$10 \%$

$0 \%$

rertage

In evaluating the above observations and results, it became clear that more fabric shrinkage could be achieved when the line intended patterns are extruded densely on the fabric surfaces. 


\section{DESIGN IMPLICATIONS}

The analysis of shrinkage revealed a greater opportunity to apply the shrinkage forms from fabric pieces into complete fashion garments. The next chapter discusses how these shrinkage experimental results are transformed into garment forms with the adoption of digitally conceptualised designs. 
4D PARAMETRIC TAILORING FOR SUSTAINABLE HAUTE COUTURE FASHION

$\diamond$ INTRODUCTION

$\diamond$ THE WORKFLOW OF BIO-BASED 4D PRINTING FOR HAUTE COUTURE FASHION

$\diamond$ THE CONVENTIONAL WORKFLOW OF FASHION GARMENT PRODUCTION

$\diamond$ THE DIGITAL WORKFLOW

$\diamond$ FINAL DESIGNS 


\section{D PARAMETRIC TAILORING FOR SUSTAINABLE HAUTE COUTURE FASHION}

INTRODUCTION

Reflecting on previous observations, it became clear that a significant growth of mycelium could become visible over an incubation period of 14 days on the extruded culture patterns. 


\section{ELABORATION ON THE TERMINOLOGY OF 2D, 3D AND 4D PRINTING}

Usually, 3D printing refers to processes of utilizing the movement of all the three primary axes while printing. Although the extrusions were only one layer high, which is theoretically similar to $2 \mathrm{D}$ printing, throughout this thesis it is referred to as $3 \mathrm{D}$ printing as a substantial layer thickness is laid down with a single pass.

According to Skylar Tibbits, " $4 D$ printing where the 4th dimension entails a change in form or function after $3 D$ printing, is one recent example of programmable matter that at allows objects to be $3 D$ printed and then self-transform in shape and material property when exposed to a predetermined stimulus, such as being submerged in water or exposed to heat, pressure, current, ultraviolet light or other energy source"

From a design point of view, similar shrinkage was observed in the fabric substrates with different patterns during the MIPM and PAPM workflows discussed in the fourth chapter. This paved the way for two design directions to perform 3D/4D printing using bio-composite materials.

1.3D print patterns printed onto fabric substrates using the agar medium and allow them to dry until they achieve the desired shrinkage, thus transforming the substrates into predefined 3D forms.

2. 3D print patterns using mycelium inoculated printable agar medium, allow the incubation of mycelium and let the mycelium patterns dry to achieve shrinkage during desiccation. 
However, due to the following practical difficulties, the second design direction was not adopted by the author during the next stage of designing haute couture garment forms.

1. Constraints in sterilizing the robotic arm, printing table and other accessories for large-scale pattern extrusions.

2. No access to a highly controlled environment, which leads to easy contamination of the mycelium growth.

3. No access to a large-scale industrial oven to desiccate full-sized garment fabrics.

The notion of using natural shrinkage to generate 3D forms in garments became the focus of this research, as it was found that the shrinkage that transformed the 2D printed patterned on the fabrics into 3D/4D garment forms presented a unique design opportunity. At the beginning of this study, the 3D/4D aspect was the primary focus of this research and was discussed in detail in the literature review.

The next section discusses how this design direction was implemented using three digital workflow processes for the production of haute couture garment forms.

1. Bio-based 4D printing for haute couture fashion

2. Fashion garment production

3. Adoption of digital technologies in the production process

\section{SHRINKAGE}

Usually, shrinkage in fabrics is viewed as a negative impact on the finished garments. However, in the current study, the felting shrinkage of fabric is adopted as a design concept, where the fabrics are intentionally allowed to shrink, self-tailoring to body forms. 


\section{THE WORKFLOW OF BIO-BASED 4D PRINTING FOR HAUTE COUTURE FASHION}

The initial, yet the most crucial phase of any research-based design development process is the analysis of innovative opportunities. With all the global trends progressing towards sustainability, the author not only researched the developments in bio-composite material for 3D printing but also extended the study by developing a method to produce physical garment forms that incorporate a natural system to shape fabric to human body forms.

Here, the garment design process was inspired by two keys concepts: the natural shrinkage of fabrics with 3D printed printable agar medium patterns, and the production of garments using the concept of A-POC designs. 


\section{A-POC DESIGN CONCEPT}

Introduced to the world by Japanese Designers Issey Miyake and Dai Fujiwara, this unique concept uses the entire piece of fabric to produce a garment that significantly reduces fabric waste. Fabrics used to produce A-POC garments are created in continuous tubes, within which lie both shape and pattern, by a computer programmed industrial weaving machine. (A-POC (A Piece of Clothing) by Issey Miyake, 2008)

Inspired by the above concepts, the shrinkage design direction uses uncut flat fabric and natural shrinkage to produce 3D form. Presented next, is the detailed workflow of the design development approach of garment forms using 4D printed biocomposite materials obtained through experiments based on the Material Driven Design method, Figure 5.1 
FIGURE 5.1

Workflow for Fashion Design

Process, Innovation, and Practice

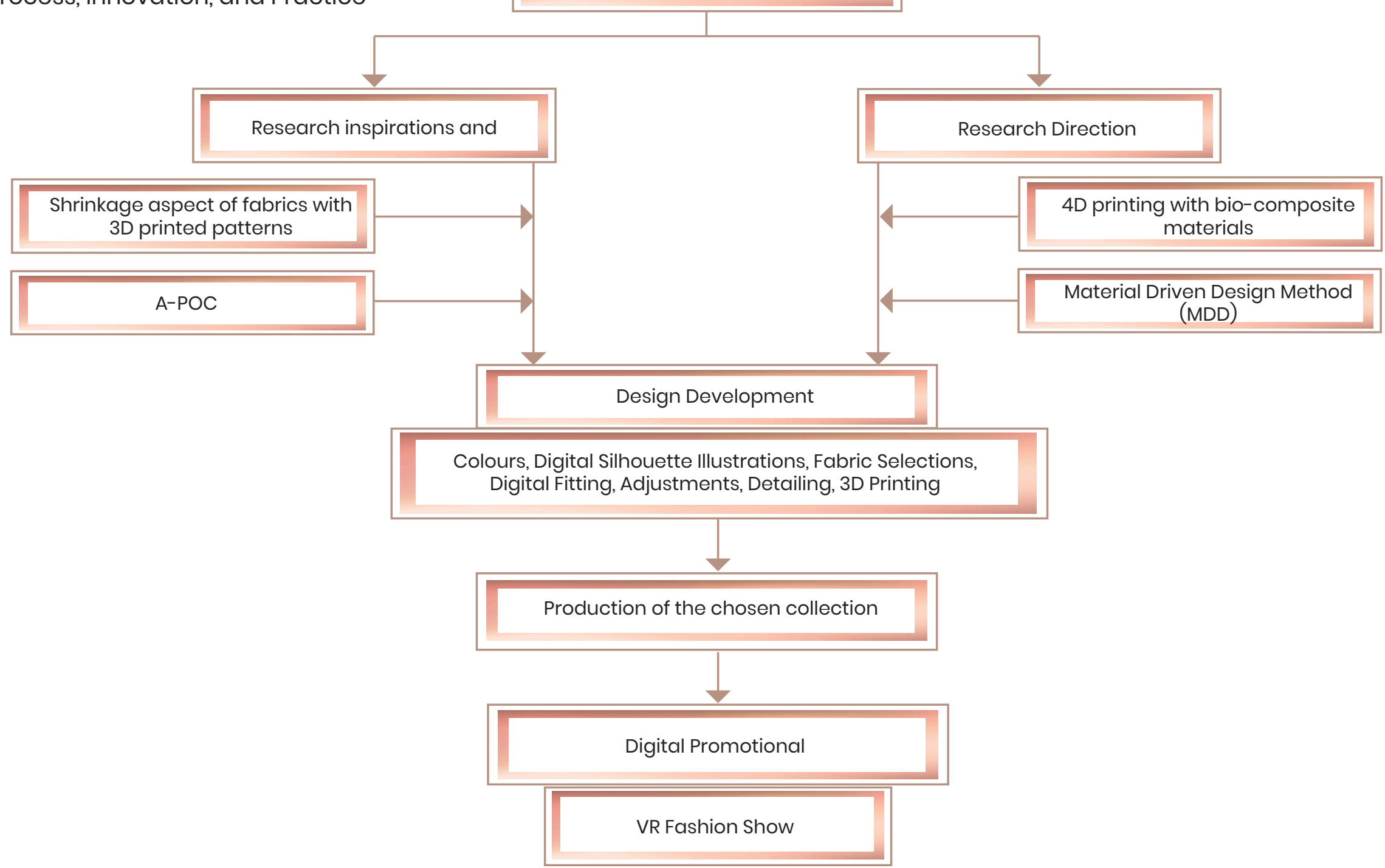

Source: Author's work (2021) 


\section{THE CONVENTIONAL WORKFLOW OF FASHION GARMENT PRODUCTION}

\section{THE DIGITAL WORKFLOW}

In the digital workflow developed by the author, sustainability is directly involved in the production of haute couture garments, through which three physical practices in the conventional design workflow could be eliminated as highlighted

in the figure 5.2 .

The processes of converting the ideas and themes into hand sketches during the pre-production step, 2D pattern making, 3D toil making, physical fitting and adjustments in the production step.

Initiation of the process was very similar to the conventional process, where everything begins with a sketch or an idea. This idea was then transformed into a digital silhouette illustration using the software, Adobe lllustrator.

The next step was to create a 3D rendering using the developed silhouette illustration. Here, there were two software options, either to use Rhino7 from Rhinoceros $3 D \otimes$ or the software CLO 3D. 
FIGURE 5.2

The Conventional Workflow of Producing Haute Couture Fashion Garments

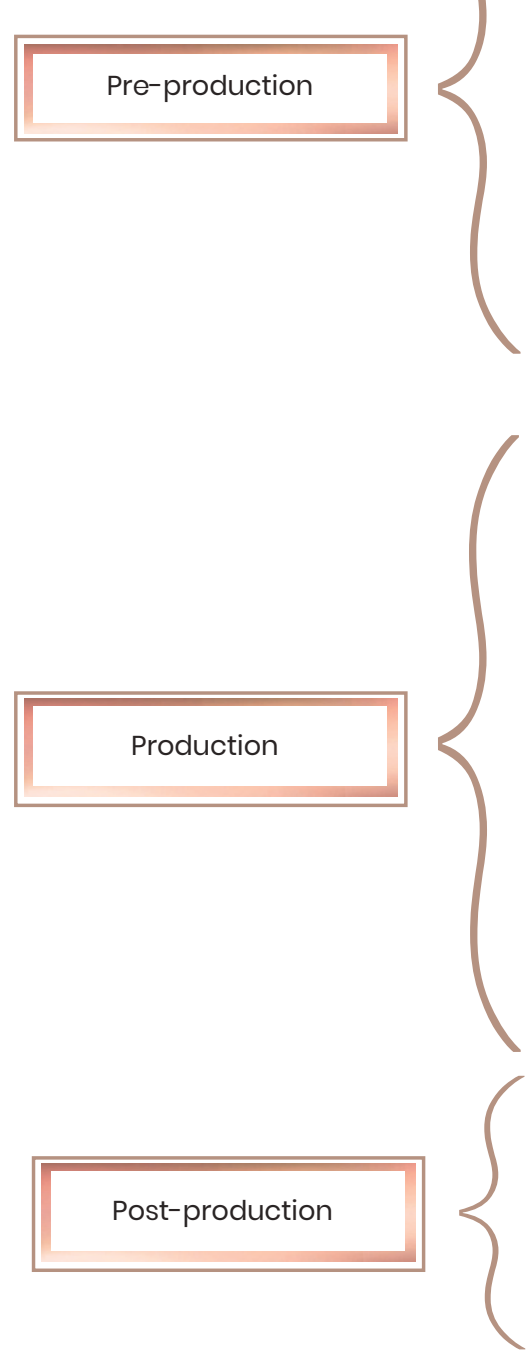

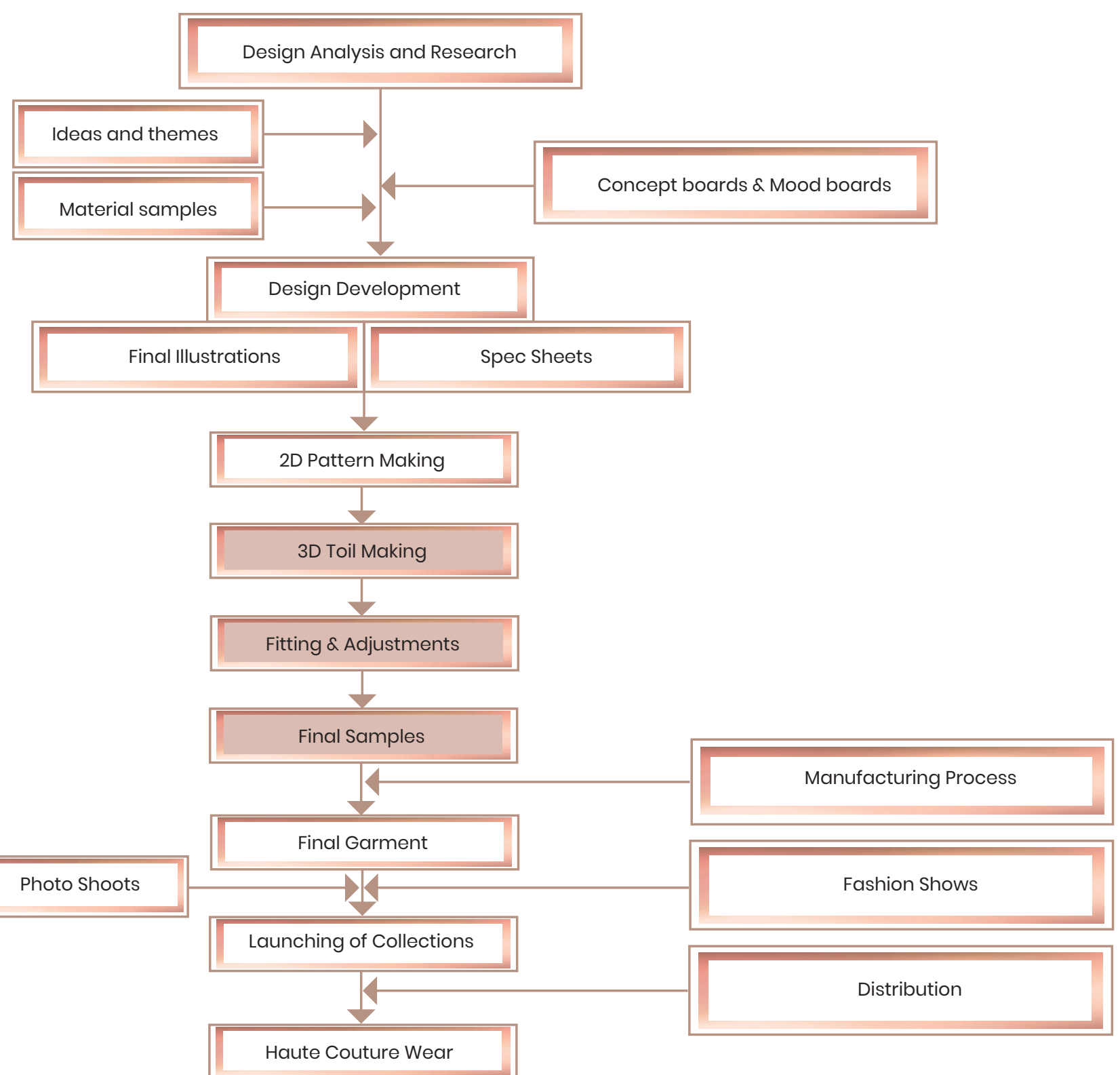

Source: Author's work (2021) 


\section{PROS AND CONS OF USING RHINO7 AND CLO3D}

The advantage of using Rhino7 was that it is capable of directly generating Grasshopper scripts (discussed in detail in Chapter 4), which are then plugged into the robotic arm 3D printer. However, there are practical limitations in using Rhino7 for 3D rendering of fashion garment designs for the following reasons.

- Rhino7 plug-in Grasshopper, has been primarily developed for Architectural Designs.

- It has accessibility constraints in working with human body shapes and shrinkage of fabrics.

CLO3D, on the other hand, is a computer software developed for the exclusive use of digital costume design, customised costumes, and character designing. Some of the key features of CLO3D are its real-time interactivity, unlimited designs and styling, accurate materials, and streamlined processes. (CLO I 3D fashion Design Software, n.d.). Therefore, the generating and customising clothing models are much easier using CLO3D than using Rhino7.

The only disadvantage of CLO3D was its inability to generate Grasshopper scripts for the 3D rendered designs.

These limitations were overcome by combining the use of both software packages: CLO3D for designing and customising the virtual model and then exporting the outcomes to Rhino7 and Grasshopper scripts to generate instructions for the robotic arm. 


\section{D FASHION RENDERING USING CLO3D}

The first step is to create a customised avatar. CLO3D offers a range of in-built avatars for designers to choose from, with pre-defined body sizes. If further customisation is required to match specific measurements, the Nettelo 3D body scanning app can be used to perform a body scan of a live model and generate a virtual clone avatar that can be exported to CLO3D.

Once the avatar is created, the selection of fabrics is the next step from the in-built libraries of CLO3D. Advanced features are available, where designers can opt for additional software like Adobe Substance Designer to define customised fabric forms and export them to CLO3D.

Once these steps are completed, the parametric 2D pattern with internal polygonal lines is generated on the 2D Window of the CLO3D software. The practical shrinkage data obtained for each of the core design pattern experiments in Chapter 4 were then simulated on the virtual fabric by changing the relevant parameters, thus generating the exact body forms in the developed garment design model in the 3D Window.

Once the shrinkage data is fed in the computational outputs are produced and observed, the final CLO3D rendering is performed. Manipulation of the printed line length, orientation and configuration of the patterns can be adjusted to create the desired design output.

From this step onwards, Rhino7 software is used to transform the generated design 2D patterns to the physical extrusion process through the robotic arm extruder.

In exporting the generated final render from CLO3D to Rhino7, the 2D pattern and 3D model are saved in different file formats.

The 3D model is saved in .zpjr file format and the 2D pattern is saved in .pdf file format. 


\section{RHINO7, GRASSHOPPER SCRIPTING AND 3D PRINTING}

The saved 2D pattern is opened in Rhino7 through the graphical algorithm editor, Grasshopper, and converted into a Grasshopper script.

In the next step, the printing workflow is arranged using Grasshopper, which designates the operation path to the robotic arm printer.

Finally, this modified Grasshopper script is plugged into the robotic arm printer to perform the $3 \mathrm{D}$ printing using the agar medium on the fabric substrate.

Figure 5.3 illustrates the detailed digital workflow adopted in this thesis. 
FIGURE 5.3

The Digital Design Workflow

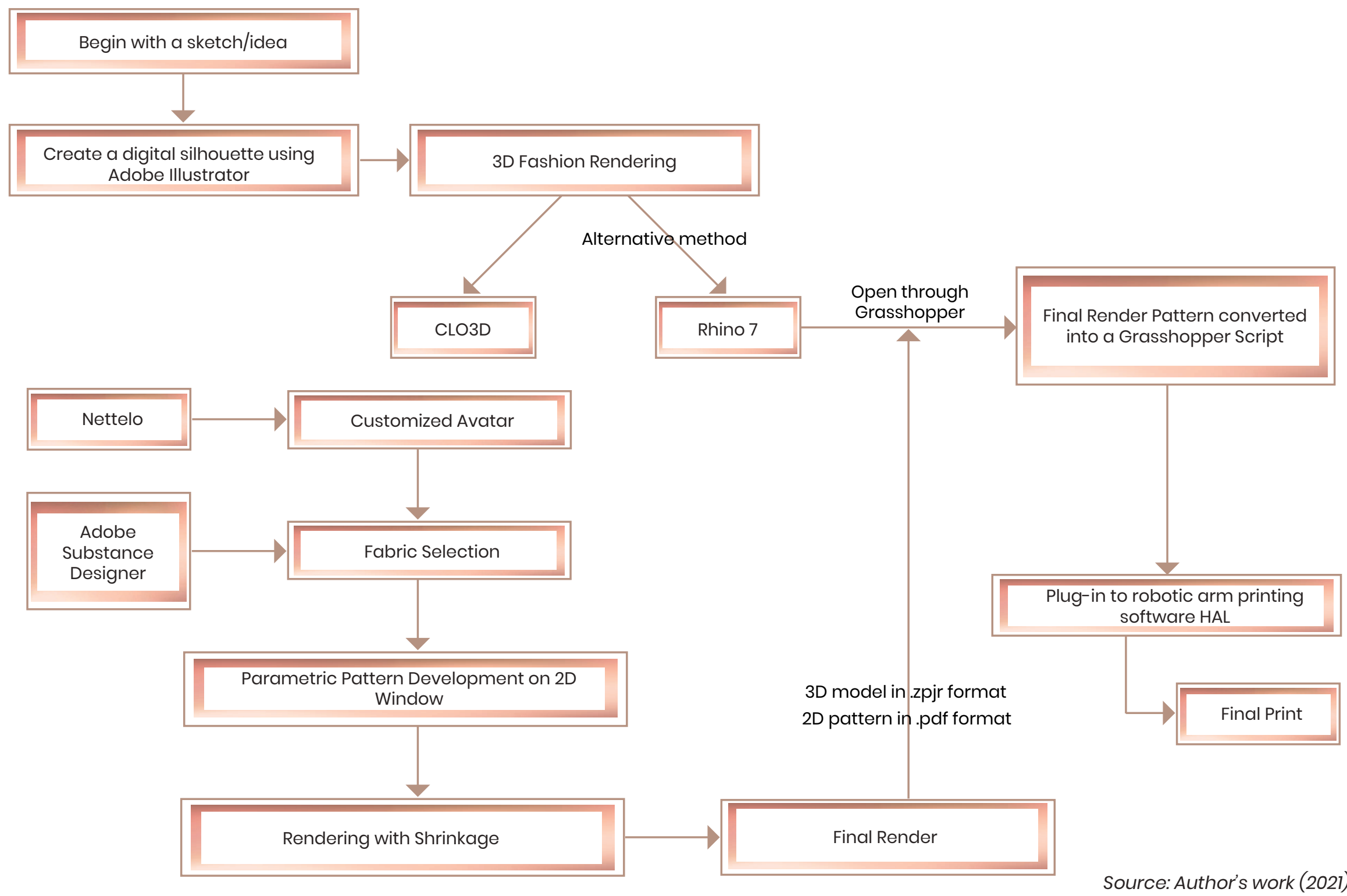




\section{FINAL DESIGNS}

Following the above digital workflow, an haute couture fashion collection was designed and developed to express a range of design opportunities.

\section{DIGITALIZATION OF THE DESIGN CONCEPT}

The design concept was originated from the line textures and colors of mushroom spawn prints and was inspired by a classic black dress and traditional hand smock techniques of fabric manipulation to enhance female body forms.

The mood board shown in figure 5.4 illustrates the origins and inspirations of the fashion collection "The Black Liner"

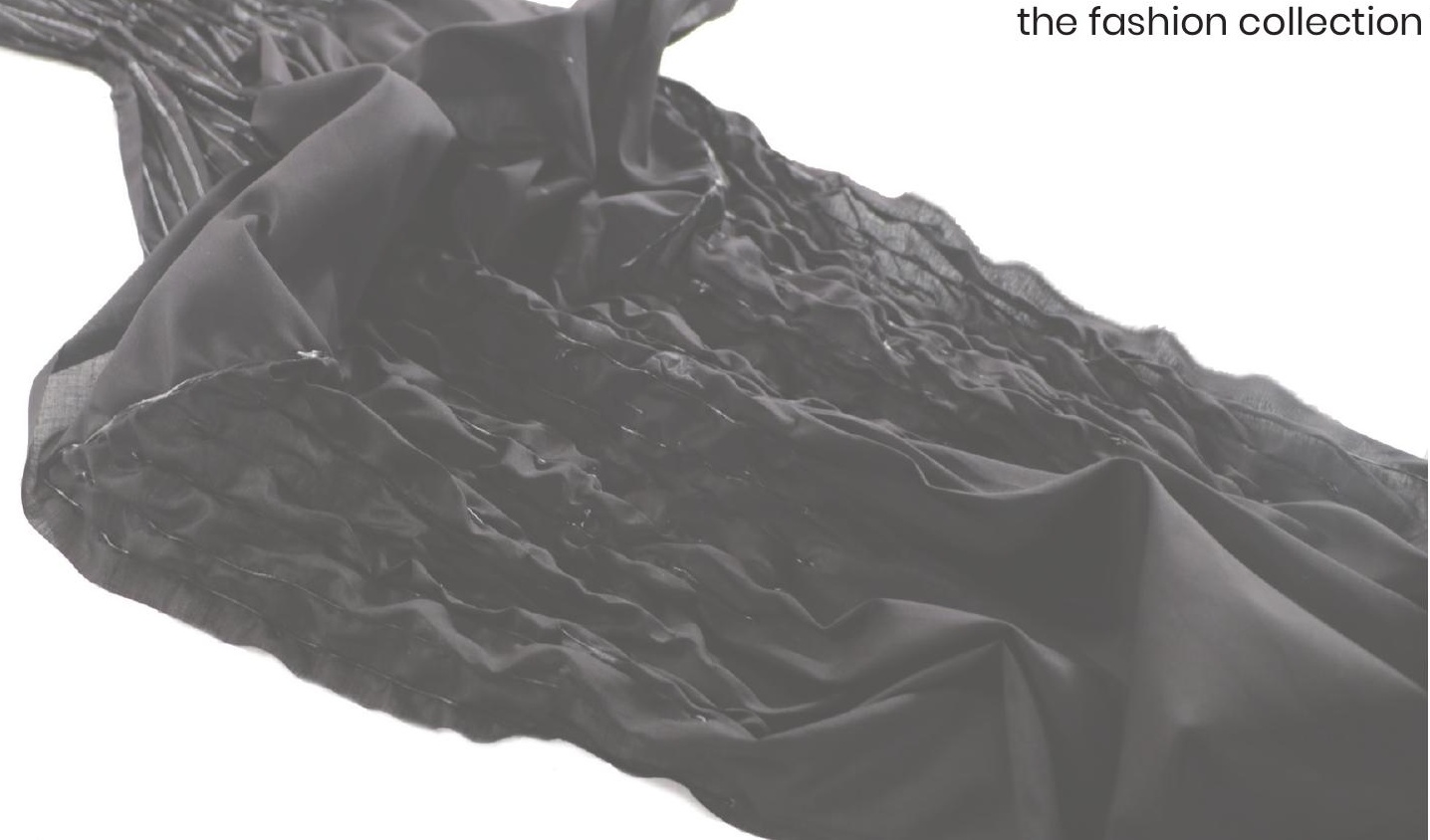




\section{FIGURE 5.4}

The Mood Board for the Development of the Fashion Collection

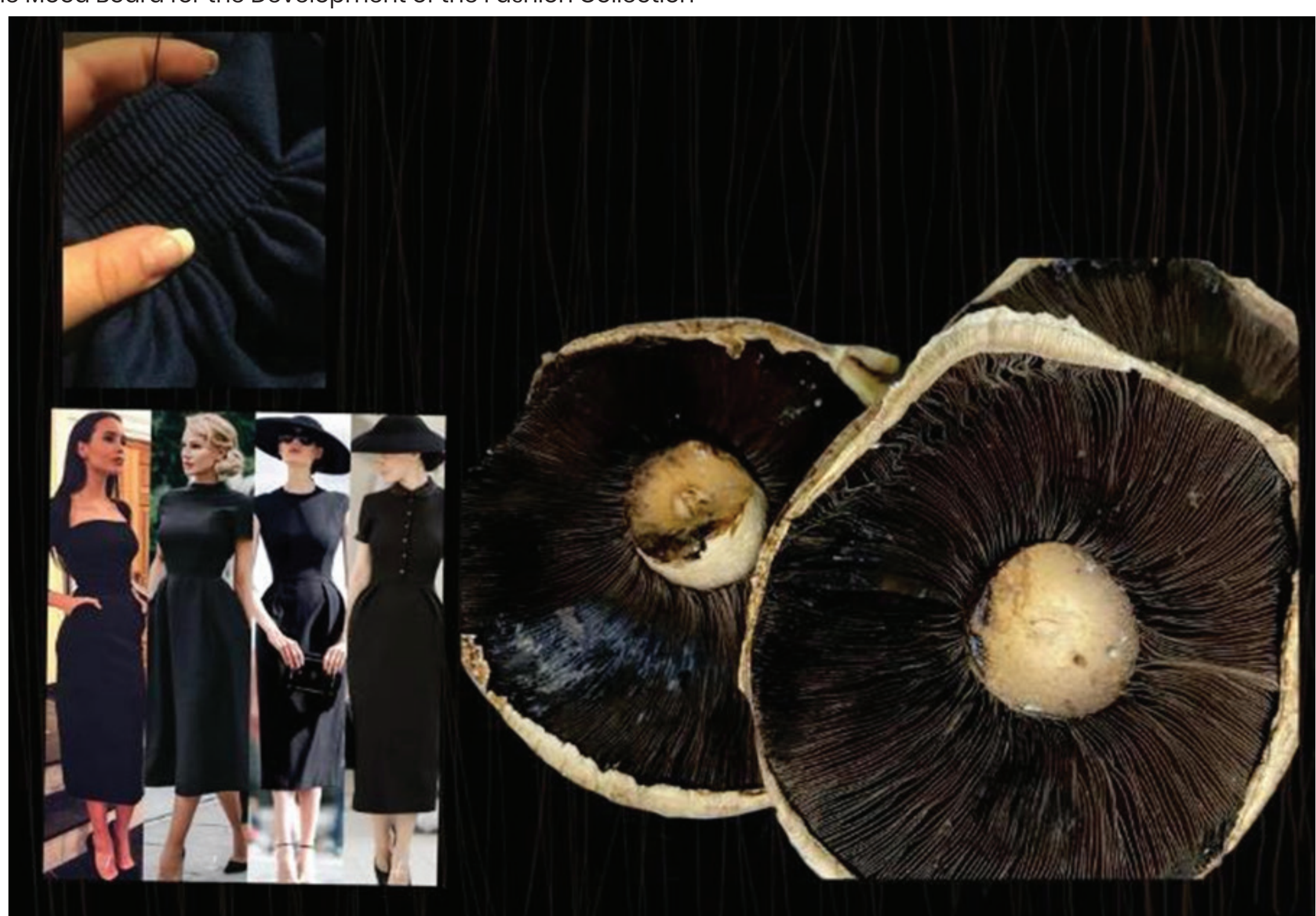

Source: Author's work (2021) 
In the mind of the A-POC concept, the following digital silhouettes of garment forms were created as demonstrated using PAPM with charcoal on $100 \%$ cotton fabric, as in figure 5.5 .

\section{FIGURE 5.5}

Digital Silhouettes Created for the Fashion Collection
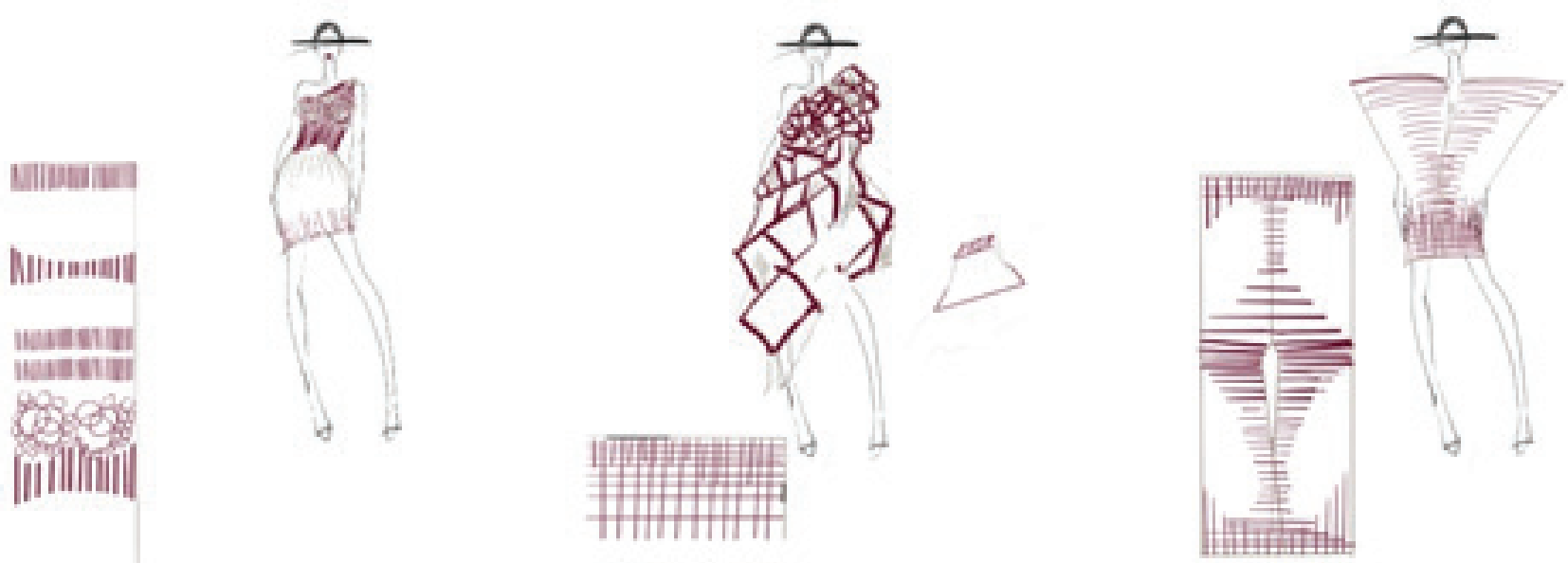

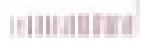
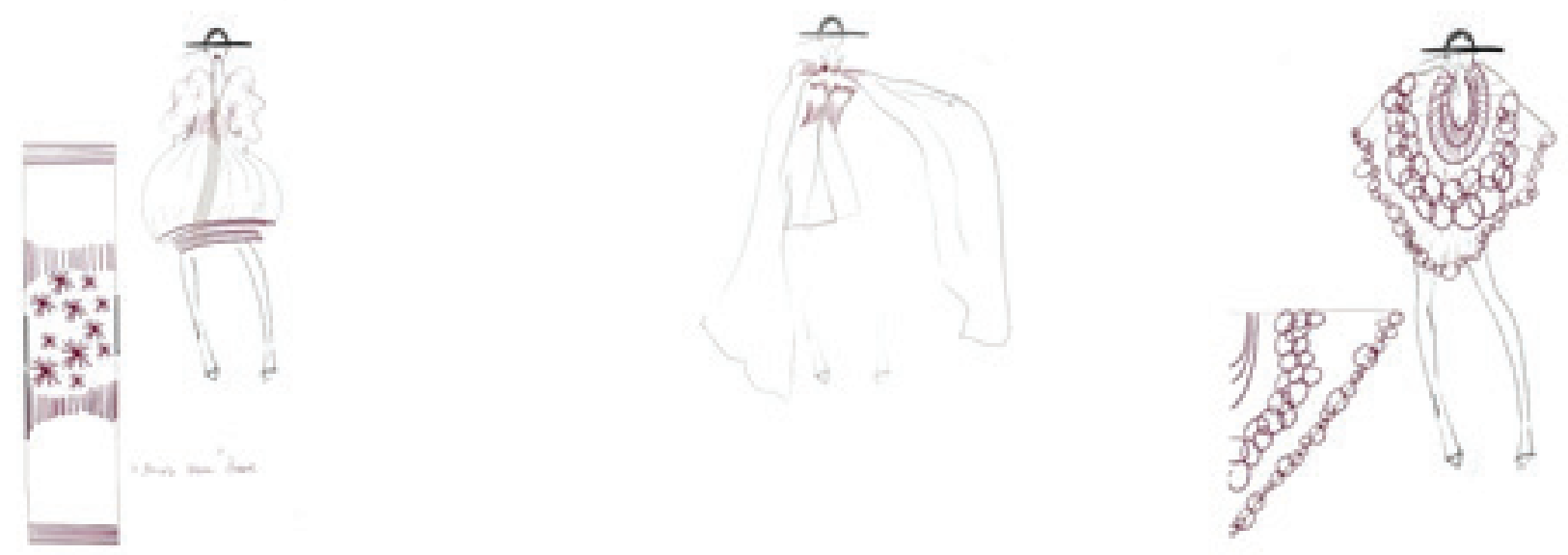

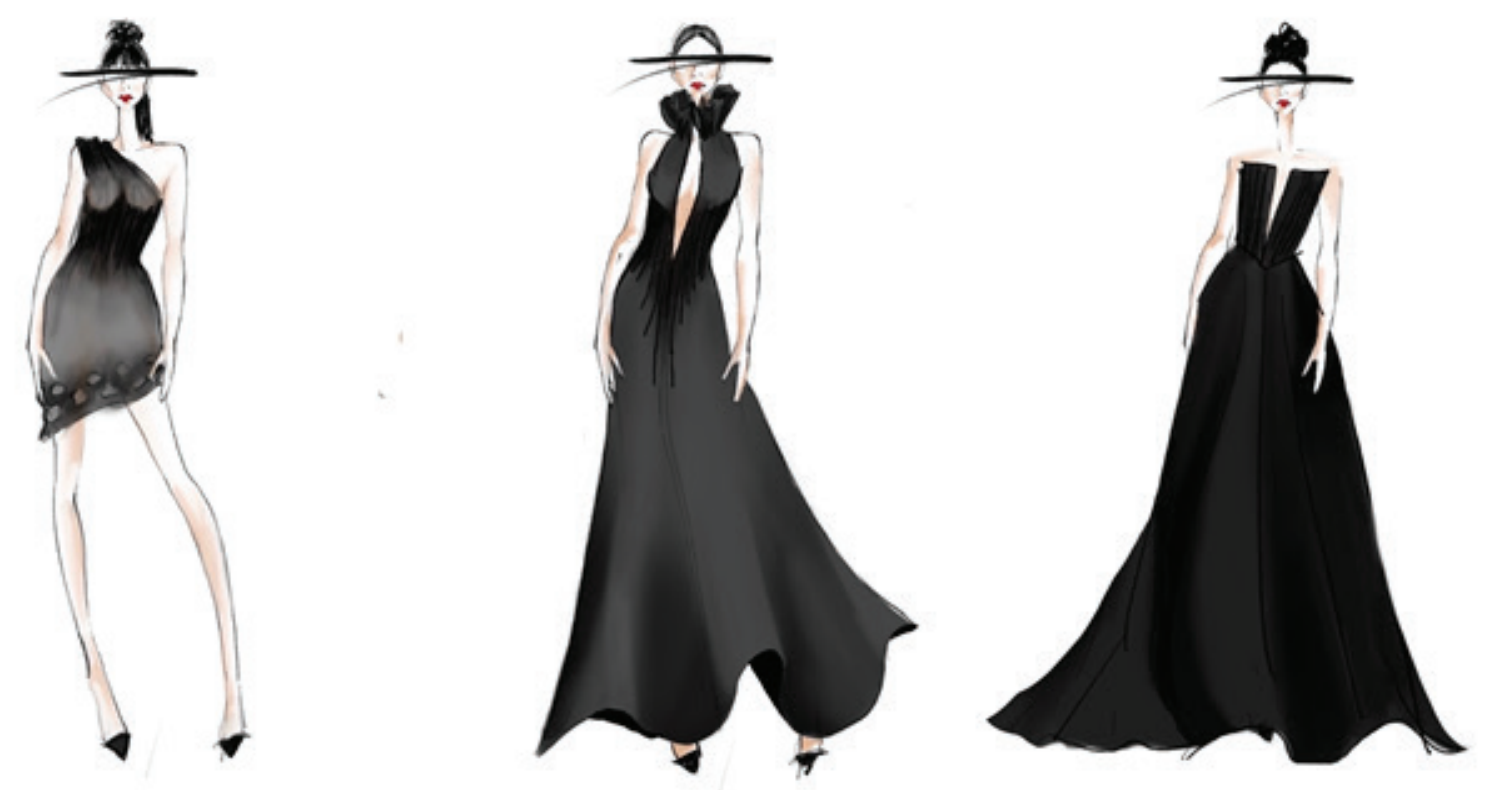

Using the shrinkage percentage analysis data from chapter 4 (figure 4.1), line patterns were placed onto the selected 2D fabric surface digitally and the rendering tools of CLO3D software were used to simulate the shrinkage values, thus, creating 3D form. 
THE LITTLE BLACK DRESS

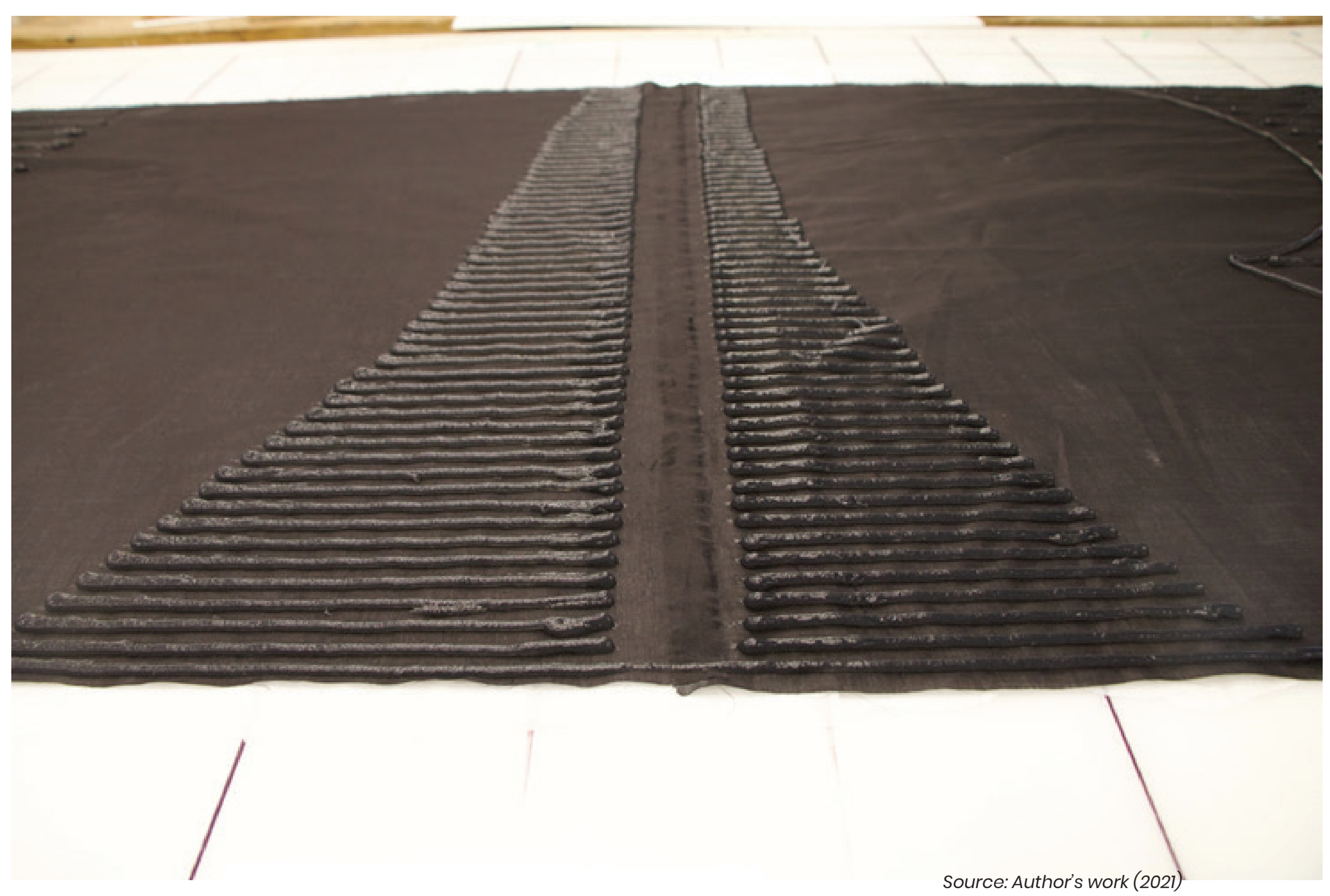


Type:

Fabric Size:

Design Characteristics:

2D $\& 3 D$ Windows of the CLO3D Software

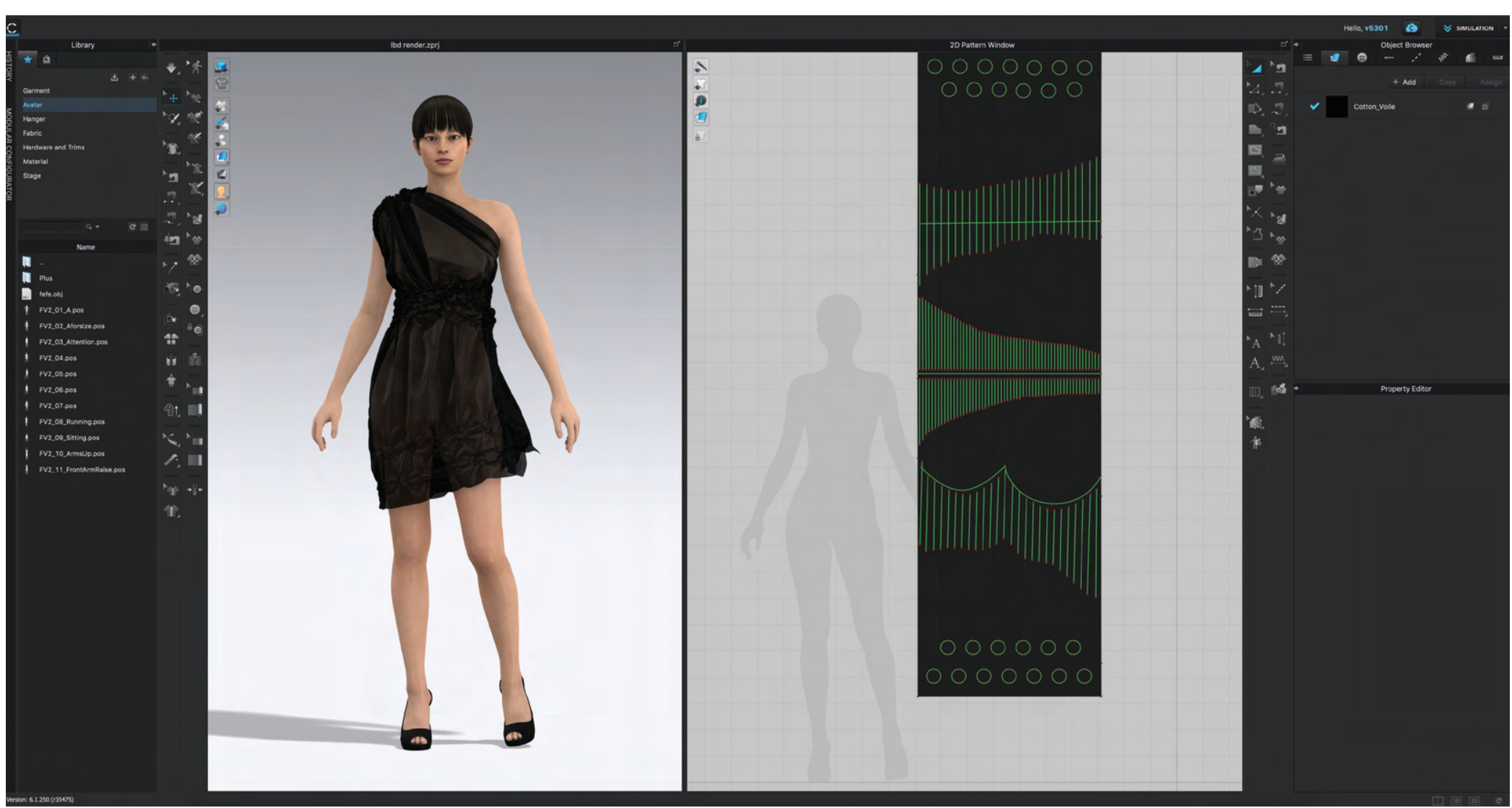

Source: Author's work (2021)

A dress over one shoulder

Single piece of $1500 \mathrm{~mm} \times 700 \mathrm{~mm}$

Pattern lines at the shoulder are densely placed to allow the width of $700 \mathrm{~mm}$ to shrink to $150 \mathrm{~mm}$. Pattern lines at the waist have been extruded to shrink and enhance the body form after shrinkage takes place.

FIGURE 5.6A 

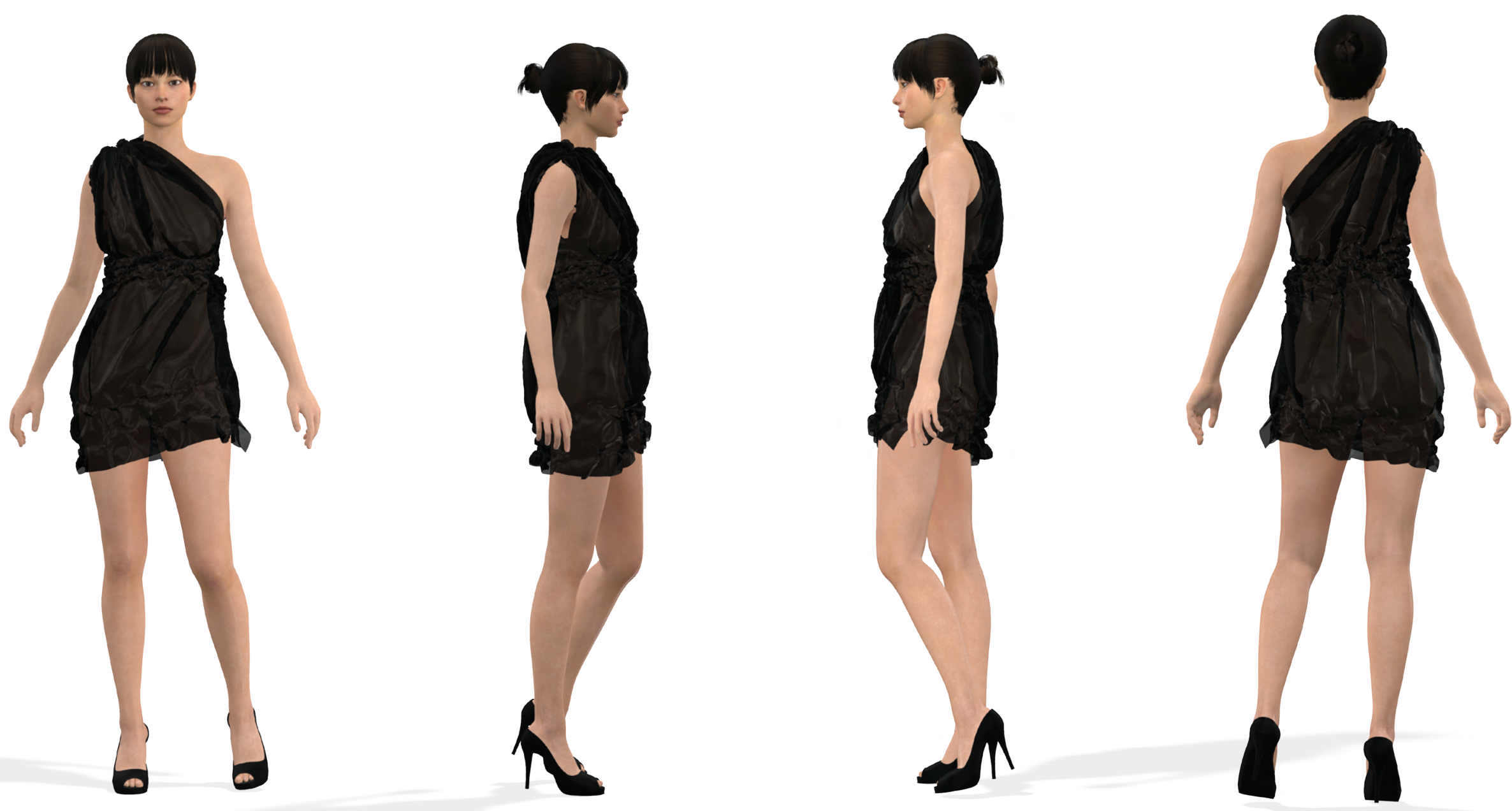
FIGURE 5.6C

Exported from CLO 3D -2D pattern into Rhino

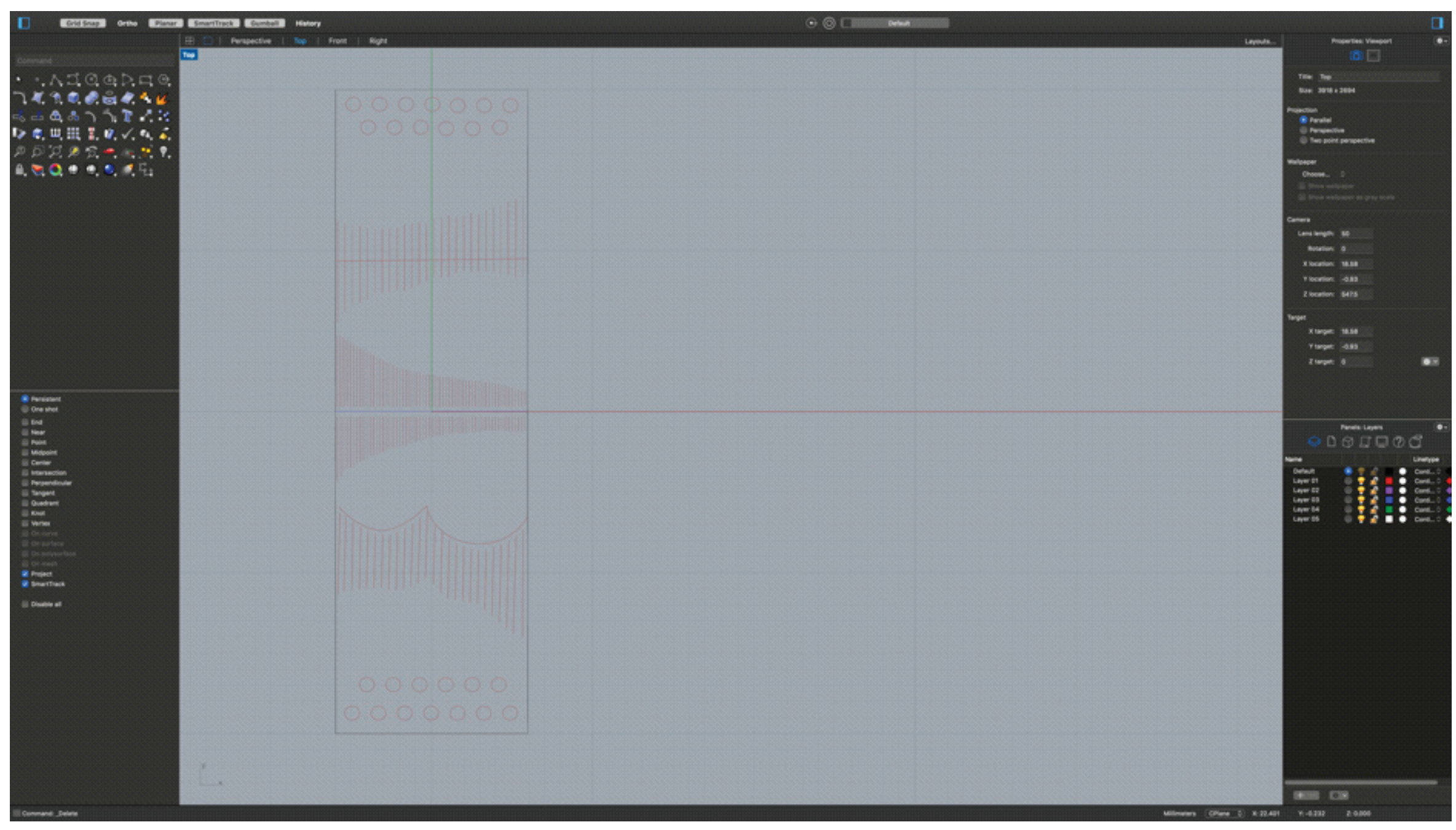

Source: Author's work (2021) 
FIGURE 5.6D

Grasshopper script

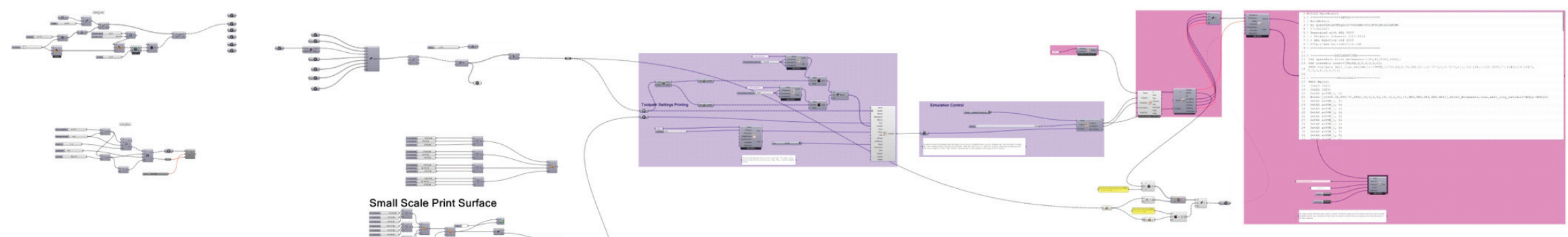

Tool path with robotic arm 3D printer

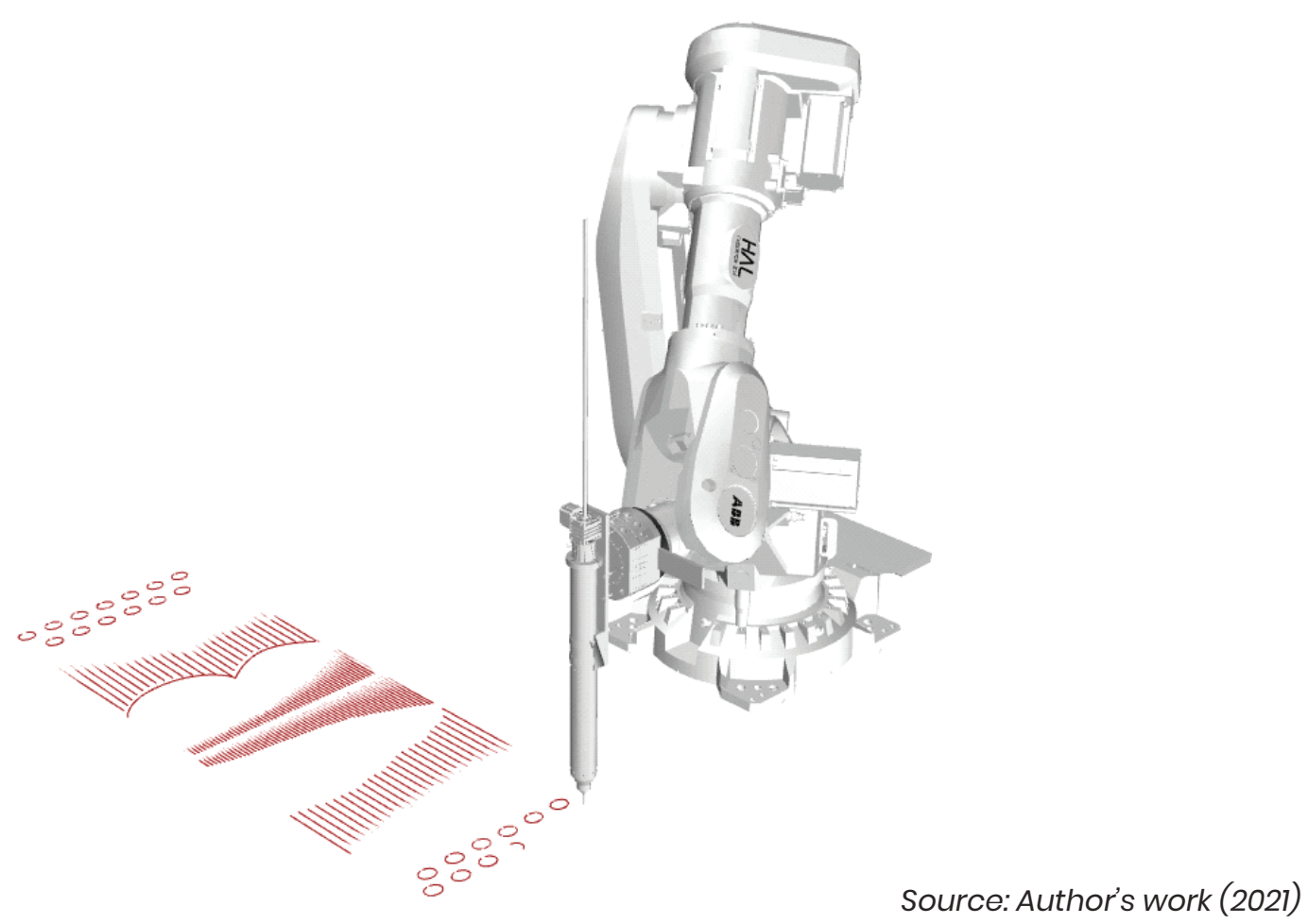


FIGURE 5.6E

Final print and process of drying

Before

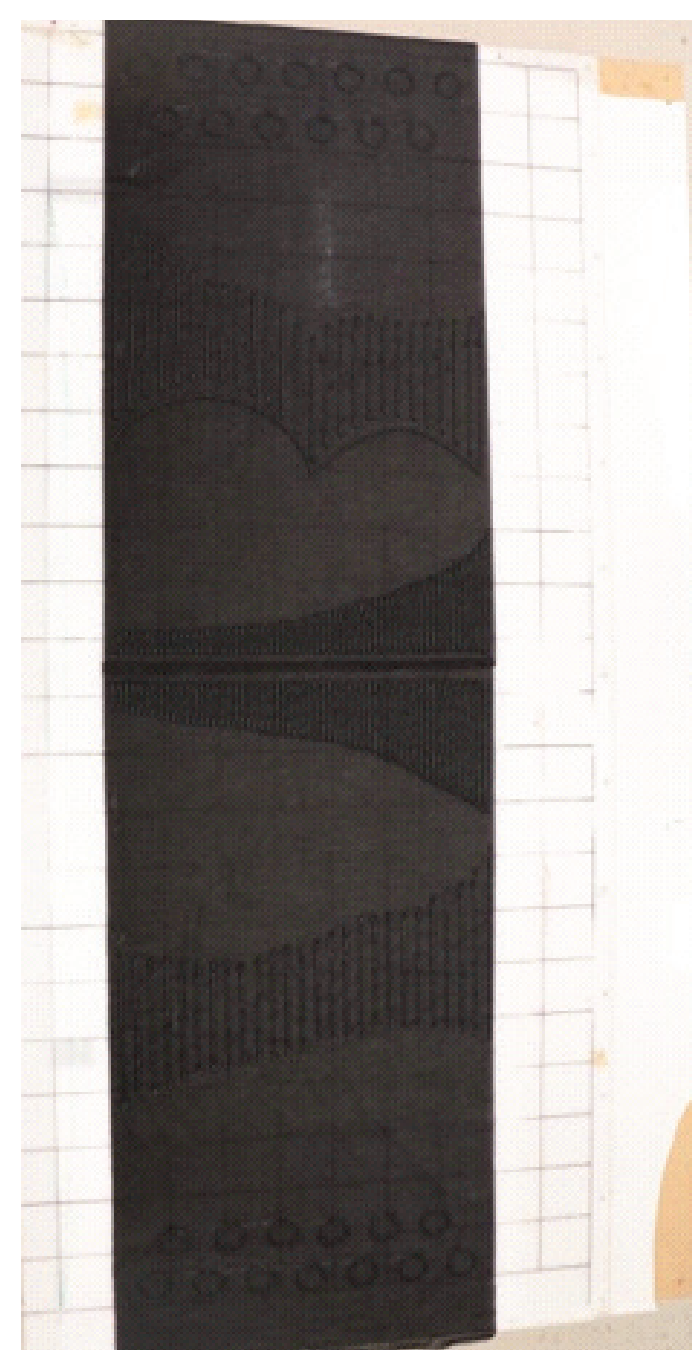

After 48 hours

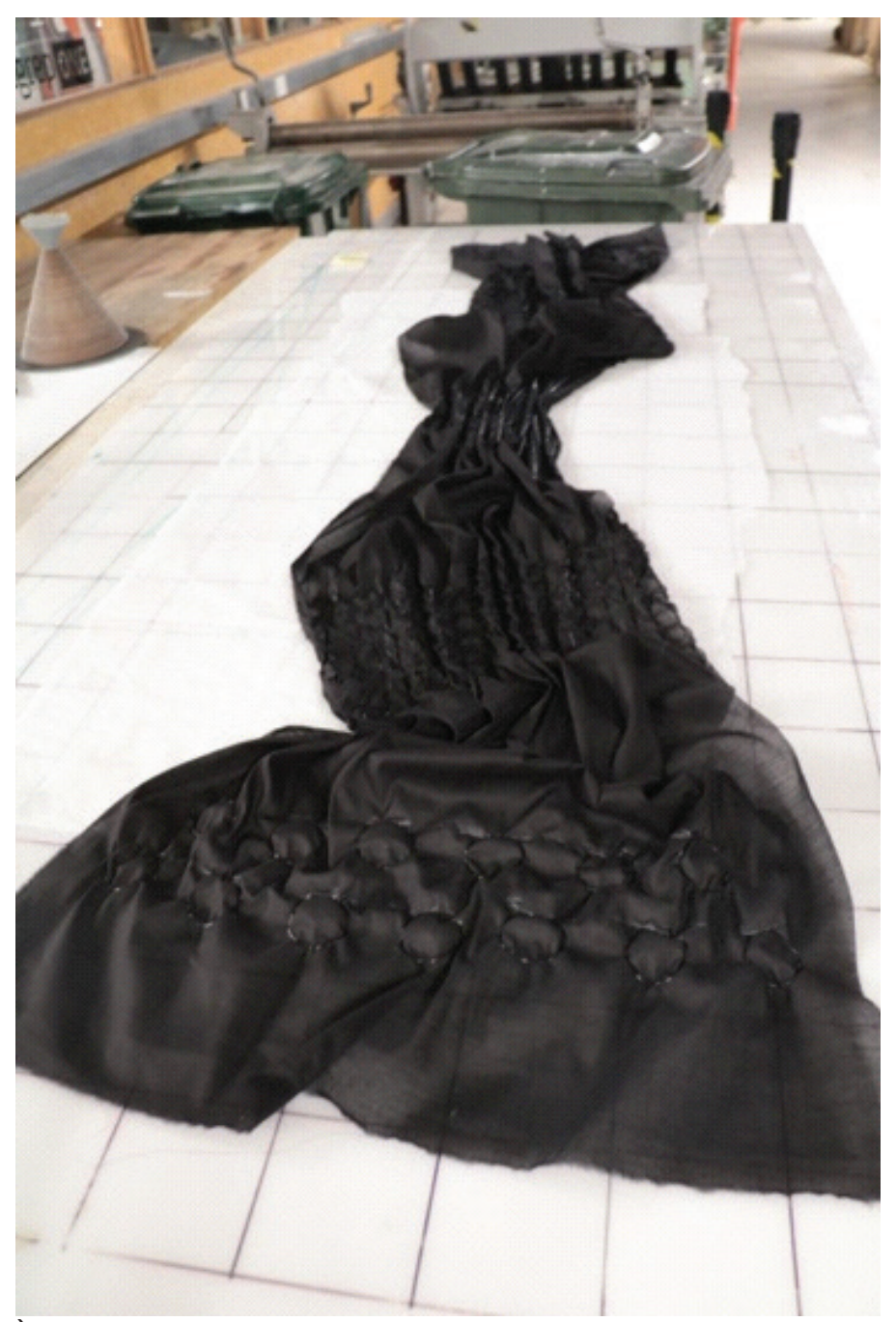




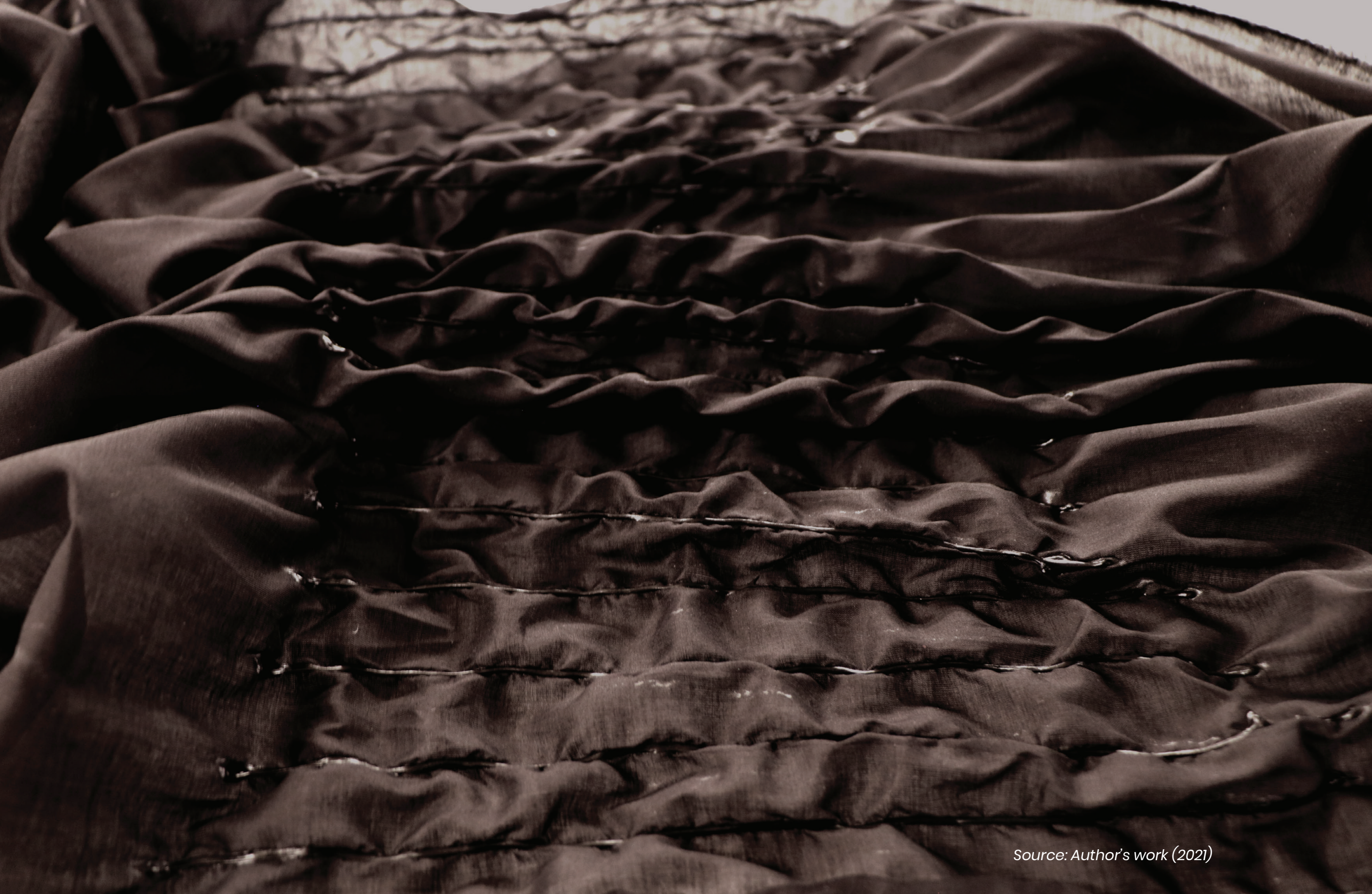


Final designs
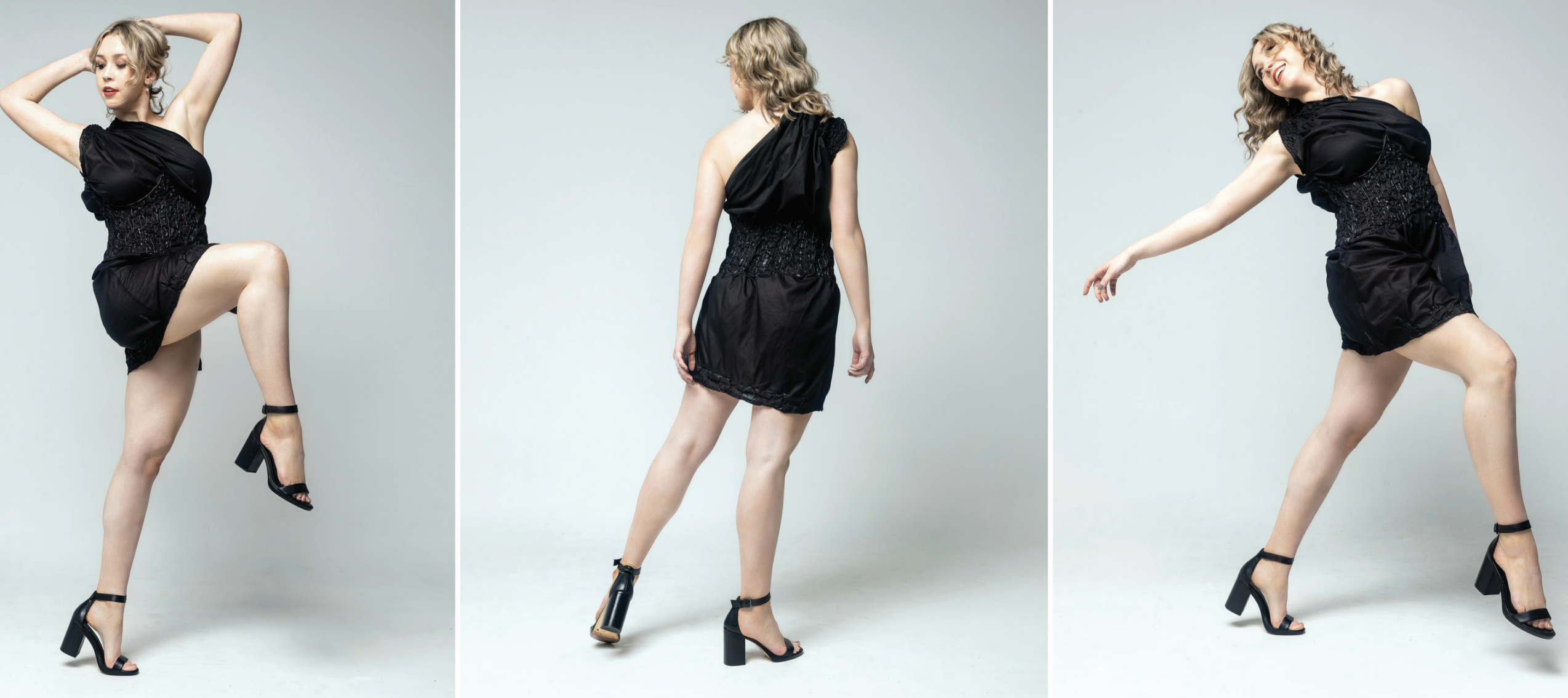


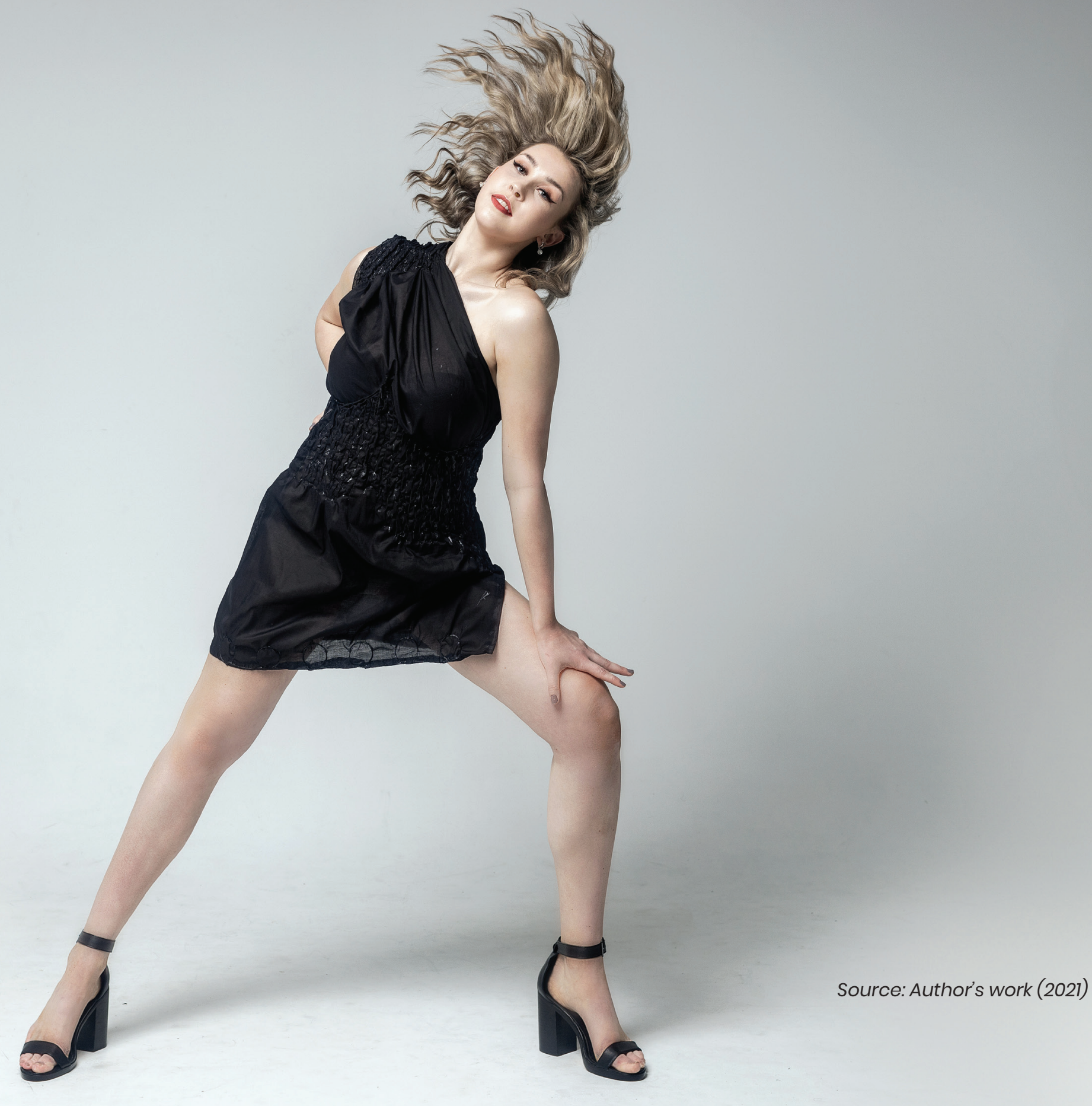




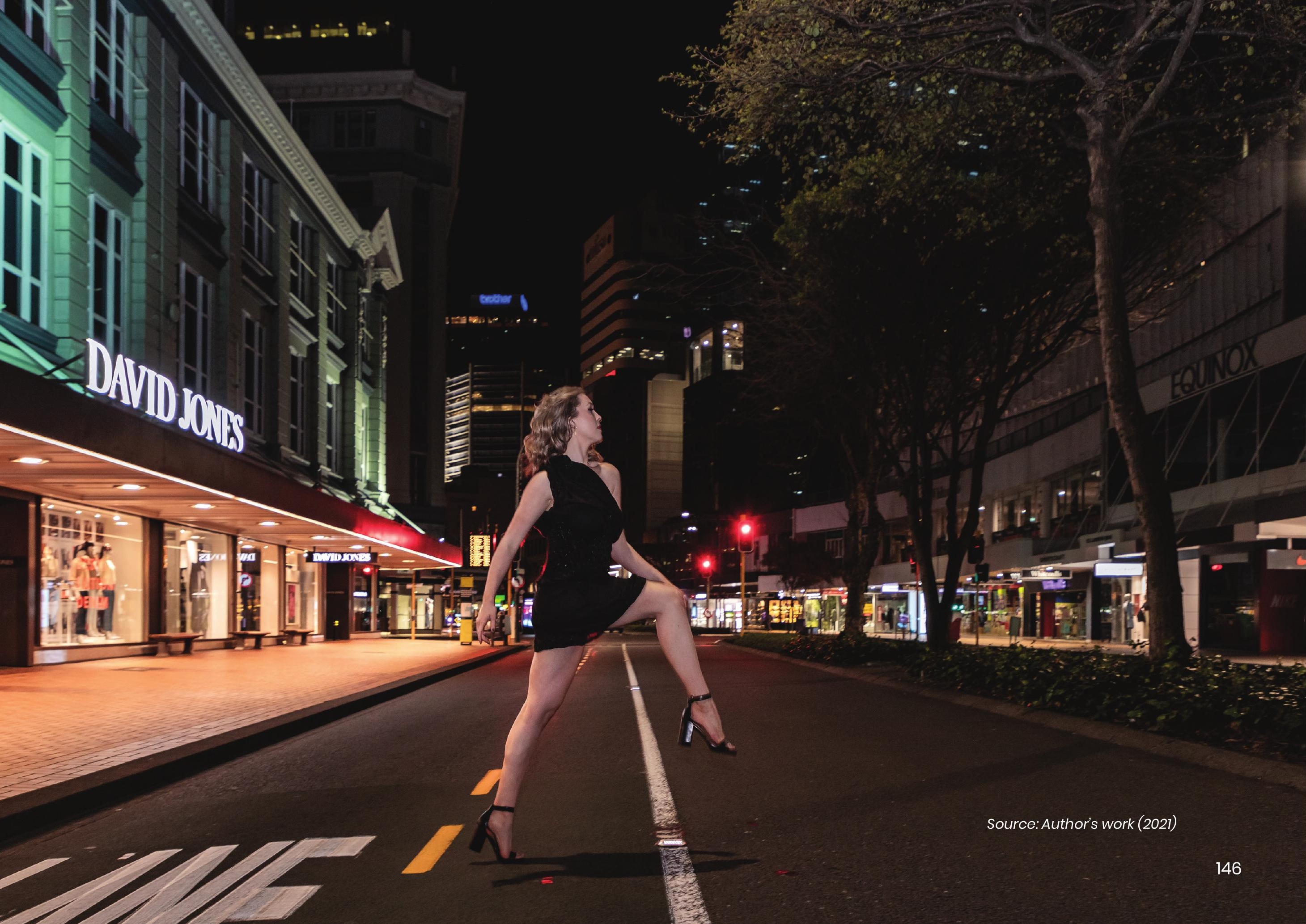


Type:

Fabric Size:

Design Characteristics:

\section{FIGURE 5.7A}

2D $\& 3 D$ Windows of the CLO3D Software

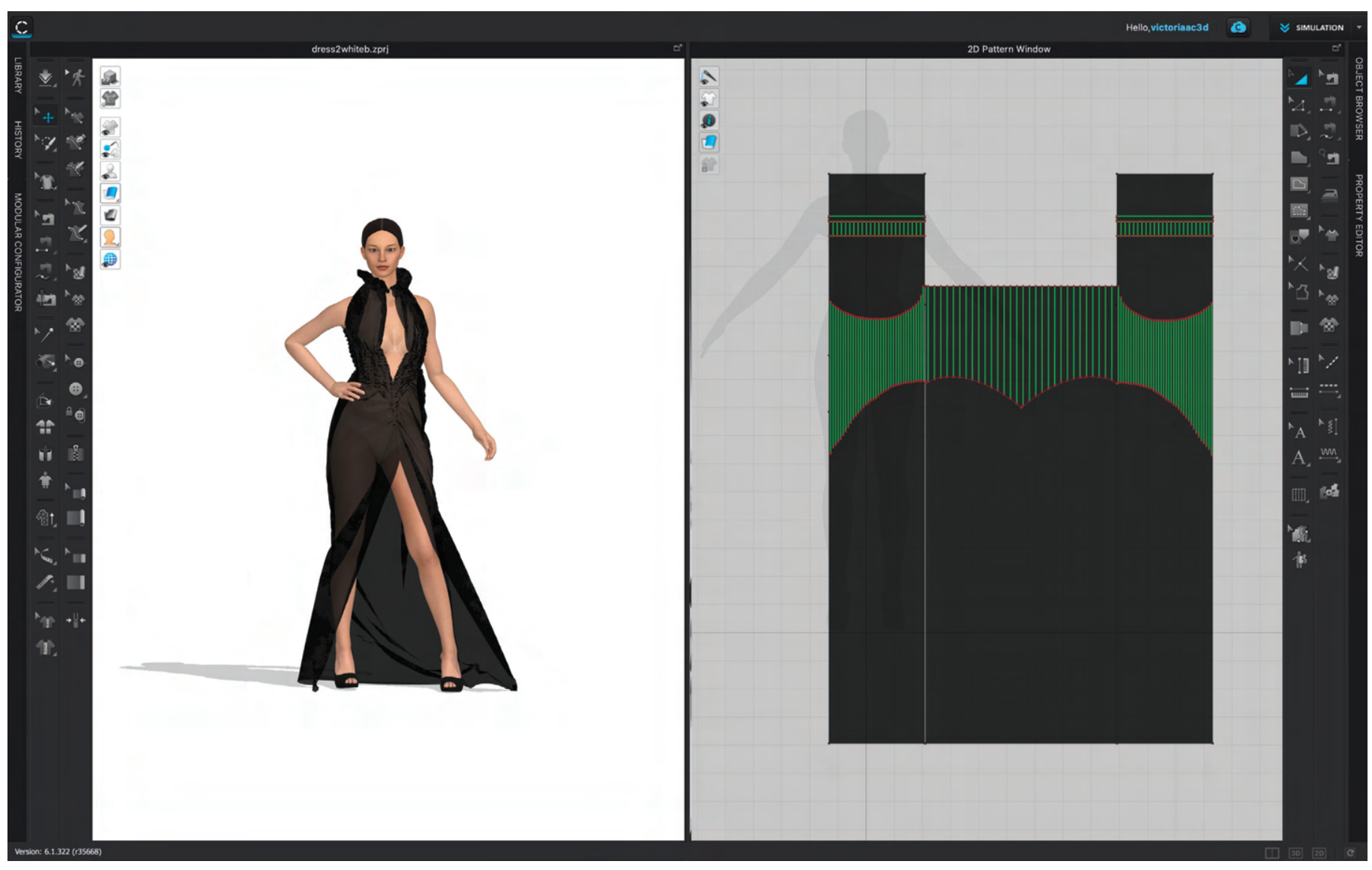

Source: Author's work (2021)
Single piece of $3000 \mathrm{~mm} \times 1400 \mathrm{~mm}$

Patterns placed on back, right and left sides.

On the right and left sides, neck pattern lines were placed to suit the neck form upon drying. 


$$
\text { Nit }
$$


FIGURE 5.7C

Exported from CLO 3D -2D pattern into Rhino

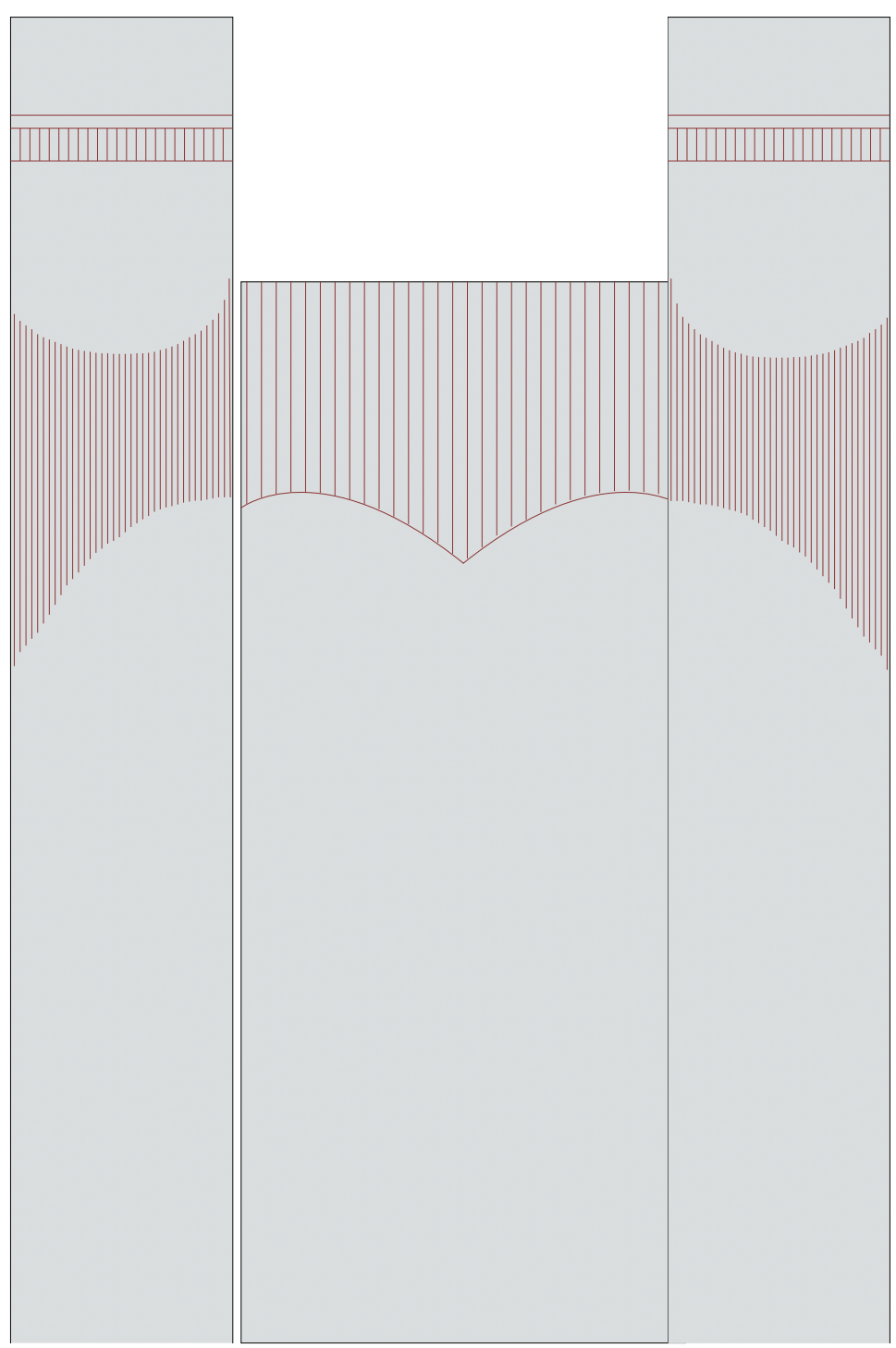


FIGURE 5.7D

Grasshopper script

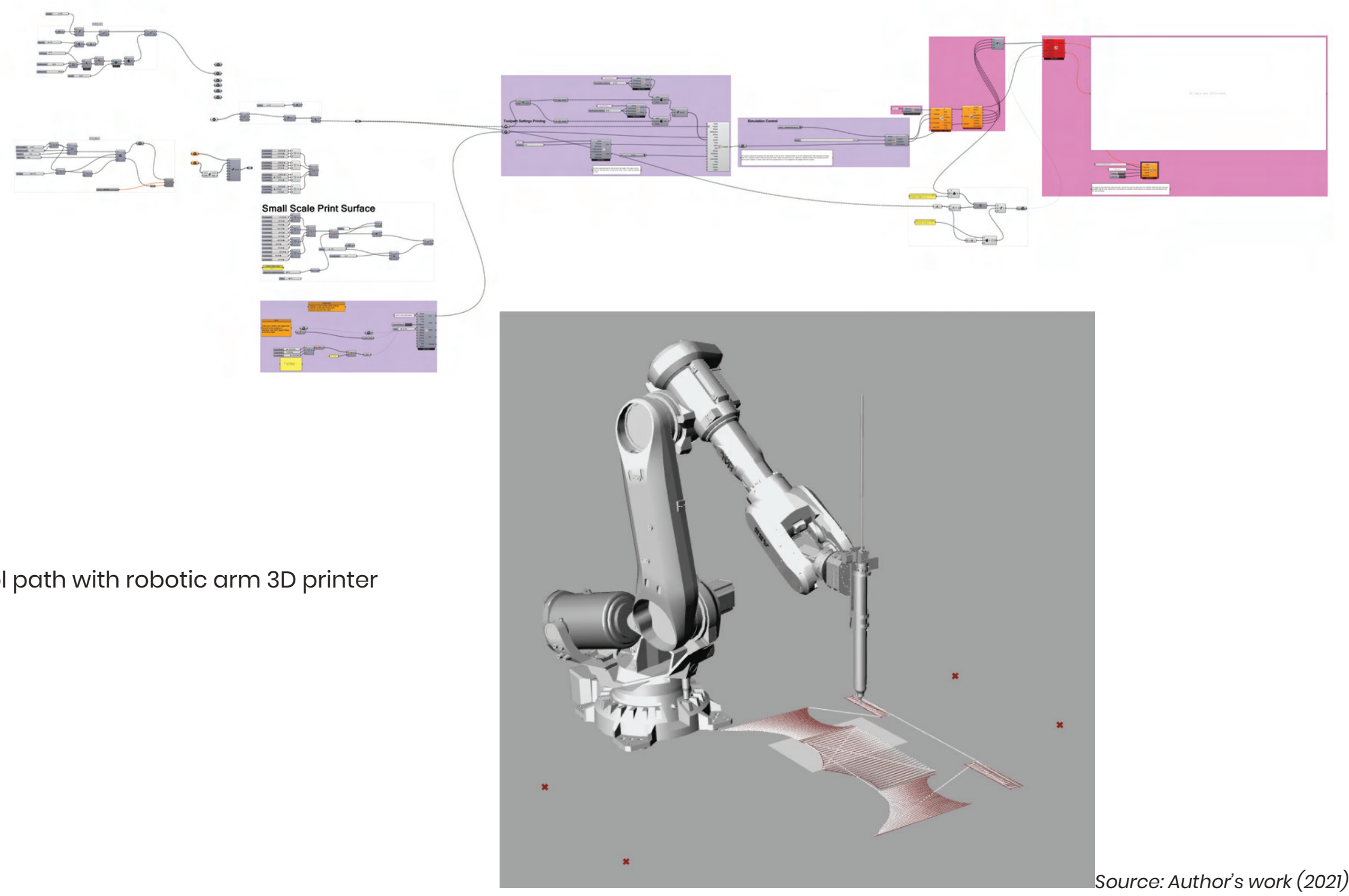



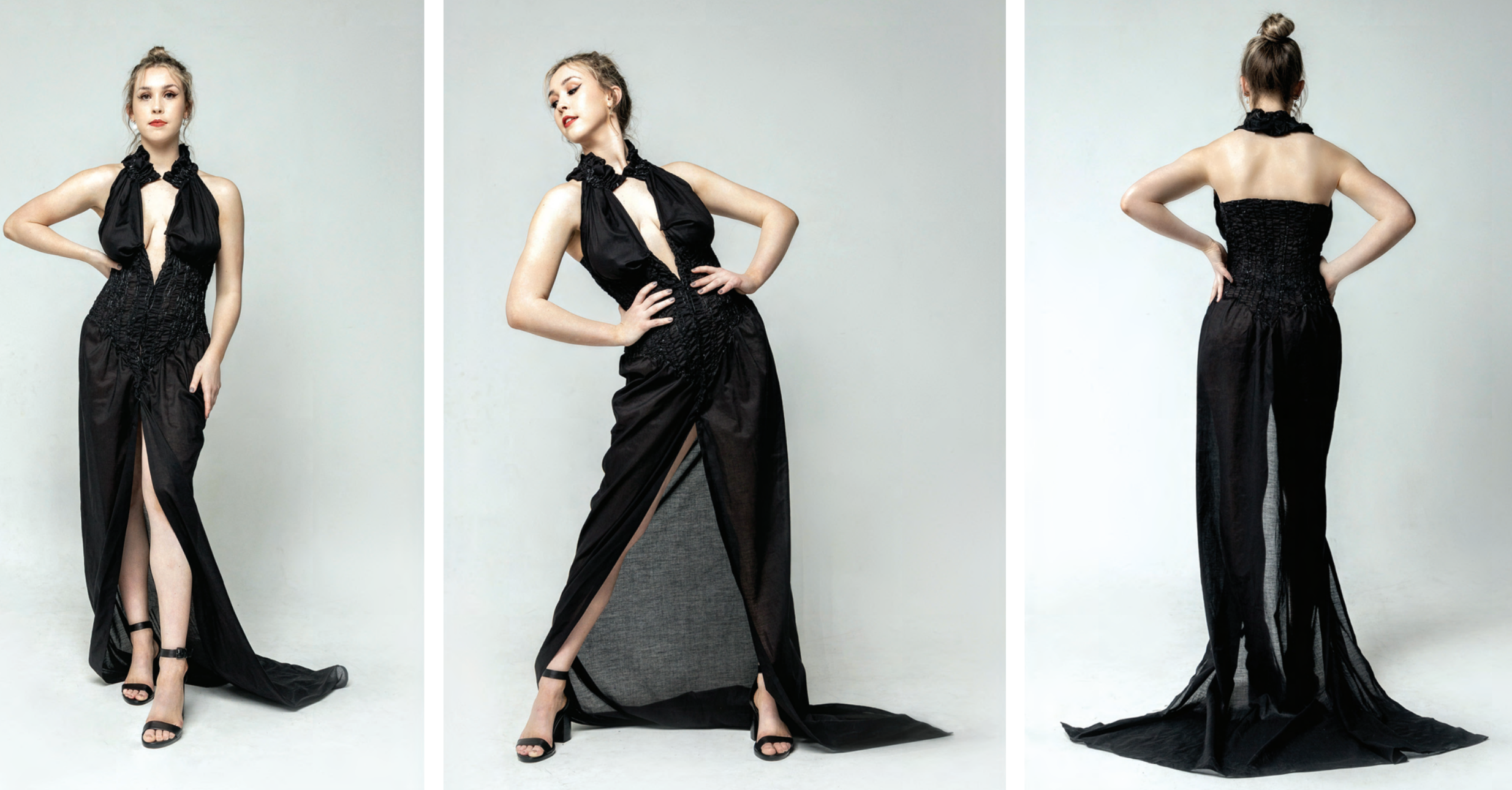

Source: Author's work (2021) 


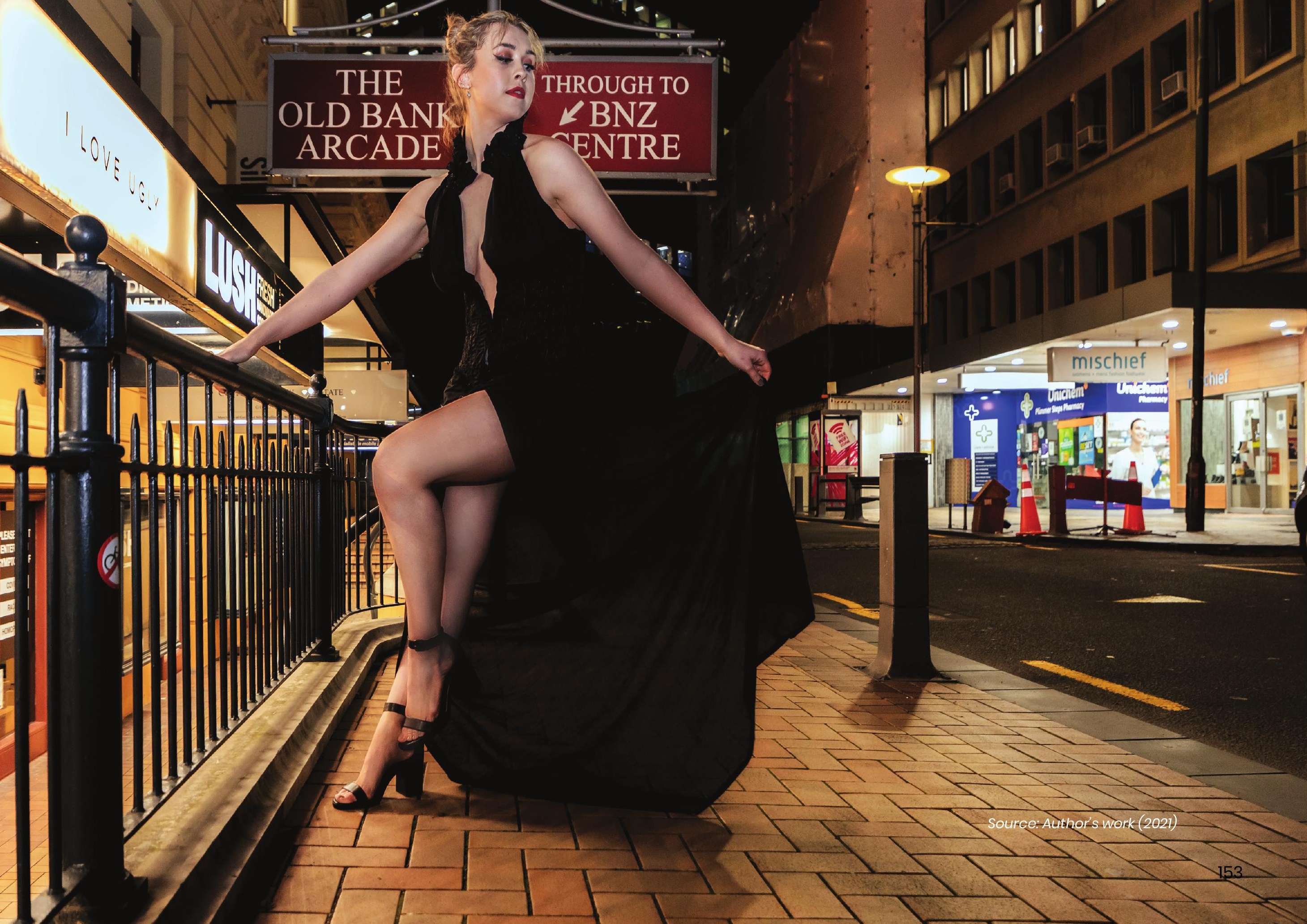


0

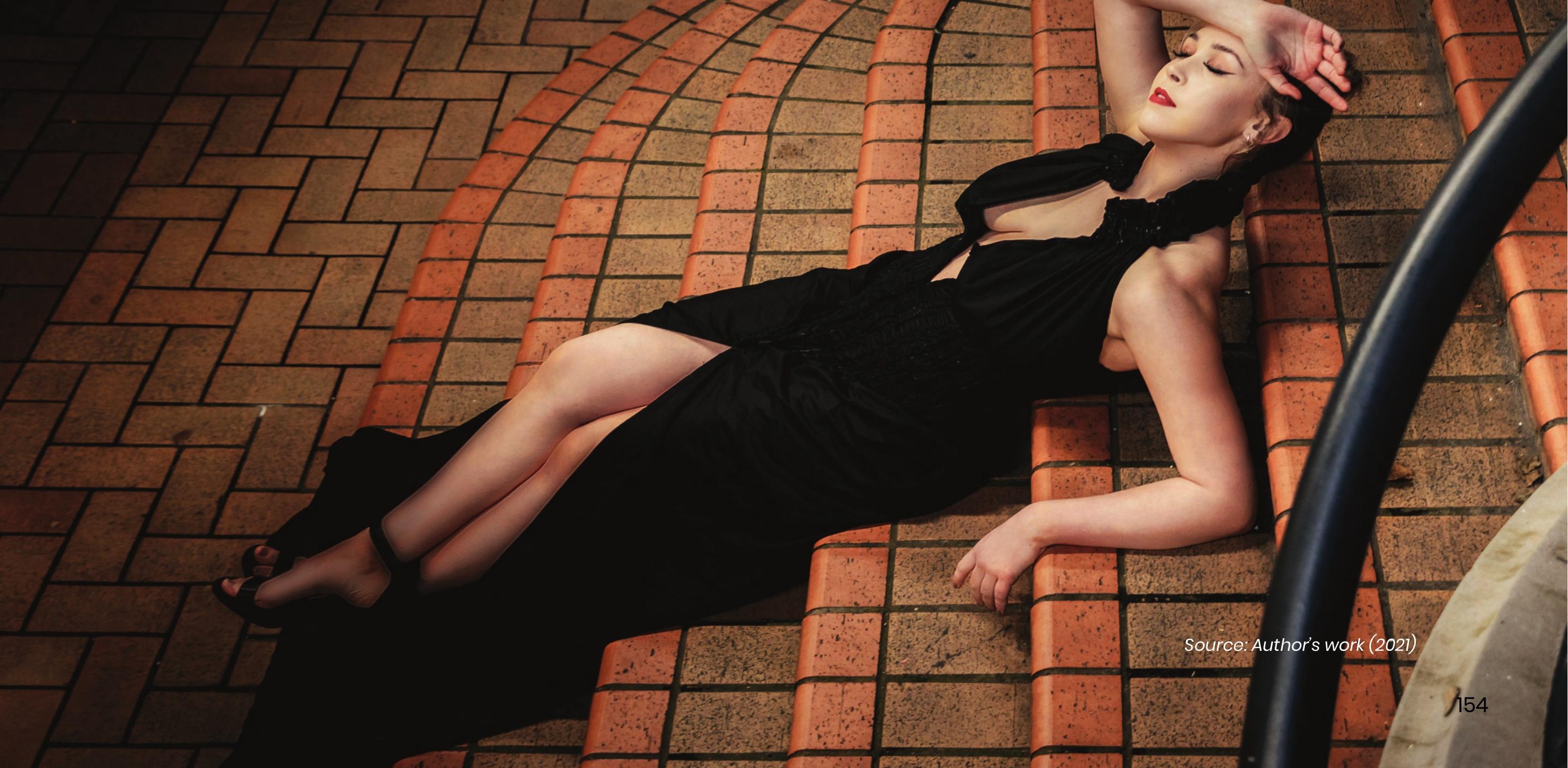


Type:

Fabric Size:

Design Characteristics:
Evening gown

Four pieces of $2500 \mathrm{~mm} \times 1400 \mathrm{~mm}$

Waistline patterns were configured close together to enhance the body form around the waist.

FIGURE 5.8A

$2 D \& 3 D$ Windows of the CLO3D Software

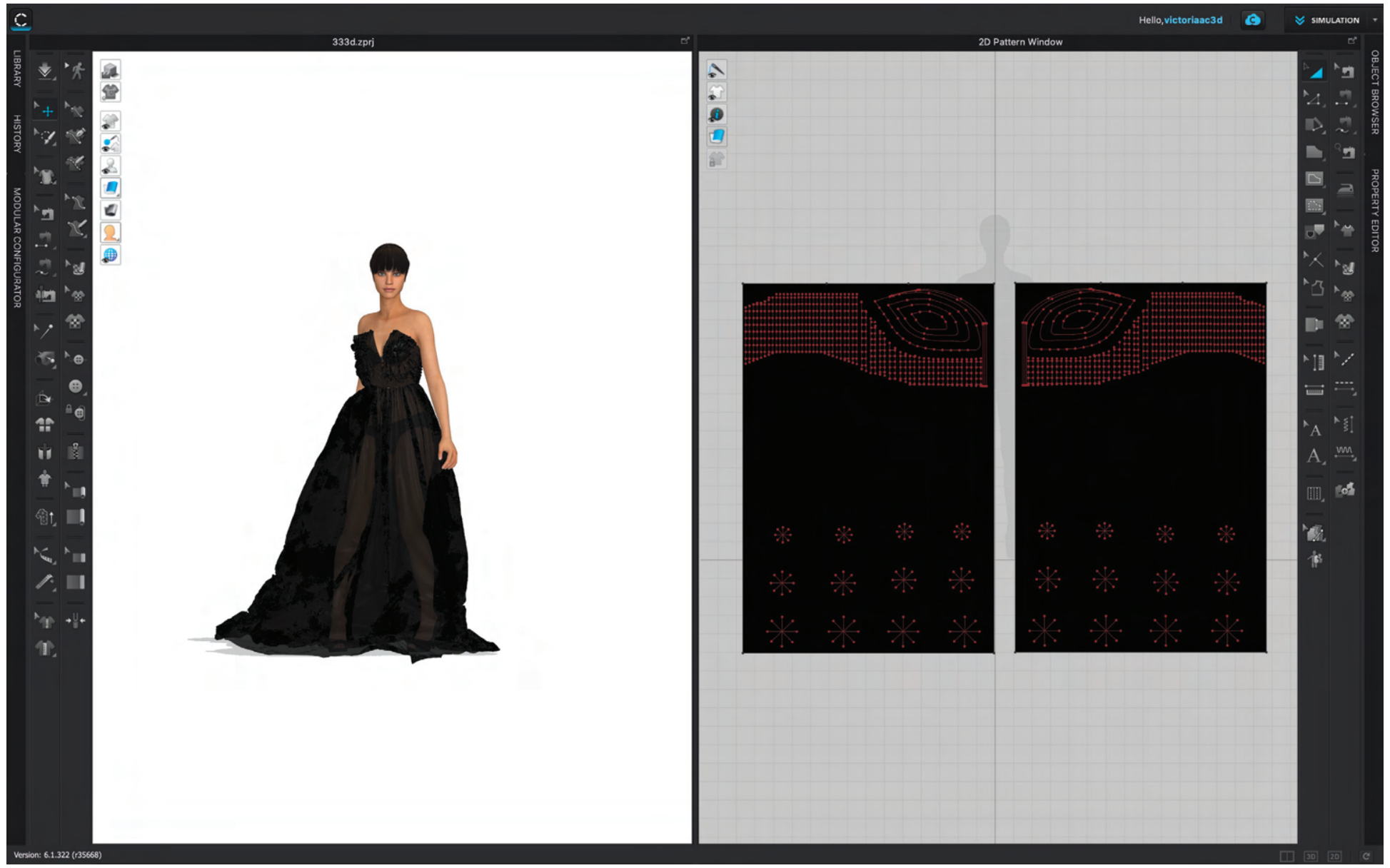

Source: Author's work (2021) 

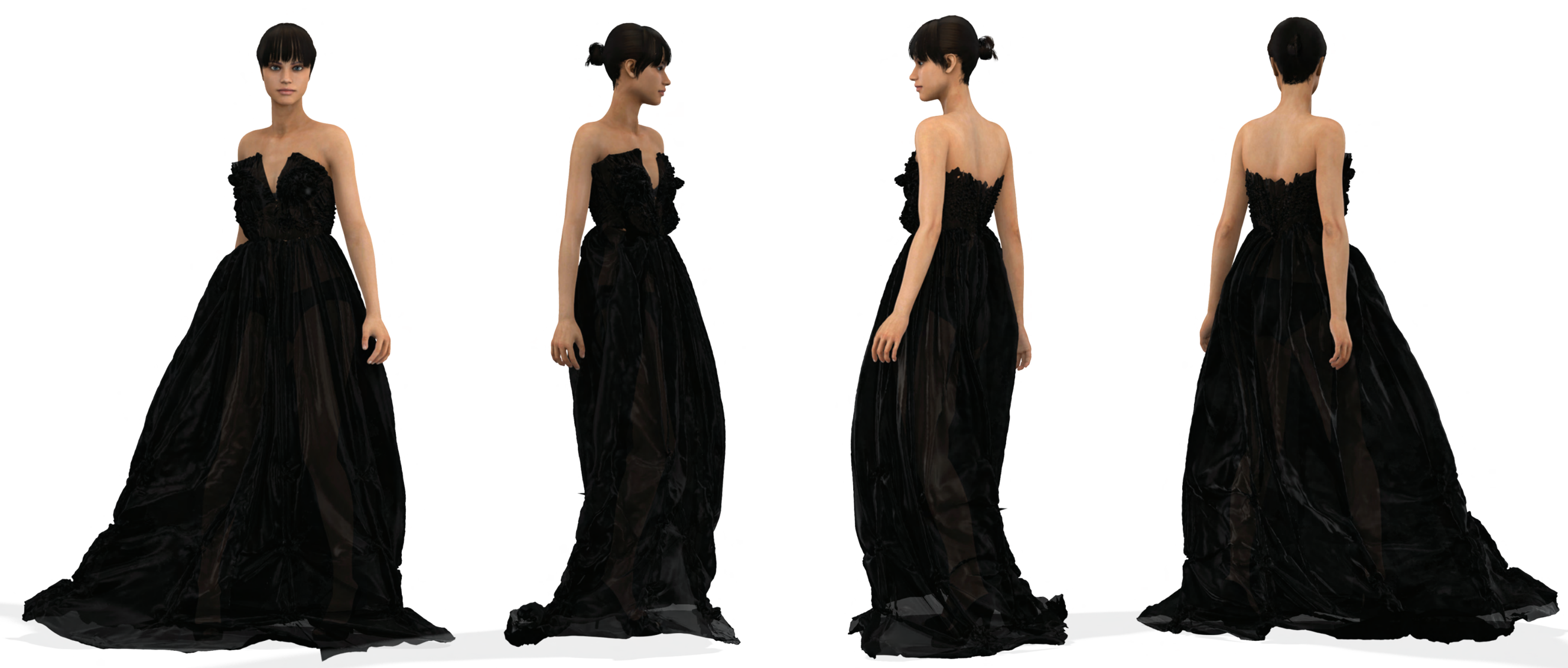
FIGURE 5.8C

Exported from CLO 3D -2D pattern into Rhino

Top F

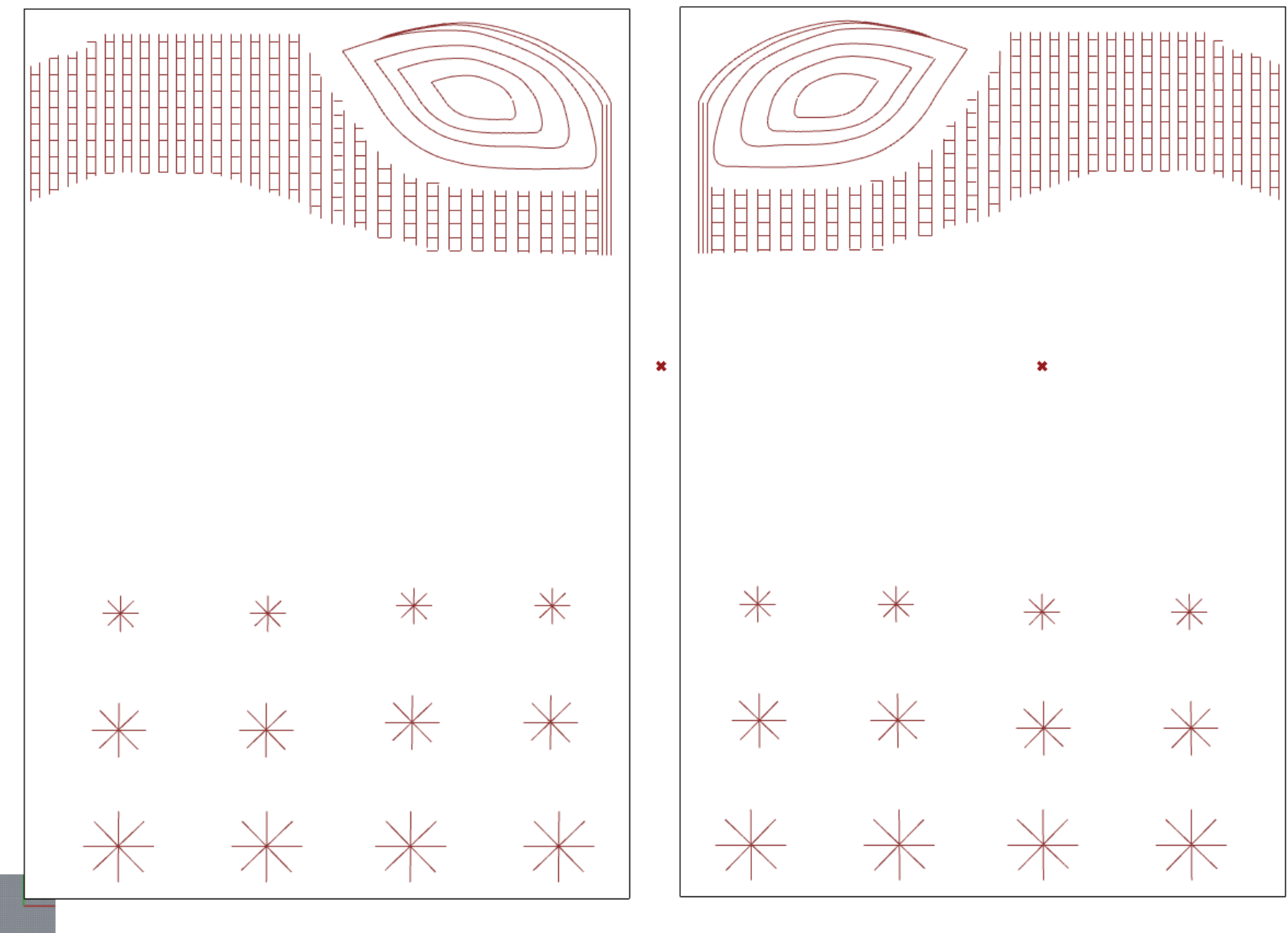

Source: Author's work (2021) 
FIGURE 5.8D

Grasshopper script

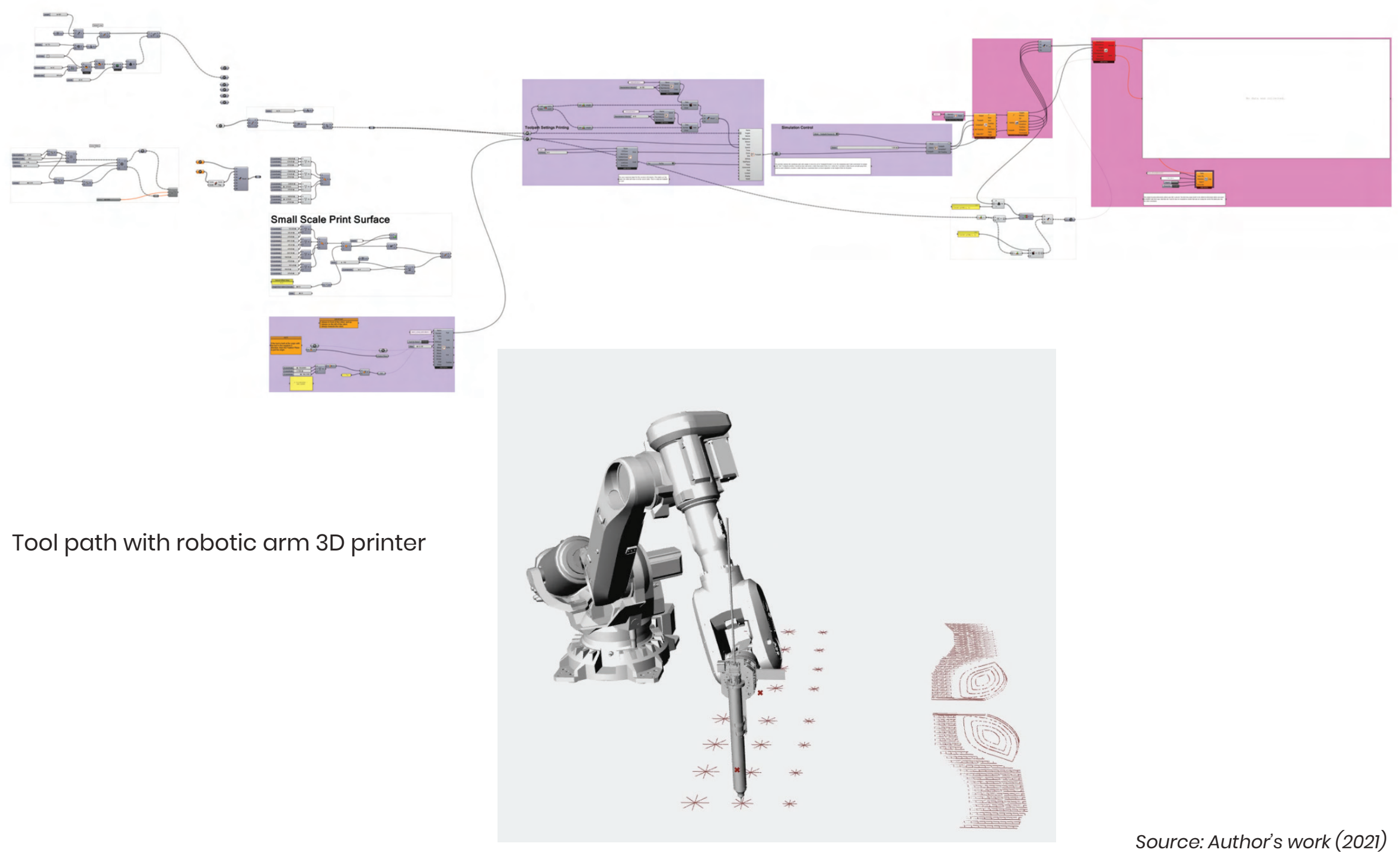



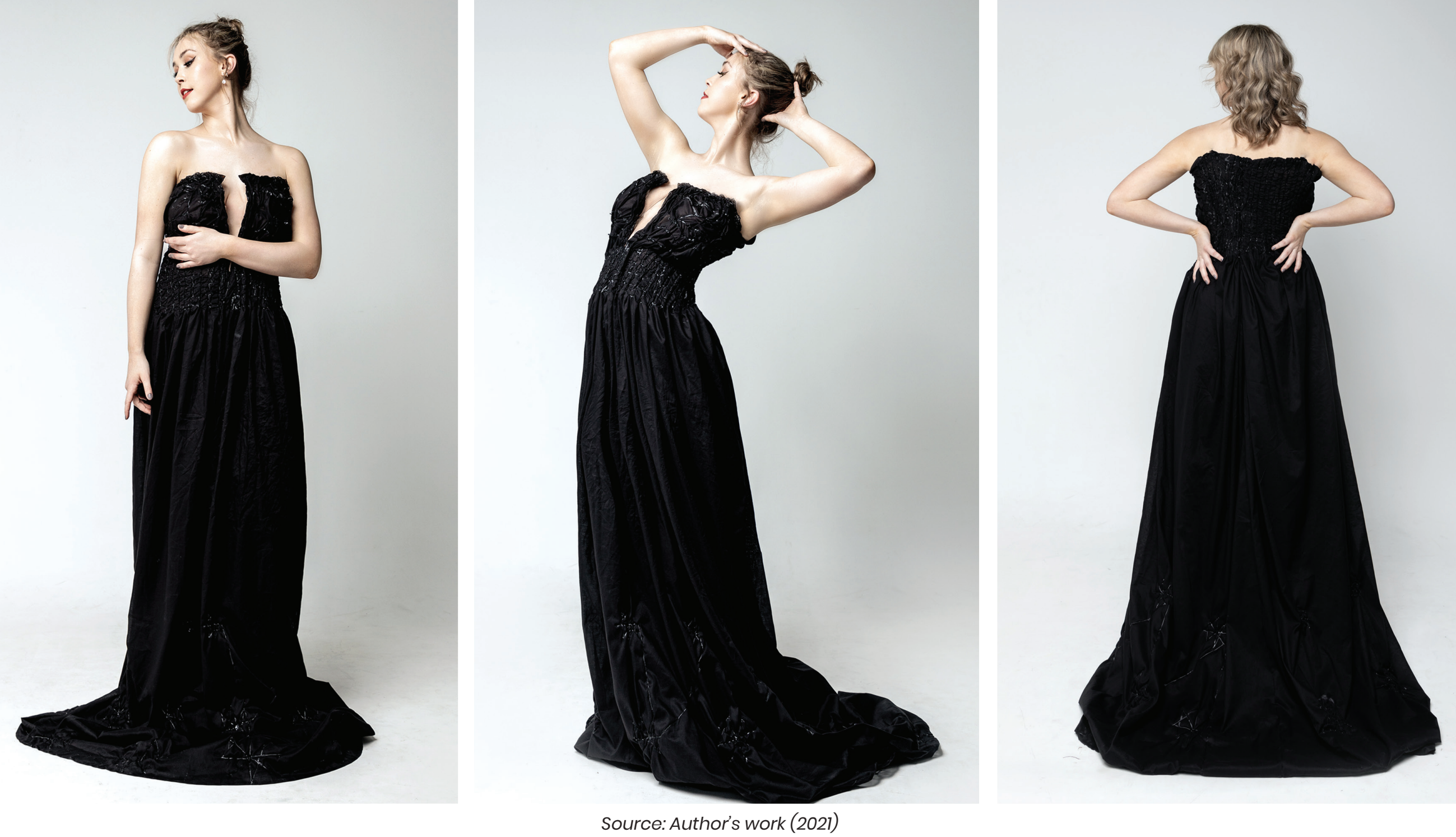


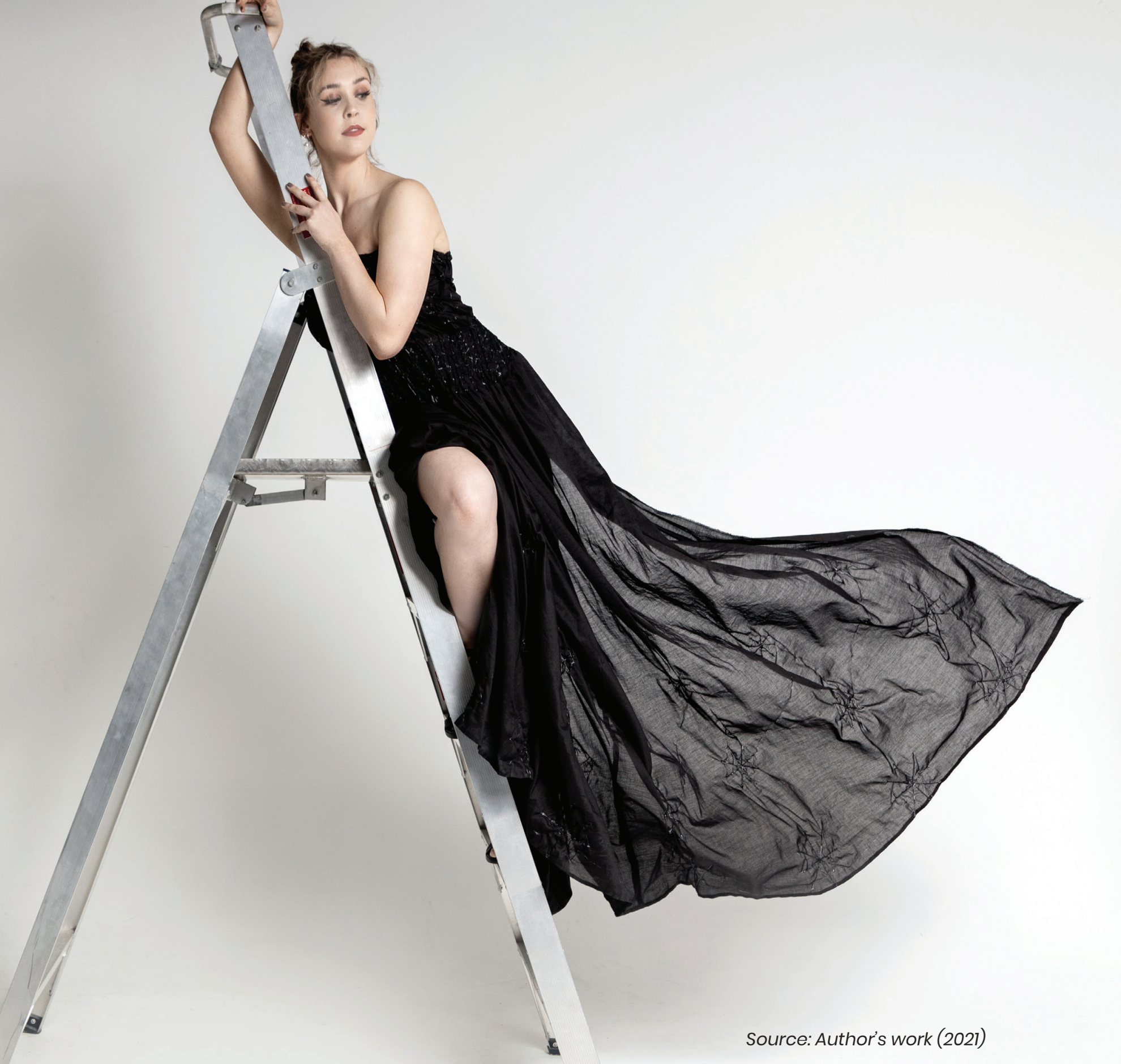






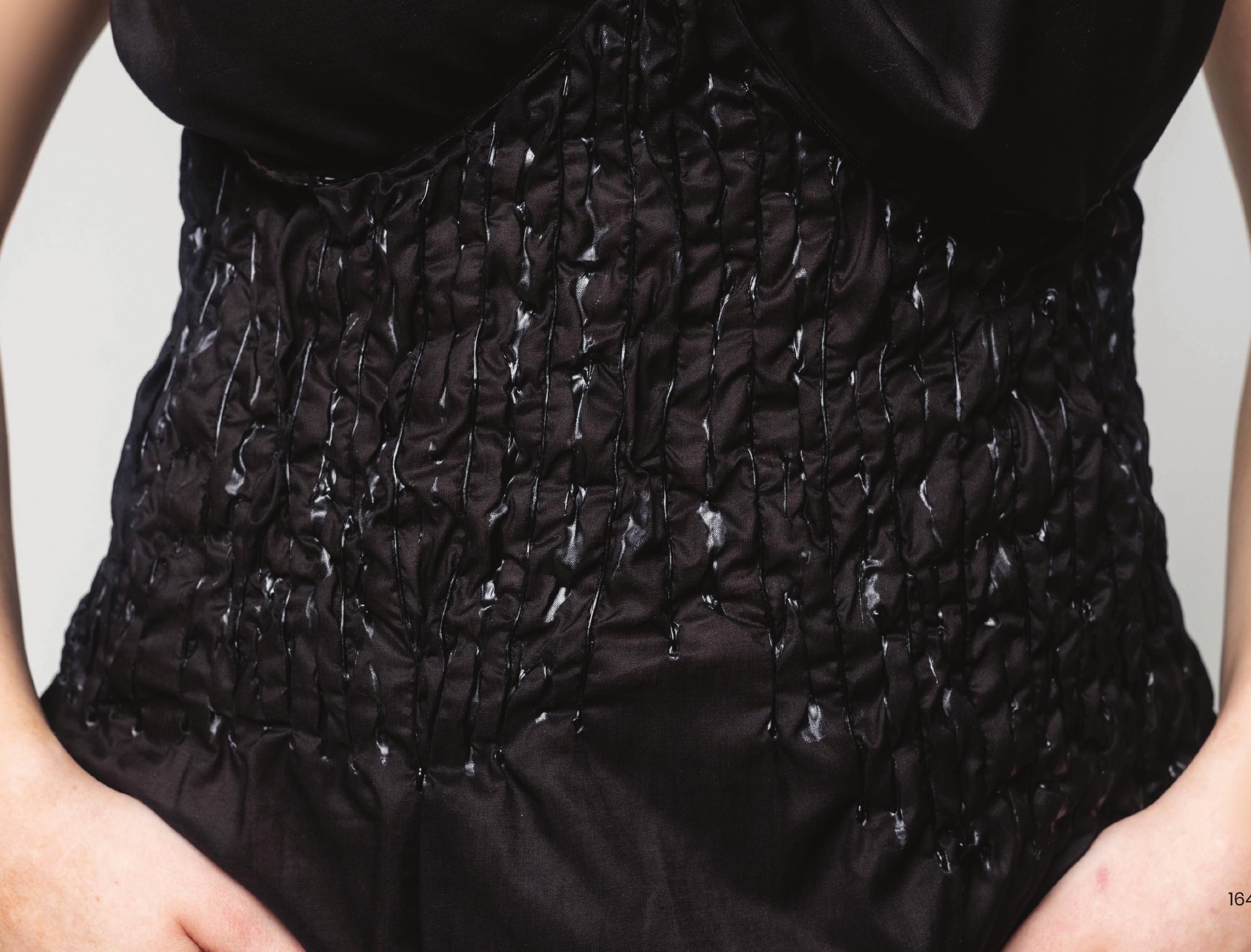




\section{DISCUSSION}

This experimental research was conducted in fulfilling the objectives of developing a sustainable bio-composite of semi-liquid nature which is suitable to be used as a 3D printing material, performing 3D prints using the developed material and adopting the outcomes into the creation of an haute couture fashion collection.

The salient inspiration for sustainable material development was the involvement of mycelium in recent precedence designs that came across during the literature survey. The author wanted to adopt the growth of mycelium into an haute couture fashion collection through 3D printing. The initial purpose of the developed semi-liquid culture was to provide a nutritional medium for the extrusion and growth of mycelium.

Through a series of experiments, the growth of mycelium and the ability to transform the growth into different mediums and substrates were developed and resulted in a number of design directions, based on the aesthetics of mushrooms. Sample areas of these designs were further trialed through automated 3-dimensional extrusion experiments on selected fabric substrates using a developed digital workflow, integrating parametric modelling of $2 \mathrm{D}$ patterns and physics simulation on virtual mannequins.

In the areas of architecture and design, according to the precedent studies, designers have used digital techniques of 3D printing by either incorporating mycelium inoculated growing mediums into 3D printed scaffolds or extruding the mycelium inoculated pastes into 3D forms in highly controlled environments. On the other hand, fashion designers have been developing non-digital processes to produce mycelium sheet material similar to leather, as sustainable alternatives. By blending the initial experimental results with inspirations from previous design and architectural projects, two systems of making MIPM and PAPM were identified and developed with extrusion trials, resulting in design opportunities in both design directions. 
However, the shrinking system using potato agar printable medium (PAPM) for the extrusion material was developed for the final collection, due to the limited laboratory conditions available for the extrusion, incubation and desiccation of the mycelium inoculated printable medium (MIPM), on a large scale.

An overriding interest in more sustainable approaches for Haute Couture Fashion guided this study with the adoption of and development of a bio-based 3D/4D printable material, selection of sustainable fabrics minimizing fabric waste and tailoring strategies within the unique digital workflow. Furthermore, by reducing the conventional physical processes of fashion garment production by integrating digital workflow elements produced a more seamless sustainable production process.

With improved access to larger equipment, one could follow the same experimental research approach and the MIPM design direction could be developed further, if a dark chamber and an industrial oven of adequate size were available.

In this research, the extrusion substrate used for the final designs was cotton fabric, which is a commercially available biodegradable material. Further, there are two areas in which this research might be extended. Firstly, in-depth exploration of the sustainable substrates and their performance in relationship to the shrinking system and secondly a material scientist could evaluate a range of extrusion formular and develop a novel bio-composite material with enhanced functionality. 


\section{IMPLICATIONS \& APPLICATIONS}

Implications from this research can be further developed through different approaches such as scientific experiments, bio-based product development, garment design techniques and trims.

From a scientific point of view, the molecular structure of the printable agar medium could be manipulated to obtain different properties. From a design perspective, the consistency of the same print medium could be varied to produce 3D forms.

The garment design technique developed here, has been influenced by the A-POC concept as it incorporates digital strategies and minimizes waste by utilization of the whole width of the fabric. By introducing different garment constructing techniques such as draping and pattern making, these 3D/4D printed bio-based haute couture collections and accessories could be expressed in various customizable forms. 


\section{SUSTAINABILITY ASSURED}

This is a challenging experimental study of blending biology and digital techniques to come up with a product that would re-define the ways of approaching high-end fashion form production.

Through the successful implementation, I have been able to establish

- A potentially new method of generating 3D garment forms

- A process to reject the excessive use of material, manpower and machinery during the conventional practices

- A product that does not end up in landfills unlike existing non-degradable haute couture wear. Once 'The Black Liner' collection reaches the end of its product life, it could just be buried or composted in a garden. 


\section{LIMITATIONS}

This design research was conducted by following the Material Driven Design Method. Hence, the level of the scientific analysis was somewhat limited. However, if more rigorous scientific analysis had taken place with the inclusion of engineering performance analysis, further advanced results could have been the potential.

Due to the limited facilities available at the university workshop and the design studio for the sterilization and desiccation processes, the dimensions of the final design forms had to be adjusted accordingly. More varieties of results could have been achieved if production could be conducted on a larger scale. Due to the lack of laboratory conditions and as growth rates the diffident species of mycelium was unknown, differential growth was experienced in some of the culture samples prepared during different experimental phases and hence the incubation upon extrusion could not be equally compared. Similarly, the likelihood of the digitally extruded samples being contaminated, was high due to the absence of humidity-controlled growing laboratory environments on a large scale. 


\section{FUTURE RESEARCH}

There are a number of areas that this research and the developed methods might influence, however the discussion here concentrates on the next steps that might be adopted in the future.

Using the methods employed in this research, further development and control of the shrinkage system to create 3D form could be conducted by developing different extrusion patterns and by exploring how this method works on a range of fabrics as well as potentially creating new 3D forms without a substrate fabric.

Mixing different colours to generate unique patterns could also be another area where this research could be extended and thus, offering further aesthetic opportunities.

The edge treatment of the fabric and the integration of fixings seamlessly into the digital making system has been identified as areas where further research could be conducted with possibilities to replicate decorative elements such as sequins and beads.

When the pigmented printed patterns using mycelium inoculated agar medium are desiccated, they tend to lose their colours. Therefore, it could be experimented how developments could be made using different natural dying techniques to retain the colour forms.

Through varying the composition of the printable agar medium, it could be investigated whether improved shrinkage ratios could also be achieved.

The overriding theme of sustainability through these experiments in the reduction of waste using nature and natural processes controlled through digitalization, might act as a springboard for further research. If this methodology gains international exposure, the theories behind waste reduction in this study might get adopted by other designers or collaboration might take place and thus illustrating the reduction of the excesses waste in the haute couture fashion industry. 


\section{CONCLUSION}

The experiments and the developing of a digital system using a bio-composite material to create 3D/4D printed garment forms open up and illustrate opportunities for the future of sustainable fashion.

Adopting a cross-disciplinary approach of Industrial Design and Fashion Design through the merging of 3D rendering software, parametric design processes and robotic production, has taken this experimental design method into novel dimensions within the haute couture fashion industry.

Traditional haute couture techniques invest considerable amount of time and effort in labour intensive hand techniques in the areas of, pattern making, tailoring and fitting the garments to body forms. The methods developed in this research illustrates a parametric digital workflow that combines elements of natural shrinkage to create digitally fitted garments. The developed software system enabled the precise digital placement of biomaterial through robotic printing, enabling the development of a range of patterns that has produced a series of 3D forms.

The direction in which this research has taken, is not the position intended at the beginning of the research, especially with regards to the use of mycelium in the final design stages. However, the uniqueness of the design direction, incorporating the natural shrinkage of printable agar, which led to the generation of the collection of tailored haute couture fashion garments, is one that I am proud of. 


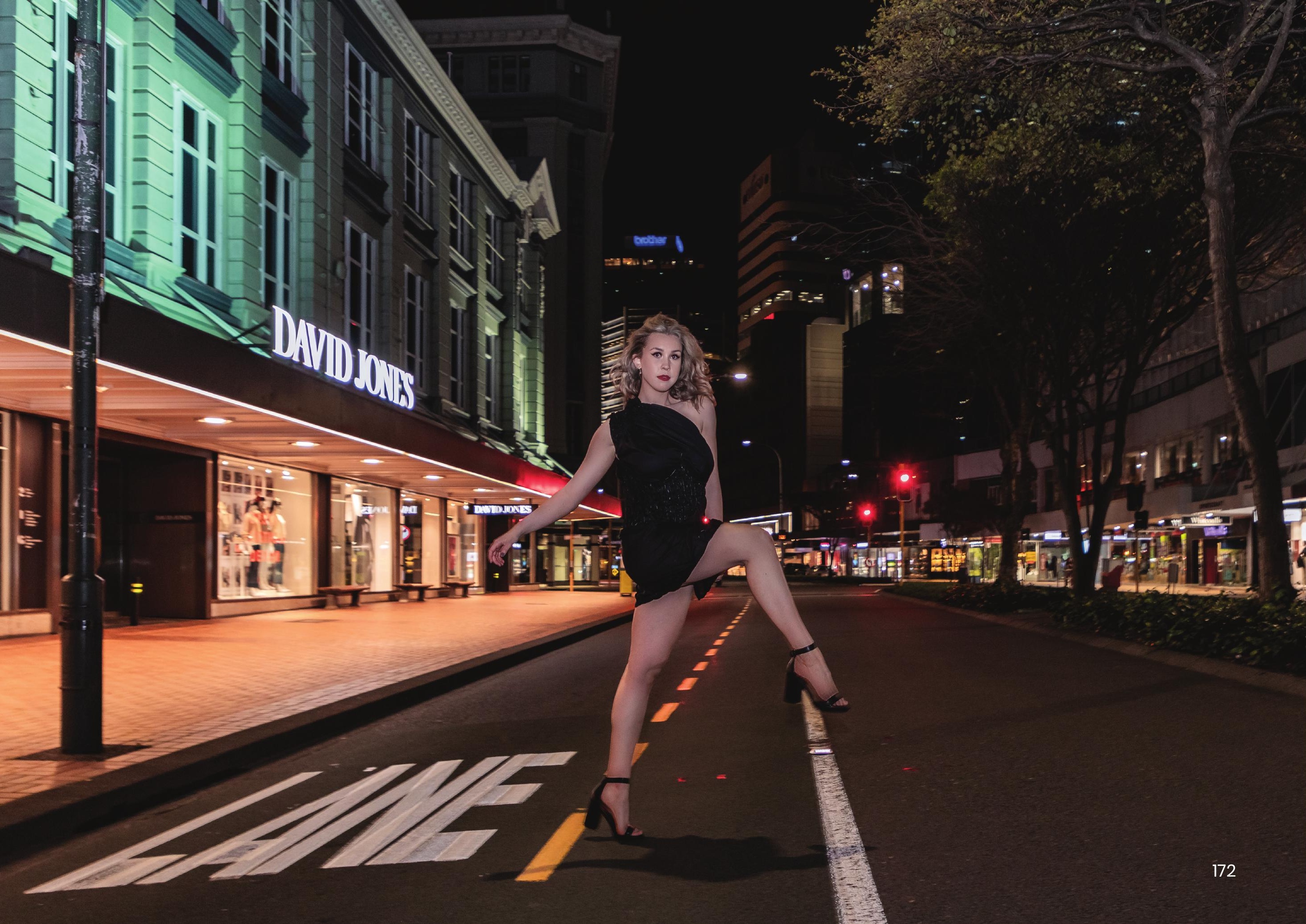




\section{REFERENCES}

Alexandrea, P. (2020, July 10). 3D Printed Fashion: Top 13 3D Printed Fashion Designs. Retrieved from 3Dnatives: https://www.3dnatives.com/en/3d-printing-fashion-designs150620174/\#!

Avinc, O., Yildirim, F., Yavas, A., \& Kalayci, E. (2017). 3D Printing Technology and its Influences on the Textile Industry. Int. J. Ind. Electron. Electr. Eng., 5, 37-43.

Beecroft, M. (2016). 3D printing of weft knitted textile based structures by selective laser sintering of nylon powder. IOP Conf. Ser. Mater. Sci. Eng.(012017), 137.

Beecroft, M. (2019). Digital interlooping: 3D printing of weft-knitted textile-based tubular structures using selective laser sintering of nylon powder. Int. J. Fash. Des. Technol. Educ., 12, 218-224.

Bergmann, C., \& Stumpf, A. (2013). Biomaterials. In: Dental Ceramics. Topics in Mining, Metallurgy and Materials Engineering. Berlin, Heidelberg: Springer, https://doi.org/10.1007/978-3-642-38224-6_2.

Bingham, G., \& Hague, R. (2013). Efficient three dimensional modelling of additive manufactured textiles. Rapid Prototyp. J., 19, 269-281.

Cho, R. (2017, December 13). The Truth About Bioplastics. Retrieved from Colombia University: https://blogs.ei.columbia.edu/2017/12/13/the-truth-about-bioplastics/

Choi, J., Kwon, C., Jo, W., Lee, H., \& Moon, M. (2015). 4D Printing Technology: A Review. 3D Printing and additive manufacturing, 2(4), 159-167.

Collet, C. (2016). Self-patterned Mycelium Rubber and Mycelium Lace. 
Collet, C. (2017, June 19). Grow-Made Textiles. Retrieved from Alive, Active, Adaptive, EKSIG International Conference on Experiential Knowledge and Emerging Materials, The New Institut, Rotterdam, The Netherlands:

http://www.eksig2017.com/about-eksig/

Ducheyne, P. (2017). Comprehensive Biomaterials II (2nd ed.). Elsevier Ltd.

Ecovative Design. (2020). We Grow Materials. Retrieved from Ecovative Design: https://ecovativedesign.com/ european-bioplastics. (2020). european-bioplastics. Retrieved from What are bioplastics?:

european-bioplastics.org/bioplastics/

Farahani, R., Dube, M., $\delta$ Therriault, D. (2016). Three dimensional printing of multifunctional nanocomposites: manufacturing techniques and applications. Adv Mater, 28(28), 5794-5821.

Frearson, A. (2014, July 01). Tower of "grown" bio-bricks by The Living opens at MoMA PS1. Retrieved from Dezeen: https://www.dezeen.com/2014/07/01/tower-of-grown-bio-bricks-by-the-living-opens-at-moma-psl-gallery/

Ge, Q., Sakhaei, A., Lee, H., Dunn, C., Fang, N., \& Dunn, M. (2016). Multimaterial 4D printing with tailoring shape memory polymers. Sci Rep, 6(31110).

Gibson, L. (2018, November 28). Fabric Friday: petroleum-based manufactured fibers. Retrieved from Oliver + S: https://oliverands.com/community/blog/2013/05/fabric-friday-petroleum-based-manufactured-fibers.html

Gladman, S., Matsumoto, E., Nuzzo, R., Mahadevan, L., \& Lewis, J. (2016). Biomimetic 4D printing. Nat. Mater, 15(4), 413-418.

Grain, E. (2019). Textiles, Identity and Innovation: Design the Future. In G. Montagna, \& C. Carvalho (Eds.), Surface, Digital and Virtual Textiles-An Analysis of 3D Printed Textile Structures. London, UK: Taylor \& Francis Group. 
Grown bio. (2020). Retrieved from Grown bio: https://www.grown.bio/

Hahn, J. (2021, March 24). Stella McCartney creates clothes from mycelium leather to foster a "kinder fashion industry".

Retrieved from Dezeen: https://www.dezeen.com/2021/03/24/mylo-stella-mccartney-mycelium-leather-fashion/

Hudecki, A., Kiryczyński, G., \& Łos, M. (2019). Chapter 7 - Biomaterials, Definition, Overview. In A. Hudecki, G. Kiryczyński, \& M. J. Łos, Stem Cells and Biomaterials for Regenerative Medicine (pp. 85-98). Elsevier Inc.,

https://doi.org/10.1016/B978-0-12-812258-7.00007-1.

Jacquemyn, H., Waud, M., Brys, R., Lallemand, F., Courty, P.-E., Robionek, A., $\delta$ Selosse, M.-A. (2017). Mycorrhizal Associations and Trophic Modes in Coexisting Orchids: An Ecological Continuum between Auto- and Mixotrophy. Front. Plant Sci. , 8:1497, doi: 10.3389/fpls.2017.01497.

Joshi, S., Rawat, K., Karunakaran, C., Rajamohan, V., Mathew, A., Koziol, K., .. Balan, A. (2019). 4D printing of materials for the future: Opportunities and challenges. Appl. Mater. Today, 100490.

Karana, E., Blauwhoff, D., Hultink, E.-J., \& Camere, S. (2018). When the Material Grows: A Case Study on Designing (with) Mycelium-based Materials. International Journal of Design.

Khan, I., Siwe, L., Yuan, L., Wanheng, L., \& Anthony, S. (2015, April 13). 4D printing with smart materials," Slideshare. Retrieved October 20, 2020, from https://www.slideshare.net/Funk98/4d-printing-with-smart-materials

Khurshid, Z., Najeeb, S., \& Zafar, S. F. (2019). Advanced Dental Biomaterials. Elsevier Ltd.

Koetsier, E. (2020). Designing with Mycelium. Retrieved from www.elizekoetsier.com: https://www.elizekoetsier.com/projects/thesis-designingwithmycelium 
Krassenstein, B. (2014, June 26). 3D Printing With Fungus-Artist Creates Chairs and Other Objects Out of Mushrooms. . Retrieved from 3DPrint.Coml The Voice of 3D Printing / Additive Manufacturing:

https://3dprint.com/7279/3d-print-fungus-mycelium/

Lendlein, A. (2010). Progress in activelt moving polymers. Journal of Materials Chemistry, 20, 1-5.

Liu, Y., Genzer, J., \& Dickey, M. (2016). 2D or no 2D": Shape programming polymer sheets. Progress in polymer science, 52, 79-106.

Meng, H., Li, H., \& Ibekwe, S. (2012). A review of stimuli responsive polymers for smart textile applications. Smart Mater Struct, $21(1)$.

MIT Media Lab People. (2020). MIT Media Lab People. Retrieved from Neri Oxmen: Projects:

https://www.media.mit.edu/people/neri/projects/

Molloy, I. (2017). Designed Deposition: Freeform 3D Printing for Digitally Crafted Artefacts. Wellington: Victoria University of Wellington, School of Design.

Momeni, F., Liu, X., \& Ni, J. (2017). A review of 4D printing. Mater. Des., 122, 42-79.

Mu, X., Bertron, T., Dunn, C., Qiao, H., Wu, J., \& Zha, Z. (2017). Porous polymeric materials by 3D printing of photocurable resin. Mater Horiz, 3, 442-449.

Nadgorny, M., Xiao, Z., Chen, C., \& Connal, L. (2016). Three dimensional printing of ph-responsive and functional polymers on an affordable desktop printer. ACS Appl Mater Interfaces, 8(42), 28946-28954. 
Ngo, T.-D. (2017). Natural Fibers for Sustainable Bio-Composites. Natural and Artificial Fiber-Reinforced Composites as Renewable Sources.

Nkomo, N. Z. (2018). A Review of 4D Printing Technology and Future Trends. Vanderbijlpark, South Africa: Eleventh South African Conference on Computational and Applied Mechanics.

Nutrient Cycles. (n.d.). Retrieved from Lumen Learning:

https://courses.lumenlearning.com/trident-boundless-microbiology/chapter/nutrient-cycles/

Oxman. (2020). Aguahoja. Retrieved from Oxman: https://oxman.com/projects/aguahoja

Pei, E. (2014). 4D Printing:dawn of an emerging technology cycle. Assembly automation, 34(4), 310-314.

Pei, E., Shen, J., \& Watlin, J. (2015). Direct 3D printing of polymers onto textiles: Experimental studies and applications. Rapid prototyping journal, 21(5), 556-571.

Perry, A. (2017). 3D-printed apparel and 3D-printer: Exploring advantages, concerns, and purchases. Int. J. Fash. Des. Technol. Educ. , 11, 95-103.

Porter, P. (2017, September 15). THE 3D PRINTING S PANORAMA IN FASHION DESIGN. Retrieved from SILO.TIPS: https://silo.tips/download/the-3d-printing-s-panorama-in-fashion-design

Ramesh, S., Reddy, S., Usha, C., Niranjan Kumar, N., Adithyakumar, C., \& Reddy, M. (2018). Advancements in The Research of 4D Printing-A Review. IOP Conf. Series: Materials Science and Engineering, 376(2018), 1-11. 
Rehman, S., Ali, M., Zuber, M., Zia, K., \& Iqbal, R. (2017). Chapter 20 - Future Prospects of Algae-Based Materials. In S. Rehman, M. Ali, M. Zuber, K. M. Zia, \& R. Iqbal, Algae Based Polymers, Blends, and Composites: Algae Based Polymers, Blends, and Composites (pp. 687-691). Elsevier Inc.

Reisky, L., Préchoux, A., Zühlke, M., Bäumgen, M., Robb, C., Gerlach, N., .. Hehemann, J. (2019). A marine bacterial enzymatic cascade degrades the algal polysaccharide ulvan. Nature Chemical Biology.

Rosenau, J., \& Wilson, D. (2014). Apparel Merchandising: The Line Starts Here. New York, NY, USA: Fairchild Books.

Sayner, A. (2021, February 28). How To Grow Mushrooms: The Ultimate Guide. Retrieved from GroCycle: https://grocycle.com/how-to-grow-mushrooms/

Schmelzeisen, D., Koch, H., Pastore, C., \& Gries, T. (2018). Narrow and Smart Textiles . In Y. Kyosev, B. Mahltig, \& A. Schwarz-Pfeiffer (Eds.), 4D Textiles: Hybrid Textile Structures that Can Change Structural Form with Time by 3D Printing. Cham, Switzerland: Springer International Publishing.

Sen, A. (2015, June). Biomaterials in textiles: Poly-lactic acid fibres. Retrieved from https://www.fibre2fashion.com/: https://www.fibre2fashion.com/industry-article/7574/biomaterials-in-textiles-poly-lactic-acid-fibres

Sen, A. (2015, June). Biomaterials in textiles: Poly-lactic acid fibres. Retrieved from fibre2fashion.com: https://www.fibre2fashion.com/industry-article/7574/biomaterials-in-textiles-poly-lactic-acid-fibres

Shin, D., Kim, T., \& Kim, D. (2017). Review of 4D printing materials and their properties. International journal of precision engineering and manufacturing green technolgy, 4(3), 340-357.

Stanton, M., Trichet-Paredes, C., \& Sanchez, S. (2015). Applications of three-dimensional (3D) printing for microswimmers and bio-hybrid robotics. Lab on a chip, 15(7), 1634-1637. 
Sundakar, K. H. (2012, April). Bio-composites. Retrieved from

https://www.fibre2fashion.com/industry-article/6185/bio-composites

Sundaram, S., Kim, D., Baldo, M., Hayward, R., $\delta$ Matusik, W. (2017). 3D printed self folding electronics. ACS Appl Mater Interfaces, 9(37), 32290-32298.

Thomas, S., Grohen, Y., \& Ninan, N. (2015). Nanotechnology Applications for Tissue Engineering. Elsevier Inc.

Tibbits, S. (2014). 4D Printing: Multi material shape change. Architectural design, 84, 116-121.

Uysal, R., $\delta$ Stubbs, J. (2019). A New Method of Printing Multi-Material Textiles by Fused Deposition Modelling (FDM). Tekstilec, $62,248-257$.

Valtas, A., \& Sun, D. (2016). 3D Printing for Garments Production: An Exploratory Study. J. Fash. Technol. Text. Eng., 4.

What is a composite material? A definitive guide. (n.d.). Retrieved from

https://www.twi-global.com/technical-knowledge/faqs/what-is-a-composite-material

What is SAP - superabsorbent polymers. (2019). Retrieved from Edana:

https://www.edana.org/nw-related-industry/what-is-sap

Yap, Y., \& Yeong, W. (2014). Additive manufacture of fashion and jewellery products: A mini review. Virtual Phys. Prototyp., 9, 195-201.

Yap, Y., \& Yeong, W. (2014). Additive manufacturing of fashion and jewellery products: A mini review: This paper provides an insight into the future of 3D printing industries for fashion and jewellery products. Virtual and physical prototyping, 9 (3),

195-201. 
Zapfel, D. (2019). How 3D Printing in the Textile Industry Is Leading Into a New Era; . Wien, Austria,: Lead Innov. Manag.

Frankel, L., $\delta$ Racine, M. (2010). The Complex Field of Research: for Design, through Design, and about Design.

Hapuarachchi, K. K. (2018). High diversity of Ganoderma and Amauroderma (Ganodermataceae, Polyporales) in Hainan Island, China. Mycosphere, 9(5), 931-982. https://doi.org/10.5943/mycosphere/9/5/1

Kubala, J. (2018, November 6). 5 Immune-Boosting Benefits of Turkey Tail Mushroom. Healthline.

https://www.healthline.com/nutrition/turkey-tail-mushroom.

Rajasekar, S., Philominathan, P., \& Chinnathambi, V. (2013). Research Methodology.

Saunders, M. (2003). Research methods for business students. Pearson Education India.

Schumann, G. L. (2002, January 16). Easy Demonstration of Fungal Mycelium. Easy

Demonstration of Fungal Mycelium.

https://www.apsnet.org/edcenter/foreducators/TeachingNotes/Pages/EasyDemonstrationOfFungalMycelium.aspx.

Segedin, B.P.; Buchanan, P.K.; Wilkie, J.P. (1995). "Studies in the agaricales of New Zealand: New species, new records and renamed species of Pleurotus (Pleurotaceae)". Australian Systematic Botany. 8 (3): 453-482.

Sileyew, K. J. (2019). Research Design and Methodology.

Stappers, P. J., \& Giaccardi , E. (n.d.). Research through Design. The Interaction Design Foundation. https://www.interaction-design.org/literature/book/the-encyclopedia-of-human-computer-interaction-2nd-ed/researchthrough-design. 
Valverde, M. E., Hernández-Pérez, T., \& Paredes-López, O. (2015). Edible Mushrooms:

Improving Human Health and Promoting Quality Life. International Journal of Microbiology, 2015, 1-14.

https://doi.org/10.1155/2015/376387

Wasser, S. P. (n.d.). Flammulin Purified from the Fruit Bodies of Flammulina velutipes (Curt.:Fr.) P.Karst. International Journal of Medicinal Mushrooms. https://doi.org/10.1615/IntJMedMushrooms.vl.11.70

[StevenBigShroom]. (2020). Uncle Ben Tek - A Full Grow Report [Online forum post]. Reddit.

https://www.reddit.com/r/unclebens/comments/g85axs/uncle_ben_tek_a_full_grow_report/ 


\section{LIST OF FIGURES}

Figure 1.1 Classification of Bio-composites Based on Properties of Fibrous Material 4

Figure 1.2 Poly Lactic Acid Fibre 5

Figure 1.3 3D Printed Objects Using Dried Algae-Based Biomaterials by Eric Klarenbeek and Maartje Dros 7

Figure 1.4 Structure of Fungus 8

Figure 1.5 'Hy-Fi' by The Living 9

Figure 1.6 Grown Products 10

Figure 1.7 Neri Oxman's Aguahoja Project $\quad 11$

Figure 1.8 Neri Oxman's Aguahoja Collection 12

Figure 1.9 Mycelium Chair by Eric Klarenbeek 13

Figure 1.10 Experiments by Elize Koetsier, Growing Mycelium in 3D Printed Scaffolding 14

Figure 1.11 Self-patterning Mycelium Rubber 15

Figure 1.12 Tartan Mycelium, Mycelium Lace and Mycelium Velvet 16

Figure 1.13 Key Concept of Blast Studio 17

Figure 1.14 Different Objects of Blast Studio Experiments 17

Figure 1.15 4DP examples that show the single strand evolving of initials "MIT" into "SAL" 21

Figure 1.16 Stratasys Object Connex Multi-material 3D Printer 22

Figure 1.17 A Stereolithography Dress from 'Hybrid Holism' Runway Collection 27

Figure 1.18 Cape and Skirt for 'Voltage' Collection by Iris van Herpen 27

Figure 1.19 Ruth E Carter and 3D STOLA 28

Figure 1.20 Setae Jacket by Julia Koerner 29

Figure 1.21 Ganit Goldstein's 'WeAreAble' Kimono Collection 30

Figure 1.22 The Two-Piece Outfit by Stella McCartney's Brand MYLO Consists of a Black Bustier and Matching Trousers 32

Figure 1.23 Kristel Peters' Growing Shoes 33

Figure 1.24 Products Made of 'Seamless' MycoTEX $\mathbb{R}$

Figure 1.25 Material Development Experiments for MycoTEX® 34 
Figure 1.26 The Bio-boomer Jacket

Figure 1.27 The Adorn Couture Dress Made with Algae Sequins

Figure 3.4 Culture Samples of Native Fungi Received from ICPM New Zealand 49

Figure 3.5 Liquid Culture prepared Using ICPM 115546

Figure 3.6 Mycelium Growth in Liquid Culture 56

Figure 3.7 Mycelium Growth in Potato Starch Agar 57

Figure 3.8 Mycelium Growth Under "Uncle Ben's Tek" Technique 59

Figure 3.9 Growth of mycelium in plastic and fibrous mediums soaked in liquid culture 60

Figure 3.10 Growth of Mycelium on Fabric Substrates Soaked in PDA 64

Figure 3.11 Growth of Mycelium from Coffee-Wood Straw Medium, Upon Extrusion onto a Plastic Wireframe 67

Figure 3.12 Experimental Procedure of Mycelium Growth in Extruded Tissue Paper Pulp and PVA Medium 69

Figure 3.13 Free Growth of Mycelium in PDA Medium and Through Cotton Yarn 71

Figure 3.14 Workflow Development of the Experimental Designs Using the Syringe Printing Technique 72

Figure 3.15 Preparation of the Semi-Liquid culture Medium 74

Figure 3.16 Inoculation and Extrusion Process of the Semi-Liquid Culture 75

Figure 3.17 Outcomes Within Two Weeks of the Extrusion of Semi-Liquid Culture With Mycelium 76

Figure 3.18 Behaviour of the semi-liquid culture on fabric and paper surfaces 77

Figure 3.19 Mycelium Growth on Fabric Surfaces Using the Extruded Semi-Liquid Culture 80

Figure 3.20 The laboratory drying oven used for desiccation 82

Figure 3.21 Baked products using grown mycelium on fabric surfaces soaked in PDA 83

Figure 3.22 Baked products using grown mycelium through the extruded semi-liquid culture on fabric surfaces 84

Figure 4.1 ABB's IRB 6700 robotic arm printer 86 
Figure 4.2 The $4000 \mathrm{ml}$ extruder used in the robotic arm printer

Figure 4.3 Filling the Extruder with Printable Agar Medium

Figure 4.4 Fixing the $3 \mathrm{~mm}$ Diameter Nozzle Head to the Extruder

Figure 4.5 Fixing the Extruder to Axis 6 of the Robotic Arm Printer and Tightening it Using the Velcro Straps 88

Figure 4.6 Fixing the Controller Circuit Module into the Robotic Arm Printer

Figure 4.7 The Ready-To-Use Extruder Fixed Robotic Arm Printer with the FlexPendant interaction Panel

Figure 4.8 Preparation of the Red-Coloured Printable Agar Medium

Figure 4.9 Workflow diagram of digital extrusion exercises conducted using the printable agar medium on paper and fabric surfaces

Figure 4.10 Extrusion Outcomes During the Test Run to Adjust the Speed of the Robotic Arm 97

Figure 4.11A Extrusion Pattern

Figure 4.11B Developed Grasshopper Script

Figure 4.11c Observations of the First Pattern on Different Substrates Upon Extrusion

Figure 4.12a Extrusion Pattern

Figure 4.12b Developed Grasshopper Script

Figure 4.13c Observations of the Third Pattern on Different Substrates Upon Extrusion 103

Figure 4.14a Extrusion Pattern 104

Figure 4.14b Developed Grasshopper Script 104

Figure 4.14c Observations of the Fourth Pattern on Different Substrates Upon Extrusion 104

Figure 4.15a Extrusion Pattern 105

Figure 4.15b Developed Grasshopper Script 105

Figure 4.15c Observations of the Fifth Pattern on Different Substrates Upon Extrusion 105

Figure 4.16 Workflow Diagram of Digital Extrusion Exercises Conducted Using the Mycelium Inoculated Printable Agar 
Figure 4.17 Growth of Mycelium in 3D Printed Patterns on Fabric Substrates, Seven Days After Extrusion 
